\title{
Enantioselective 1,2-Alkylhydroxylmethylation of Alkynes via Chromium/Cobalt Cocatalysis
}

\section{SUPPORTING INFORMATION}

Hanwen Zhang, Bin Chen, Guozhu Zhang*

State Key Laboratory of Organometallic Chemistry, Shanghai Institute of Organic Chemistry, Center for Excellence in Molecular Synthesis, University of Chinese Academy of Sciences, Chinese Academy of Sciences, 345 Lingling Road, Shanghai 200032, P. R. China.

(*Email: guozhuzhang@sioc.ac.cn)

\section{CONTENTS}

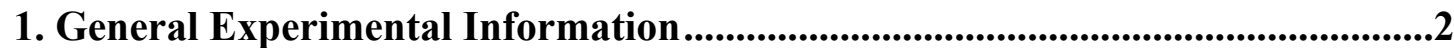

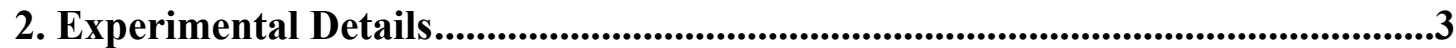

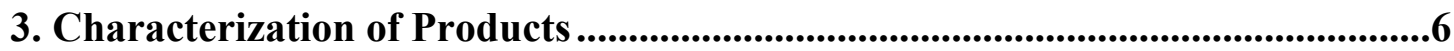

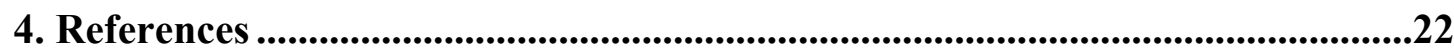

5. Copies of NMR spectra, HPLC Chromatographs and NOESY Spectra ...........23 


\section{General Information}

NMR spectra were recorded at room temperature on the following spectrometers: Agilent (400 MHz) and VARIAN (400 MHz). Chemical shifts are given in ppm and coupling constants in $\mathrm{Hz}$. $1 \mathrm{H}$ spectra were calibrated in relation to the reference measurement of TMS $(0.00 \mathrm{ppm}) .{ }^{13} \mathrm{C}$ spectra were calibrated in relation to deuterated solvents, namely $\mathrm{CDCl}_{3}(77.16 \mathrm{ppm})$. The following abbreviations were used for $1 \mathrm{H}$ NMR spectra to indicate the signal multiplicity: $s$ (singlet), $d$ (doublet), $t$ (triplet), $q$ (quartet) and $\mathrm{m}$ (multiplet) as well as combinations of them. When combinations of multiplicities are given the first character noted refers to the largest coupling constant. High performance liquid chromatography (HPLC) was carried out with Agilent 1260 Infinity on a UV spectrophotometric detector (Agilent). For ESI+-spectra an EI-HR (GC-TOF) spectrometer was applied. Infrared Spectroscopy (IR) was processed on an FT-IR spectrometer named Nicolet 380. The method is denoted in brackets. For the most significant bands the wave number $\tilde{\mathrm{v}}\left(\mathrm{cm}^{-1}\right)$ is given.

Chemicals were purchased from commercial suppliers. Unless stated otherwise, all the substrates and solvents were purified and dried according to standard methods prior to use. Reactions requiring inert conditions were carried out in glove box. 


\section{Experimental details}

\section{(1).General Procedure}<smiles>[R]C#[N+][C-]C[C@H](C)OCC</smiles>
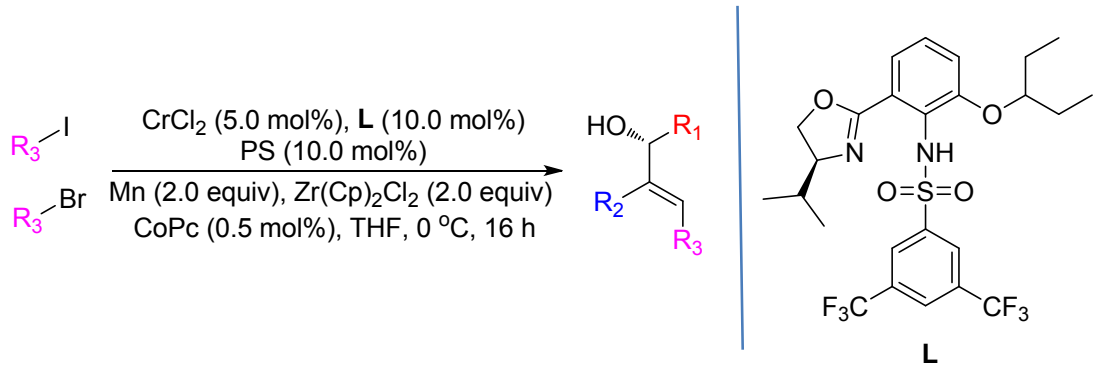

To a mixture of anhydrous chromium (II) chloride (1.2 mg, $0.01 \mathrm{mmol}, 5 \mathrm{~mol} \%)$, (S)N-(2-(4-isopropyl-4,5-dihydrooxazol-2-yl)-6-(pentan-3-yloxy)phenyl)-3,5bis(trifluoromethyl)benzenesulfonamide (L, $11.3 \mathrm{mg}, 0.02 \mathrm{mmol}, 10 \mathrm{~mol} \%)$ and Proton sponge (4.3 mg, $0.02 \mathrm{mmol}, 10 \mathrm{~mol} \%)$ was added THF $(1.0 \mathrm{~mL})$ under an nitrogen atmosphere. The mixture was stirred vigorously at room temperature for 2 hours. Then phthalocyaninecobalt (II) $(0.5 \mathrm{mg}, 0.001 \mathrm{mmol}, 0.5 \mathrm{~mol} \%)$ and manganese powder $(22.0$ mg, 0.4 mmol, 2.0 equiv.), $\mathrm{ZrCp}_{2} \mathrm{Cl}_{2}$ (117 mg, $0.4 \mathrm{mmol}, 2.0$ equiv.), aldehyde (0.2 mmol, 1.0 equiv.), alkyne ( $0.6 \mathrm{mmol}, 3.0$ equiv.) and alkyl iodides (0.4 mmol, 2.0 equiv.) were added in succession. The resulting suspension was stirred at $0{ }^{\circ} \mathrm{C}$ for $16 \mathrm{~h}$. After the full consumption of aldehyde, the mixture was diluted with ethyl acetate, stirred at room temperature for 15 minutes and the resulting suspension was filtered over a pad of silica gel using ethyl acetate (EtOAc) as eluent. Volatiles were evaporated in vacuo. The residue was purified by chromatography (Petroleum ether /ethyl acetate $=15 / 1$ to $10 / 1)$ afforded the product.

\section{(2). Synthesis and Characterization of Ligand and Products}

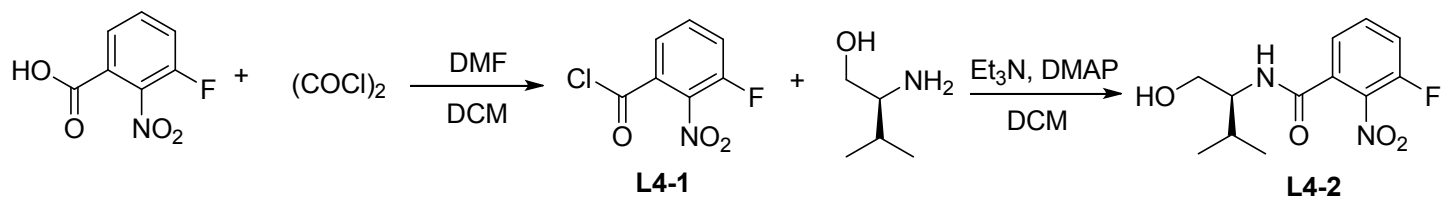

\section{Synthesis of L4-1}

To a $100 \mathrm{~mL}$ round bottom flask was added $1 \mathrm{~g}$ of 3-fluoro-2-nitrobenzoic acid (5.4 mmol, 1 equiv.), $1.2 \mathrm{ml}$ of Oxalyl chloride (10.8mmol, 2equiv.), $20 \mathrm{~mL}$ of methylene chloride, $0.1 \mathrm{ml}$ of DMF (75.6mmol, 1.4 equiv.). The reaction was stirred at room temperature for $3 \mathrm{~h}$. The solvent was removed in vacuo to afford crude L4-1, which was used for the next step without further purification

\section{Synthesis of L4-2}


To a $100 \mathrm{~mL}$ round bottom flask was added $669 \mathrm{mg}$ of (S)-2-amino-3-methylbutan-1-ol (6.5 mmol, 1.4 equiv.), $2.3 \mathrm{ml}$ of $\mathrm{Et}_{3} \mathrm{~N}$ (16.2mmol, 3equiv), $66 \mathrm{mg}$ of DMAP(0.54 mmol, 0.1 equiv), and $20 \mathrm{ml}$ of methylene chloride, and, after cooling to $0{ }^{\circ} \mathrm{C}, 1.0 \mathrm{~g}$ of L4$1(5.4 \mathrm{mmol}$, 1equiv) dissolved in 5ml DCM was slowly added into the cooled reaction mixture. The reaction was allowed to warm to room temperature overnight (12 h). The reaction mixture was filtered through celite which was then washed with additional methylene chloride. The combined organic phases were concentrated in vacuo. The product was separated by flash column chromatography (Petroleum ether : Ethyl acetate $=1: 1)$ as yellow/white solid (1.34 g, $4.96 \mathrm{mmol})$ in $92 \%$ yield.

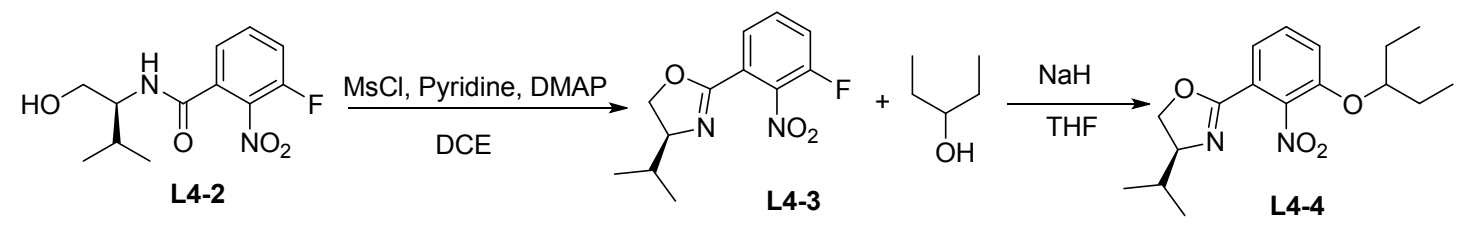

\section{Synthesis of L4-3}

To a $100 \mathrm{~mL}$ round bottom flask was added $1.3 \mathrm{~g}$ ( $4.8 \mathrm{mmol}, 1$ equiv. ) of the above yellow solid (S)-3-fluoro-N-(1-hydroxy-3-methylbutan-2-yl)-2-nitrobenzamide, $20 \mathrm{~mL}$ of methylene chloride and $15 \mathrm{~mL}$ of pyridine, after cooling to $0{ }^{\circ} \mathrm{C}, 825 \mathrm{mg}(7.2 \mathrm{mmol}$, 1.5 equiv.) of $\mathrm{MsCl}$. The reaction was warm to room temperature overnight ( $12 \mathrm{~h}$ ). The organic layer was washed with water and dried over anhydrous sodium sulfate. The L43 (S)-2-(3-fluoro-2-nitrophenyl)-4-isopropyl-4,5-dihydroox-azole (832 mg, $3.3 \mathrm{mmol}$ ) was purified by flash column chromatography (Petroleum ether : Ethyl acetate $=1: 1$ ), in $68.8 \%$ yield.

\section{Synthesis of L4-4}

To a $100 \mathrm{~mL}$ round bottom flask was added a slurry of $176 \mathrm{mg}$ of $\mathrm{NaH}$ (4.4 mmol, $60 \%$ in mineral oil, 3.6 equiv.) in anhydrous THF (15 mL) was added $150 \mathrm{mg}$ of 3-Pentanol ( $1.7 \mathrm{mmol}, 1.4$ equiv.) at $0{ }^{\circ} \mathrm{C}$ with stirring for $1 \mathrm{~h}$. To the cooled reaction mixture was slowly added $300 \mathrm{mg}$ of L4-3 (1.2 mmol, 1.0 equiv.) via syringe. The reaction was allowed to warm to room temperature and stirred for $12 \mathrm{~h}$, quenched with water at $0{ }^{\circ} \mathrm{C}$. The organic solvent (THF) was removed in vacuo and the slurry was extracted with ethyl acetate. The combined organic layers were washed with brine, water, dried over $\mathrm{MgSO}_{4}$ and filtered. The solvent was removed in vacuo to afford crude L4-4, which was used for the next step without further purification. 


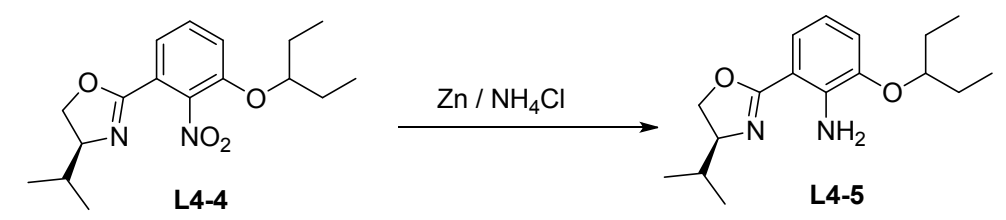

\section{Synthesis of L4-5}

To a $100 \mathrm{~mL}$ round bottom flask was added $460 \mathrm{mg}$ of L4-4 (1.44 mmol, 1.0 equiv.) in $20 \mathrm{ml}$ acetone, $373 \mathrm{mg}$ of zinc powder $(5.74 \mathrm{mmol}, 4.0$ equiv.) and $0.96 \mathrm{~mL}$ of $6 \mathrm{~mol} / \mathrm{L}$ ammonium chloride ( $5.74 \mathrm{mmol}, 4$ equiv.). The mixture was vigorously shaken at room temperature for $12 \mathrm{~h}$. The filtrate was diluted with EtOAc. The organic layer was collected, dried $\mathrm{MgSO}_{4}$, and concentrated to afford crude L4-5, then the crude product was purified by flash column chromatography (Petroleum ether : Ethyl acetate $=10: 1$ ), yellow solid (317.8 mg, $1.09 \mathrm{mmol})$, in $76 \%$ yield. ${ }^{1} \mathrm{H} \mathrm{NMR}\left(\mathrm{CDCl}_{3}, 400 \mathrm{MHz}\right) \delta 7.30$ $(\mathrm{d}, \mathrm{J}=8.0 \mathrm{~Hz}, 1 \mathrm{H}), 6.80(\mathrm{~d}, \mathrm{~J}=8.0 \mathrm{~Hz}, 1 \mathrm{H}), 6.56(\mathrm{t}, \mathrm{J}=8.0 \mathrm{~Hz}, 1 \mathrm{H}), 6.45(\mathrm{~s}, 2 \mathrm{H})$, $4.33(\mathrm{t}, \mathrm{J}=8.0 \mathrm{~Hz}, 1 \mathrm{H}), 4.16-4.09(\mathrm{~m}, 2 \mathrm{H}), 4.02(\mathrm{t}, \mathrm{J}=8.0 \mathrm{~Hz}, 1 \mathrm{H}), 1.84-1.76(\mathrm{~m}, 1 \mathrm{H})$, 1.74-1.67 (m, 4H), 1.04-0.93 (m, 12H). ${ }^{13} \mathrm{C} \mathrm{NMR}\left(\mathrm{CDCl}_{3}, 100 \mathrm{MHz}\right): \delta 164.0,145.4$, 140.6, 121.1, 114.6, 114.4, 110.2, 80.9, 72.9, 69.0, 33.3, 26.19, 26.16, 19.13, 18.71, $9.79,9.71$.
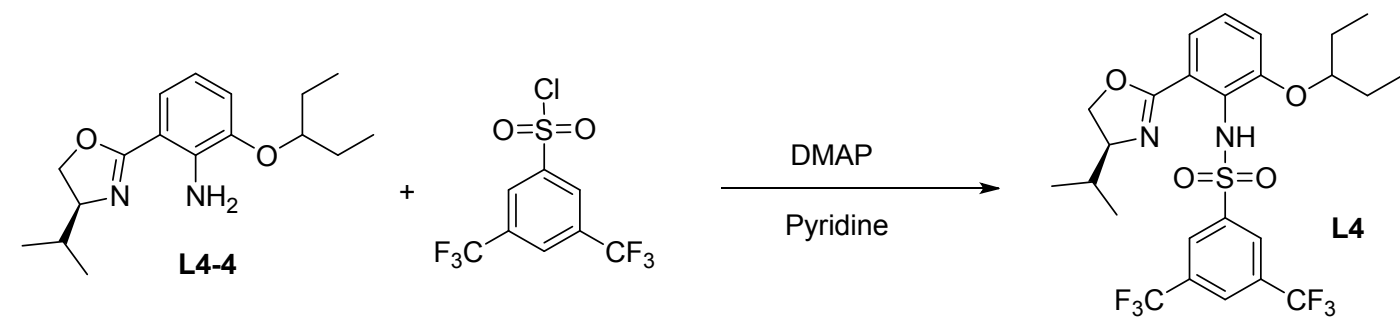

\section{Synthesis of Chiral Sulfonamide Ligand L4}

To a $50 \mathrm{~mL}$ round bottom flask was added $317.8 \mathrm{mg}$ of L 7-4 (1.09 mmol,1.0 equiv.)in anhydrous pyridine $(5 \mathrm{~mL}), 1.15 \mathrm{~g}$ of 3,4,5-trifluorobenzenesulfonyl chloride $(2.20$ mmol,2.0 equiv.) and $66 \mathrm{mg}$ of DMAP $(0.11 \mathrm{mmol}, 0.2$ equiv. $)$. The solution was stirred $8 \mathrm{~h}$ before quenched with water. The mixture was extracted with ethyl acetate and the combined organic layers were washed with $1 \mathrm{~N} \mathrm{HCl}$, brine and water. The organic layer was dried over $\mathrm{MgSO}_{4}$ and filtered. The crude product purified by chromatography (Petroleum ether: Ethyl acetate= 30:1)to afford pure product L4 (362 mg, $0.64 \mathrm{mmol})$, in $59 \%$ yield. ${ }^{1} \mathrm{H} \mathrm{NMR}\left(\mathrm{CDCl}_{3}, 400 \mathrm{MHz}\right) \delta 12.51(\mathrm{~s}, 1 \mathrm{H}), 8.46(\mathrm{~s}, 2 \mathrm{H}), 8.05(\mathrm{~s}, 1 \mathrm{H})$, $7.43(\mathrm{~d}, \mathrm{~J}=8.0 \mathrm{~Hz}, 1 \mathrm{H}), 7.13(\mathrm{t}, \mathrm{J}=8.0 \mathrm{~Hz}, 1 \mathrm{H}), 6.98(\mathrm{~d}, \mathrm{~J}=8.0 \mathrm{~Hz}, 1 \mathrm{H}), 7.42$ (t, J = $8.0 \mathrm{~Hz}, 1 \mathrm{H}), 4.18-4.10(\mathrm{~m}, 2 \mathrm{H}), 3.96-3.90(\mathrm{~m}, 1 \mathrm{H}), 1.91-1.82(\mathrm{~m}, 1 \mathrm{H}), 1.26-1.17(\mathrm{~m}$, 
2H), 1.09 (d, J = 8.0 Hz, 3H), 0.99 (d, J = 8.0 Hz, 3H), 0.93-0.83 (m, 1H), 0.74-0.61 $(\mathrm{m}, 7 \mathrm{H}) .{ }^{13} \mathrm{C} \mathrm{NMR}\left(\mathrm{CDCl}_{3}, 100 \mathrm{MHz}\right): \delta 163.7,151.7,146.2,132.3(\mathrm{q}, \mathrm{J}=34.1 \mathrm{~Hz})$, 128.6, 126.9 (q, $\mathrm{J}=2.6 \mathrm{~Hz}), 125.3,125.2(\mathrm{q}, \mathrm{J}=3.6 \mathrm{~Hz}), 122.8$ (q, $\mathrm{J}=271.6 \mathrm{~Hz}), 120.9$, $119.4,116.9,82.0,72.7,70.0,33.3,26.07,25.91,18.86,18.74,9.95,9.85 .{ }^{19} \mathrm{~F}$ NMR $(376 \mathrm{MHz}, \mathrm{CDCl} 3) \delta-62.922(\mathrm{~s}, 6 \mathrm{~F})$.

This ligand (L) was synthesized according to the known literature procedure. ${ }^{1}$

\section{Characterization data of products}

\section{(R,E)-6,6-dimethyl-1,4-diphenylhept-4-en-3-ol (1d)}<smiles>CC(C)(C)/C=C(/c1ccccc1)[C@H](O)CCc1ccccc1</smiles>

According to general procedure, $\mathbf{1 d}(47.7 \mathrm{mg}, 0.16 \mathrm{mmol})$ was obtained as colorless oil in $81 \%$ yield after chromatography (silica gel, eluent petroleum ether /ethyl acetate $=$ $15 / 1$ to $10 / 1) .{ }^{2}{ }^{1} \mathrm{H} \mathrm{NMR}\left(\mathrm{CDCl}_{3}, 400 \mathrm{MHz}\right) \delta$ 7.32-7.24 (m, 5H), 7.18-7.12 (m, 5H), $5.64(\mathrm{~s}, 1 \mathrm{H}), 4.16-4.11(\mathrm{~m}, 1 \mathrm{H}), 2.79-2.62(\mathrm{~m}, 2 \mathrm{H}), 1.82-1.65(\mathrm{~m}, 2 \mathrm{H}), 1.54-1.53(\mathrm{~m}$, $1 \mathrm{H}), 0.86(\mathrm{~s}, 9 \mathrm{H}) ;{ }^{13} \mathrm{C} \mathrm{NMR}\left(\mathrm{CDCl}_{3}, 100 \mathrm{MHz}\right): \delta 142.1,140.2,139.3,138.1,130.2$, $128.6,128.5,127.7,127.0,125.9,78.1,37.2,33.5,32.4,31.4$. IR (neat) $\mathrm{cm}^{-1}$ ṽ: 3332 , 2958, 2927, 2857, 1494, 1453, 1410, 1260, 1088, 1053, 1019, 876, 797, 740, 722, 699, 576, 499. HRMS (EI(+), $70 \mathrm{eV}): \mathrm{C}_{21} \mathrm{H}_{26} \mathrm{O}$ [M]: calcd: 294.1984, found: 294.1992. HPLC (Chiralcel OD-H column, hexanes: $i-\mathrm{PrOH}=95: 5,1.0 \mathrm{~mL} / \mathrm{min}, 210 \mathrm{~nm}$ ), $\mathrm{t}_{\text {minor }}=$ $5.7 \mathrm{~min}, \mathrm{t}_{\text {major }}=9.0 \mathrm{~min}$, ee $=94 \% .[\alpha]_{\mathrm{D}}^{29}=-7.5,\left(\mathrm{c}=1.3, \mathrm{CH}_{2} \mathrm{Cl}_{2}\right)$.

(R,E)-2,2-dimethyl-4-phenyldec-3-en-5-ol (2d)

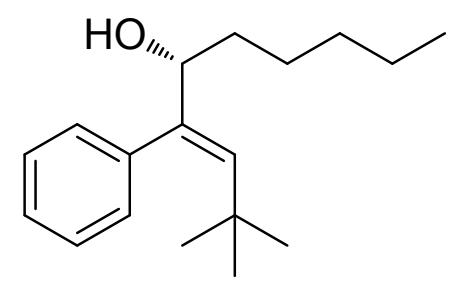

According to general procedure, $2 \mathbf{d}(41.1 \mathrm{mg}, 0.16 \mathrm{mmol})$ was obtained as colorless oil in $79 \%$ yield after chromatography (silica gel, eluent petroleum ether /ethyl acetate $=$ 
15/1 to $10 / 1) .{ }^{1} \mathrm{H} \mathrm{NMR}\left(\mathrm{CDCl}_{3}, 400 \mathrm{MHz}\right) \delta$ 7.31-7.26 (m, 3H), 7.12-7.10 (m, 2H), $5.62(\mathrm{~s}, 1 \mathrm{H}), 4.12-4.04(\mathrm{~m}, 1 \mathrm{H}), 1.48-1.28(\mathrm{~m}, 9 \mathrm{H}), 0.89-0.85(\mathrm{~m}, 12 \mathrm{H}) ;{ }^{13} \mathrm{C} \mathrm{NMR}$ $\left(\mathrm{CDCl}_{3}, 100 \mathrm{MHz}\right): \delta 140.4,138.9,138.3,130.3,127.6,126.9,78.8,35.6,33.4,31.9$, $31.4,25.7,22.8,14.2$. IR (neat) $\mathrm{cm}^{-1} \tilde{\mathrm{v}}: 3347,2955,2930,2860,1462,1442,1308,1260$, 1159, 1124, 1027, 951, 875, 770, 703, 642, 499. HRMS (EI(+), $70 \mathrm{eV}): \mathrm{C}_{18} \mathrm{H}_{28} \mathrm{O}[\mathrm{M}]$ : calcd: 260.2140, found: 260.2141. HPLC (Chiralcel OD-H column, hexanes: $i-\mathrm{PrOH}=$ $95: 5,0.80 \mathrm{~mL} / \mathrm{min}, 210 \mathrm{~nm}), \mathrm{t}_{\text {minor }}=4.4 \mathrm{~min}, \mathrm{t}_{\text {major }}=4.7 \mathrm{~min}$, ee $=94 \% .[\alpha]_{\mathrm{D}}{ }^{29}=-11.3$, (c $=0.5, \mathrm{CH}_{2} \mathrm{Cl}_{2}$ ).

\section{(R,E)-1-(2,2-dimethyl-1,1-diphenylpropoxy)-7,7-dimethyl-5-phenyloct-5-en-4-ol} (3d)

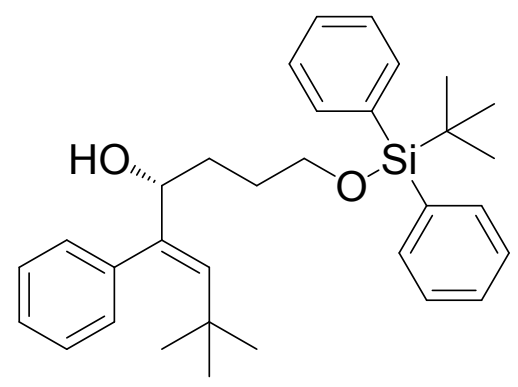

According to general procedure, $\mathbf{3 d}(73.0 \mathrm{mg}, 0.15 \mathrm{mmol})$ was obtained as colorless oil in $75 \%$ yield after chromatography (silica gel, eluent petroleum ether /ethyl acetate $=$ 15/1 to $10 / 1) .{ }^{1} \mathrm{H} \mathrm{NMR}\left(\mathrm{CDCl}_{3}, 400 \mathrm{MHz}\right) \delta$ 7.65-7.64 (m, 4H), 7.44-7.35 (m, 6H), 7.30-7.26 (m, 3H), 7.11-7.09 (m, 2H), $5.65(\mathrm{~s}, 1 \mathrm{H}), 4.12(\mathrm{t}, J=8.0 \mathrm{~Hz}, 1 \mathrm{H}), 3.69-3.60$ $(\mathrm{m}, 2 \mathrm{H}), 1.95(\mathrm{~s}, 1 \mathrm{H}), 1.68-1.56(\mathrm{~m}, 4 \mathrm{H}), 1.50-1.39(\mathrm{~m}, 1 \mathrm{H}), 1.03$ (s, 9H), $0.86(\mathrm{~s}, 9 \mathrm{H}) ;{ }^{13} \mathrm{C} \mathrm{NMR}\left(\mathrm{CDCl}_{3}, 100 \mathrm{MHz}\right): \delta 140.3,138.7,138.5,135.7,133.9$, $130.3,129.7,127.7,127.6,126.9,78.3,64.1,33.4,32.2,31.4,28.8,27.0,19.3$. IR (neat) $\mathrm{cm}^{-1} \tilde{\mathrm{v}}: 3341,2930,2899,2857,1443,1388,1360,1261,1191,1107,1083,1020,821$, 799, 739, 701, 611, 502. HRMS (EI(+), $70 \mathrm{eV}): \mathrm{C}_{32} \mathrm{H}_{42} \mathrm{O}_{2} \mathrm{Si}[\mathrm{M}]$ : calcd: 486.2954, found: 486.2949. HPLC (Chiralcel OD-H column, hexanes: $i$-PrOH $=98: 12,0.8$ $\mathrm{mL} / \mathrm{min}, 210 \mathrm{~nm}), \mathrm{t}_{\text {minor }}=5.6 \mathrm{~min}, \mathrm{t}_{\text {major }}=6.0 \mathrm{~min}$, ee $=93 \% .[\alpha]_{\mathrm{D}}{ }^{29}=-6.2,(\mathrm{c}=0.6$, $\mathrm{CH}_{2} \mathrm{Cl}_{2}$ ).

\section{(R,E)-6-methyl-1,4-diphenylhept-4-en-3-ol (4d)}<smiles>CC(C)/C=C(\c1ccccc1)[C@H](O)CCc1ccccc1</smiles> 
According to general procedure, $\mathbf{4 d}(39.2 \mathrm{mg}, 0.14 \mathrm{mmol})$ was obtained as colorless oil in $70 \%$ yield after chromatography (silica gel, eluent petroleum ether /ethyl acetate $=$ 15/1 to $10 / 1) .{ }^{1} \mathrm{H} \mathrm{NMR}\left(\mathrm{CDCl}_{3}, 400 \mathrm{MHz}\right) \delta$ 7.36-7.23 (m, 5H), 7.18-7.13 (m, 5H), $5.50(\mathrm{~d}, J=12.0 \mathrm{~Hz}, 1 \mathrm{H}), 4.30-4.25(\mathrm{~m}, 1 \mathrm{H}), 2.78-2.60(\mathrm{~m}, 2 \mathrm{H}), 2.29-2.19(\mathrm{~m}, 1 \mathrm{H})$, 1.83-1.69 (m, 2H), 1.66-1.61 (m, 1H), $0.92(\mathrm{dd}, J=4.0 \mathrm{~Hz}, 6 \mathrm{H}) ;{ }^{13} \mathrm{C} \mathrm{NMR}\left(\mathrm{CDCl}_{3}, 100\right.$ MHz): $\delta 142.1,140.8,138.4,136.5,129.3,128.6,128.4,128.3,127.0,125.9,76.2,37.3$, 32.2, 27.8, 23.3. IR (neat) $\mathrm{cm}^{-1} \tilde{\mathrm{v}}: 3357,3026,2954,2927,2865,1601,1494,1455$, 1360, 1261, 1071, 1054, 1030, 944, 917, 881, 768, 747, 700, 506. HRMS (EI(+), 70 eV): $\mathrm{C}_{20} \mathrm{H}_{24} \mathrm{O}$ [M]: calcd: 280.1827, found: 280.1833. HPLC (Chiralcel OD-H column, hexanes: $i-\mathrm{PrOH}=90: 10,0.80 \mathrm{~mL} / \mathrm{min}, 210 \mathrm{~nm}), \mathrm{t}_{\text {minor }}=5.7 \mathrm{~min}$, $\mathrm{t}_{\text {major }}=7.5 \mathrm{~min}$, ee $=$ $95 \% .[\alpha]_{\mathrm{D}}^{30}=-3.9,\left(\mathrm{c}=0.3, \mathrm{CH}_{2} \mathrm{Cl}_{2}\right)$.

\section{(R,E)-2-methyl-4-phenyldec-3-en-5-ol (5d)}

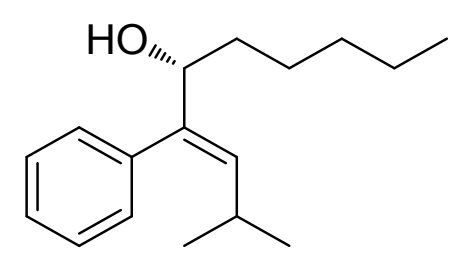

According to general procedure, $\mathbf{5 d}(34.5 \mathrm{mg}, 0.14 \mathrm{mmol})$ was obtained as colorless oil in $70 \%$ yield after chromatography (silica gel, eluent petroleum ether /ethyl acetate $=$ 15/1 to $10 / 1) .{ }^{1} \mathrm{H}$ NMR $\left(\mathrm{CDCl}_{3}, 400 \mathrm{MHz}\right) \delta$ 7.36-7.26 (m, 3H), 7.15-7.13 (m, 2H), $5.47(\mathrm{~d}, J=8.0 \mathrm{~Hz}, 1 \mathrm{H}), 4.26-4.20(\mathrm{~m}, 1 \mathrm{H}), 2.26-2.17(\mathrm{~m}, 1 \mathrm{H}), 1.54(\mathrm{~s}, 1 \mathrm{H}), 1.46-1.26$

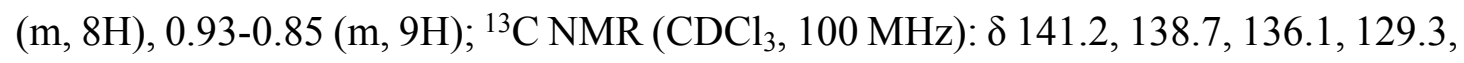
128.2, 126.9, 76.9, 35.7, 31.8, 27.8, 25.6, 23.3, 22.8, 14.2. IR (neat) $\mathrm{cm}^{-1} \tilde{\mathrm{v}}: 3354,2955$, 2929, 2862, 1493, 1463, 1379, 1361, 1305, 1260, 1126, 1104, 1072, 1206, 947, 878, 770, 702, 664, 488. HRMS (EI(+), $70 \mathrm{eV}): \mathrm{C}_{17} \mathrm{H}_{26} \mathrm{O}$ [M]: calcd: 246.1984, found: 246.1991. HPLC (Chiralcel OD-H column, hexanes: $i$-PrOH $=95: 5,0.80 \mathrm{~mL} / \mathrm{min}, 210$ $\mathrm{nm}), \mathrm{t}_{\text {minor }}=4.5 \mathrm{~min}, \mathrm{t}_{\text {major }}=4.7 \mathrm{~min}, \mathrm{ee}=95 \% .[\alpha]_{\mathrm{D}}{ }^{30}=-1.8,\left(\mathrm{c}=0.2, \mathrm{CH}_{2} \mathrm{Cl}_{2}\right)$.

\section{(R,E)-3-cyclohexyl-1,2-diphenylprop-2-en-1-ol (6d)}<smiles>CC(C)/C=C(\c1ccccc1)[C@H](O)c1ccccc1</smiles>

According to general procedure, $\mathbf{6 d}(31.8 \mathrm{mg}, 0.13 \mathrm{mmol})$ was obtained as colorless oil in $63 \%$ yield after chromatography (silica gel, eluent petroleum ether /ethyl acetate = 
15/1 to 5/1). ${ }^{1} \mathrm{H}$ NMR $\left(\mathrm{CDCl}_{3}, 400 \mathrm{MHz}\right) \delta$ 7.34-7.20 (m, 8H), 6.90-6.88 (m, 2H), 5.69 $(\mathrm{d}, J=8.0 \mathrm{~Hz}, 1 \mathrm{H}), 5.39-5.36(\mathrm{~m}, 1 \mathrm{H}), 2.01-1.96(\mathrm{~m}, 1 \mathrm{H}), 1.63-1.56(\mathrm{~m}, 6 \mathrm{H}), 1.17-1.11$ (m, 5H); ${ }^{13} \mathrm{C} \mathrm{NMR}\left(\mathrm{CDCl}_{3}, 100 \mathrm{MHz}\right): \delta 142.3,140.7,138.2,136.5,129.5,128.3,128.0$, 127.5, 127.0, 126.8, 78.6, 27.9, 23.3. IR (neat) $\mathrm{cm}^{-1} \tilde{\mathrm{v}}: 3389,2956,2926,2865,1492$, 1452, 1361, 1260, 1172, 1062, 1029, 1007, 948, 818, 758, 699, 604, 524. HRMS (EI(+), $70 \mathrm{eV}$ ): $\mathrm{C}_{18} \mathrm{H}_{20} \mathrm{O}[\mathrm{M}]:$ calcd: 252.1514, found: 252.1512. HPLC (Chiralcel OD-H column, hexanes: $i-\mathrm{PrOH}=90: 10,0.80 \mathrm{~mL} / \mathrm{min}, 210 \mathrm{~nm}), \mathrm{t}_{\text {minor }}=5.3 \mathrm{~min}, \mathrm{t}_{\text {major }}=5.8$ $\min$, ee $=96 \%$. $[\alpha]_{\mathrm{D}}{ }^{30}=-23.2,\left(\mathrm{c}=0.7, \mathrm{CH}_{2} \mathrm{Cl}_{2}\right)$.

\section{(R,E)-4,4-dimethyl-1,2-diphenylpent-2-en-1-ol (7d)}<smiles>CC(C)(C)/C=C(/c1ccccc1)[C@@H](O)c1ccccc1</smiles>

According to general procedure, $7 \mathbf{d}(36.2 \mathrm{mg}, 0.14 \mathrm{mmol})$ was obtained as colorless oil in $68 \%$ yield after chromatography (silica gel, eluent petroleum ether /ethyl acetate $=$ 15/1 to 5/1). ${ }^{1} \mathrm{H}$ NMR $\left(\mathrm{CDCl}_{3}, 400 \mathrm{MHz}\right) \delta$ 7.28-7.22 (m, 3H), 7.20-7.12 (m, 5H), 6.79$6.76(\mathrm{~m}, 2 \mathrm{H}), 5.9(\mathrm{~s}, 1 \mathrm{H}), 5.26(\mathrm{~d}, J=4.0 \mathrm{~Hz}, 1 \mathrm{H}), 2.03(\mathrm{~d}, J=4.0 \mathrm{~Hz}, 1 \mathrm{H}), 0.88(\mathrm{~s}$, $9 \mathrm{H}) ;{ }^{13} \mathrm{C} \mathrm{NMR}\left(\mathrm{CDCl}_{3}, 100 \mathrm{MHz}\right): \delta 142.2,140.2,138.8,137.8,130.4,128.1,127.4$, 127.3, 126.9, 126.6, 80.3, 33.6, 31.4. IR (neat) $\mathrm{cm}^{-1} \tilde{\mathrm{v}}: 3370,2955,2901,2865,1492$, 1452, 1361, 1219, 1068, 1028, 949, 916, 805, 762, 699, 611, 523, 498. HRMS (EI(+), $70 \mathrm{eV}$ ): $\mathrm{C}_{19} \mathrm{H}_{22} \mathrm{O}$ [M]: calcd: 266.1671, found: 266.1674. HPLC (Chiralcel OD-H column, hexanes: $i-\mathrm{PrOH}=90: 10,0.80 \mathrm{~mL} / \mathrm{min}, 210 \mathrm{~nm}), \mathrm{t}_{\text {minor }}=5.0 \mathrm{~min}, \mathrm{t}_{\text {major }}=5.5$ $\min$, ee $=94 \%$. $[\alpha]_{\mathrm{D}}{ }^{29}=-27.3,\left(\mathrm{c}=0.6, \mathrm{CH}_{2} \mathrm{Cl}_{2}\right)$.

\section{(R,E)-1-(4-bromophenyl)-4-methyl-2-phenylpent-2-en-1-ol (8d)}<smiles>CC(C)/C=C(\c1ccccc1)[C@H](O)c1ccc(Br)cc1</smiles>

According to general procedure, $\mathbf{8 d}(38.4 \mathrm{mg}, 0.12 \mathrm{mmol})$ was obtained as colorless oil in $58 \%$ yield after chromatography (silica gel, eluent petroleum ether /ethyl acetate $=$ 
10/1 to 3/1). ${ }^{1} \mathrm{H} \mathrm{NMR}\left(\mathrm{CDCl}_{3}, 400 \mathrm{MHz}\right) \delta$ 7.41-7.38 (m, 2H), 7.24-7.22 (m, 3H), 7.13$7.11(\mathrm{~m}, 2 \mathrm{H}), 6.90-6.87(\mathrm{~m}, 2 \mathrm{H}), 5.65(\mathrm{~d}, J=8.0 \mathrm{~Hz}, 1 \mathrm{H}), 5.34(\mathrm{~d}, J=4.0 \mathrm{~Hz}, 1 \mathrm{H})$, 2.27-2.23 (m, 1H), $1.99(\mathrm{~d}, J=4.0 \mathrm{~Hz}, 1 \mathrm{H}), 0.93(\mathrm{dd}, J=8.0,8.0 \mathrm{~Hz}, 6 \mathrm{H}) ;{ }^{13} \mathrm{C} \mathrm{NMR}$ $\left(\mathrm{CDCl}_{3}, 100 \mathrm{MHz}\right): \delta 141.29,140.44,137.68,136.97,131.32,129.42,128.44,128.13$, 127.18, 121.31, 78.09, 27.89, 23.2. IR (neat) $\mathrm{cm}^{-1} \tilde{\mathrm{v}}: 3382,2957,2925,2865,1667$, 1592, 1486, 1463, 1442, 1400, 1291, 1260, 1171, 1069, 1030, 1008, 947, 803, 768, 701, 607, 525. HRMS (EI(+), $70 \mathrm{eV}): \mathrm{C}_{18} \mathrm{H}_{19} \mathrm{OBr}$ [M]: calcd: 330.0619, found: 330.0618 . HPLC (Chiralcel OD-H column, hexanes: $i-\mathrm{PrOH}=95: 5,1.0 \mathrm{~mL} / \mathrm{min}, 210 \mathrm{~nm}$ ), $\mathrm{t}_{\text {minor }}=$ $5.5 \mathrm{~min}, \mathrm{t}_{\text {major }}=6.4 \mathrm{~min}$, ee $=96 \% .[\alpha]_{\mathrm{D}}{ }^{30}=-21.4,\left(\mathrm{c}=0.9, \mathrm{CH}_{2} \mathrm{Cl}_{2}\right)$.

\section{(R,E)-4-methyl-2-phenyl-1-(m-tolyl)pent-2-en-1-ol (9d)}<smiles>Cc1cccc([C@H](O)/C(=C/C(C)C)c2ccccc2)c1</smiles>

According to general procedure, $9 \mathbf{d}(32.5 \mathrm{mg}, 0.12 \mathrm{mmol})$ was obtained as colorless oil in $61 \%$ yield after chromatography (silica gel, eluent petroleum ether /ethyl acetate $=$ 15/1 to 5/1). ${ }^{1} \mathrm{H}$ NMR $\left(\mathrm{CDCl}_{3}, 400 \mathrm{MHz}\right) \delta$ 7.25-7.15 (m, 4H), 7.07-7.03 (m, 3H), 6.93$6.90(\mathrm{~m}, 2 \mathrm{H}), 5.67(\mathrm{~d}, J=12.0 \mathrm{~Hz}, 1 \mathrm{H}), 5.35-5.31(\mathrm{~m}, 1 \mathrm{H}), 2.30(\mathrm{~s}, 3 \mathrm{H}), 1.95(\mathrm{~s}, 1 \mathrm{H})$, $0.94(\mathrm{~d}, J=8.0,8.0 \mathrm{~Hz}, 6 \mathrm{H}) ;{ }^{13} \mathrm{C} \mathrm{NMR}\left(\mathrm{CDCl}_{3}, 100 \mathrm{MHz}\right): \delta 142.2,140.6,138.3$, $137.8,136.4,129.5,128.2$, 128.1, 128.0, 127.5, 127.0, 123.9, 78.6, 27.9, 23.2, 21.6. IR (neat) $\mathrm{cm}^{-1} \tilde{\mathrm{v}}: 3363,2956,2925,2866,1605,1490,1462,1442,1361,1307,1260,1151$, 1067, 1014, 949, 882, 795, 775, 702, 623, 549. HRMS (EI(+), $70 \mathrm{eV}): \mathrm{C}_{19} \mathrm{H}_{22} \mathrm{O}[\mathrm{M}]$ : calcd: 266.1671, found: 266.1667. HPLC (Chiralcel OD-H column, hexanes: $i-\mathrm{PrOH}=$ $95: 5,1.0 \mathrm{~mL} / \mathrm{min}, 210 \mathrm{~nm}), \mathrm{t}_{\text {minor }}=5.0 \mathrm{~min}, \mathrm{t}_{\text {major }}=5.8 \mathrm{~min}$, ee $=95 \% .[\alpha]_{\mathrm{D}}{ }^{30}=-21.7$, (c $=1.5, \mathrm{CH}_{2} \mathrm{Cl}_{2}$ ).

\section{(R,E)-6-methyl-1-phenyl-4-(p-tolyl)hept-4-en-3-ol (10d)}<smiles>Cc1ccc(/C(=C\C(C)C)[C@H](O)CCc2ccccc2)cc1</smiles>

According to general procedure, $10 \mathrm{~d}(41.8 \mathrm{mg}, 0.14 \mathrm{mmol})$ was obtained as colorless 
oil in $71 \%$ yield after chromatography (silica gel, eluent petroleum ether /ethyl acetate $=15 / 1$ to $10 / 1) .{ }^{1} \mathrm{H} \mathrm{NMR}\left(\mathrm{CDCl}_{3}, 400 \mathrm{MHz}\right) \delta 7.27-7.23(\mathrm{~m}, 3 \mathrm{H}), 7.18-7.14(\mathrm{~m}, 5 \mathrm{H})$, 7.05-7.04 (m, 2H), $5.48(\mathrm{~d}, J=12.0 \mathrm{~Hz}, 1 \mathrm{H}), 4.28-4.24(\mathrm{~m}, 1 \mathrm{H}), 2.77-2.60(\mathrm{~m}, 2 \mathrm{H})$, $2.35(\mathrm{~s}, 3 \mathrm{H}), 2.30-2.21(\mathrm{~m}, 1 \mathrm{H}), 1.83-1.66(\mathrm{~m}, 2 \mathrm{H}), 1.59(\mathrm{~s}, 1 \mathrm{H}), 0.92(\mathrm{dd}, J=4.0,4.0$ $\mathrm{Hz}, 6 \mathrm{H}) ;{ }^{13} \mathrm{C} \mathrm{NMR}\left(\mathrm{CDCl}_{3}, 100 \mathrm{MHz}\right): \delta$ 142.2, 140.8, 136.6, 136.4, 135.3, 129.2, $129.0,128.6,128.4,125.9,76.3,37.3,32.2,27.8,23.3,21.3$. IR (neat) $\mathrm{cm}^{-1} \tilde{\mathrm{v}}: 3365$, 3025, 2954, 2924, 2864, 1670, 1603, 1511, 1495, 1454, 1360, 1261, 1092, 1052, 1027 , 945, 880, 821, 746, 698, 545, 503. HRMS (EI(+), $70 \mathrm{eV}): \mathrm{C}_{21} \mathrm{H}_{26} \mathrm{O}[\mathrm{M}]:$ calcd: 294.1984, found: 294.1985. HPLC (Chiralcel OD-H column, hexanes: $i-\mathrm{PrOH}=90: 10$, $1.0 \mathrm{~mL} / \mathrm{min}, 210 \mathrm{~nm}), \mathrm{t}_{\text {minor }}=4.9 \mathrm{~min}, \mathrm{t}_{\text {major }}=6.3 \mathrm{~min}, \mathrm{ee}=91 \% .[\alpha]_{\mathrm{D}}{ }^{30}=-5.4,(\mathrm{c}=0.2$, $\mathrm{CH}_{2} \mathrm{Cl}_{2}$ ).

\section{(R,E)-4-(4-methoxyphenyl)-6-methyl-1-phenylhept-4-en-3-ol (11d)}<smiles>COc1ccc(/C(=C\C(C)C)[C@H](O)CCc2ccccc2)cc1</smiles>

According to general procedure, 11d $(43.5 \mathrm{mg}, 0.14 \mathrm{mmol})$ was obtained as colorless oil in $70 \%$ yield after chromatography (silica gel, eluent petroleum ether /ethyl acetate $=15 / 1$ to $10 / 1) .{ }^{1} \mathrm{H} \mathrm{NMR}\left(\mathrm{CDCl}_{3}, 400 \mathrm{MHz}\right) \delta 7.25-7.23(\mathrm{~m}, 2 \mathrm{H}), 7.18-7.13(\mathrm{~m}, 3 \mathrm{H})$, 6.89-6.87 (m, 2H), 5.47 (d, $J=8.0 \mathrm{~Hz}, 1 \mathrm{H}), 4.27-4.23(\mathrm{~m}, 1 \mathrm{H}), 3.81$ (s, 3H), 2.80-2.59 (m, 2H), 2.31-2.22 (m, 1H), 1.82-1.66 (m, 2H), 1.61 (s, 1H), 0.92 (dd, $J=4.0,4.0 \mathrm{~Hz}$, $6 \mathrm{H}) ;{ }^{13} \mathrm{C} \mathrm{NMR}\left(\mathrm{CDCl}_{3}, 100 \mathrm{MHz}\right): \delta 158.6,142.2,140.4,136.6,130.6,130.3,128.6$, $128.4,125.9,113.7,76.4,55.3,37.3,32.2,27.8,23.3$. IR (neat) $\mathrm{cm}^{-1} \tilde{\mathrm{v}}: 3402,2954$, 2930, 2864, 1606, 1510, 1284, 1243, 1176, 1092, 1032, 880, 834, 801, 748, 699, 523. HRMS $(\mathrm{EI}(+), 70 \mathrm{eV}): \mathrm{C}_{21} \mathrm{H}_{26} \mathrm{O}_{2}[\mathrm{M}]$ : calcd: 310.1933, found: 310.1938. HPLC (Chiralcel OD-H column, hexanes: $i-\mathrm{PrOH}=90: 10,1.0 \mathrm{~mL} / \mathrm{min}, 210 \mathrm{~nm}$ ), $\mathrm{t}_{\text {minor }}=5.7$ $\min , \mathrm{t}_{\text {major }}=7.7 \mathrm{~min}$, ee $=92 \% .[\alpha]_{\mathrm{D}}^{30}=1.9,\left(\mathrm{c}=0.2, \mathrm{CH}_{2} \mathrm{Cl}_{2}\right)$.

\section{(R,E)-4-(4-(tert-butyl)phenyl)-6-methyl-1-phenylhept-4-en-3-ol (12d)}




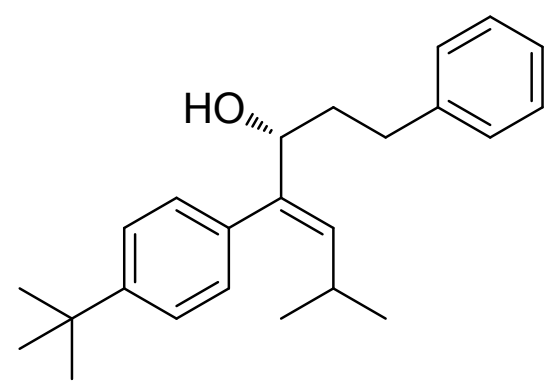

According to general procedure, $\mathbf{1 2 d}(45.1 \mathrm{mg}, 0.13 \mathrm{mmol})$ was obtained as colorless oil in 67\% yield after chromatography (silica gel, eluent petroleum ether /ethyl acetate $=15 / 1$ to $10 / 1) .{ }^{1} \mathrm{H} \mathrm{NMR}\left(\mathrm{CDCl}_{3}, 400 \mathrm{MHz}\right) \delta 7.35-7.31(\mathrm{~m}, 2 \mathrm{H}), 7.27-7.23(\mathrm{~m}, 2 \mathrm{H})$, 7.18-7.14 (m, 3H), 7.08-7.06 (m, 2H), $5.48(\mathrm{~d}, J=12.0 \mathrm{~Hz}, 1 \mathrm{H}), 4.29-4.23(\mathrm{~m}, 1 \mathrm{H})$, $2.78-2.23(\mathrm{~m}, 2 \mathrm{H}), 2.32-2.23(\mathrm{~m}, 1 \mathrm{H}), 1.84-1.68(\mathrm{~m}, 2 \mathrm{H}), 1.33(\mathrm{~s}, 9 \mathrm{H}), 0.92(\mathrm{dd}, J=$ 4.0, 4.0 Hz, 6H); ${ }^{13} \mathrm{C} \mathrm{NMR}\left(\mathrm{CDCl}_{3}, 100 \mathrm{MHz}\right): \delta 149.7,142.3,140.7,136.4,135.2$, $128.9,128.6,128.4,125.9,125.1,76.3,37.3,34.6,32.2,31.5,27.7,23.4$. IR (neat) $\mathrm{cm}^{-1}$ ṽ: 3344, 2958, 2866, 1603, 1496, 1457, 1362, 1304, 1268, 1160, 1094, 1055, 1026, 949 , 881, 816, 781, 746, 698, 652, 554, 492. HRMS (EI(+), 70 eV): $\mathrm{C}_{24} \mathrm{H}_{32} \mathrm{O}$ [M]: calcd: 336.2453, found: 336.2442. HPLC (Chiralcel OD-H column, hexanes: $i-\mathrm{PrOH}=90: 10$, $1.0 \mathrm{~mL} / \mathrm{min}, 210 \mathrm{~nm}), \mathrm{t}_{\text {minor }}=4.3 \mathrm{~min}, \mathrm{t}_{\text {major }}=5.3 \mathrm{~min}$, ee $=91 \% .[\alpha]_{\mathrm{D}}{ }^{30}=0.9,(\mathrm{c}=0.3$, $\mathrm{CH}_{2} \mathrm{Cl}_{2}$ ).

\section{(R,E)-4-(3-chlorophenyl)-6-methyl-1-phenylhept-4-en-3-ol (13d)}<smiles>CC(C)/C=C(/c1cccc(Cl)c1)[C@H](O)CCc1ccccc1</smiles>

According to general procedure, 13d $(35.3 \mathrm{mg}, 0.11 \mathrm{mmol})$ was obtained as colorless oil in 56\% yield after chromatography (silica gel, eluent petroleum ether /ethyl acetate $=15 / 1$ to $5 / 1) .{ }^{1} \mathrm{H} \mathrm{NMR}\left(\mathrm{CDCl}_{3}, 400 \mathrm{MHz}\right) \delta$ 7.27-7.24 (m, 4H), 7.19-7.13 (m, 4H), 7.05-7.13 (m, 1H), $5.52(\mathrm{~d}, J=12.0 \mathrm{~Hz}, 1 \mathrm{H}), 4.28-4.22(\mathrm{~m}, 1 \mathrm{H}), 2.77-2.60(\mathrm{~m}, 2 \mathrm{H})$, 2.27-2.18 (m, 1H), 1.80-1.67 (m, 2H), 0.92-0.90 (m, 6H); ${ }^{13} \mathrm{C} \mathrm{NMR}\left(\mathrm{CDCl}_{3}, 100 \mathrm{MHz}\right)$ : $\delta 141.9,140.3,139.7,137.4,134.1,129.5,129.3,128.6,128.5,127.6,127.3,126.0$, 76.0, 37.2, 32.2, 27.8, 23.2. IR (neat) $\mathrm{cm}^{-1} \tilde{\mathrm{v}}: 3356,2955,2928,2865,1592,1562,1495$, 1470, 1454, 1407, 1361, 1261, 1169, 1077, 1053, 1027, 948, 883, 798, 747, 698, 520. HRMS (EI(+), $70 \mathrm{eV}): \mathrm{C}_{20} \mathrm{H}_{23} \mathrm{OCl}[\mathrm{M}]:$ calcd: 314.1437, found: 314.1429. HPLC (Chiralcel OD-H column, hexanes: $i-\mathrm{PrOH}=90: 10,1.0 \mathrm{~mL} / \mathrm{min}, 210 \mathrm{~nm}$ ), $\mathrm{t}_{\text {minor }}=4.7$ $\min , \mathrm{t}_{\text {major }}=6.5 \mathrm{~min}, \mathrm{ee}=95 \% .[\alpha]_{\mathrm{D}}^{30}=5.0,\left(\mathrm{c}=0.3, \mathrm{CH}_{2} \mathrm{Cl}_{2}\right)$. 
<smiles>CC(C)/C=C(/c1ccc(Br)cc1)[C@H](O)CCc1ccccc1</smiles>

According to general procedure, $\mathbf{1 4 d}(41.7 \mathrm{mg}, 0.12 \mathrm{mmol})$ was obtained as colorless oil in 58\% yield after chromatography (silica gel, eluent petroleum ether /ethyl acetate $=15 / 1$ to $10 / 1) .{ }^{1} \mathrm{H} \mathrm{NMR}\left(\mathrm{CDCl}_{3}, 400 \mathrm{MHz}\right) \delta 7.47-7.45(\mathrm{~m}, 2 \mathrm{H}), 7.27-7.24(\mathrm{~m}, 2 \mathrm{H})$, 7.18-7.12 (m, 3H), 7.04-7.02 (m, 2H), $5.51(\mathrm{~d}, J=12.0 \mathrm{~Hz}, 1 \mathrm{H}), 4.26-4.22(\mathrm{~m}, 1 \mathrm{H})$, 2.76-2.58 (m, 2H), 2.26-2.17 (m, 1H), 1.79-7-1.67 (m, 2H), 0.92-0.90 (m, 6H); ${ }^{13} \mathrm{C}$ $\mathrm{NMR}\left(\mathrm{CDCl}_{3}, 100 \mathrm{MHz}\right): \delta 141.9,139.8,137.29,137.25,131.4,131.0,128.54,128.49$, 126.0, 121.2, 76.0, 37.2, 32.2, 27.8, 23.2. IR (neat) $\mathrm{cm}^{-1}$ ṽ: 3348, 2960, 2927, 2866, 1586, 1454, 1261, 1094, 1072, 1015, 799, 747, 699, 493. HRMS (EI(+), 70 eV): $\mathrm{C}_{20} \mathrm{H}_{23} \mathrm{OBr}$ [M]: calcd: 358.0932, found: 358.0928. HPLC (Chiralcel OD-H column, hexanes: $i-\mathrm{PrOH}=90: 10,1.0 \mathrm{~mL} / \mathrm{min}, 210 \mathrm{~nm}), \mathrm{t}_{\text {minor }}=5.1 \mathrm{~min}, \mathrm{t}_{\text {major }}=6.7 \mathrm{~min}$, ee $=$ $90 \% \cdot[\alpha]_{\mathrm{D}}^{30}=-1.0,\left(\mathrm{c}=0.4, \mathrm{CH}_{2} \mathrm{Cl}_{2}\right)$.

\section{(R,E)-4-(4-fluorophenyl)-6-methyl-1-phenylhept-4-en-3-ol (15d)}<smiles>CC(C)/C=C(/c1ccc(F)cc1)[C@H](O)CCc1ccccc1</smiles>

According to general procedure, $15 \mathbf{d}(40.0 \mathrm{mg}, 0.13 \mathrm{mmol})$ was obtained as colorless oil in $67 \%$ yield after chromatography (silica gel, eluent petroleum ether /ethyl acetate $=15 / 1$ to $5 / 1) .{ }^{1} \mathrm{H}$ NMR $\left(\mathrm{CDCl}_{3}, 400 \mathrm{MHz}\right) \delta$ 7.27-7.24 (m, 2H), 7.19-7.10 (m, 5H), 7.05-7.01 (m, 2H), $5.50(\mathrm{~d}, J=8.0 \mathrm{~Hz}, 1 \mathrm{H}), 4.27-4.21(\mathrm{~m}, 1 \mathrm{H}), 2.76-2.59(\mathrm{~m}, 2 \mathrm{H}), 2.26-$ $2.17(\mathrm{~m}, 1 \mathrm{H}), 1.80-1.66(\mathrm{~m}, 2 \mathrm{H}), 0.92-0.91(\mathrm{~m}, 6 \mathrm{H}) ;{ }^{13} \mathrm{C} \mathrm{NMR}\left(\mathrm{CDCl}_{3}, 100 \mathrm{MHz}\right): \delta$ $162.1(\mathrm{~d}, J=244 \mathrm{~Hz}), 142.0,139.9,137.2,134.2,130.9(\mathrm{~d}, J=7.7 \mathrm{~Hz}), 128.6$ (d, $J=$ $6.0 \mathrm{~Hz}), 126.0,115.2(\mathrm{~d}, J=21.0 \mathrm{~Hz}), 76.2,37.2,32.2,27.8,23.2 ;{ }^{19} \mathrm{~F}$ NMR (376 MHz, $\left.\mathrm{CDCl}_{3}\right) \delta-115.69-115.76(\mathrm{~m}, 1 \mathrm{~F})$. IR (neat) $\mathrm{cm}^{-1} \tilde{\mathrm{v}}: 3357,2956,2928,2866,1602$, 1507, 1454, 1361, 1221, 1157, 1092, 1052, 1028, 1015, 918, 839, 816, 774, 747, 698, 545, 512. HRMS (EI(+), $70 \mathrm{eV}): \mathrm{C}_{20} \mathrm{H}_{23} \mathrm{OF}$ [M]: calcd: 298.1733, found: 298.1740. HPLC (Chiralcel OD-H column, hexanes: $i-\mathrm{PrOH}=90: 10,1.0 \mathrm{~mL} / \mathrm{min}, 210 \mathrm{~nm}$ ), $\mathrm{t}_{\text {minor }}$ 
$=4.8 \mathrm{~min}, \mathrm{t}_{\text {major }}=6.3 \mathrm{~min}, \mathrm{ee}=96 \% .[\alpha]_{\mathrm{D}}^{30}=12.3,\left(\mathrm{c}=0.2, \mathrm{CH}_{2} \mathrm{Cl}_{2}\right)$.

(R,Z)-4-methyl-1-phenyl-2-(thiophen-2-yl)pent-2-en-1-ol (16d)

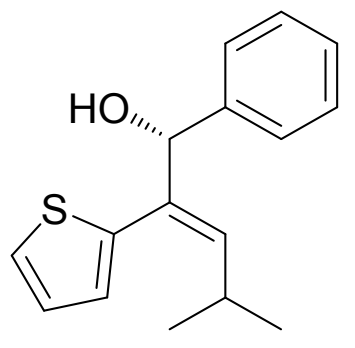

According to general procedure, 16d $(38.2 \mathrm{mg}, 0.15 \mathrm{mmol})$ was obtained as colorless oil in $74 \%$ yield after chromatography (silica gel, eluent petroleum ether /ethyl acetate $=10 / 1$ to $3 / 1) .{ }^{1} \mathrm{H}$ NMR $\left(\mathrm{CDCl}_{3}, 400 \mathrm{MHz}\right) \delta 7.35-7.27(\mathrm{~m}, 5 \mathrm{H}), 7.19-7.17(\mathrm{~m}, 1 \mathrm{H})$, 6.92-6.89 (m,1H), 6.67-6.66 (m, 1H), $5.82(\mathrm{~d}, J=8.0 \mathrm{~Hz}, 1 \mathrm{H}), 5.40(\mathrm{~d}, J=4.0 \mathrm{~Hz}, 1 \mathrm{H})$, 2.68-2.57 (m, 1H), $1.03(\mathrm{dd}, J=8.0 \mathrm{~Hz}, 6 \mathrm{H}) ;{ }^{13} \mathrm{C} \mathrm{NMR}\left(\mathrm{CDCl}_{3}, 100 \mathrm{MHz}\right): \delta 142.0$, 140.0, 138.1, 133.1, 128.4, 127.7, 127.0, 126.7, 126.6, 125.4, 78.5, 28.2, 23.2. IR (neat) $\mathrm{cm}^{-1} \tilde{\mathrm{v}}: 3381,2958,2927,2865,1492,1451,1382,1362,1191,1081,1034,833,696$, 616, 536. HRMS (EI(+), $70 \mathrm{eV}): \mathrm{C}_{16} \mathrm{H}_{18} \mathrm{OS}$ [M]: calcd: 258.1078, found: 258.1084. HPLC (Chiralcel OD-H column, hexanes: $i-\mathrm{PrOH}=98: 2,0.8 \mathrm{~mL} / \mathrm{min}, 210 \mathrm{~nm}$ ), $\mathrm{t}_{\text {minor }}=$ $11.9 \mathrm{~min}, \mathrm{t}_{\text {major }}=12.6 \mathrm{~min}$, ee $=90 \% .[\alpha]_{\mathrm{D}}{ }^{30}=-12.4,\left(\mathrm{c}=0.4, \mathrm{CH}_{2} \mathrm{Cl}_{2}\right)$.

\section{(R,Z)-6-methyl-1-phenyl-4-(trimethylsilyl)hept-4-en-3-ol (17d)}

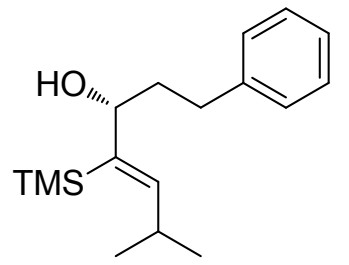

According to general procedure, $\mathbf{1 7 d}(28.8 \mathrm{mg}, 0.10 \mathrm{mmol})$ was obtained as colorless oil in 52\% yield after chromatography (silica gel, eluent petroleum ether /ethyl acetate $=15 / 1$ to $10 / 1) .{ }^{1} \mathrm{H} \mathrm{NMR}\left(\mathrm{CDCl}_{3}, 400 \mathrm{MHz}\right) \delta 7.30-7.26(\mathrm{~m}, 2 \mathrm{H}), 7.20-7.16(\mathrm{~m}, 3 \mathrm{H})$, $6.00(\mathrm{~d}, J=12.0 \mathrm{~Hz}, 1 \mathrm{H}), 4.19-4.16(\mathrm{~m}, 1 \mathrm{H}), 2.79-2.56(\mathrm{~m}, 3 \mathrm{H}), 1.87-1.73(\mathrm{~m}, 2 \mathrm{H})$, $0.97(\mathrm{~d}, J=4.0 \mathrm{~Hz}, 1 \mathrm{H}), 0.15(\mathrm{~s}, 9 \mathrm{H}) ;{ }^{13} \mathrm{C} \mathrm{NMR}\left(\mathrm{CDCl}_{3}, 100 \mathrm{MHz}\right): \delta 149.3,142.4$, 139.5, 128.6, 128.5, 125.9, 75.9, 39.8, 32.7, 30.9, 23.1, 1.0. IR (neat) $\mathrm{cm}^{-1} \tilde{\mathrm{v}}: 3413,2958$, 2925, 2864, 1608, 1495, 1454, 1361, 1249, 1089, 1055, 867, 750, 698, 628, 490. HRMS (EI(+), $70 \mathrm{eV}$ ): $\mathrm{C}_{17} \mathrm{H}_{28} \mathrm{OSi}[\mathrm{M}$ ]: calcd: 276.1909, found: 276.1912. HPLC (Chiralcel OD-H column, hexanes: $i$-PrOH $=98: 2,1.0 \mathrm{~mL} / \mathrm{min}, 210 \mathrm{~nm}), \mathrm{t}_{\text {minor }}=5.5 \mathrm{~min}$, $\mathrm{t}_{\text {major }}=$ $6.1 \mathrm{~min}$, ee $=94 \% .[\alpha]_{\mathrm{D}}^{30}=14.2,\left(\mathrm{c}=0.3, \mathrm{CH}_{2} \mathrm{Cl}_{2}\right)$. 


\section{(R,E)-1-cyclohexyl-2,5-diphenylpent-1-en-3-ol (18d)}<smiles>O[C@H](CCc1ccccc1)/C(=C/C1CCCCC1)c1ccccc1</smiles>

According to general procedure, $\mathbf{1 8 d}(39.1 \mathrm{mg}, 0.12 \mathrm{mmol})$ was obtained as colorless oil in $61 \%$ yield after chromatography (silica gel, eluent petroleum ether /ethyl acetate $=15 / 1$ to $10 / 1) .{ }^{1} \mathrm{H} \mathrm{NMR}\left(\mathrm{CDCl}_{3}, 400 \mathrm{MHz}\right) \delta$ 7.36-7.23 (m, 5H), 7.18-7.12 (m, 5H), $5.51(\mathrm{~d}, J=12.0 \mathrm{~Hz}, 1 \mathrm{H}), 4.29-4.26(\mathrm{~m}, 1 \mathrm{H}), 2.77-2.59(\mathrm{~m}, 2 \mathrm{H}), 1.95-1.89(\mathrm{~m}, 1 \mathrm{H})$, 1.82-1.55 (m, 8H), 1.06-1.01 (m, 5H); $\left.{ }^{13} \mathrm{C} \mathrm{NMR} \mathrm{(} \mathrm{CDCl}_{3}, 100 \mathrm{MHz}\right): \delta 142.1,141.3$, $138.5,135.2,129.3,128.6,128.4,128.2$, 127.0, 125.9, 76.3, 37.3, 33.43, 33.38, 32.2, 26.02, 25.7. IR (neat) $\mathrm{cm}^{-1} \tilde{\mathrm{v}}: 3356,2922,2849,1601,1494,1447,1378,1261,1071$, 1029, 951, 900, 746, 700, 500. HRMS (EI(+), $70 \mathrm{eV}): \mathrm{C}_{23} \mathrm{H}_{28} \mathrm{O}[\mathrm{M}]:$ calcd: 320.2140, found: 320.2144. HPLC (Chiralcel OD-H column, hexanes: $i$-PrOH $=90: 10,1.0$ $\mathrm{mL} / \mathrm{min}, 210 \mathrm{~nm}), \mathrm{t}_{\text {minor }}=4.8 \mathrm{~min}, \mathrm{t}_{\text {major }}=6.3 \mathrm{~min}$, ee $=95 \% .[\alpha]_{\mathrm{D}}{ }^{30}=2.6,(\mathrm{c}=0.3$, $\mathrm{CH}_{2} \mathrm{Cl}_{2}$ ).

\section{(R,E)-3-cyclohexyl-1,2-diphenylprop-2-en-1-ol (19d)}<smiles>O[C@H](/C(=C/C1CCCCC1)c1ccccc1)c1ccccc1</smiles>

According to general procedure, $19 \mathrm{~d}(31.6 \mathrm{mg}, 0.11 \mathrm{mmol})$ was obtained as colorless oil in 54\% yield after chromatography (silica gel, eluent petroleum ether /ethyl acetate $=15 / 1$ to $5 / 1) .{ }^{1} \mathrm{H}$ NMR $\left(\mathrm{CDCl}_{3}, 400 \mathrm{MHz}\right) \delta$ 7.34-7.20 (m, 8H), 6.90-6.88 (m, 2H), $5.69(\mathrm{~d}, J=8.0 \mathrm{~Hz}, 1 \mathrm{H}), 5.39-5.36(\mathrm{~m}, 1 \mathrm{H}), 2.01-1.96(\mathrm{~m}, 1 \mathrm{H}), 1.63-1.56(\mathrm{~m}, 6 \mathrm{H}), 1.17-$ $1.11(\mathrm{~m}, 5 \mathrm{H}) ;{ }^{13} \mathrm{C} \mathrm{NMR}\left(\mathrm{CDCl}_{3}, 100 \mathrm{MHz}\right): \delta 142.3,141.1,138.2,135.2,129.4,128.2$, 128.0, 127.5, 127.0, 126.8, 78.7, 37.4, 33.4, 26.1, 25.7. IR (neat) $\mathrm{cm}^{-1} \tilde{\mathrm{v}}: 3393,2922$, 2850, 1493, 1448, 1261, 1064, 1024, 967, 802, 759, 727, 701, 602. HRMS (EI(+), 70 eV): $\mathrm{C}_{21} \mathrm{H}_{24} \mathrm{O}[\mathrm{M}]$ : calcd: 292.1827, found: 292.1823. HPLC (Chiralcel OD-H column, 
hexanes: $i-\mathrm{PrOH}=90: 10,1.0 \mathrm{~mL} / \mathrm{min}, 210 \mathrm{~nm}), \mathrm{t}_{\text {minor }}=4.3 \mathrm{~min}, \mathrm{t}_{\text {major }}=4.7 \mathrm{~min}$, ee $=$ $96 \% .[\alpha]_{\mathrm{D}}{ }^{30}=-22.3,\left(\mathrm{c}=0.8, \mathrm{CH}_{2} \mathrm{Cl}_{2}\right)$.

(R,E)-2,5-diphenyl-1-(tetrahydro-2H-pyran-4-yl)pent-1-en-3-ol (20d)<smiles>O[C@H](CCc1ccccc1)/C(=C/C1CCOCC1)c1ccccc1</smiles>

According to general procedure, $20 \mathrm{~d}(40.6 \mathrm{mg}, 0.13 \mathrm{mmol})$ was obtained as colorless oil in 63\% yield after chromatography (silica gel, eluent petroleum ether /ethyl acetate $=15 / 1$ to $5 / 1) .{ }^{1} \mathrm{H}$ NMR $\left(\mathrm{CDCl}_{3}, 400 \mathrm{MHz}\right) \delta$ 7.39-7.24 (m, 5H), 7.19-7.13 (m, 5H), $5.53(\mathrm{~d}, J=8.0 \mathrm{~Hz}, 1 \mathrm{H}), 4.31-4.28(\mathrm{~m}, 1 \mathrm{H}), 3.89-3.86(\mathrm{~m}, 2 \mathrm{H}), 3.28-3.21(\mathrm{~m}, 2 \mathrm{H}), 2.79$ $2.61(\mathrm{~m}, 2 \mathrm{H}), 2.23-2.13(\mathrm{~m}, 1 \mathrm{H}), 1.84-1.66(\mathrm{~m}, 4 \mathrm{H}), 1.56-1.43(\mathrm{~m}, 4 \mathrm{H}) ;{ }^{13} \mathrm{C} \mathrm{NMR}$ $\left(\mathrm{CDCl}_{3}, 100 \mathrm{MHz}\right): \delta 142.9,142.0,138.2,132.9,129.1,128.54,128.46,128.4,127.3$, 125.9, 76.0, 67.4, 37.3, 34.6, 32.9, 32.1. IR (neat) $\mathrm{cm}^{-1} \tilde{\mathrm{v}}: 3412,2843,2757,1600,1493$, 1453, 1441, 1386, 1262, 1173, 1155, 1080, 1012, 985, 917, 868, 812, 747, 700, 576, 486. HRMS (EI(+), $70 \mathrm{eV}): \mathrm{C}_{22} \mathrm{H}_{26} \mathrm{O}_{2}$ [M]: calcd: 322.1933, found: 322.1930. HPLC (Chiralcel OD-H column, hexanes: $i-\mathrm{PrOH}=90: 10,1.0 \mathrm{~mL} / \mathrm{min}, 210 \mathrm{~nm}$ ), $\mathrm{t}_{\text {minor }}=8.2$ $\min , \mathrm{t}_{\text {major }}=12.7 \mathrm{~min}$, ee $=93 \% .[\alpha]_{\mathrm{D}}{ }^{30}=-5.9,\left(\mathrm{c}=1.2, \mathrm{CH}_{2} \mathrm{Cl}_{2}\right)$.

\section{(R,E)-5-((tert-butyldimethylsilyl)oxy)-4,4-difluoro-1,2-diphenylpent-2-en-1-ol} (21d)<smiles>CC(C)(C)[Si](C)(C)OCC(F)(F)/C=C(\c1ccccc1)[C@H](O)c1ccccc1</smiles>

According to general procedure, 21d $(43.7 \mathrm{mg}, 0.11 \mathrm{mmol})$ was obtained as colorless oil in 54\% yield after chromatography (silica gel, eluent petroleum ether /ethyl acetate $=10 / 1$ to $3 / 1) .{ }^{1} \mathrm{H} \mathrm{NMR}\left(\mathrm{CDCl}_{3}, 400 \mathrm{MHz}\right) \delta 7.28-7.14(\mathrm{~m}, 8 \mathrm{H}), 6.89-6.87(\mathrm{~m}, 2 \mathrm{H})$, $6.17(\mathrm{t}, J=12.0 \mathrm{~Hz}, 1 \mathrm{H}), 5.34-5.30(\mathrm{~m}, 1 \mathrm{H}), 3.54(\mathrm{t}, J=12.0 \mathrm{~Hz}, 1 \mathrm{H}), 2.12(\mathrm{~d}, J=4.0$ $\mathrm{Hz}, 1 \mathrm{H}), 0.85(\mathrm{~s}, 9 \mathrm{H}), 0.02(\mathrm{~s}, 6 \mathrm{H}) ;{ }^{13} \mathrm{C} \mathrm{NMR}\left(\mathrm{CDCl}_{3}, 100 \mathrm{MHz}\right): \delta 150.6(\mathrm{t}, J=7.2$ 
$\mathrm{Hz}), 140.4,136.3,128.9$ (t, $J=1.8 \mathrm{~Hz}), 128.6,128.3,127.7,127.6,127.2,126.1,120.5$ $(\mathrm{t}, J=26.7 \mathrm{~Hz}), 120.0(\mathrm{t}, J=239 \mathrm{~Hz}), 78.5,65.7(\mathrm{t}, J=33 \mathrm{~Hz}), 25.9,18.5,-5.4 ;{ }^{19} \mathrm{~F}$ NMR (376 MHz, $\left.\mathrm{CDCl}_{3}\right) \delta-96.83-97.00$ (m, 2F). IR (neat) $\mathrm{cm}^{-1} \tilde{\mathrm{v}}: 3375,2955,2929$, 2857, 1667, 1493, 1459, 1387, 1290, 1255, 1120, 1063, 944, 925, 836, 778, 699, 592, 542. HRMS (ESI+): $\mathrm{C}_{23} \mathrm{H}_{34} \mathrm{O}_{2} \mathrm{NF}_{2} \mathrm{Si}\left[\mathrm{M}+\mathrm{NH}_{4}\right]^{+}$calcd: 422.2321 , found: 422.2318 . HPLC (Chiralcel OD-H column, hexanes: $i-\mathrm{PrOH}=95: 5,1.0 \mathrm{~mL} / \mathrm{min}, 210 \mathrm{~nm}$ ), $\mathrm{t}_{\text {minor }}=$ $5.4 \mathrm{~min}, \mathrm{t}_{\text {major }}=6.0 \mathrm{~min}$, ee $=86 \% .[\alpha]_{\mathrm{D}}{ }^{30}=-2.3,\left(\mathrm{c}=0.5, \mathrm{CH}_{2} \mathrm{Cl}_{2}\right)$.

\section{(R,E)-7-((tert-butyldimethylsilyl)oxy)-6,6-difluoro-1,4-diphenylhept-4-en-3-ol} (22d)<smiles>CC(C)(C)[Si](C)(C)OCC(F)(F)/C=C(\c1ccccc1)[C@@H](O)CCc1ccccc1</smiles>

According to general procedure, $22 \mathrm{~d}(51.0 \mathrm{mg}, 0.12 \mathrm{mmol})$ was obtained as colorless oil in 59\% yield after chromatography (silica gel, eluent petroleum ether /ethyl acetate $=10 / 1$ to $3 / 1) .{ }^{1} \mathrm{H}$ NMR $\left(\mathrm{CDCl}_{3}, 400 \mathrm{MHz}\right) \delta 7.32-7.30(\mathrm{~m}, 3 \mathrm{H}), 7.25-7.22(\mathrm{~m}, 2 \mathrm{H})$, 7.17-7.15 (m, 3H), 7.11-7.09 (m, 2H), $5.94(\mathrm{t}, J=12.0,1 \mathrm{H}), 4.32(\mathrm{~m}, 1 \mathrm{H}), 3.55(\mathrm{t}, J=$ 12.0, 2H), 2.81-2.74 (m, 1H), 2.69-2.61 (m, 1H), 1.84-1.78 (m, 1H), 1.72-1.67 (m, 1H), $0.85(\mathrm{~s}, 1 \mathrm{H}), 0.01(\mathrm{~s}, 1 \mathrm{H}) ;{ }^{13} \mathrm{C} \mathrm{NMR}\left(\mathrm{CDCl}_{3}, 100 \mathrm{MHz}\right): \delta 151.6(\mathrm{t}, J=6.9 \mathrm{~Hz}), 141.6$, 136.4, 128.6 (t, $J=1.9 \mathrm{~Hz}), 128.5,128.5,128.0,128.0,126.1,120.5$ (t, $J=26.6 \mathrm{~Hz}$ ), $119.8(\mathrm{t}, J=239.6 \mathrm{~Hz}), 75.6,65.7(\mathrm{t}, J=32.6 \mathrm{~Hz}), 36.5,31.7,25.9,18.5,-5.3 ;{ }^{19} \mathrm{~F} \mathrm{NMR}$ $\left(376 \mathrm{MHz}, \mathrm{CDCl}_{3}\right) \delta-96.91$ (dq, $\left.J=15.04,7.52 \mathrm{~Hz}, 2 \mathrm{~F}\right) . \mathrm{IR}$ (neat) $\mathrm{cm}^{-1} \tilde{\mathrm{v}}: 3415,2955$, 2930, 2858, 1667, 1603, 1494, 1459, 1392, 1259, 1093, 1018, 926, 838, 797, 748, 701, 526. HRMS (ESI+): $\mathrm{C}_{25} \mathrm{H}_{38} \mathrm{O}_{2} \mathrm{NF}_{2} \mathrm{Si}\left[\mathrm{M}+\mathrm{NH}_{4}\right]^{+}$calcd: 450.2634 , found: 450.2631 . HPLC (Chiralcel OD-H column, hexanes: $i-\mathrm{PrOH}=90: 10,1.0 \mathrm{~mL} / \mathrm{min}, 210 \mathrm{~nm}$ ), $\mathrm{t}_{\text {minor }}$ $=4.4 \mathrm{~min}, \mathrm{t}_{\text {major }}=5.9 \mathrm{~min}$, ee $=95 \% .[\alpha]_{\mathrm{D}}{ }^{30}=-6.8,\left(\mathrm{c}=0.3, \mathrm{CH}_{2} \mathrm{Cl}_{2}\right)$.

(R,E)-1-((tert-butyldimethylsilyl)oxy)-2,2-difluoro-4-phenyldec-3-en-5-ol (23d)

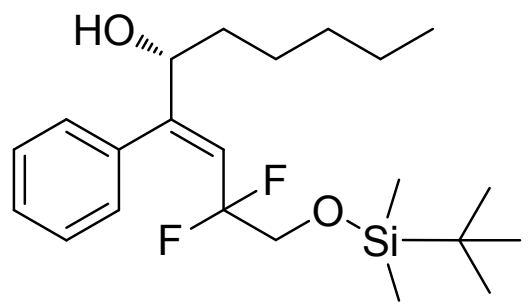


According to general procedure, 23d $(49.4 \mathrm{mg}, 0.12 \mathrm{mmol})$ was obtained as colorless oil in $62 \%$ yield after chromatography (silica gel, eluent petroleum ether /ethyl acetate $=10 / 1$ to $3 / 1) .{ }^{1} \mathrm{H}$ NMR $\left(\mathrm{CDCl}_{3}, 400 \mathrm{MHz}\right) \delta$ 7.35-7.32 (m, 3H), 7.18-7.16 (m, 2H), $5.92(\mathrm{t}, J=12.0 \mathrm{~Hz}, 1 \mathrm{H}), 4.34-4.26(\mathrm{~m}, 1 \mathrm{H}), 3.56(\mathrm{t}, J=12.0 \mathrm{~Hz}, 2 \mathrm{H}), 1.69$ (d, $J=4.0$ $\mathrm{Hz}, 1 \mathrm{H}), 1.50-1.21(\mathrm{~m}, 8 \mathrm{H}), 0.87-0.84(\mathrm{~m}, 12 \mathrm{H}), 0.01(\mathrm{~s}, 6 \mathrm{H}) ;{ }^{13} \mathrm{C} \mathrm{NMR}\left(\mathrm{CDCl}_{3}, 100\right.$ MHz): $\delta 151.8(\mathrm{t}, J=7.1 \mathrm{~Hz}), 136.6,128.7$ (t, $J=1.9 \mathrm{~Hz}), 127.9,127.8,120.3(\mathrm{t}, J=$ $26.5 \mathrm{~Hz}), 119.9$ (t, $J=239.6 \mathrm{~Hz}), 76.3,65.7$ (t, $J=32.5 \mathrm{~Hz}), 35.1,31.7,25.9,25.1$, 22.7, 18.5, 14.2, -5.4; ${ }^{19} \mathrm{~F}$ NMR (376 MHz, $\left.\mathrm{CDCl}_{3}\right) \delta-96.90$ (q, $\left.J=15.04 \mathrm{~Hz}, 2 \mathrm{~F}\right)$. IR (neat) $\mathrm{cm}^{-1} \tilde{\mathrm{v}}: 3389,2956,2930,2858,1667,1493,1464,1293,1259,1119,1021,927$, 838, 798, 782, 703, 583. HRMS (ESI +$): \mathrm{C}_{22} \mathrm{H}_{40} \mathrm{O}_{2} \mathrm{NF}_{2} \mathrm{Si}\left[\mathrm{M}+\mathrm{NH}_{4}\right]^{+}$calcd: 416.2791, found: 416.2788. HPLC (Chiralcel OD-H column, hexanes: $i-\mathrm{PrOH}=95: 5,1.0 \mathrm{~mL} / \mathrm{min}$, $210 \mathrm{~nm}), \mathrm{t}_{\text {minor }}=3.7 \mathrm{~min}, \mathrm{t}_{\text {major }}=4.0 \mathrm{~min}$, ee $=96 \% .[\alpha]_{\mathrm{D}}{ }^{30}=-11.7,\left(\mathrm{c}=0.6, \mathrm{CH}_{2} \mathrm{Cl}_{2}\right)$.

(R,E)-7-(benzyloxy)-6,6-difluoro-1,4-diphenylhept-4-en-3-ol (24d)<smiles>O[C@H](CCc1ccccc1)/C(=C/C(F)(F)COCc1ccccc1)c1ccccc1</smiles>

According to general procedure, $\mathbf{2 4 d}(44.9 \mathrm{mg}, 0.11 \mathrm{mmol})$ was obtained as colorless oil in 55\% yield after chromatography (silica gel, eluent petroleum ether /ethyl acetate $=10 / 1$ to $5 / 1) .{ }^{1} \mathrm{H}$ NMR $(\mathrm{CDCl} 3,400 \mathrm{MHz}) \delta$ 7.34-7.23 (m, 9H), 7.18-7.10 (m, 4H), 6.99-6.97 (m, 2H), $6.05(\mathrm{t}, J=12.0 \mathrm{~Hz}, 1 \mathrm{H}), 4.51(\mathrm{~s}, 2 \mathrm{H}), 4.36-4.30(\mathrm{~m}, 1 \mathrm{H}), 3.52(\mathrm{t}, J$ $=12.0 \mathrm{~Hz}, 2 \mathrm{H}), 2.78-2.61(\mathrm{~m}, 2 \mathrm{H}), 2.11(\mathrm{~s}, 1 \mathrm{H}), 1.87-1.64(\mathrm{~m}, 2 \mathrm{H}) ;{ }^{13} \mathrm{C} \mathrm{NMR}\left(\mathrm{CDCl}_{3}\right.$, $100 \mathrm{MHz}): \delta 145.5(\mathrm{t}, J=8.0 \mathrm{~Hz}), 141.5,137.4, \quad 135.5,128.6(\mathrm{t}, J=8.0 \mathrm{~Hz})$, $128.6,128.6,128.5,128.1,128.0,126.9,126.8,126.1,122.9,119.6(\mathrm{t}, J=$ $240.0 \mathrm{~Hz}), 75.8,74.0,71.0(\mathrm{t}, J=8.0 \mathrm{~Hz}), 36.9,31.7$; ${ }^{19} \mathrm{~F}$ NMR $\left(376 \mathrm{MHz}, \mathrm{CDCl}_{3}\right)$ $\delta-93.00$ (q, $J=15.04 \mathrm{~Hz}, 2 \mathrm{~F}$ ). IR (neat) $\mathrm{cm}^{-1} \tilde{\mathrm{v}}: 3424,3028,2924,2860,1667,1602$, 1494, 1453, 1366, 1260, 1093, 1071, 1026, 926, 800, 746, 699, 598, 508. HRMS (ESI+): $\mathrm{C}_{26} \mathrm{H}_{30} \mathrm{O}_{2} \mathrm{NF}_{2}\left[\mathrm{M}+\mathrm{NH}_{4}\right]^{+}$calcd: 426.2239, found: 426.2236. HPLC (Chiralcel IE-H column, hexanes: $i$-PrOH $=95: 5,1.0 \mathrm{~mL} / \mathrm{min}, 210 \mathrm{~nm}), \mathrm{t}_{\text {minor }}=7.8 \mathrm{~min}, \mathrm{t}_{\text {major }}=$ $8.2 \min$, ee $=94 \% .[\alpha]_{\mathrm{D}}{ }^{30}=-21.3,\left(\mathrm{c}=0.9, \mathrm{CH}_{2} \mathrm{Cl}_{2}\right)$.

(R,E)-1-(benzyloxy)-2,2-difluoro-4-phenyldec-3-en-5-ol (25d) 
<smiles>CCCCC[C@H](O)/C(=C/C(F)(F)COCc1ccccc1)c1ccccc1</smiles>

According to general procedure, $\mathbf{2 5 d}(44.9 \mathrm{mg}, 0.12 \mathrm{mmol})$ was obtained as colorless oil in $60 \%$ yield after chromatography (silica gel, eluent petroleum ether /ethyl acetate $=10 / 1$ to 5/1). ${ }^{1} \mathrm{H} \mathrm{NMR}\left(\mathrm{CDCl}_{3}, 400 \mathrm{MHz}\right) \delta 7.36-7.26(\mathrm{~m}, 8 \mathrm{H}), 7.14-7.12(\mathrm{~m}, 2 \mathrm{H})$, $5.96(\mathrm{t}, J=12.0 \mathrm{~Hz}, 1 \mathrm{H}), 4.48(\mathrm{~s}, 2 \mathrm{H}), 4.32-4.26(\mathrm{~m}, 1 \mathrm{H}), 3.48-3.34(\mathrm{~m}, 2 \mathrm{H}), 1.81$ $(\mathrm{s}, 1 \mathrm{H}), 1.48-1.18(\mathrm{~m}, 9 \mathrm{H}), 0.85(\mathrm{t}, J=8.0 \mathrm{~Hz}, 3 \mathrm{H}) ;{ }^{13} \mathrm{C} \mathrm{NMR}\left(\mathrm{CDCl}_{3}, 100 \mathrm{MHz}\right): \delta$ $152.5(\mathrm{t}, J=6.1 \mathrm{~Hz}), 137.4,136.5,128.7(\mathrm{t}, J=1.8 \mathrm{~Hz}), 128.6,128.0,128.0,127.9$, 127.9, $120.1(\mathrm{t}, J=26.9 \mathrm{~Hz}), 119.8(\mathrm{t}, J=239.2 \mathrm{~Hz}), 76.3,73.8,71.4(\mathrm{t}, J=32.0 \mathrm{~Hz})$, 35.0, 31.7, 25.1, 22.7, 14.2. ${ }^{19} \mathrm{~F}$ NMR $\left(376 \mathrm{MHz} \mathrm{CDCl}_{3}\right) \delta-93.60$ (q, $J=15.04 \mathrm{~Hz}$, 2F). IR (neat) $\mathrm{cm}^{-1} \tilde{\mathrm{v}}$ : 3427, 2928, 2859, 1666, 1494, 1454, 1366, 1289, 1260, 1194 , 1101, 1073, 1028, 943, 736, 699, 595, 540. HRMS (ESI+): $\mathrm{C}_{23} \mathrm{H}_{32} \mathrm{O}_{2} \mathrm{NF}_{2}\left[\mathrm{M}+\mathrm{NH}_{4}\right]^{+}$ calcd: 392.2396, found: 392.2393. HPLC (Chiralcel OD-H column, hexanes: $i-\mathrm{PrOH}=$ $90: 10,1.0 \mathrm{~mL} / \mathrm{min}, 210 \mathrm{~nm}), \mathrm{t}_{\text {minor }}=4.5 \mathrm{~min}, \mathrm{t}_{\text {major }}=5.5 \mathrm{~min}$, ee $=95 \% .[\alpha]_{\mathrm{D}}{ }^{30}=-17.6$, (c $=0.9, \mathrm{CH}_{2} \mathrm{Cl}_{2}$ ).

\section{(R,Z)-6-methyl-1-phenyl-4-(thiophen-2-yl)hept-4-en-3-ol (26d)}<smiles>O[C@H](CCc1ccccc1)/C(=C/C(F)(F)COCc1ccccc1)c1cccs1</smiles>

According to general procedure, 26d $(58.0 \mathrm{mg}, 0.14 \mathrm{mmol})$ was obtained as colorless oil in $70 \%$ yield after chromatography (silica gel, eluent petroleum ether /ethyl acetate $=10 / 1$ to $3 / 1) .{ }^{1} \mathrm{H} \mathrm{NMR}\left(\mathrm{CDCl}_{3}, 400 \mathrm{MHz}\right) \delta 7.37-7.23(\mathrm{~m}, 8 \mathrm{H}), 7.19-7.11(\mathrm{~m}, 3 \mathrm{H})$, 7.00-6.98 (m, 2H), $6.06(\mathrm{t}, J=12.0 \mathrm{~Hz}, 1 \mathrm{H}), 4.52(\mathrm{~s}, 2 \mathrm{H}), 4.38-4.32(\mathrm{~m}, 1 \mathrm{H}), 3.52(\mathrm{t}, J$ $=12.0 \mathrm{~Hz}, 1 \mathrm{H}), 2.78-2.62(\mathrm{~m}, 2 \mathrm{H}), 1.97-1.91(\mathrm{~m}, 1 \mathrm{H}), 1.88-1.70(\mathrm{~m}, 2 \mathrm{H}) ;{ }^{13} \mathrm{C} \mathrm{NMR}$ $\left(\mathrm{CDCl}_{3}, 100 \mathrm{MHz}\right): \delta 145.5(\mathrm{t}, J=7.8 \mathrm{~Hz}), 141.5,137.4,135.5,128.6(\mathrm{t}, J=2.9 \mathrm{~Hz})$, 128.6, 128.6, 128.5, 128.1, 128.0, 126.9, 126.8, 126.1, 122.9 (t, $J=26.8 \mathrm{~Hz}), 119.6$ (t, $J=239.9 \mathrm{~Hz}), 75.8,74.0,71.0(\mathrm{t}, J=31.4 \mathrm{~Hz}), 36.9,31.7 ;{ }^{19} \mathrm{~F} \mathrm{NMR}\left(376 \mathrm{MHz}, \mathrm{CDCl}_{3}\right)$ $\delta-93.00$ (q, $J=15.04 \mathrm{~Hz}, 2 \mathrm{~F}$ ). IR (neat) $\mathrm{cm}^{-1} \tilde{\mathrm{v}}: 3429,2923,2861,1658,1495,1453$, $1288,1261,1094,1071,941,849,803,745,698,597,488$. HRMS (ESI+): $\mathrm{C}_{24} \mathrm{H}_{25} \mathrm{O}_{2} \mathrm{~F}_{2} \mathrm{~S}$ $[\mathrm{M}+\mathrm{H}]^{+}$calcd: 415.1538 , found: 415.1535. HPLC (Chiralcel OD-H column, hexanes: $i-$ $\mathrm{PrOH}=90: 10,1.0 \mathrm{~mL} / \mathrm{min}, 210 \mathrm{~nm}), \mathrm{t}_{\text {minor }}=8.8 \mathrm{~min}, \mathrm{t}_{\text {major }}=14.4 \mathrm{~min}$, ee $=95 \% .[\alpha]_{\mathrm{D}}{ }^{30}$ 
$=-30.5,\left(\mathrm{c}=2.3, \mathrm{CH}_{2} \mathrm{Cl}_{2}\right)$.

(R,E)-7-(benzyloxy)-6,6-difluoro-1-phenyl-4-(p-tolyl)hept-4-en-3-ol (27d)

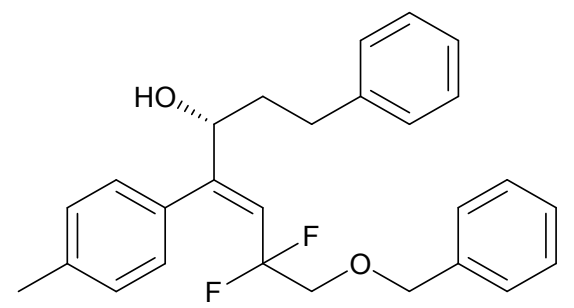

According to general procedure, $\mathbf{2 7 d}(54.1 \mathrm{mg}, 0.13 \mathrm{mmol})$ was obtained as colorless oil in 64\% yield after chromatography (silica gel, eluent petroleum ether /ethyl acetate $=10 / 1$ to 5/1). ${ }^{1} \mathrm{H}$ NMR $\left(\mathrm{CDCl}_{3}, 400 \mathrm{MHz}\right) \delta$ 7.34-7.14 (m, 8H), 7.11-7.09 (m, 2H), 7.02-7.00 (m, 2H), $5.96(\mathrm{t}, J=12.0 \mathrm{~Hz}, 1 \mathrm{H}), 4.48(\mathrm{~s}, 2 \mathrm{H}), 4.34-4.28(\mathrm{~m}, 1 \mathrm{H}), 3.45-3.37$ $(\mathrm{m}, 2 \mathrm{H}), 2.81-2.60(\mathrm{~m}, 2 \mathrm{H}), 2.35(\mathrm{~s}, 3 \mathrm{H}), 1.86(\mathrm{~s}, 1 \mathrm{H}), 1.85-1.63(\mathrm{~m}, 2 \mathrm{H}) ;{ }^{13} \mathrm{C} \mathrm{NMR}$ $\left(\mathrm{CDCl}_{3}, 100 \mathrm{MHz}\right): \delta 152.3(\mathrm{t}, J=8.0 \mathrm{~Hz}), 141.7,137.8,137.4,133.2,128.7,128.6$, 128.5, 128.5, $128.5(\mathrm{t}, J=8.0 \mathrm{~Hz}), 128.0,128.0,126.0,120.3(\mathrm{t}, J=27.0 \mathrm{~Hz}), 119.9$ (t, $J=238.7 \mathrm{~Hz}), 75.6,73.8,71.3(\mathrm{t}, J=31.8 \mathrm{~Hz}), 36.5,31.7,21.4 ;{ }^{19} \mathrm{~F}$ NMR $(376 \mathrm{MHz}$, $\left.\mathrm{CDCl}_{3}\right) \delta-93.40$ (q, $J=15.04 \mathrm{~Hz}, 2 \mathrm{~F}$ ). IR (neat) $\mathrm{cm}^{-1} \tilde{\mathrm{v}}: 3429,3028,2922,2864,1665$, 1604, 1512, 1496, 1453, 1366, 1290, 1207, 1094, 1071, 1027, 941, 821, 743, 698, 527. HRMS (ESI +$): \mathrm{C}_{27} \mathrm{H}_{32} \mathrm{O}_{2} \mathrm{NF}_{2}\left[\mathrm{M}+\mathrm{NH}_{4}\right]^{+}$calcd: 440.2396, found: 440.2393. HPLC (Chiralcel IG-H column, hexanes: $i-\mathrm{PrOH}=95: 5,0.8 \mathrm{~mL} / \mathrm{min}, 210 \mathrm{~nm}$ ), $\mathrm{t}_{\text {minor }}=10.5$ $\min , \mathrm{t}_{\text {major }}=11.2 \mathrm{~min}$, ee $=94 \% .[\alpha]_{\mathrm{D}}{ }^{30}=0.7,\left(\mathrm{c}=0.3, \mathrm{CH}_{2} \mathrm{Cl}_{2}\right)$.

\section{(R,E)-1-(benzyloxy)-9-chloro-2,2-difluoro-4-(p-tolyl)non-3-en-5-ol (28d)}<smiles>Cc1ccc(/C(=C/C(F)(F)COCc2ccccc2)[C@H](O)CCCCCl)cc1</smiles>

According to general procedure, 28d (43.4 $\mathrm{mg}, 0.11 \mathrm{mmol})$ was obtained as colorless oil in 53\% yield after chromatography (silica gel, eluent petroleum ether /ethyl acetate $=10 / 1$ to $5 / 1) .{ }^{1} \mathrm{H}$ NMR $\left(\mathrm{CDCl}_{3}, 400 \mathrm{MHz}\right) \delta$ 7.37-7.30 (m, 3H), 7.28-7.27 (m, 2H), $7.12(\mathrm{~d}, J=8.0 \mathrm{~Hz}, 1 \mathrm{H}), 7.01(\mathrm{~d}, J=8.0 \mathrm{~Hz}, 1 \mathrm{H}), 5.95(\mathrm{t}, J=12.0 \mathrm{~Hz}, 1 \mathrm{H}), 4.48(\mathrm{~s}$, 2H), 4.32-4.26 (m, 1H), $3.47(\mathrm{t}, J=8.0 \mathrm{~Hz}, 2 \mathrm{H}), 3.42(\mathrm{q}, J=8.0 \mathrm{~Hz}, 2 \mathrm{H}), 2.35(\mathrm{~s}, 3 \mathrm{H})$, 1.74-1.25 (m, 6H); ${ }^{13} \mathrm{C} \mathrm{NMR}\left(\mathrm{CDCl}_{3}, 100 \mathrm{MHz}\right): \delta 152.4(\mathrm{t}, J=7.4 \mathrm{~Hz}), 137.8,137.4$, 133.3, 128.8, 128.6, 128.5 (t, $J=1.9 \mathrm{~Hz}), 128.1,128.0,120.2$ (t, $J=27.0 \mathrm{~Hz}), 119.9$ (t, $J=239 \mathrm{~Hz}), 76.0,73.9,71.3(\mathrm{t}, J=32.1 \mathrm{~Hz}), 44.9,34.1,32.4,22.8,21.4 .{ }^{19} \mathrm{~F}$ NMR $\left(376 \mathrm{MHz}, \mathrm{CDCl}_{3}\right) \delta-93.40$ (q, $\left.J=11.28 \mathrm{~Hz}, 2 \mathrm{~F}\right) . \mathrm{IR}$ (neat) $\mathrm{cm}^{-1} \tilde{\mathrm{v}}: 3428,2923,2867$, 
1722, 1666, 1609, 1512, 1454, 1367, 1260, 1095, 1021, 929, 801, 738, 699, 651, 534. HRMS (ESI+): $\mathrm{C}_{23} \mathrm{H}_{31} \mathrm{O}_{2} \mathrm{NF}_{2} \mathrm{Cl}\left[\mathrm{M}+\mathrm{NH}_{4}\right]^{+}$calcd: 426.2006, found: 426.2001. HPLC (Chiralcel OD-H column, hexanes: $i-\mathrm{PrOH}=90: 10,0.8 \mathrm{~mL} / \mathrm{min}, 210 \mathrm{~nm}$ ), $\mathrm{t}_{\text {minor }}=6.7$ $\min , \mathrm{t}_{\text {major }}=8.4 \mathrm{~min}$, ee $=90 \% .[\alpha]_{\mathrm{D}}^{30}=-11.5,\left(\mathrm{c}=0.9, \mathrm{CH}_{2} \mathrm{Cl}_{2}\right)$.

(R,E)-7,7,7-trifluoro-1,4-diphenylhept-4-en-3-ol (29d)<smiles>O[C@H](CCc1ccccc1)/C(=C/CC(F)(F)F)c1ccccc1</smiles>

According to general procedure, $29 \mathrm{~d}(33.3 \mathrm{mg}, 0.10 \mathrm{mmol})$ was obtained as colorless oil in 52\% yield after chromatography (silica gel, eluent petroleum ether /ethyl acetate $=10 / 1$ to $3 / 1) .{ }^{1} \mathrm{H} \mathrm{NMR}\left(\mathrm{CDCl}_{3}, 400 \mathrm{MHz}\right) \delta 7.40-7.33(\mathrm{~m}, 3 \mathrm{H}), 7.27-7.24(\mathrm{~m}, 3 \mathrm{H})$, 7.19-7.12 (m, 5H), $5.78(\mathrm{~d}, J=8.0 \mathrm{~Hz}, 1 \mathrm{H}), 4.42-4.36(\mathrm{~m}, 1 \mathrm{H}), 2080-2.62(\mathrm{~m} .4 \mathrm{H})$, 1.86-1.76 (m, 2H); ${ }^{13} \mathrm{C} \mathrm{NMR}\left(\mathrm{CDCl}_{3}, 100 \mathrm{MHz}\right): \delta 150.3,141.7,136.7,128.9,128.7$, 128.6, 128.5, 127.9, $126.2(\mathrm{q}, J=275.2 \mathrm{~Hz}) 126.0,115.6(\mathrm{q}, J=3.5 \mathrm{~Hz}), 75.5,37.0$, $33.9(\mathrm{q}, J=29.4 \mathrm{~Hz}), 31.9 .{ }^{19} \mathrm{~F} \mathrm{NMR}\left(376 \mathrm{MHz}, \mathrm{CDCl}_{3}\right) \delta-65.88(\mathrm{t}, J=11.28 \mathrm{~Hz}, 3 \mathrm{~F})$. IR (neat) $\mathrm{cm}^{-1} \tilde{\mathrm{v}}: 3397,3027,2925,2856,1671,1603,1495,1453,1346,1276,1253$, 1139, 1028, 1014, 921, 800, 749, 701. HRMS (EI(+), 70 eV): $\mathrm{C}_{19} \mathrm{H}_{19} \mathrm{OF}_{3}$ [M]: calcd: 320.1388, found: 320.1394. HPLC (Chiralcel OD-H column, hexanes: $i-\mathrm{PrOH}=95: 5$, $1.0 \mathrm{~mL} / \mathrm{min}, 210 \mathrm{~nm}), \mathrm{t}_{\text {minor }}=7.3 \mathrm{~min}, \mathrm{t}_{\text {major }}=11.5 \mathrm{~min}, \mathrm{ee}=92 \% .[\alpha]_{\mathrm{D}}{ }^{30}=7.7,(\mathrm{c}=0.3$, $\mathrm{CH}_{2} \mathrm{Cl}_{2}$ ).

(R,Z)-5,5,5-trifluoro-1-phenyl-2-(thiophen-2-yl)pent-2-en-1-ol (30d)

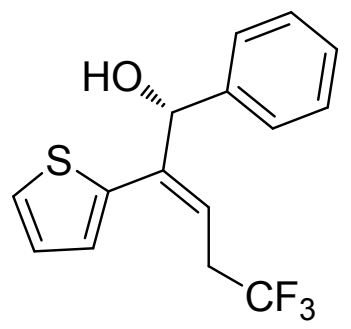

According to general procedure, $\mathbf{3 0 d}(28.0 \mathrm{mg}, 0.10 \mathrm{mmol})$ was obtained as colorless oil in $47 \%$ yield after chromatography (silica gel, eluent petroleum ether /ethyl acetate $=10 / 1$ to $3 / 1)$ afforded the product. ${ }^{1} \mathrm{H}$ NMR $\left(\mathrm{CDCl}_{3}, 400 \mathrm{MHz}\right) \delta 7.32-7.23(\mathrm{~m}, 6 \mathrm{H})$, 6.94-6.92 (m, 1H), 6.71-6.70 (m, 1H), $6.12(\mathrm{t}, J=8.0 \mathrm{~Hz}, 1 \mathrm{H}), 5.46(\mathrm{~s}, 1 \mathrm{H}), 3.06-2.97$ (m, 2H), $2.15(\mathrm{~s}, 1 \mathrm{H}) ;{ }^{13} \mathrm{C} \mathrm{NMR}\left(\mathrm{CDCl}_{3}, 100 \mathrm{MHz}\right): \delta 142.2,141.0,136.5,128.6$, 128.2, 128.0, 127.0, 126.9, 126.5, 126.1 (q, $J=275.2 \mathrm{~Hz}), 118.5$ (q, $J=3.5 \mathrm{~Hz}), 78.2$, 
$34.2(\mathrm{q}, J=29.6 \mathrm{~Hz}) .{ }^{19} \mathrm{~F}$ NMR $\left(376 \mathrm{MHz}, \mathrm{CDCl}_{3}\right) \delta-65.64(\mathrm{t}, J=11.28 \mathrm{~Hz}, 3 \mathrm{~F}) . \mathrm{IR}$ (neat) $\mathrm{cm}^{-1} \tilde{\mathrm{v}}$ : 3361, 2963, 2921, 2851, 2202, 1659, 1633, 1470, 1412, 1261, 1092, 1020, 799, 700, 661. HRMS (EI(+), $70 \mathrm{eV}): \mathrm{C}_{15} \mathrm{H}_{13} \mathrm{OF}_{3} \mathrm{~S}$ [M]: calcd: 298.0639, found: 298.0641. HPLC (Chiralcel OD-H column, hexanes: $i-\mathrm{PrOH}=98: 2,0.8 \mathrm{~mL} / \mathrm{min}, 210$ $\mathrm{nm}), \mathrm{t}_{\text {minor }}=16.6 \mathrm{~min}, \mathrm{t}_{\text {major }}=17.5 \mathrm{~min}, \mathrm{ee}=86 \% .[\alpha]_{\mathrm{D}}{ }^{30}=3.7,\left(\mathrm{c}=0.2, \mathrm{CH}_{2} \mathrm{Cl}_{2}\right)$.

\section{References}

[1] Xiong, Y.; Zhang, G. Enantioselective Synthesis of Quaternary Stereocenters via Chromium Catalysis. Org. Lett. 2016, 18, 5094.

[2] Isolated yield.

\section{Spectrogram information}

\section{(1). Copies of NMR specta and HPLC chromatographs}

(L) $-{ }^{1} \mathrm{H}$ NMR 


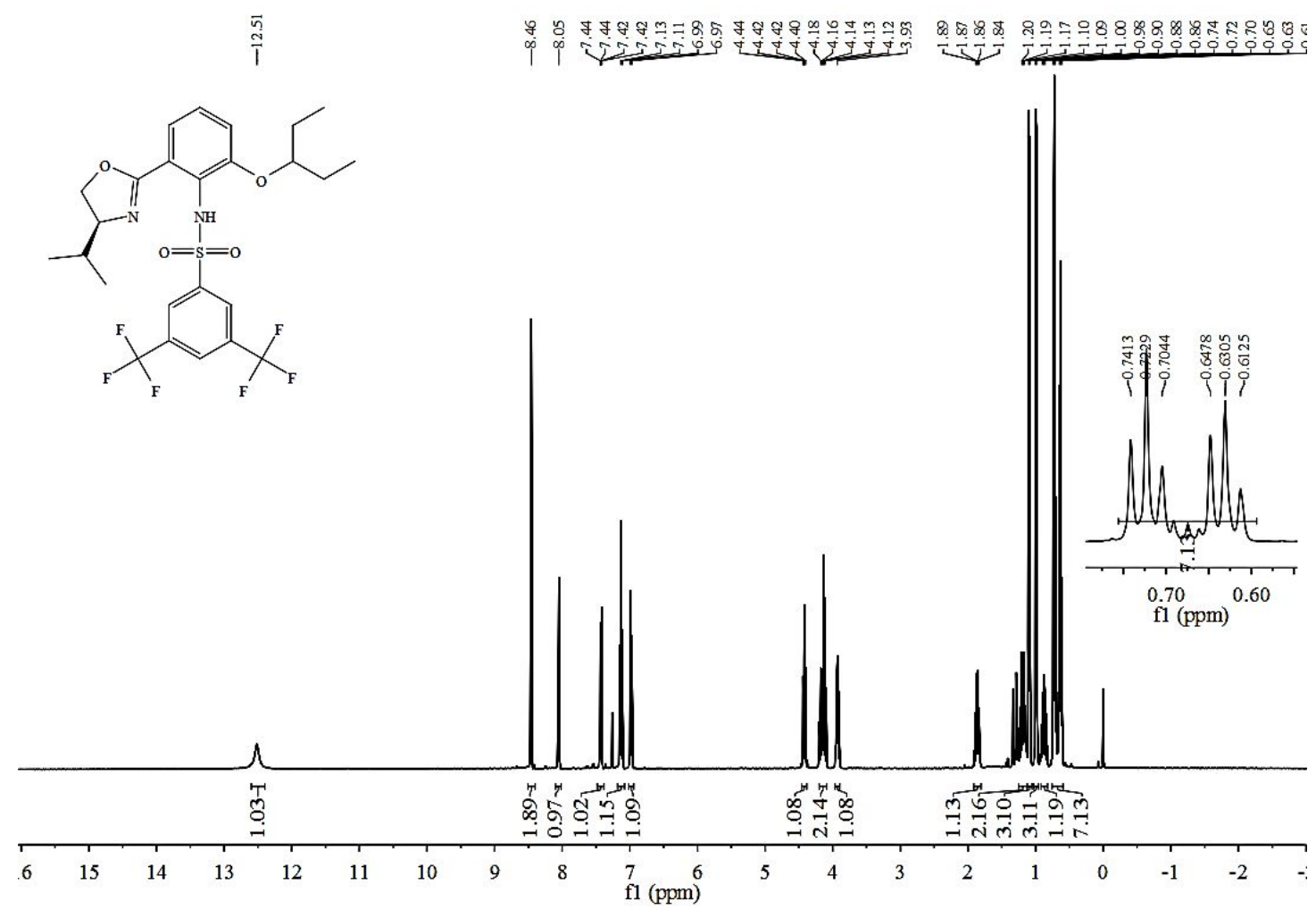

(L)- ${ }^{13} \mathrm{C}$ NMR

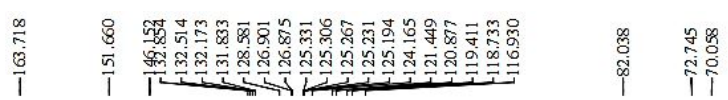

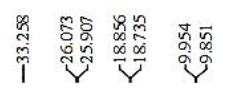<smiles>CCC(CC)Oc1cccc(C2=N[C@@H](C(C)C)CO2)c1NS(=O)(=O)c1cc(C(F)(F)F)cc(C(F)(F)F)c1</smiles>
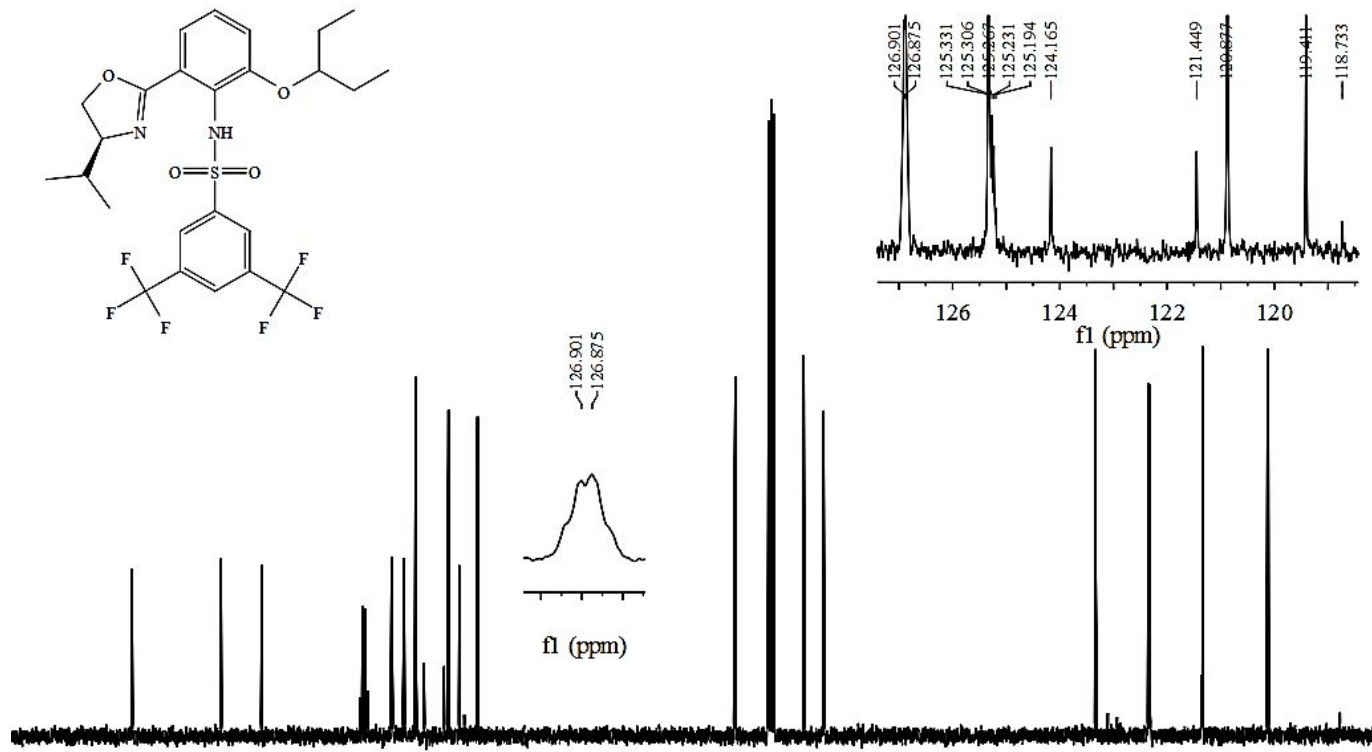

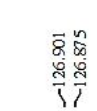

$\begin{array}{llllllllll}30 & 170 & 160 & 150 & 140 & 130 & 120 & 110 & 100 & 90 \begin{array}{l}80 \\ \text { f1 (ppm })\end{array}\end{array}$

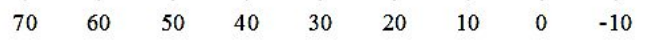

(L) $-{ }^{19}$ F NMR 


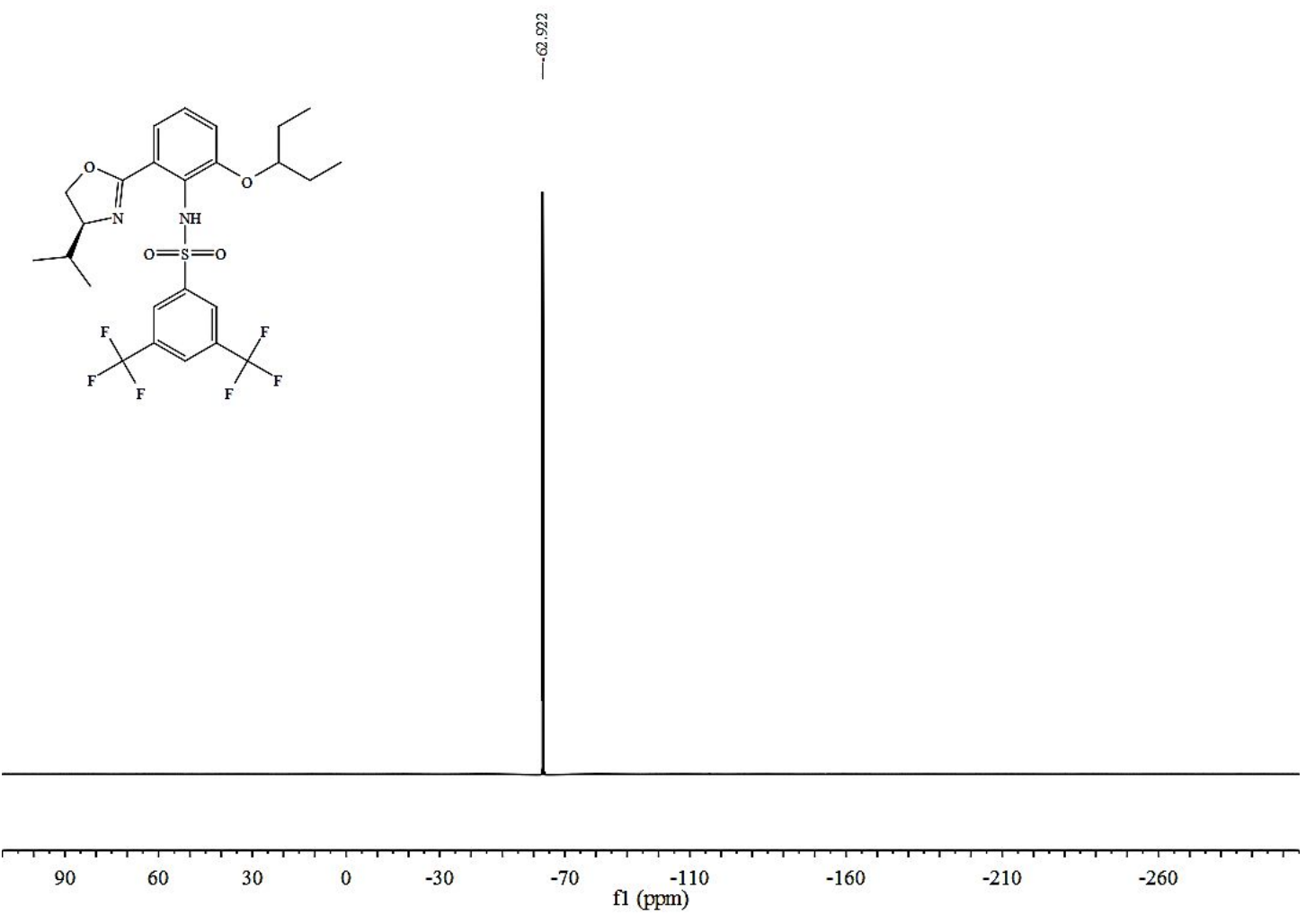

(S,E)-6,6-dimethyl-1,4-diphenylhept-4-en-3-ol (1d) - ${ }^{1} \mathrm{H}$ NMR
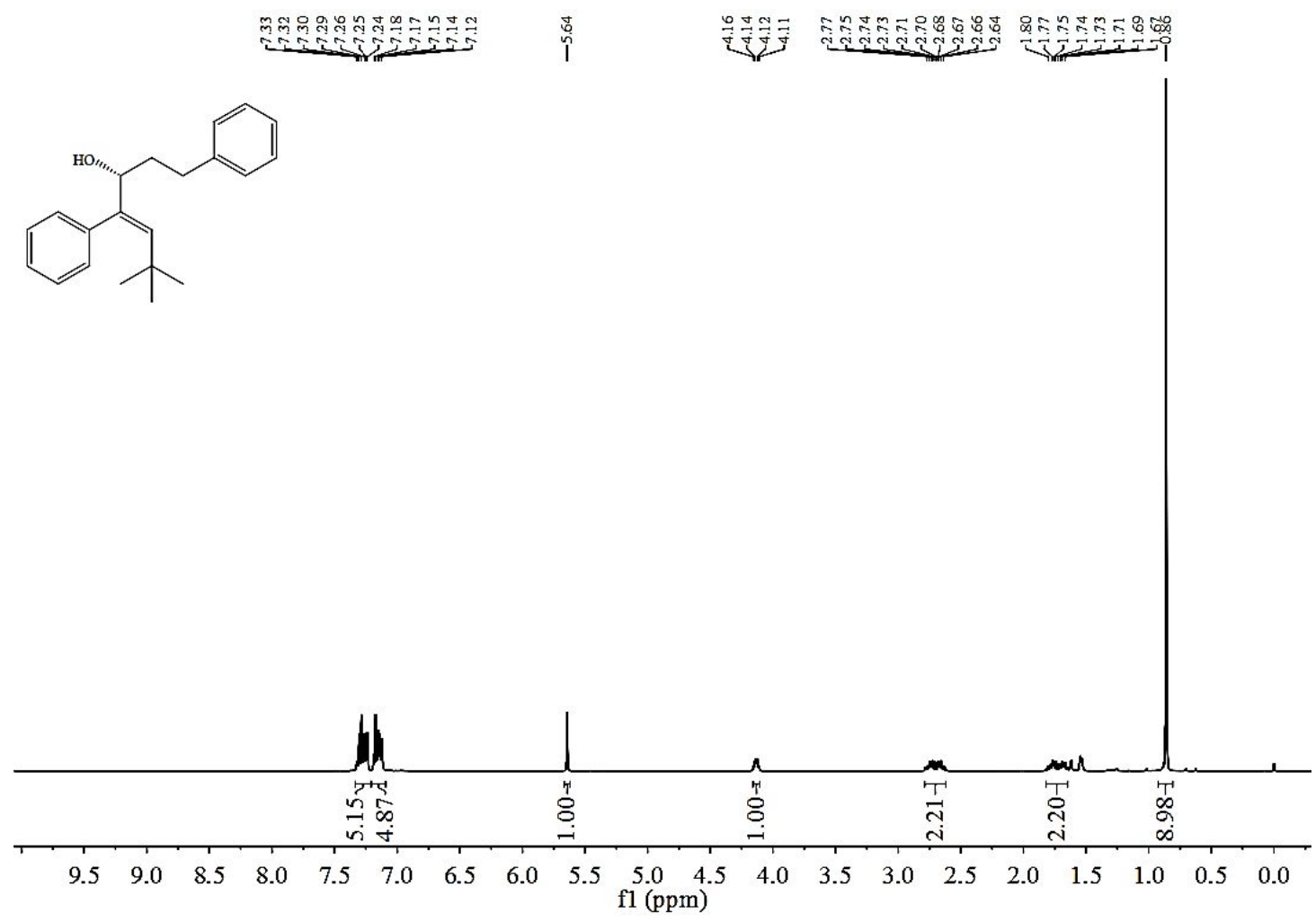

(S,E)-6,6-dimethyl-1,4-diphenylhept-4-en-3-ol (1d) - ${ }^{13}$ C NMR 


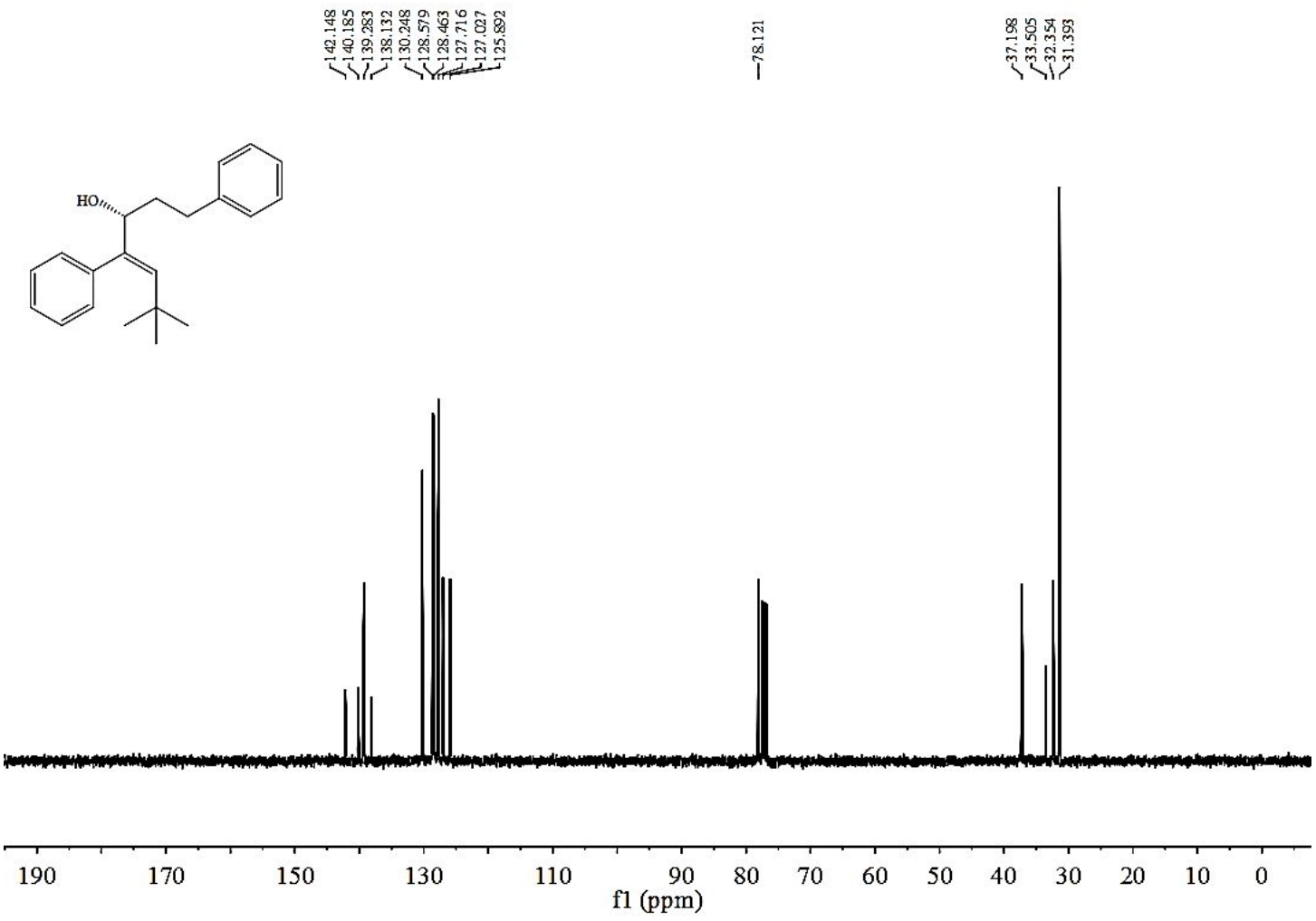

1d-HPLC (racemic)

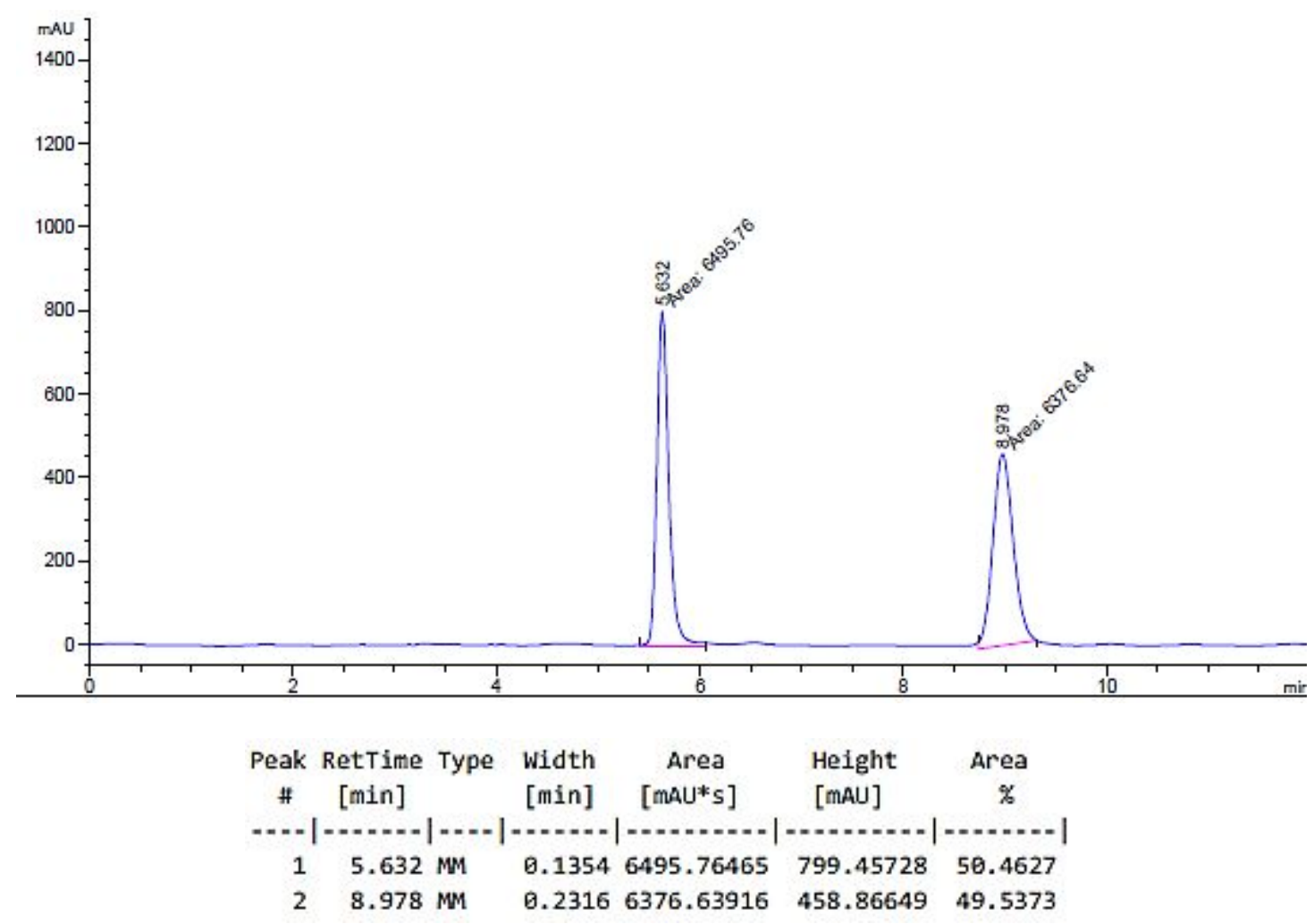

1d-HPLC (95\%) 


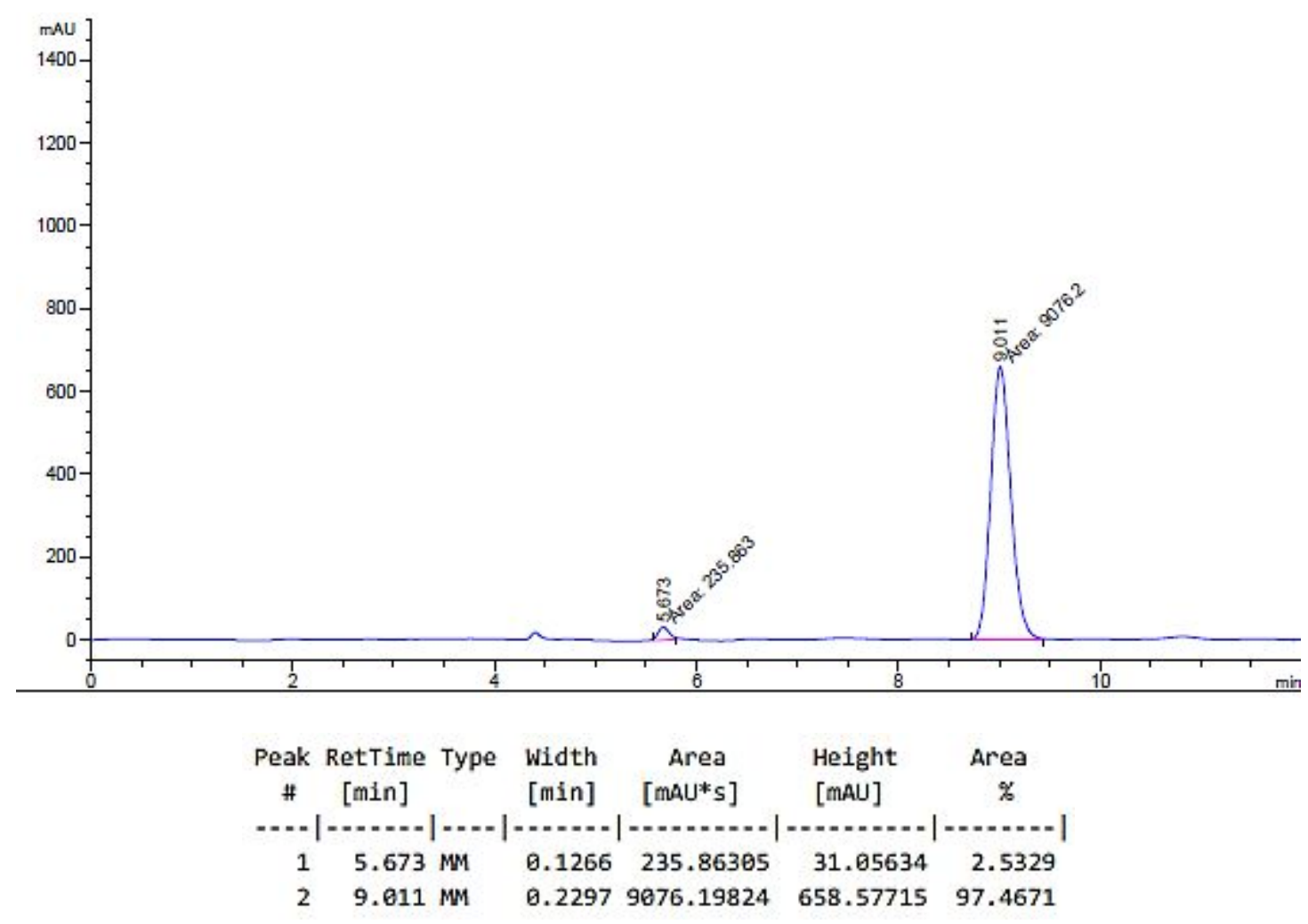

(S,E)-2,2-dimethyl-4-phenyldec-3-en-5-ol (2d) - '1 H NMR

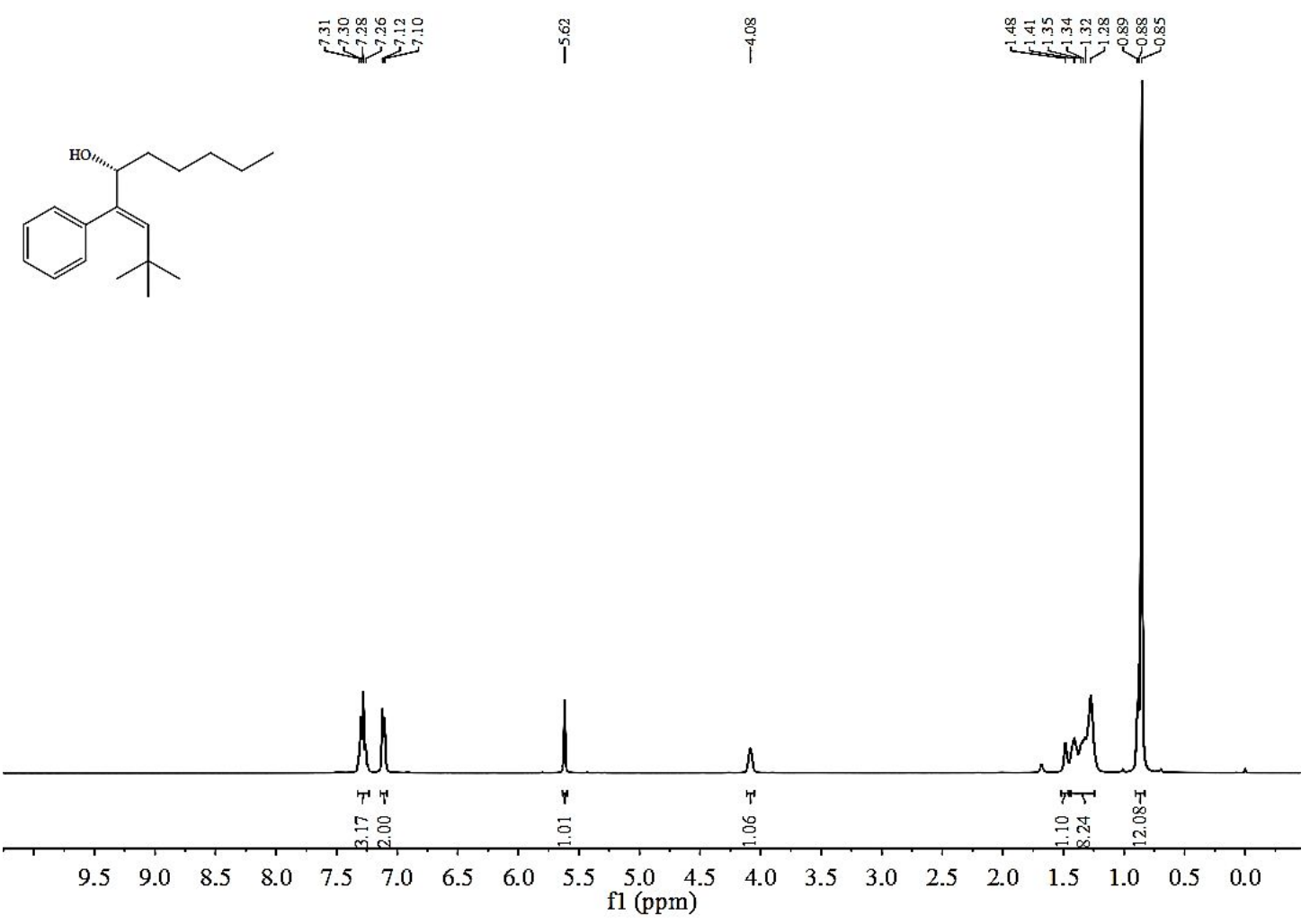

(S,E)-2,2-dimethyl-4-phenyldec-3-en-5-ol (2d) ${ }^{13}$ C NMR 

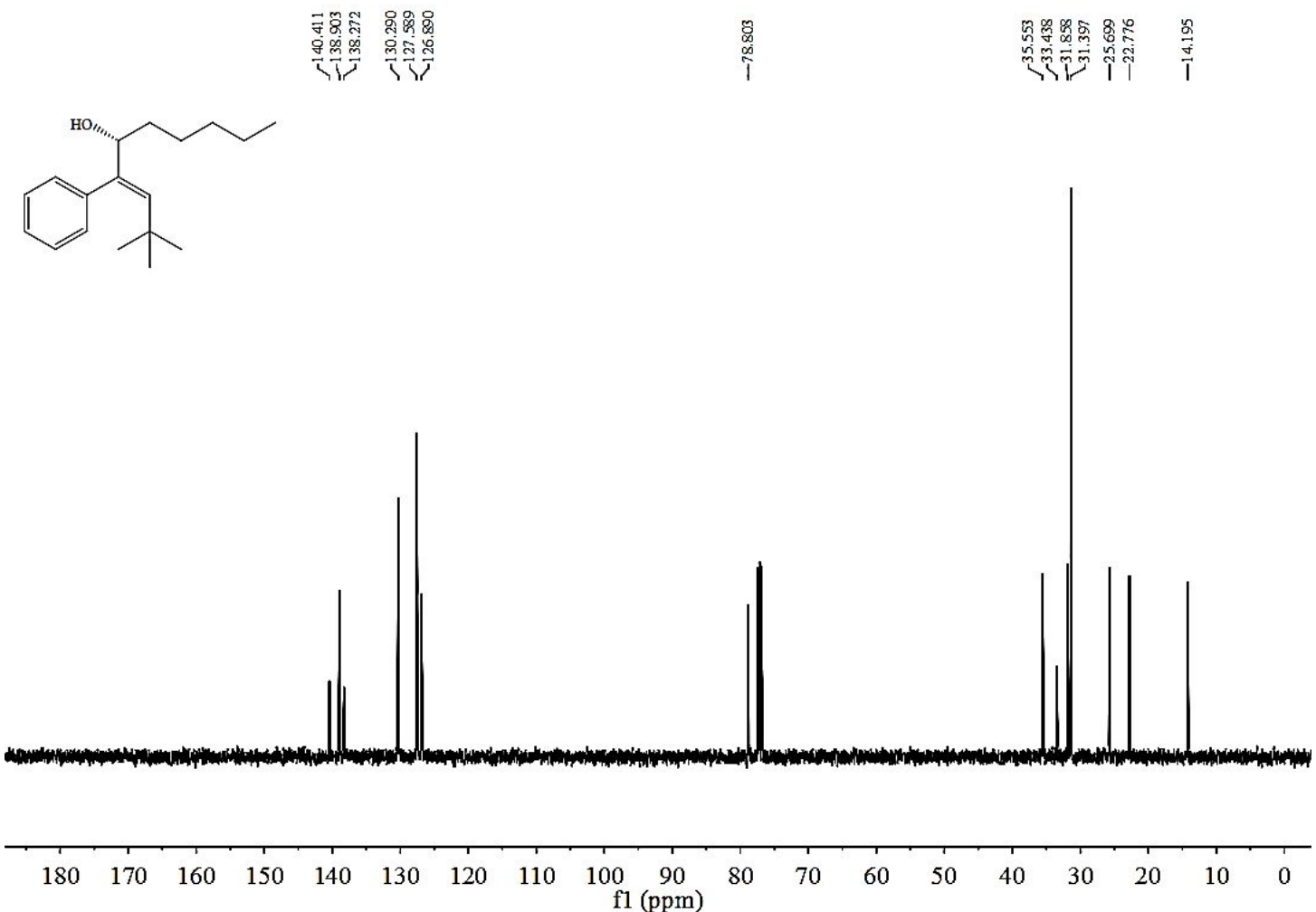

\section{2d-HPLC (racemic)}

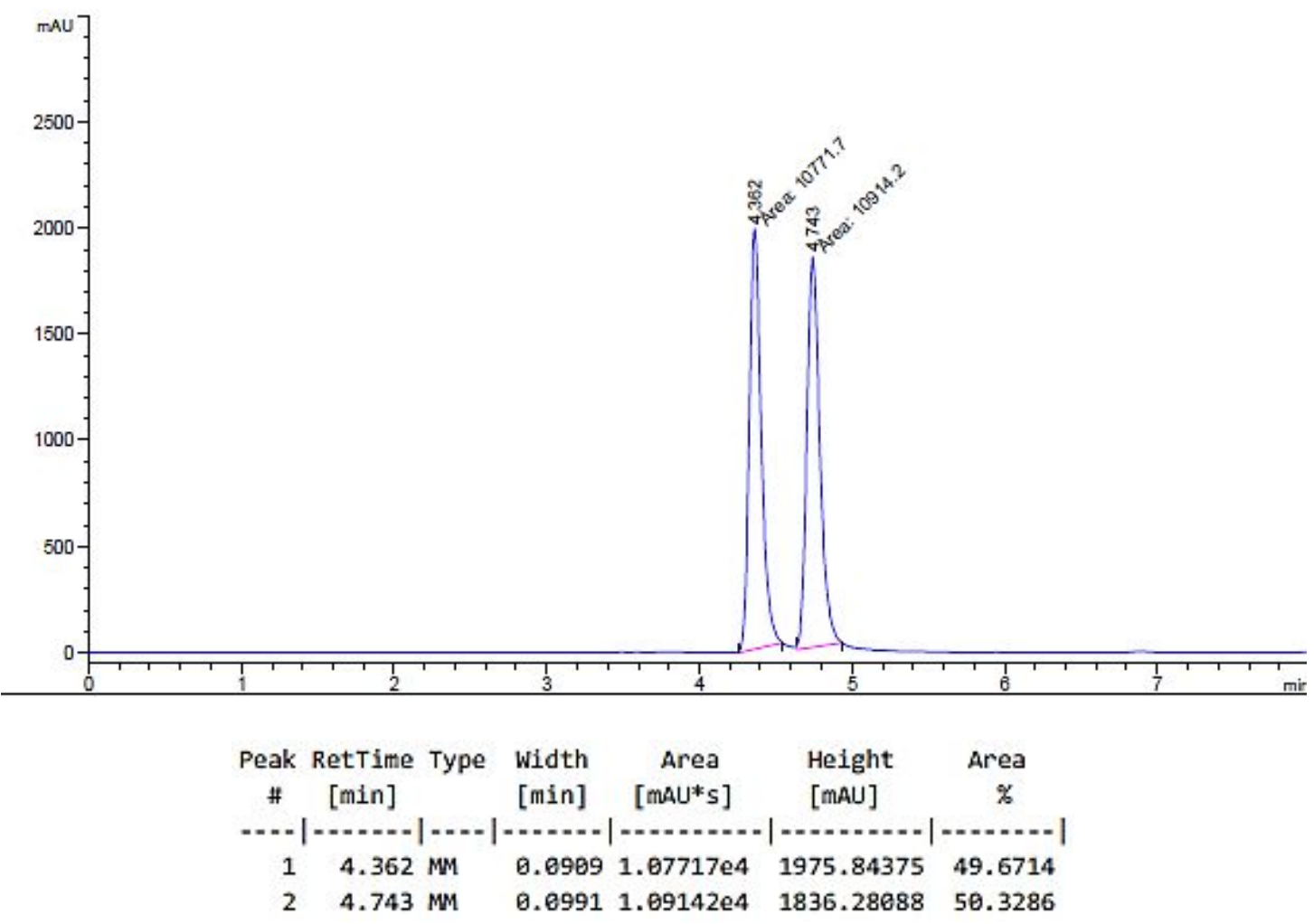

2d-HPLC (94\%) 


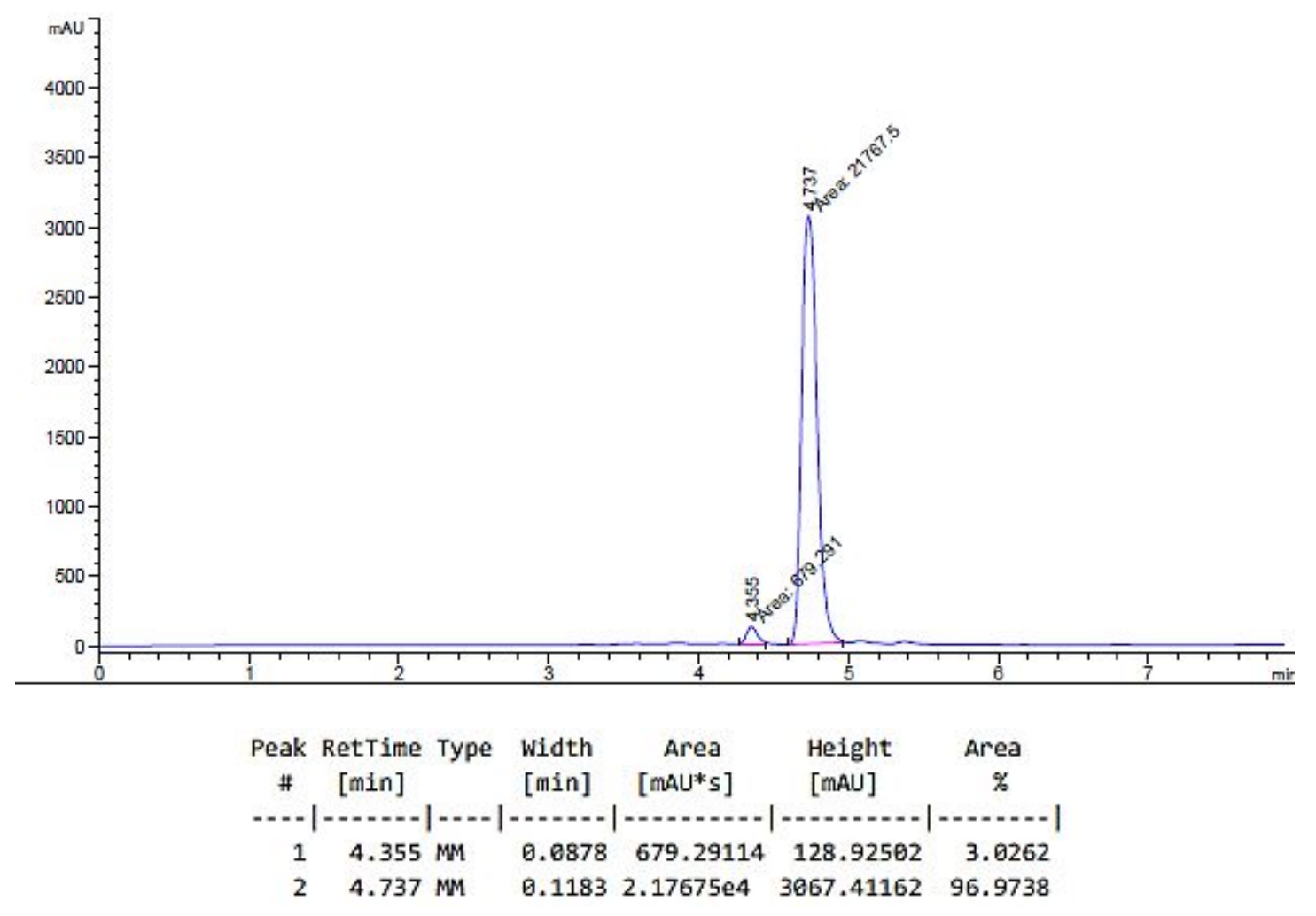

(S,E)-1-(2,2-dimethyl-1,1-diphenylpropoxy)-7,7-dimethyl-5-phenyloct-5-en-4-ol (3d) $-{ }^{1} \mathrm{H}$ NMR

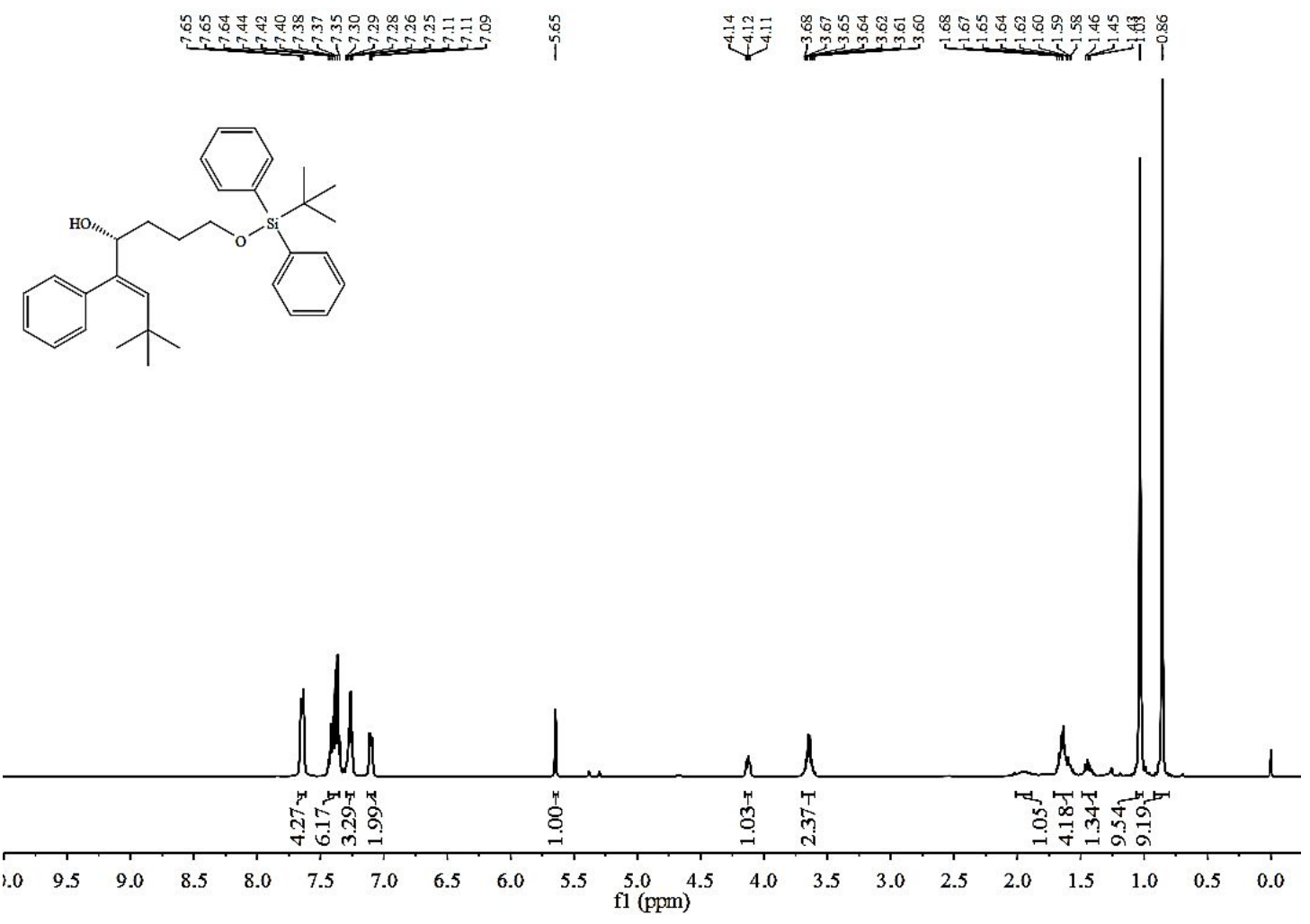

(S,E)-1-(2,2-dimethyl-1,1-diphenylpropoxy)-7,7-dimethyl-5-phenyloct-5-en-4-ol (3d) 
${ }^{-13} \mathrm{C}$ NMR
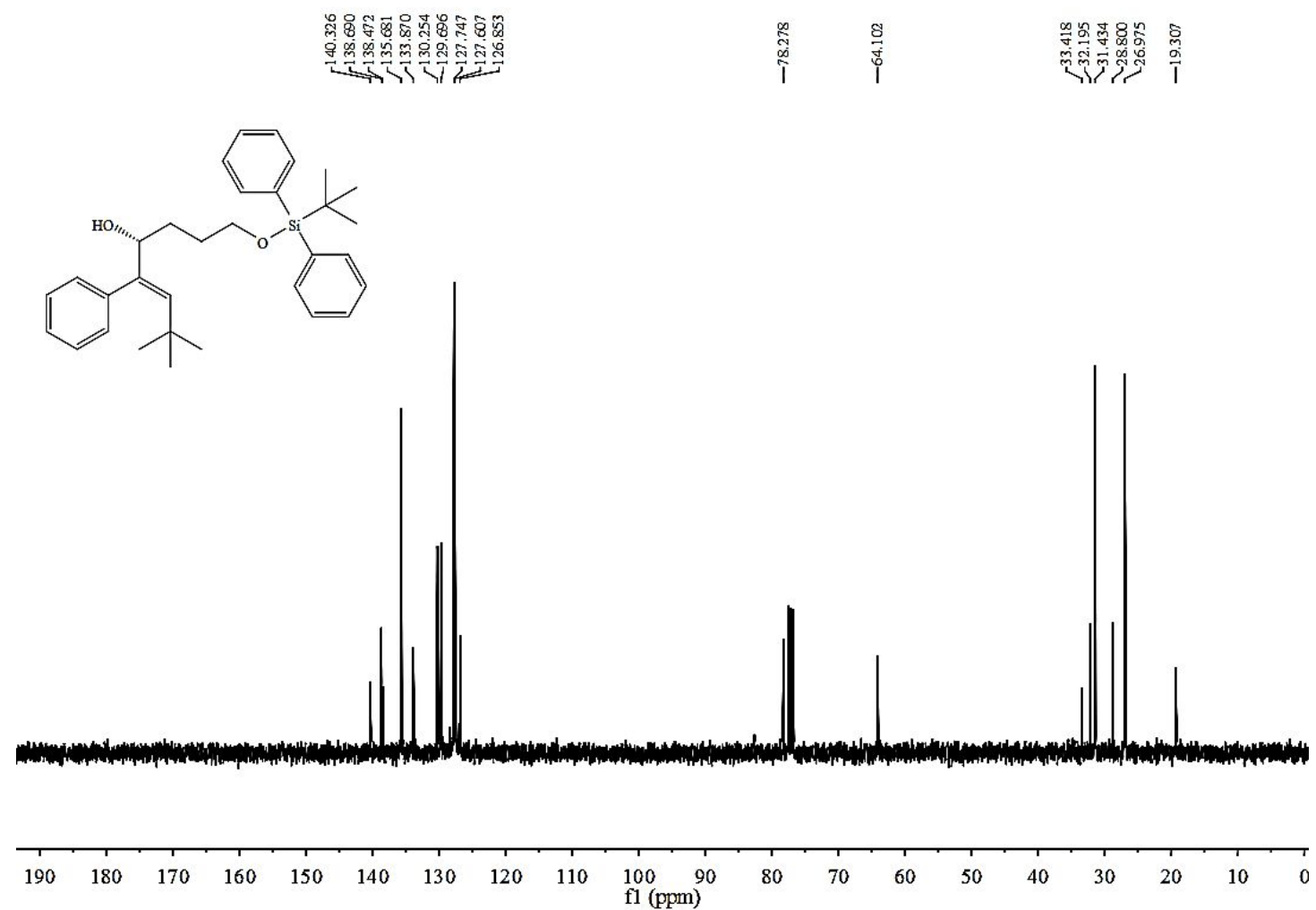

3d-HPLC (racemic)

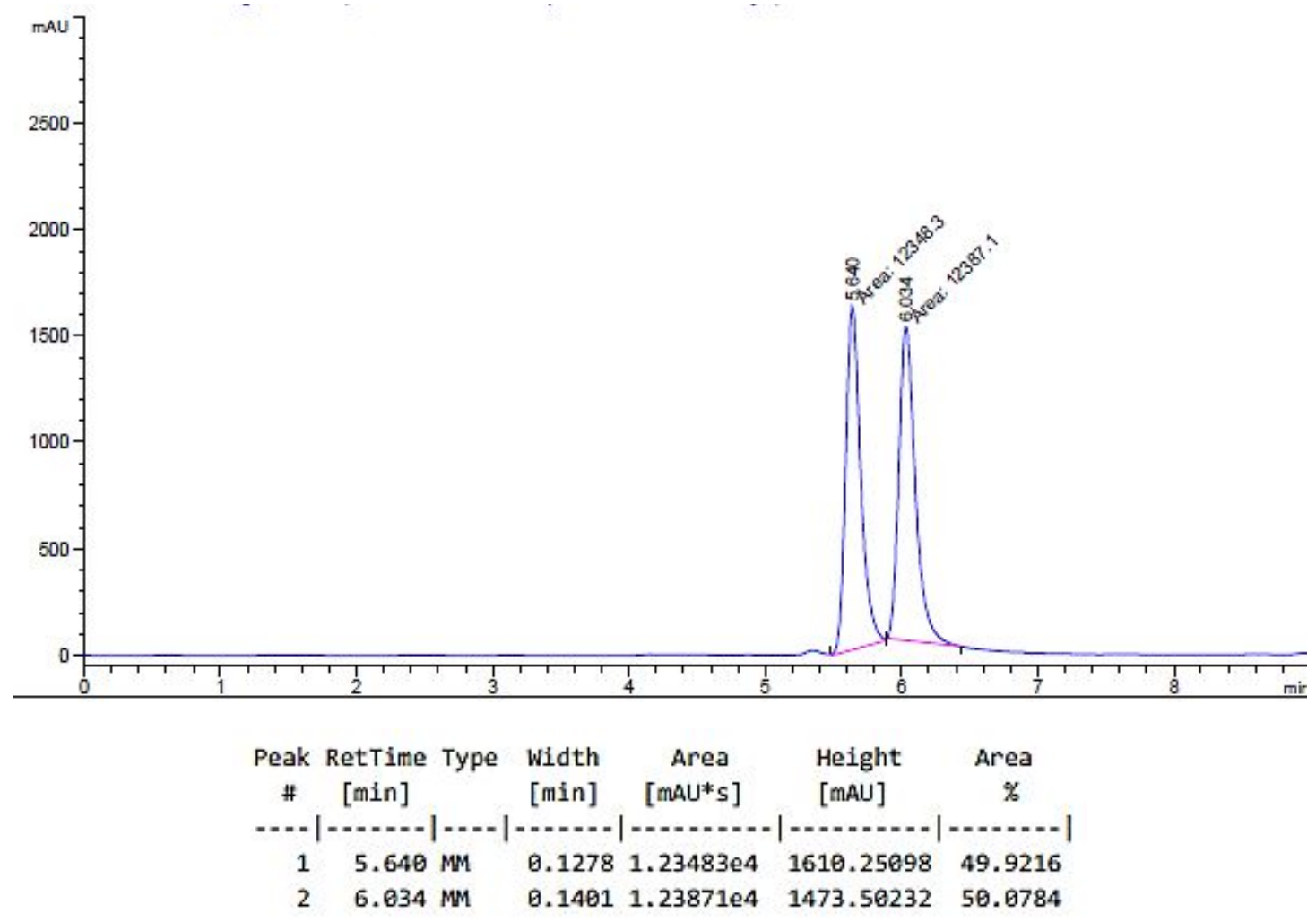

3d-HPLC (racemic) 


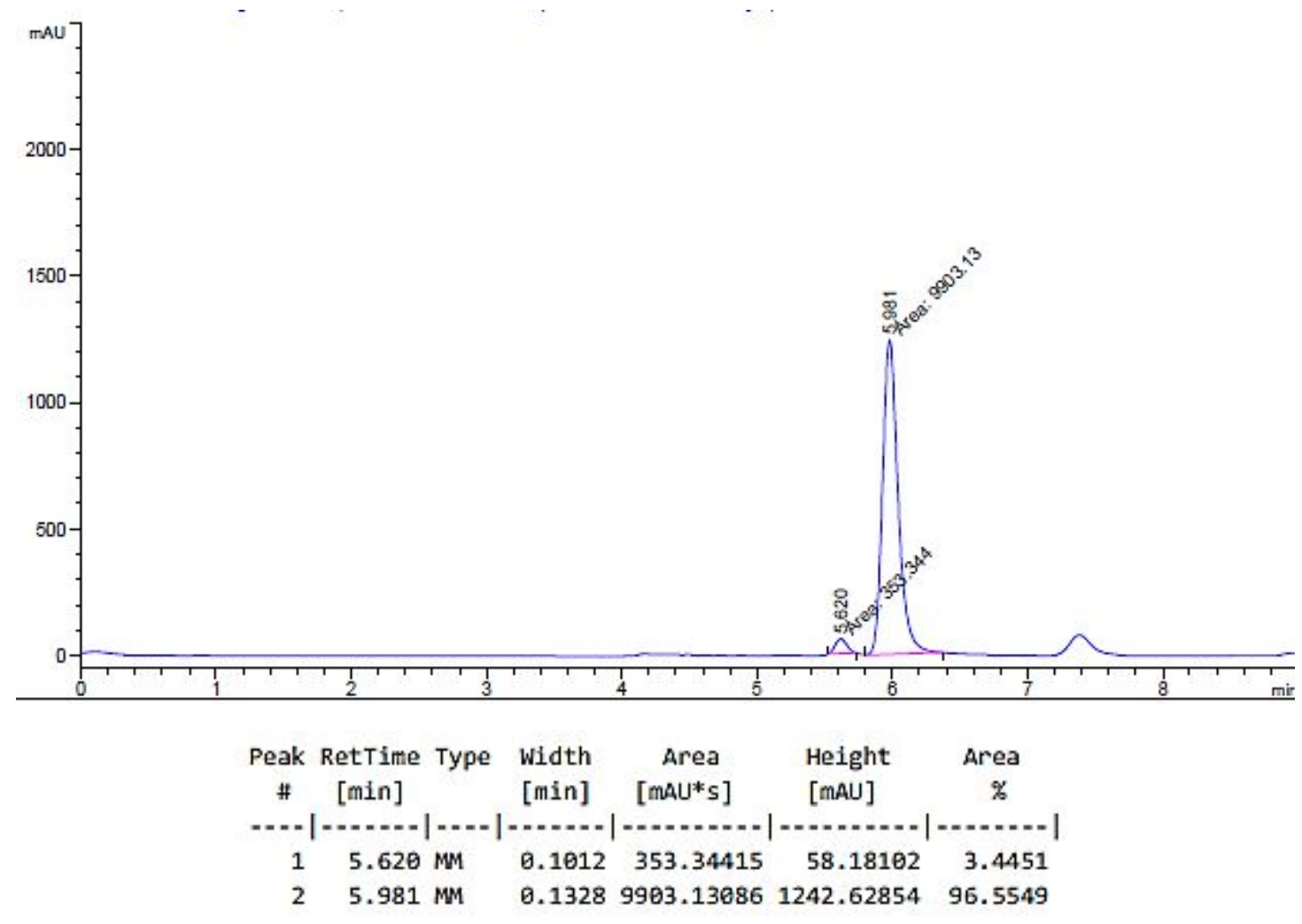

(S,E)-6-methyl-1,4-diphenylhept-4-en-3-ol (4d) - ${ }^{1} \mathrm{H}$ NMR
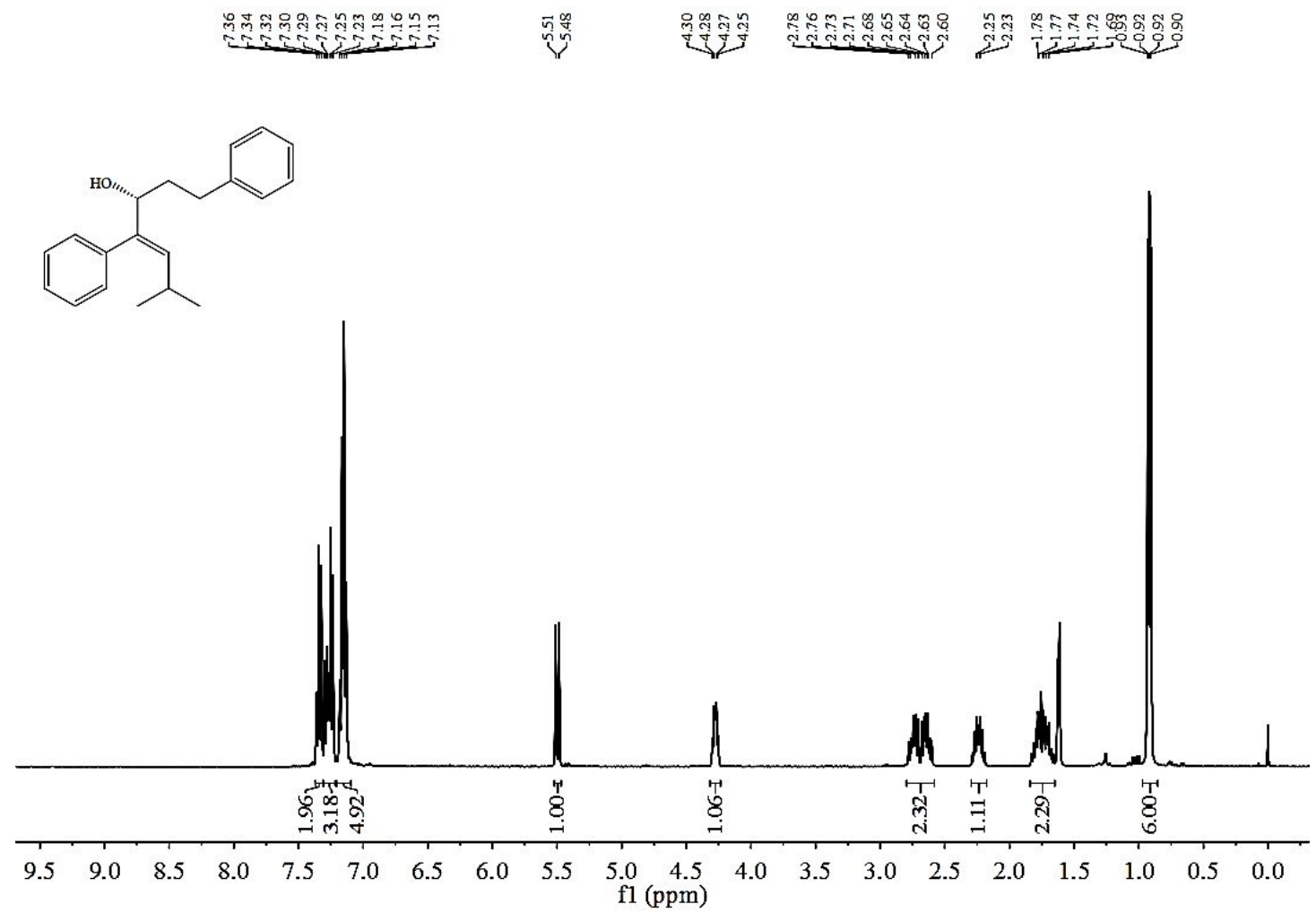

(S,E)-6-methyl-1,4-diphenylhept-4-en-3-ol (4d) - ${ }^{13}$ C NMR 


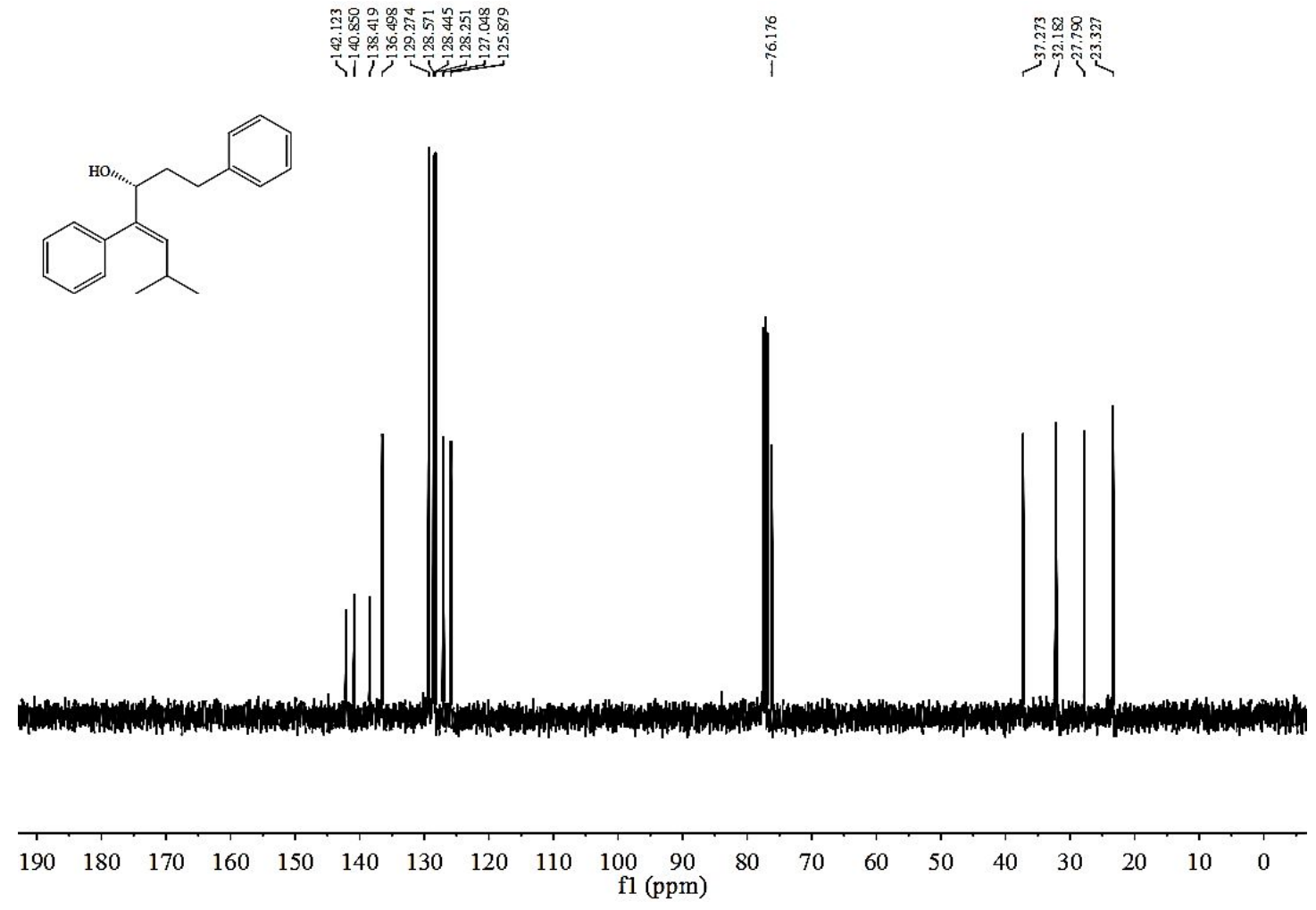

\section{4d-HPLC (racemic)}

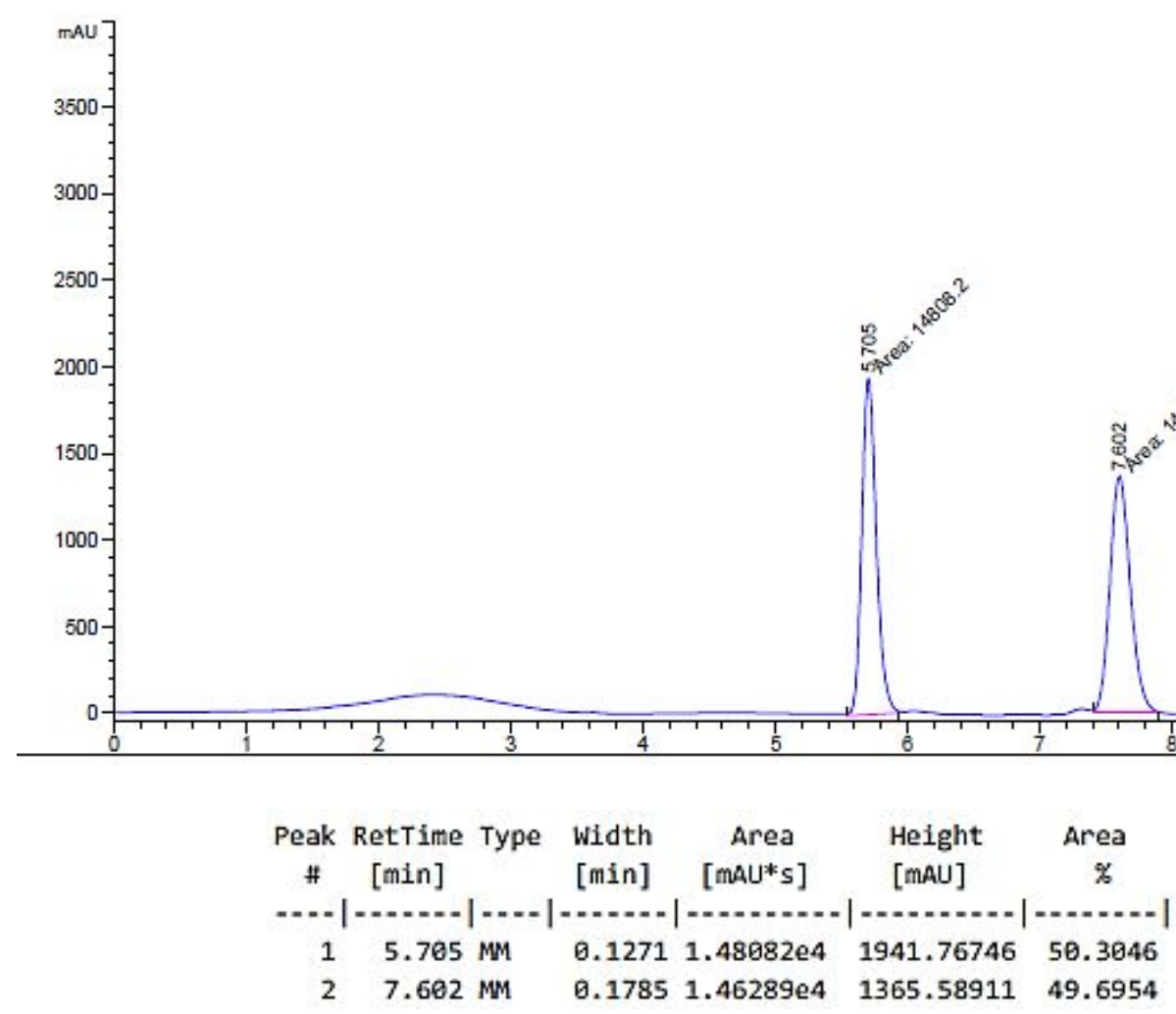

4d-HPLC (95\%) 


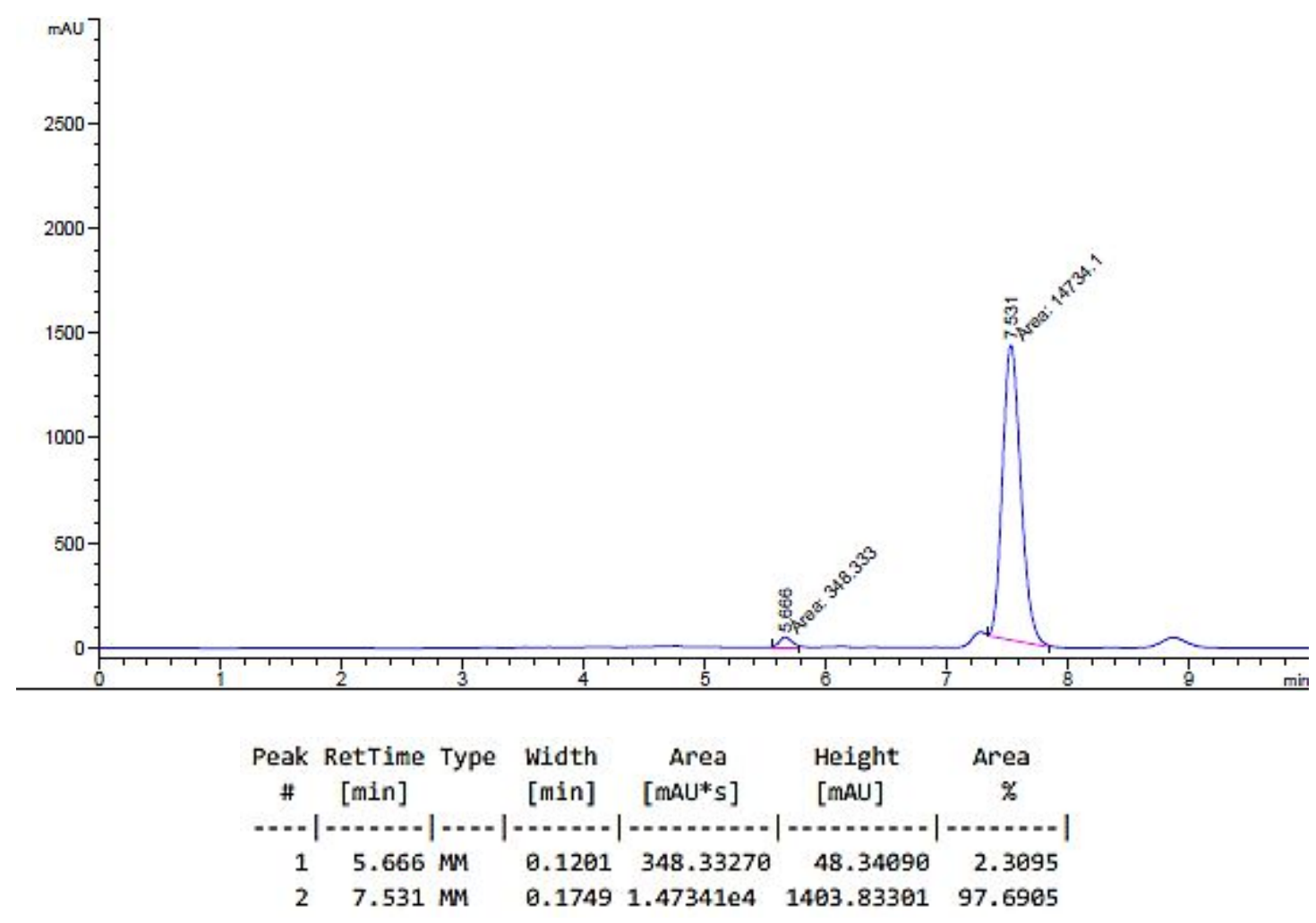

(S,E)-2-methyl-4-phenyldec-3-en-5-ol (5d) - ${ }^{1} \mathrm{H}$ NMR

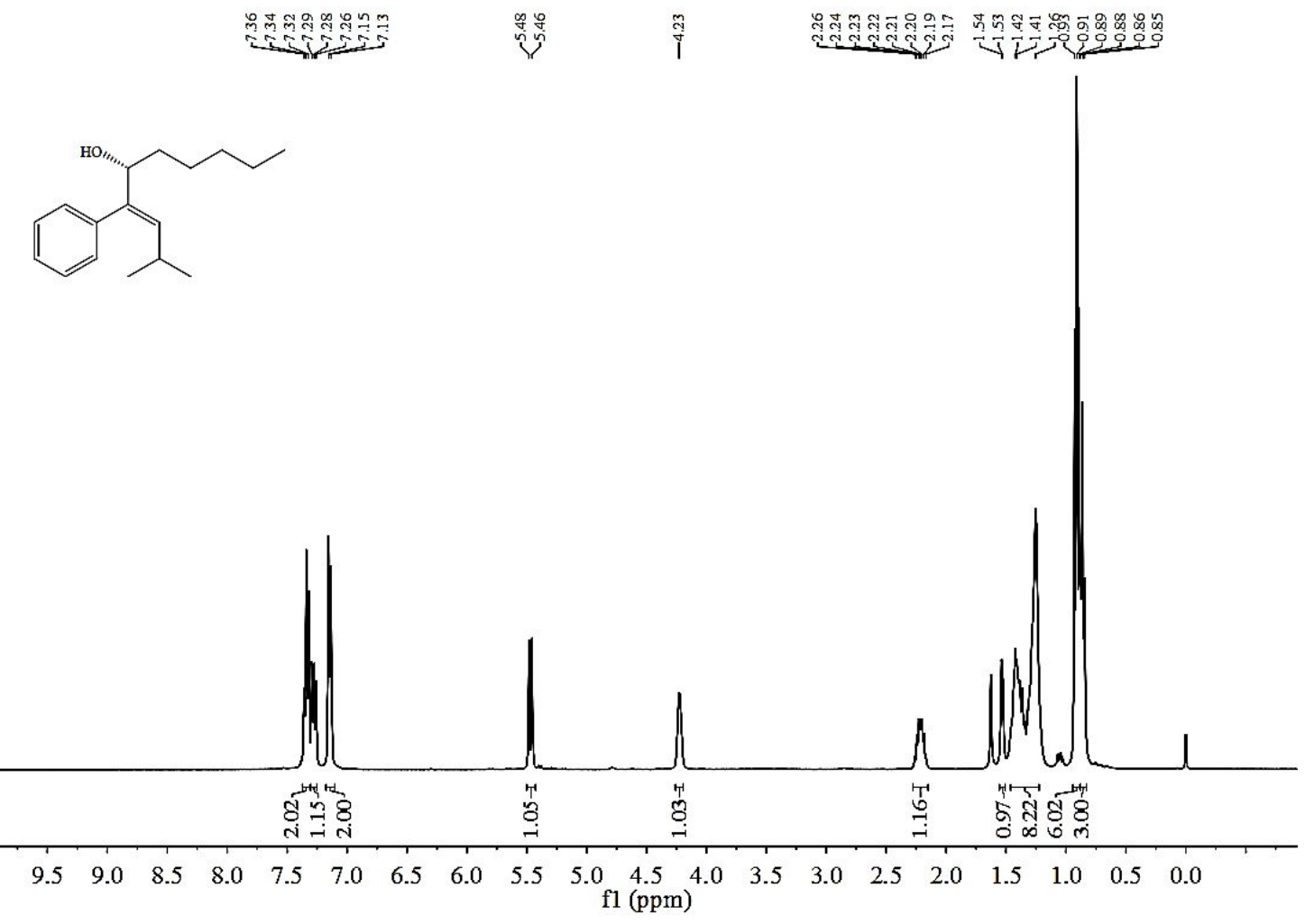

(S,E)-2-methyl-4-phenyldec-3-en-5-ol (5d) - ${ }^{13}$ C NMR 


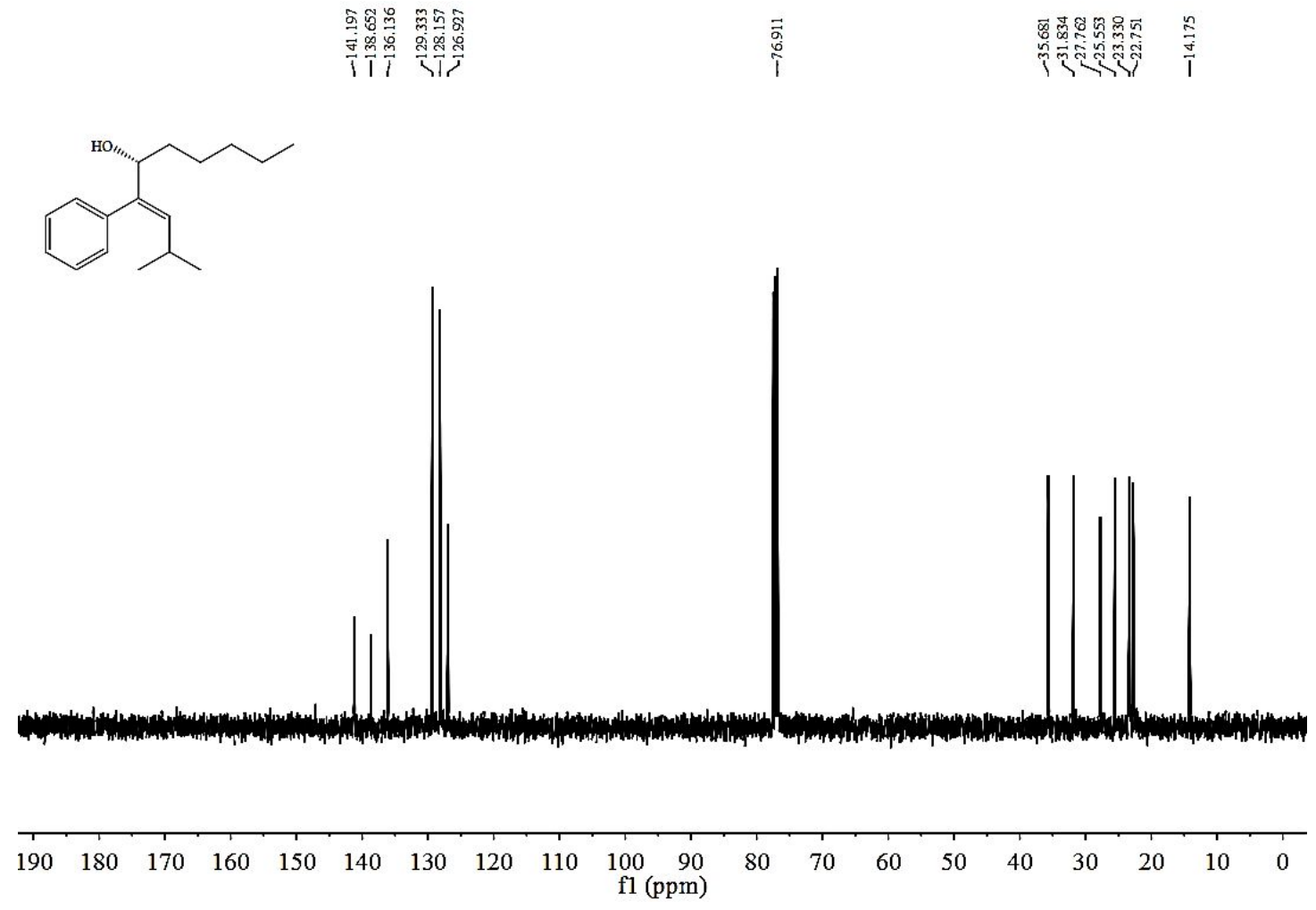

\section{5d-HPLC (racemic)}

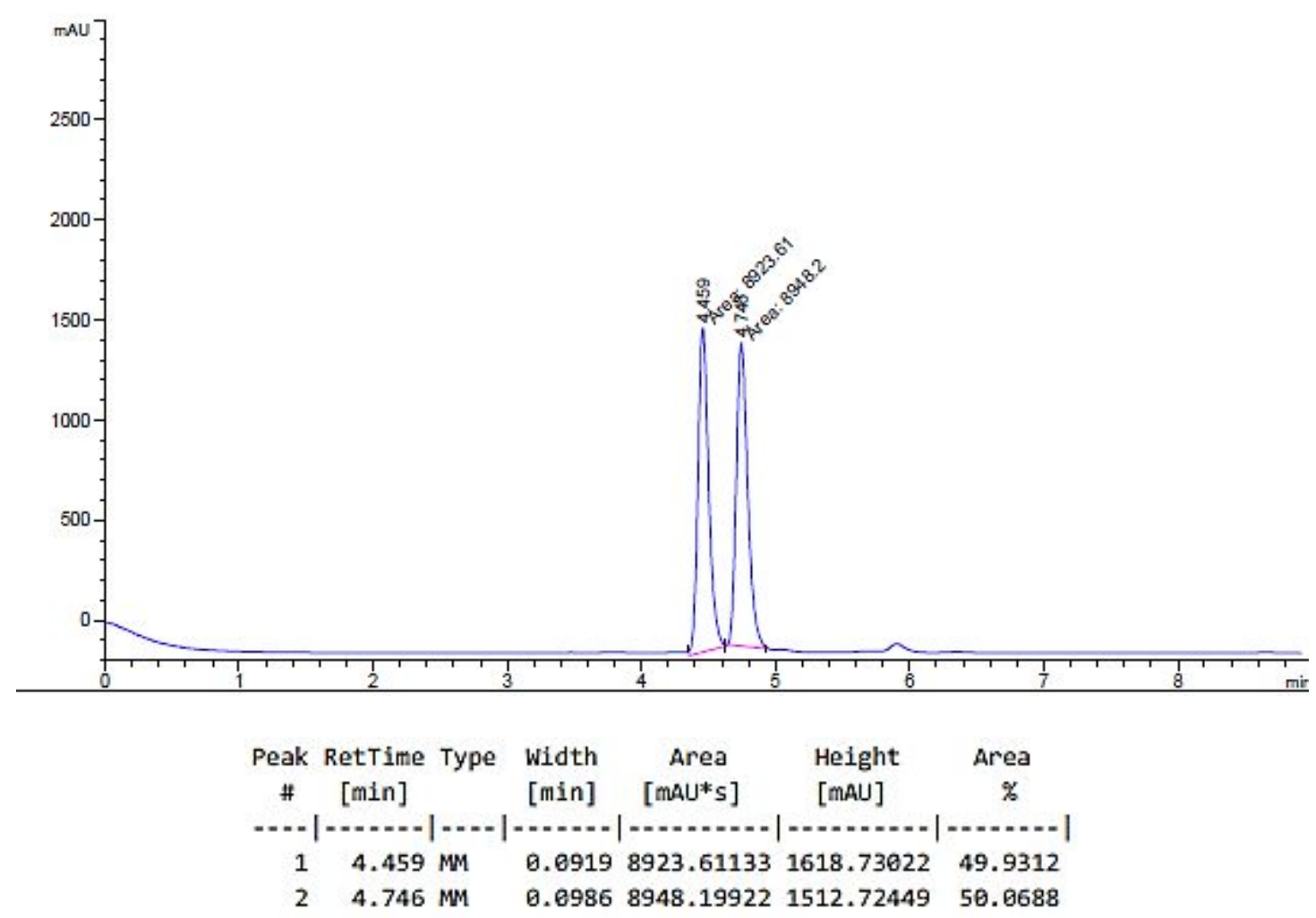

5d-HPLC (95\%) 


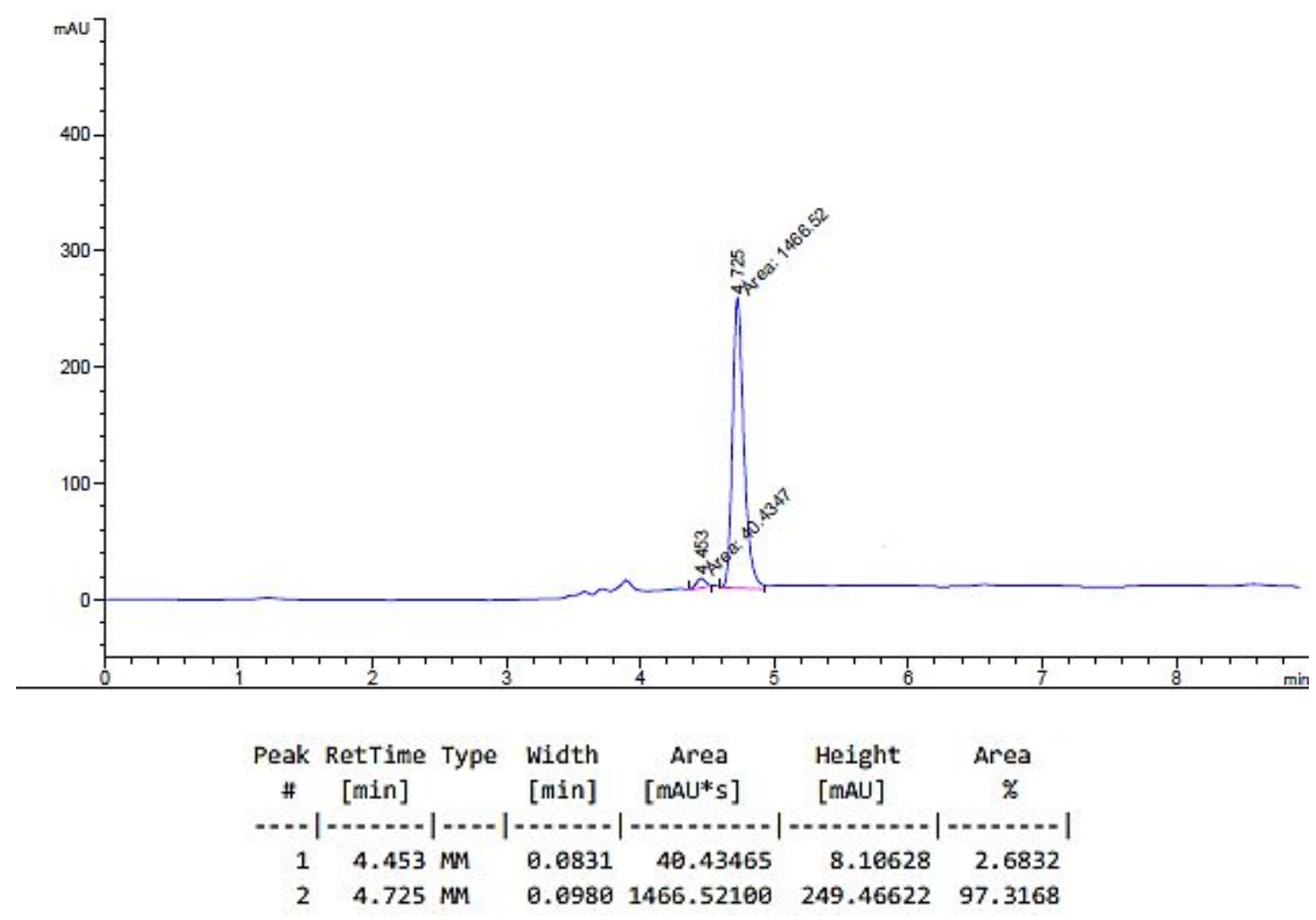

(S,E)-3-cyclohexyl-1,2-diphenylprop-2-en-1-ol (6d) - ${ }^{-1} \mathrm{H}$ NMR

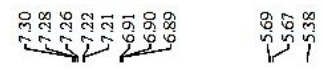

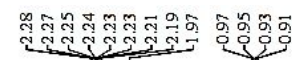
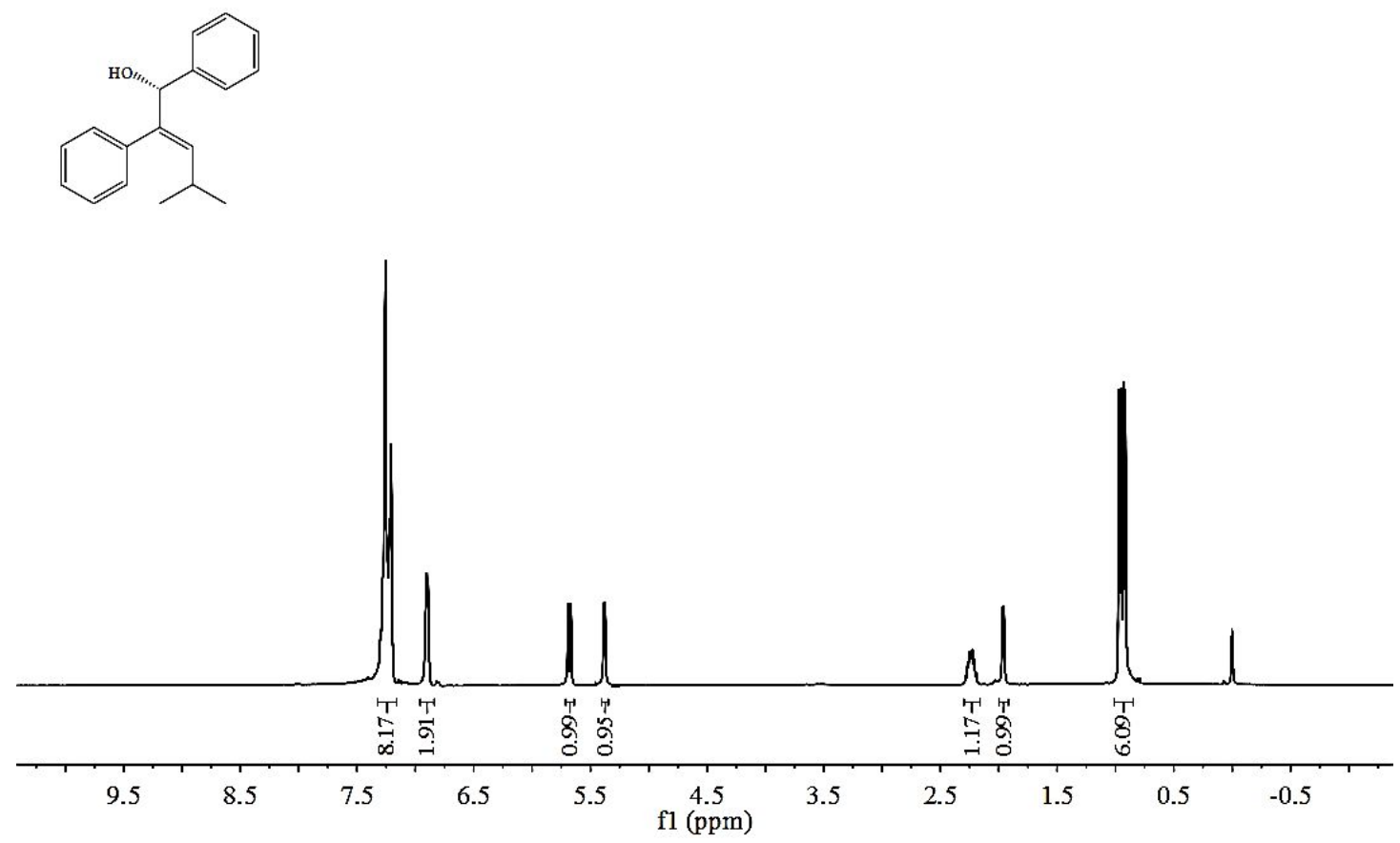

(S,E)-3-cyclohexyl-1,2-diphenylprop-2-en-1-ol (6d) - ${ }^{13} \mathrm{C}$ NMR 


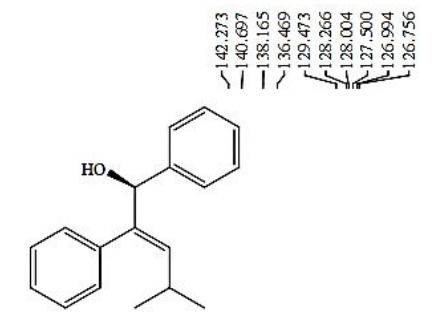

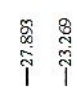

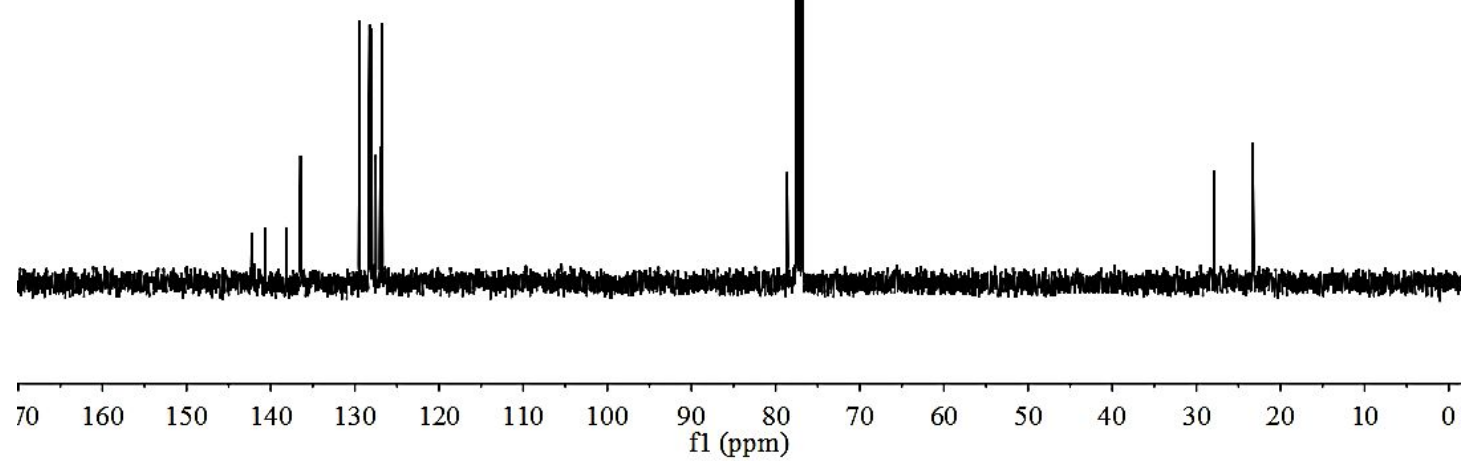

6d-HPLC (racemic)

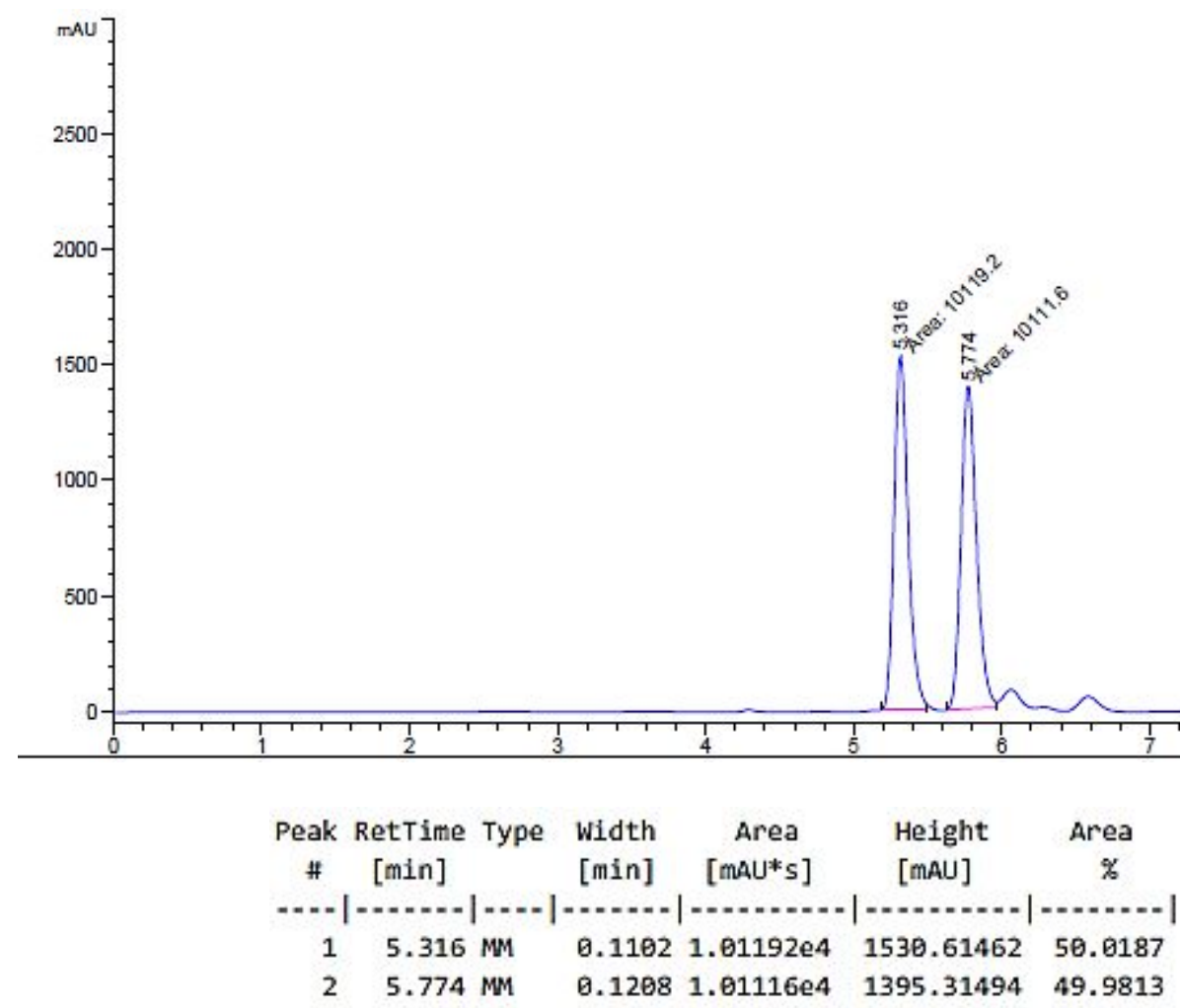

6d-HPLC (96\%) 


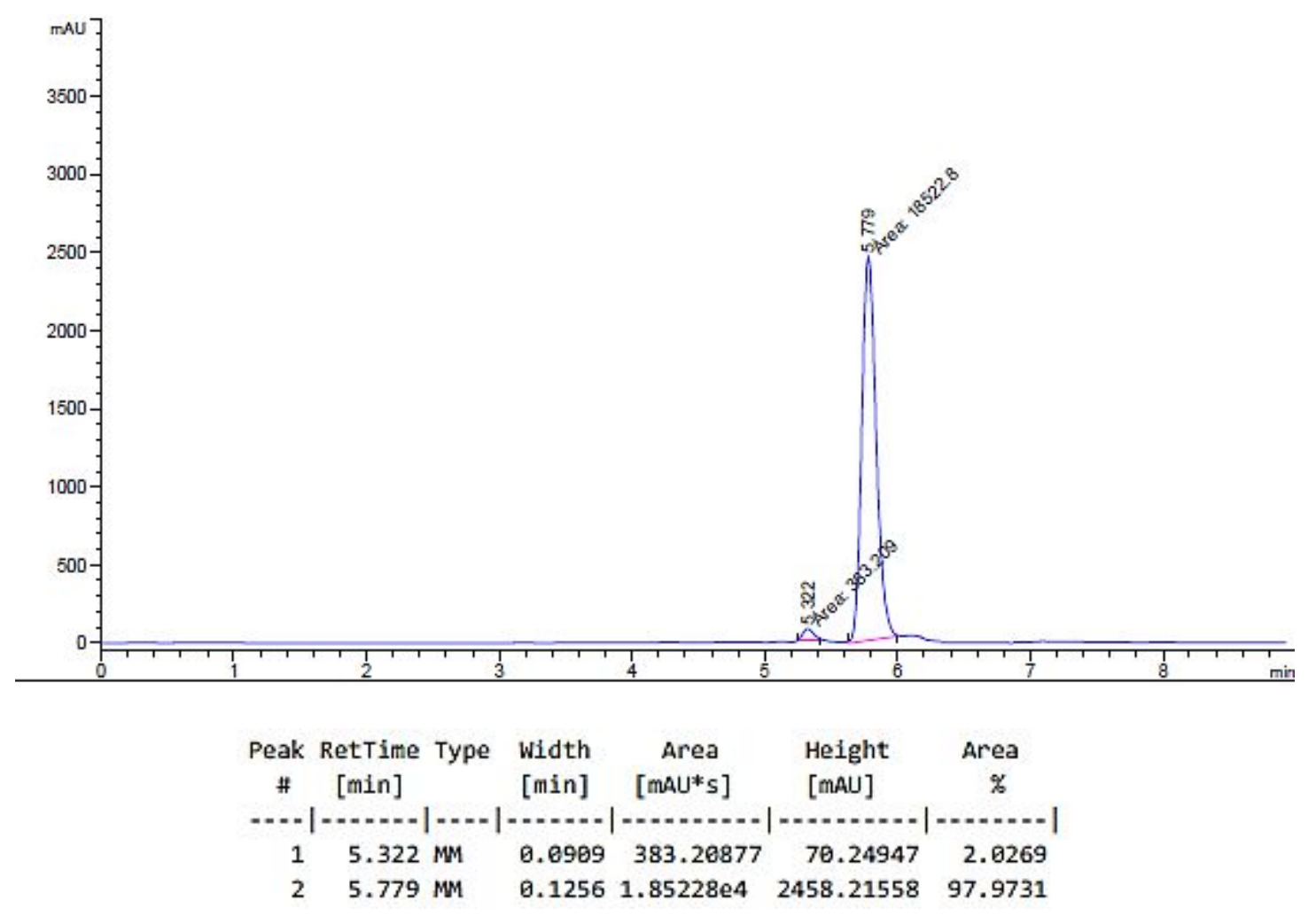

(S,E)-4,4-dimethyl-1,2-diphenylpent-2-en-1-ol (7d) )- ${ }^{1} \mathrm{H}$ NMR
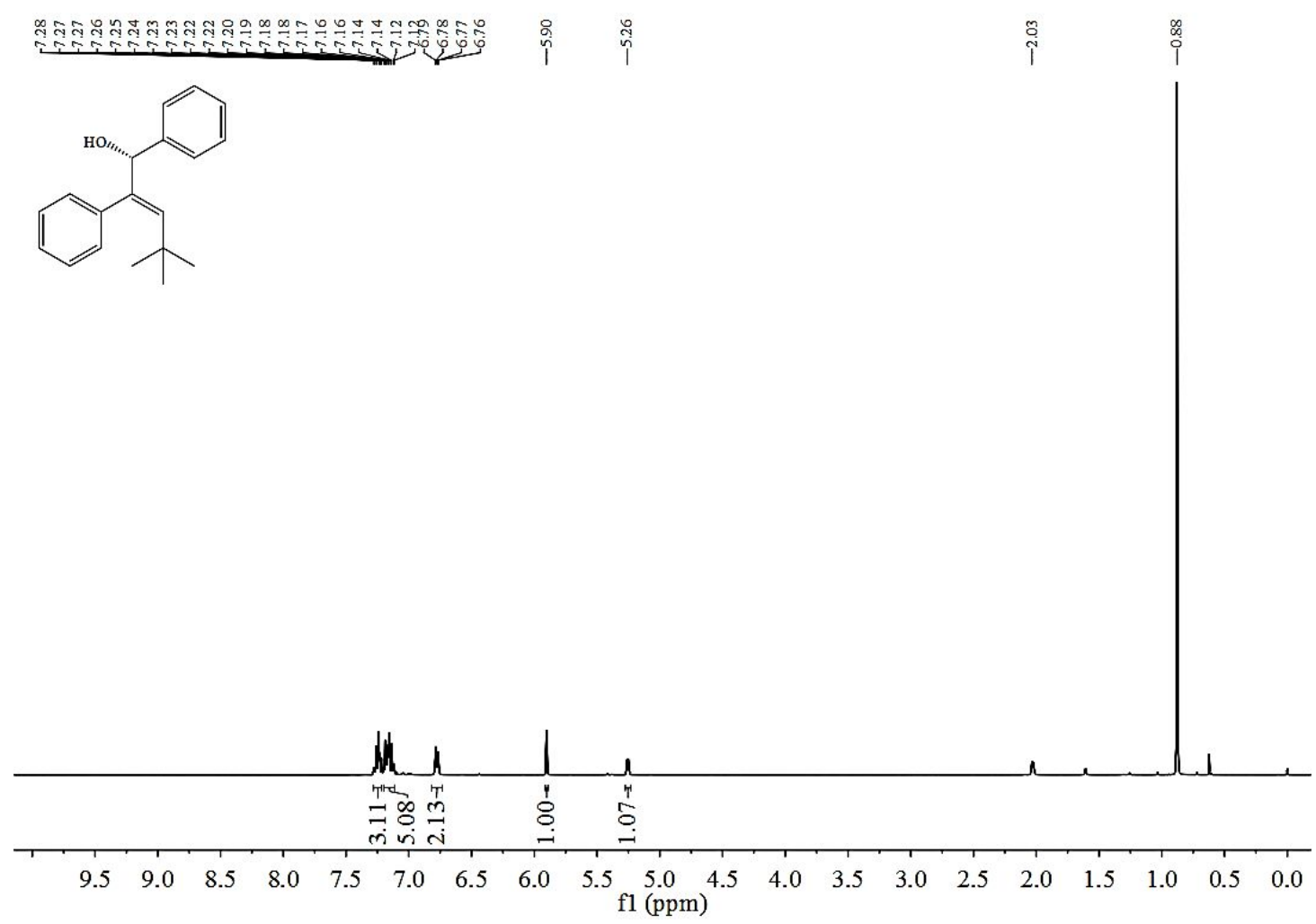

(S,E)-4,4-dimethyl-1,2-diphenylpent-2-en-1-ol (7d) )- ${ }^{13} \mathrm{C}$ NMR 

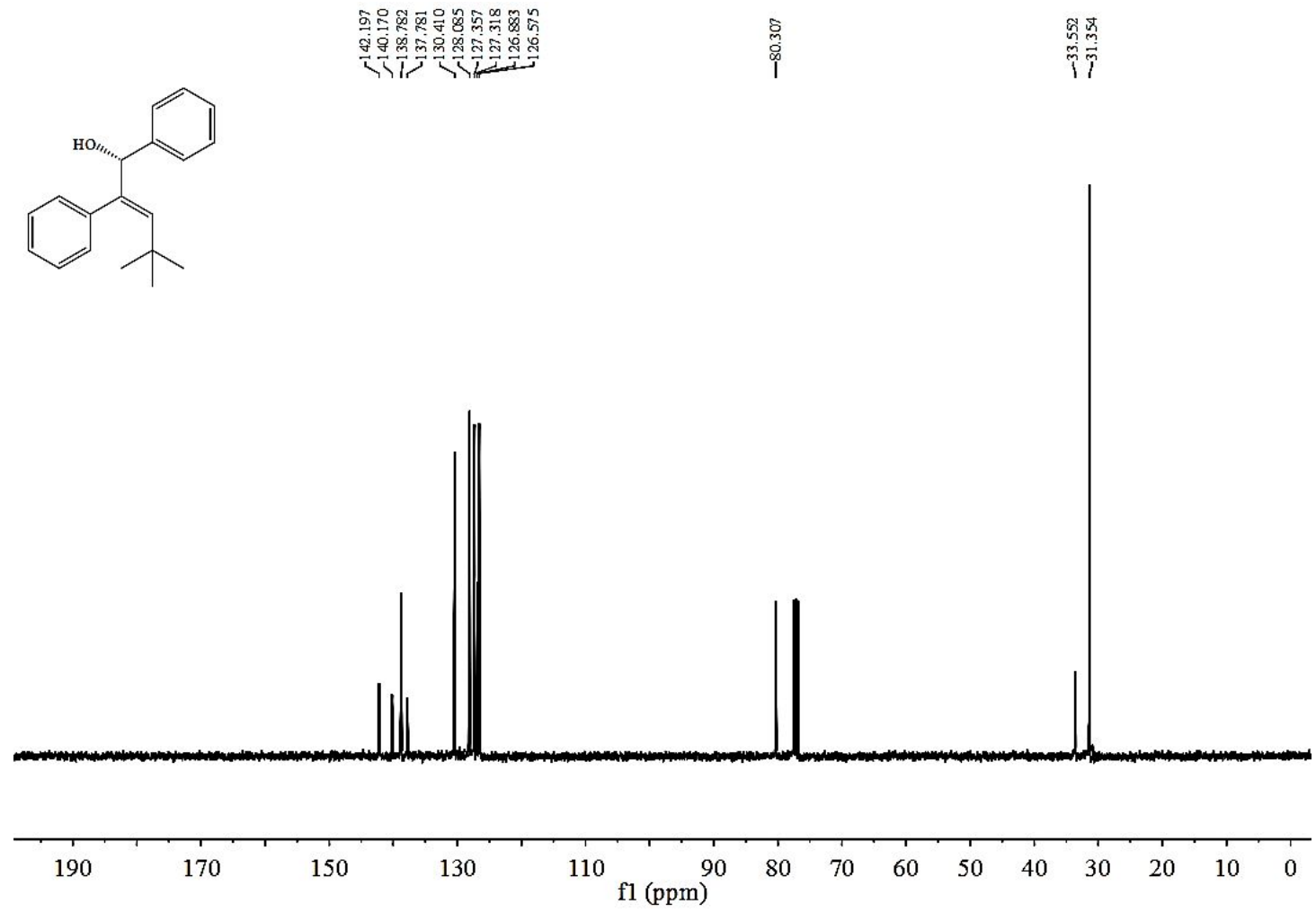

7d-HPLC (racemic)

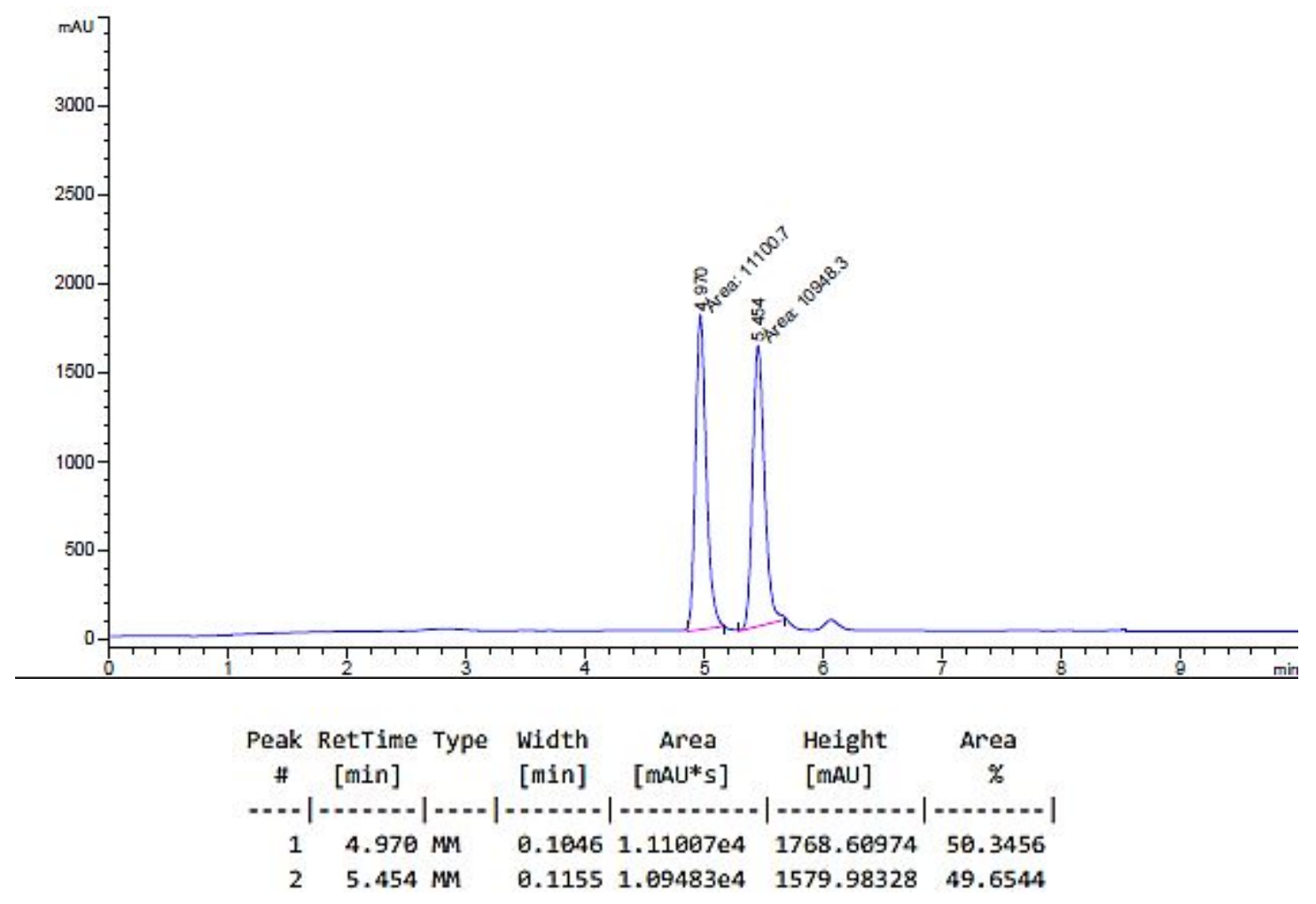

7d-HPLC (94\%) 


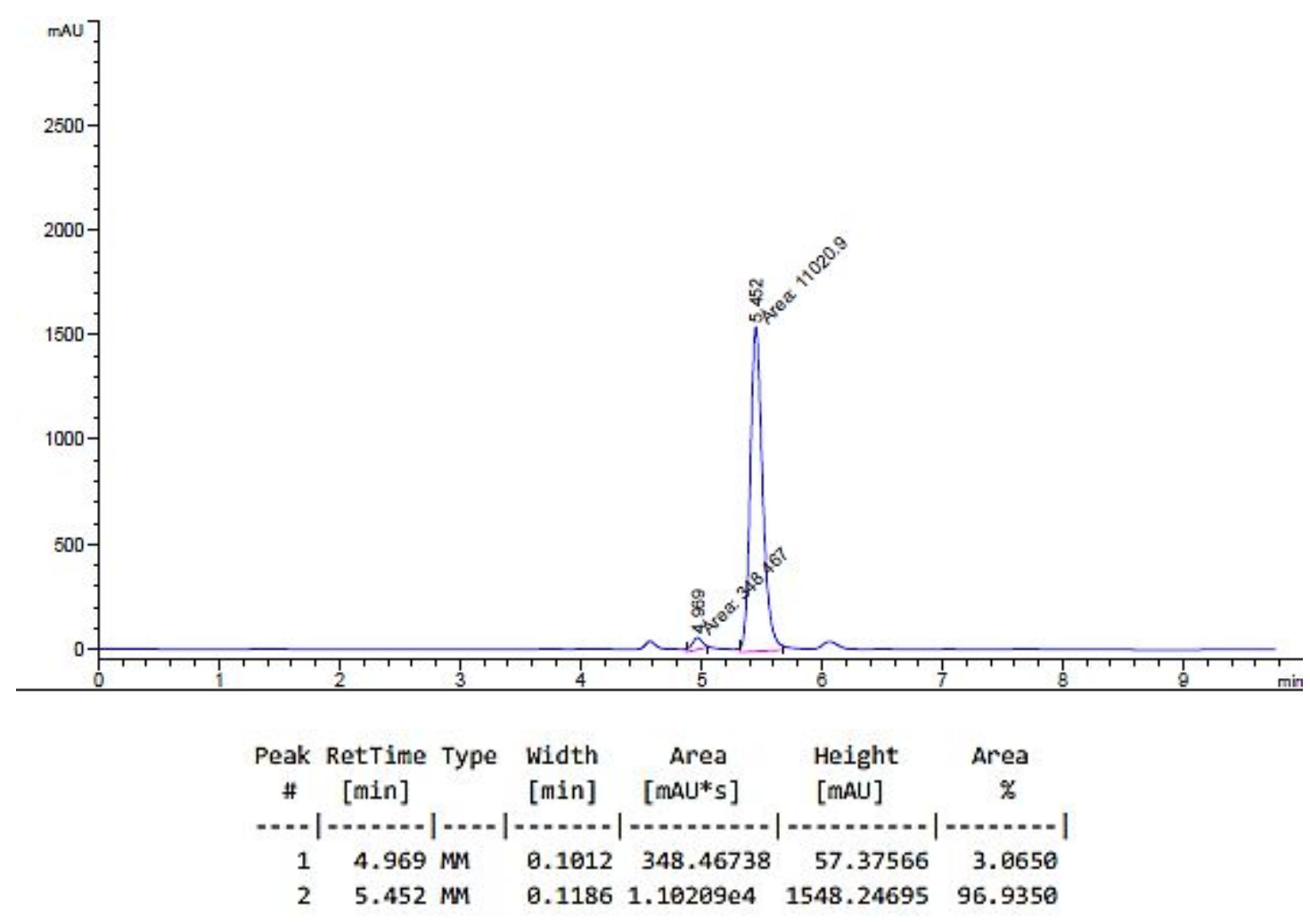

(S,E)-1-(4-bromophenyl)-4-methyl-2-phenylpent-2-en-1-ol (8d) - ${ }^{1} \mathrm{H}$ NMR

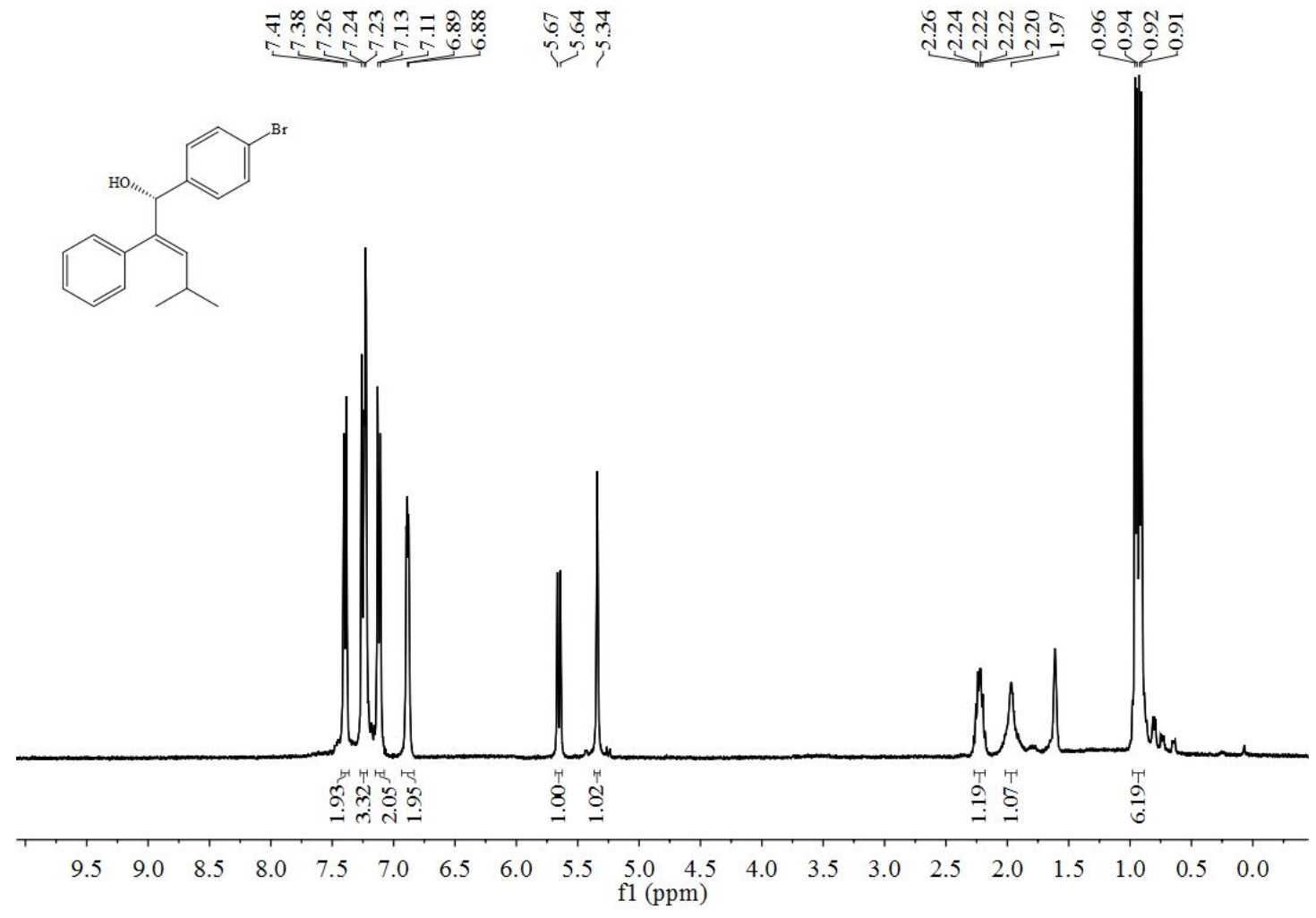

(S,E)-1-(4-bromophenyl)-4-methyl-2-phenylpent-2-en-1-ol (8d) - ${ }^{13}$ C NMR 

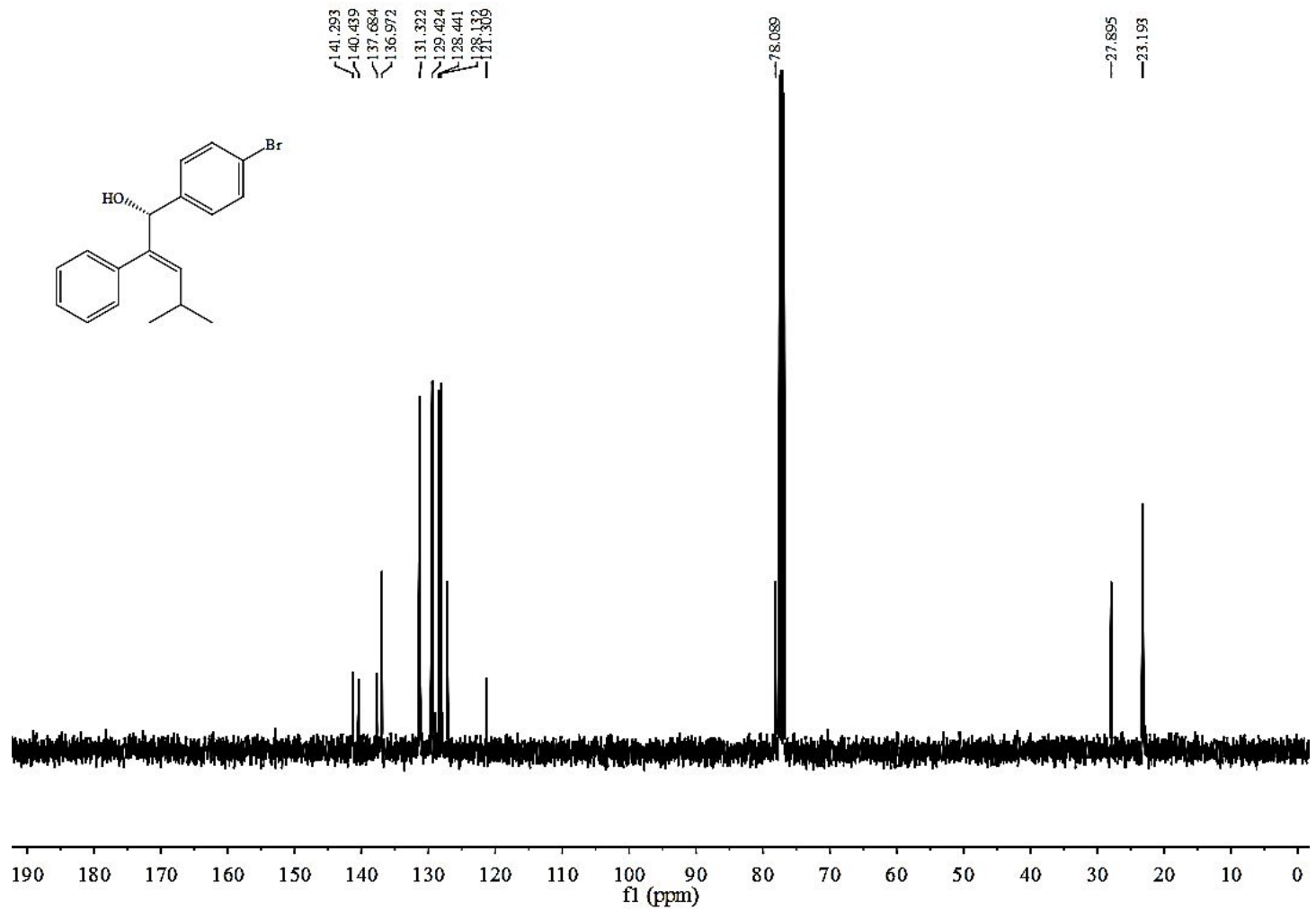

\section{8d-HPLC (racemic)}

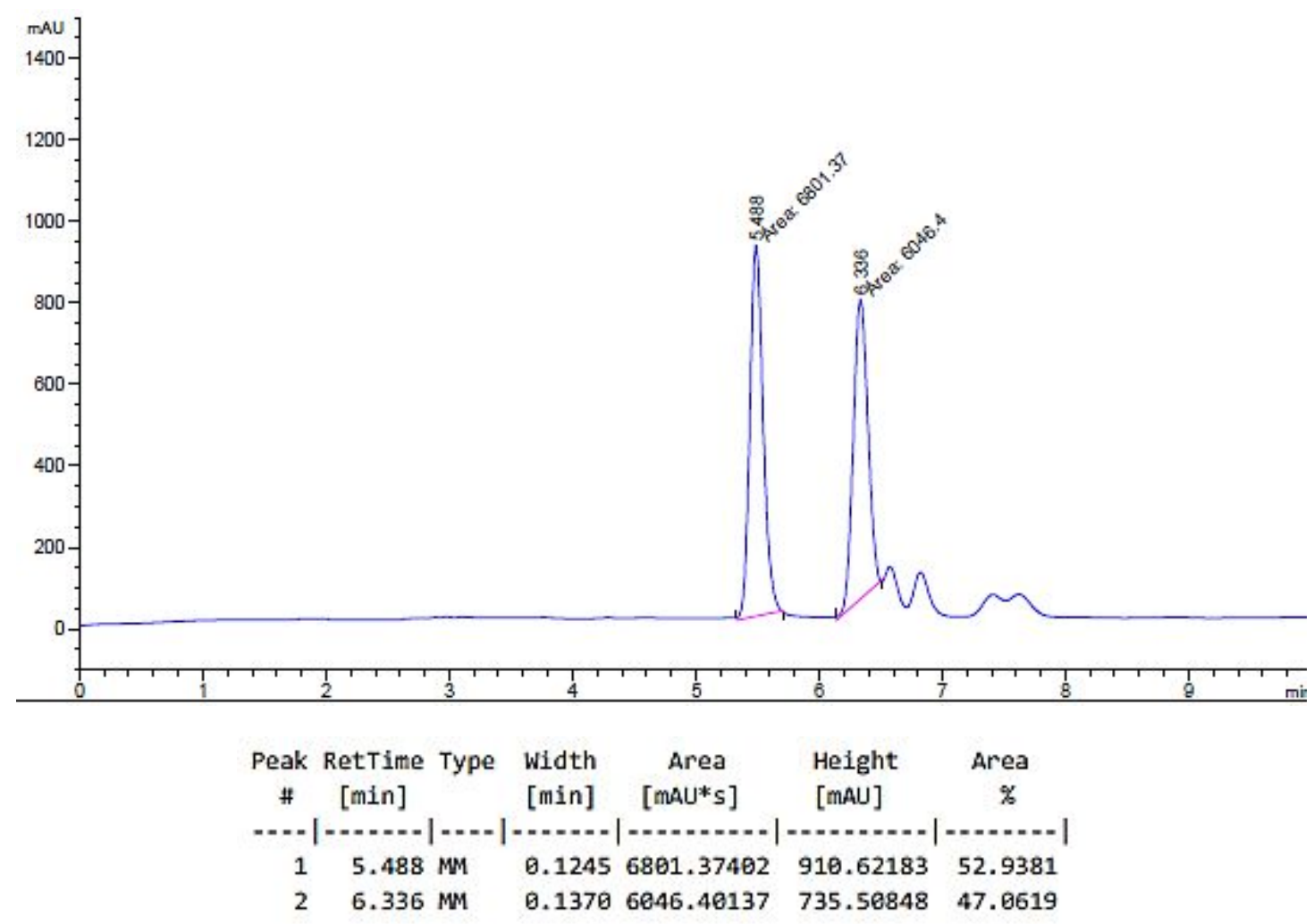

8d-HPLC (96\%) 


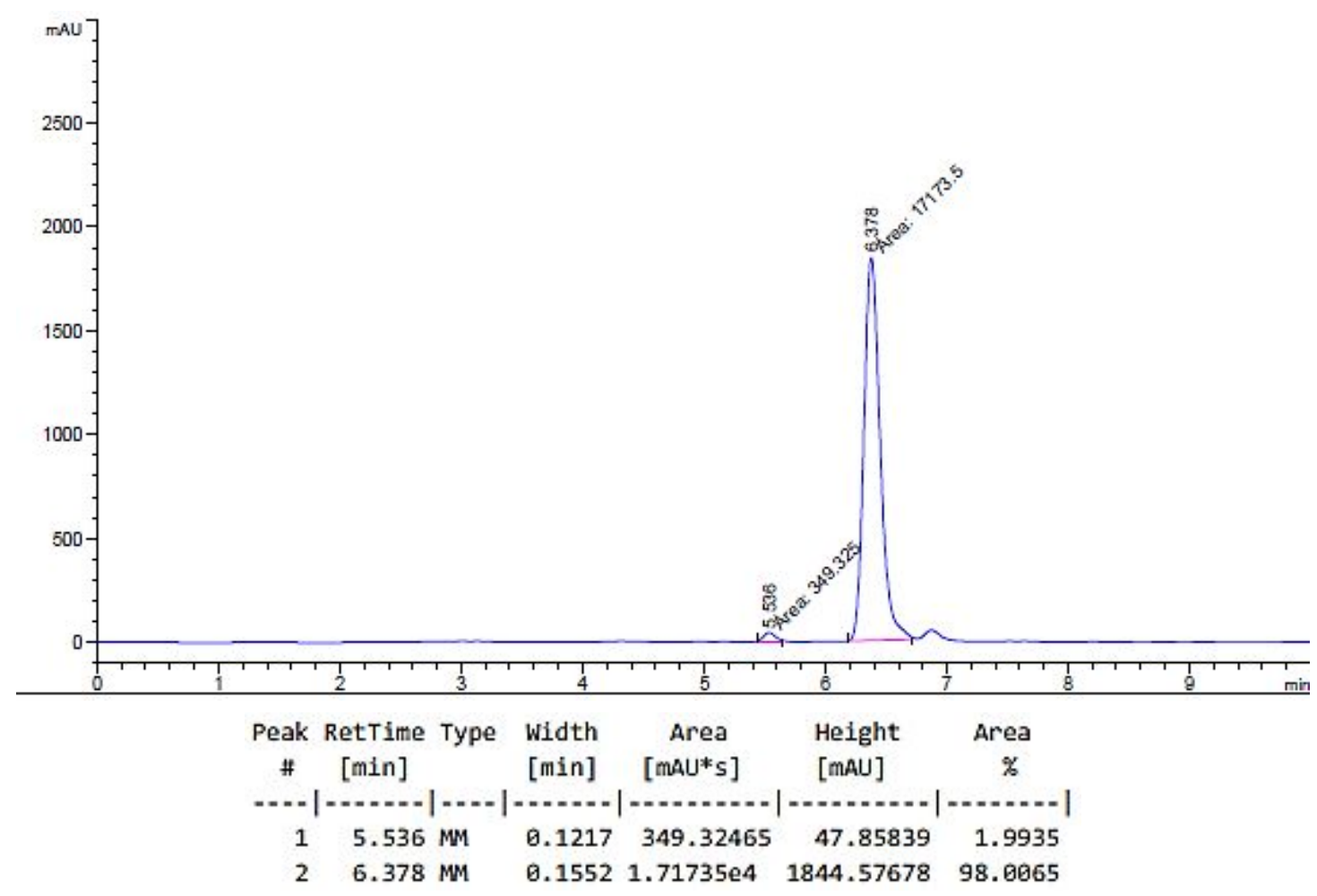

(S,E)-4-methyl-2-phenyl-1-(m-tolyl)pent-2-en-1-ol (9d) - ${ }^{1} \mathrm{H}$ NMR

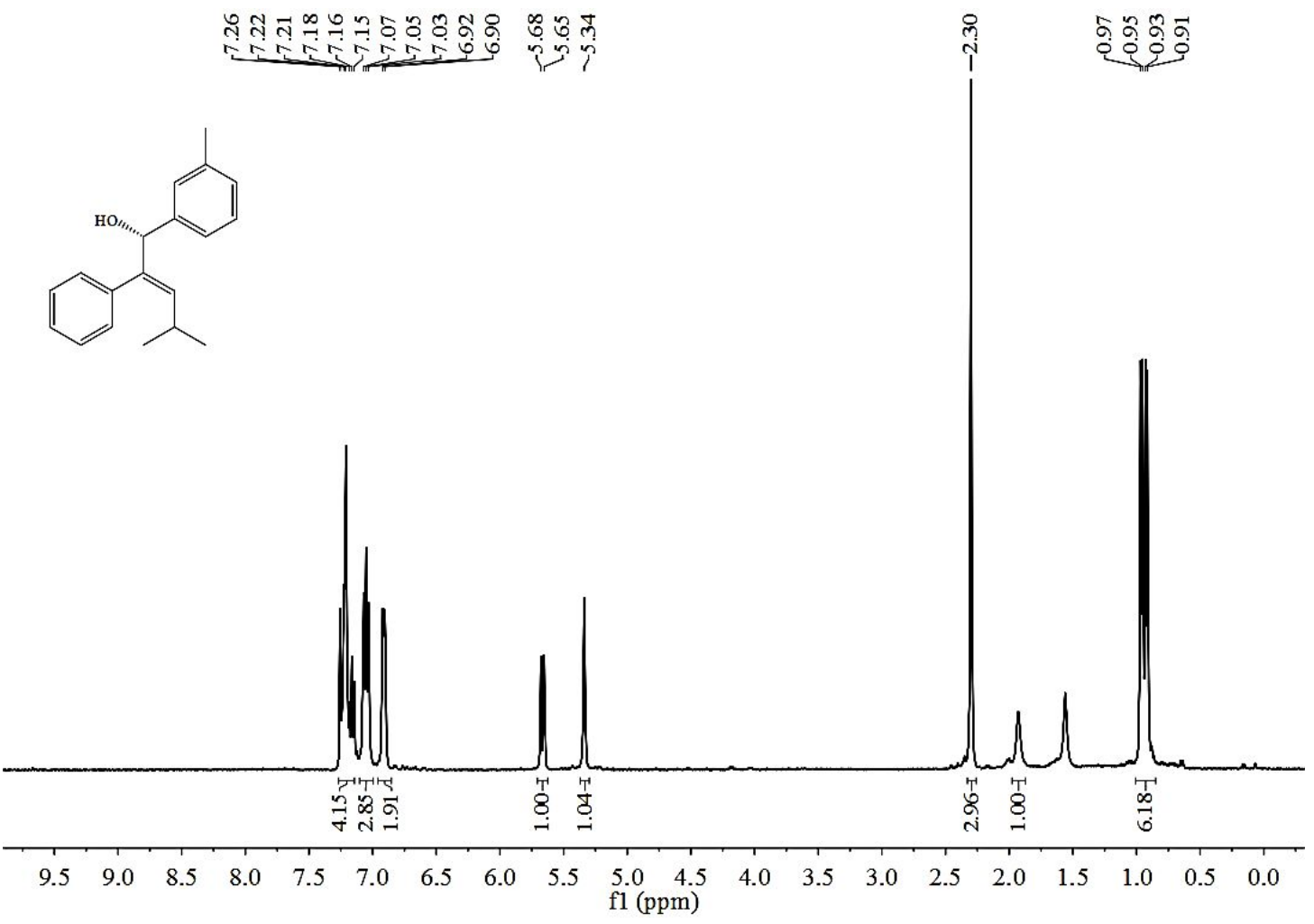

(S,E)-4-methyl-2-phenyl-1-(m-tolyl)pent-2-en-1-ol (9d) - ${ }^{13} \mathrm{C}$ NMR 


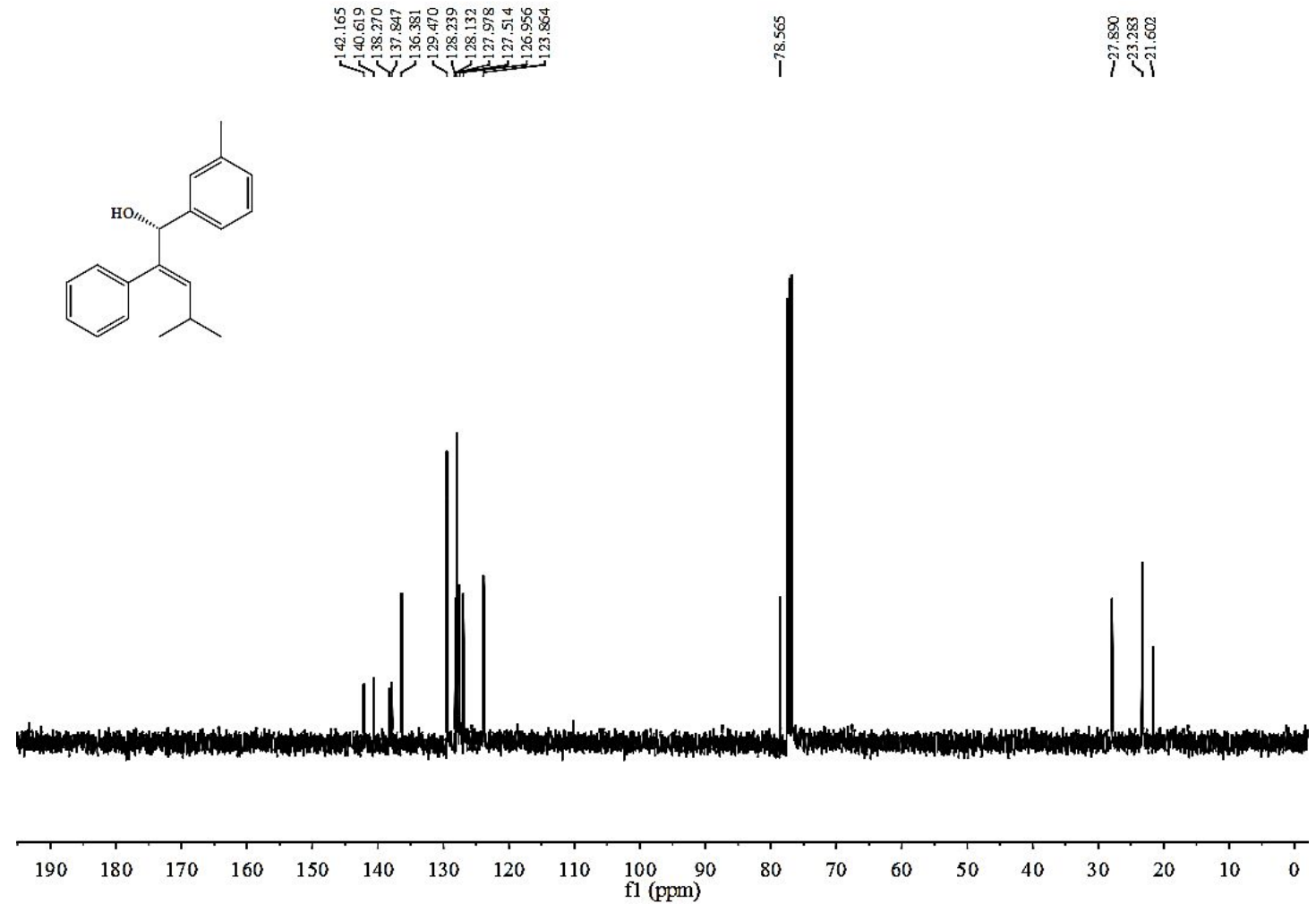

9d-HPLC (racemic)

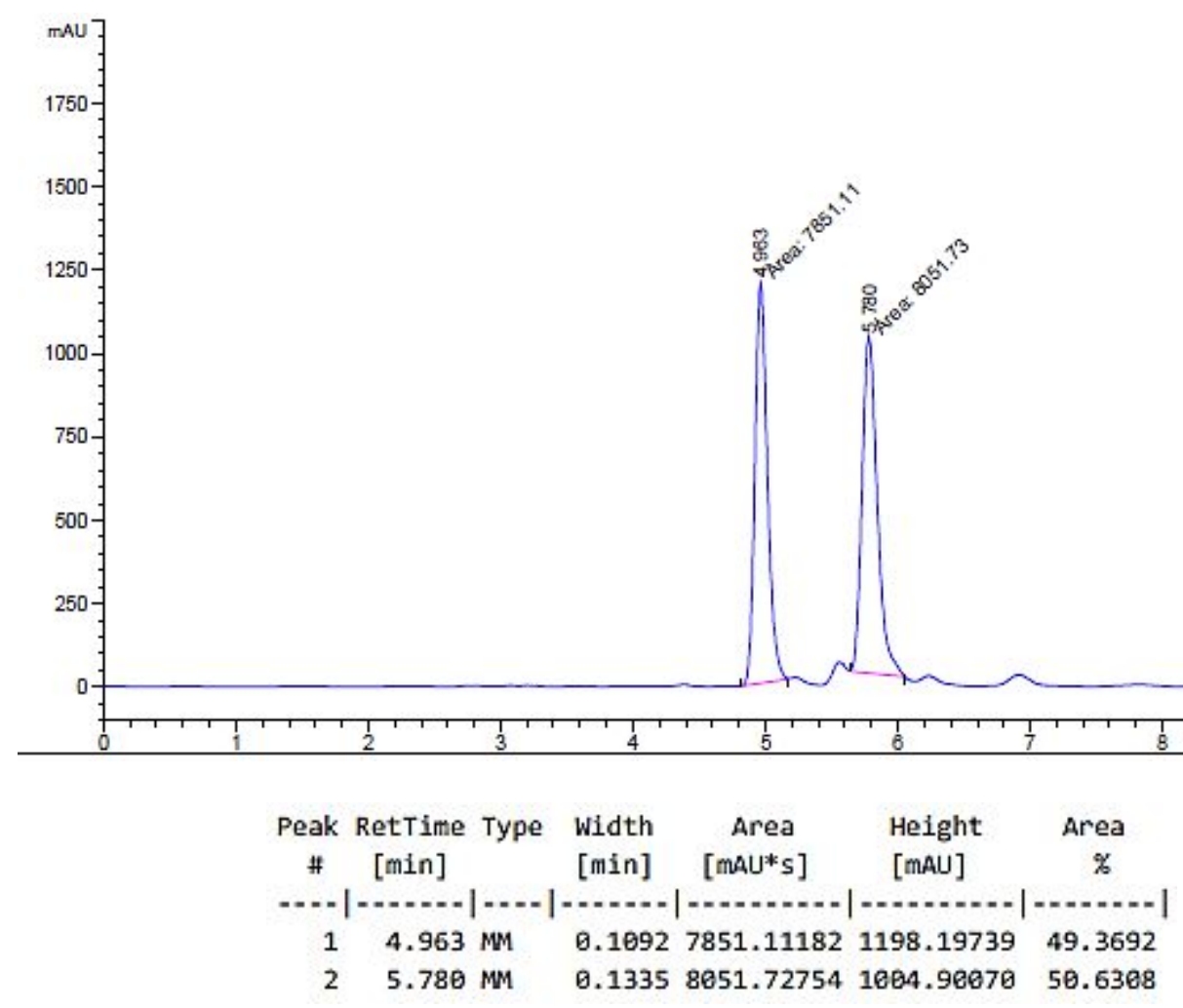

9d-HPLC (95\%) 


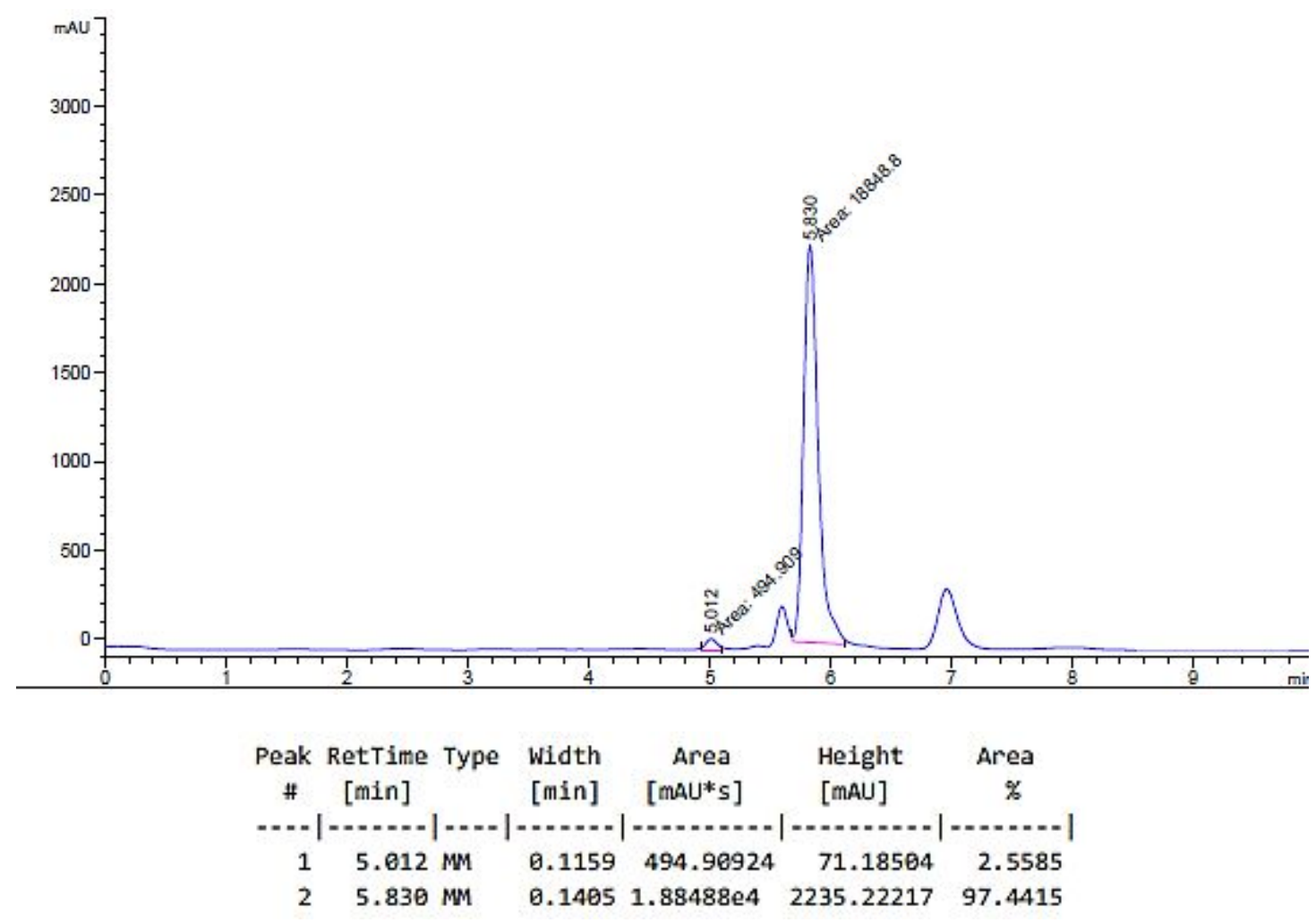

(S,E)-6-methyl-1-phenyl-4-(p-tolyl)hept-4-en-3-ol (10d) - ${ }^{-1} \mathrm{H}$ NMR

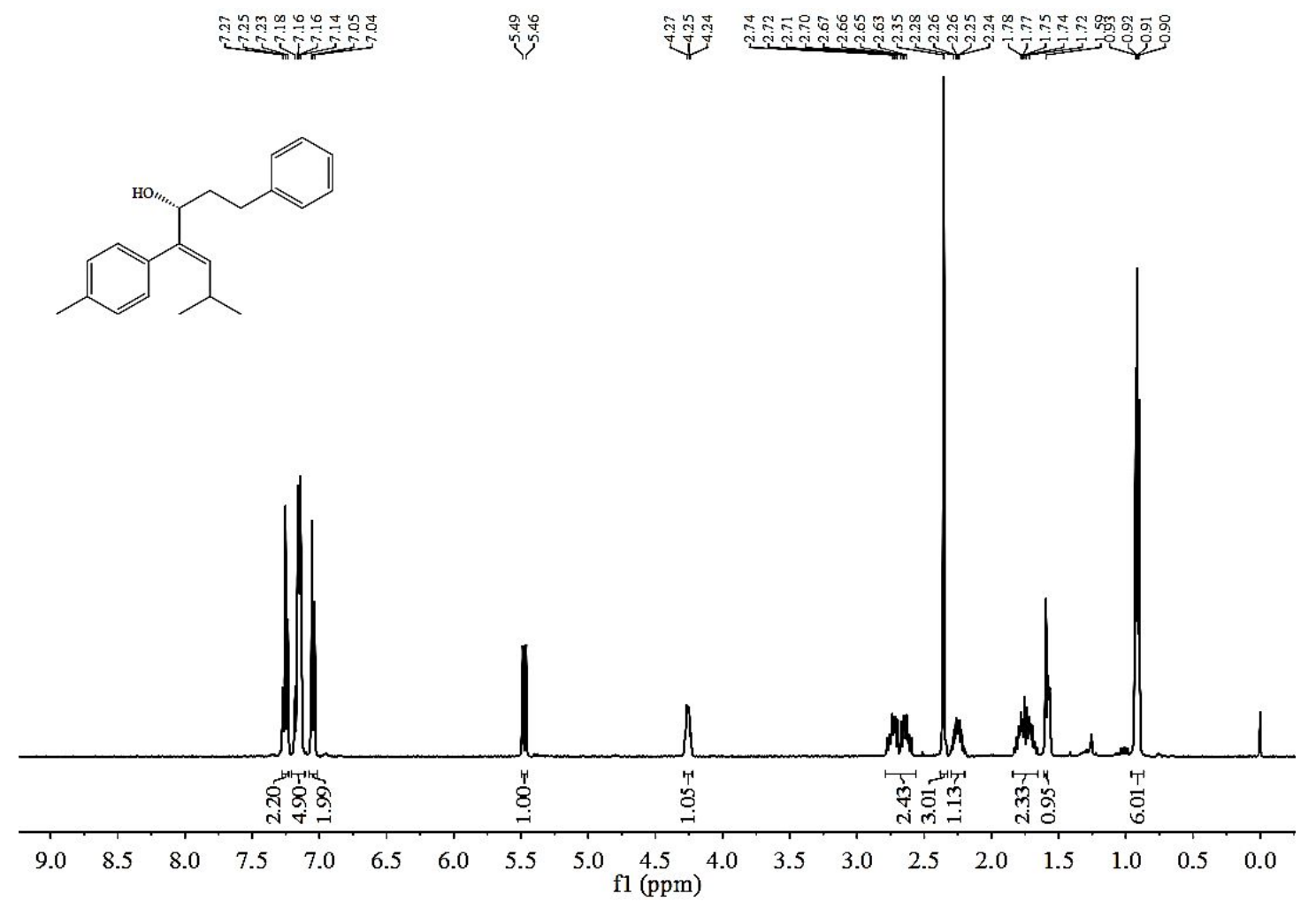

(S,E)-6-methyl-1-phenyl-4-(p-tolyl)hept-4-en-3-ol (10d) - ${ }^{13}$ C NMR 


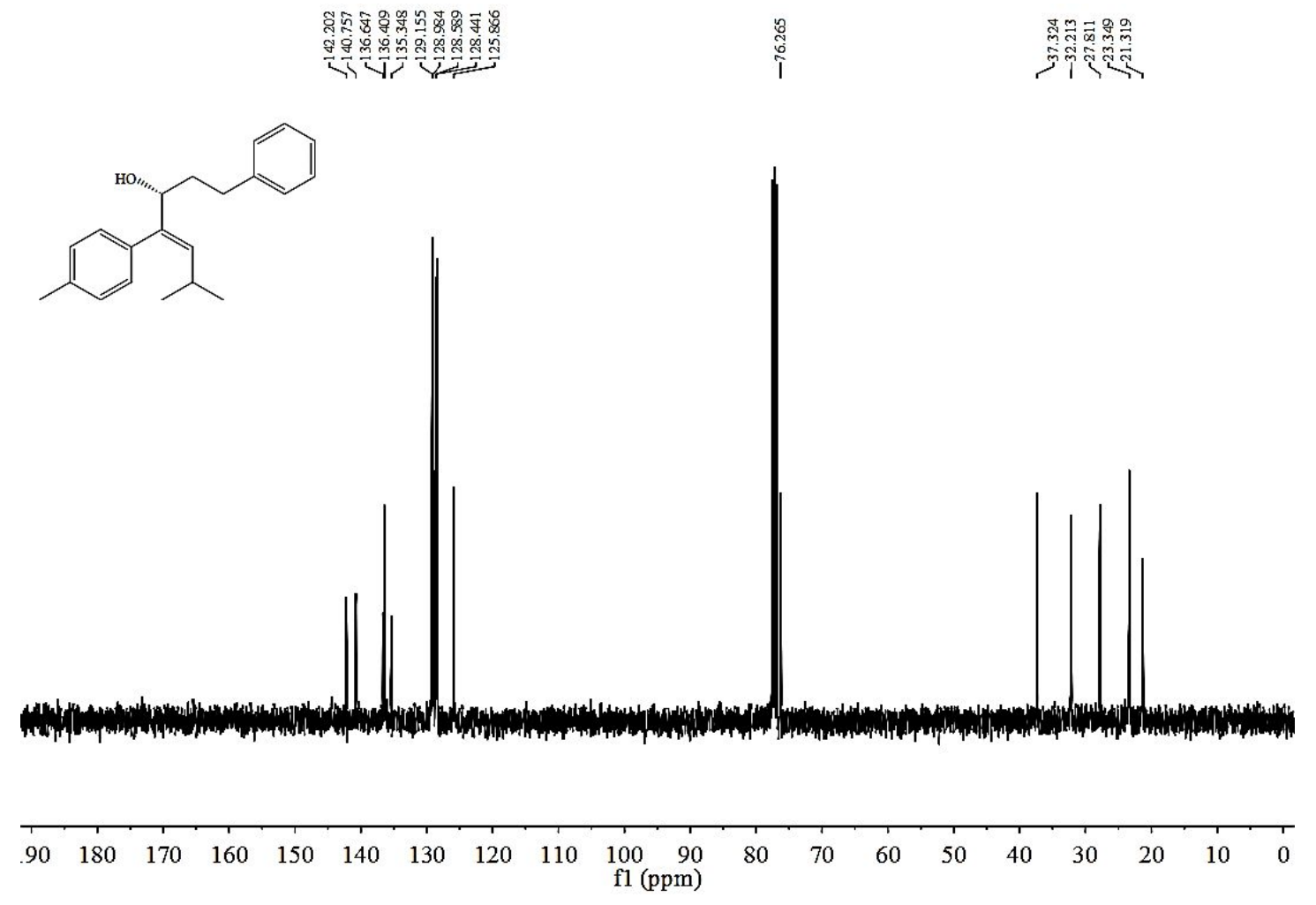

10d-HPLC (racemic)

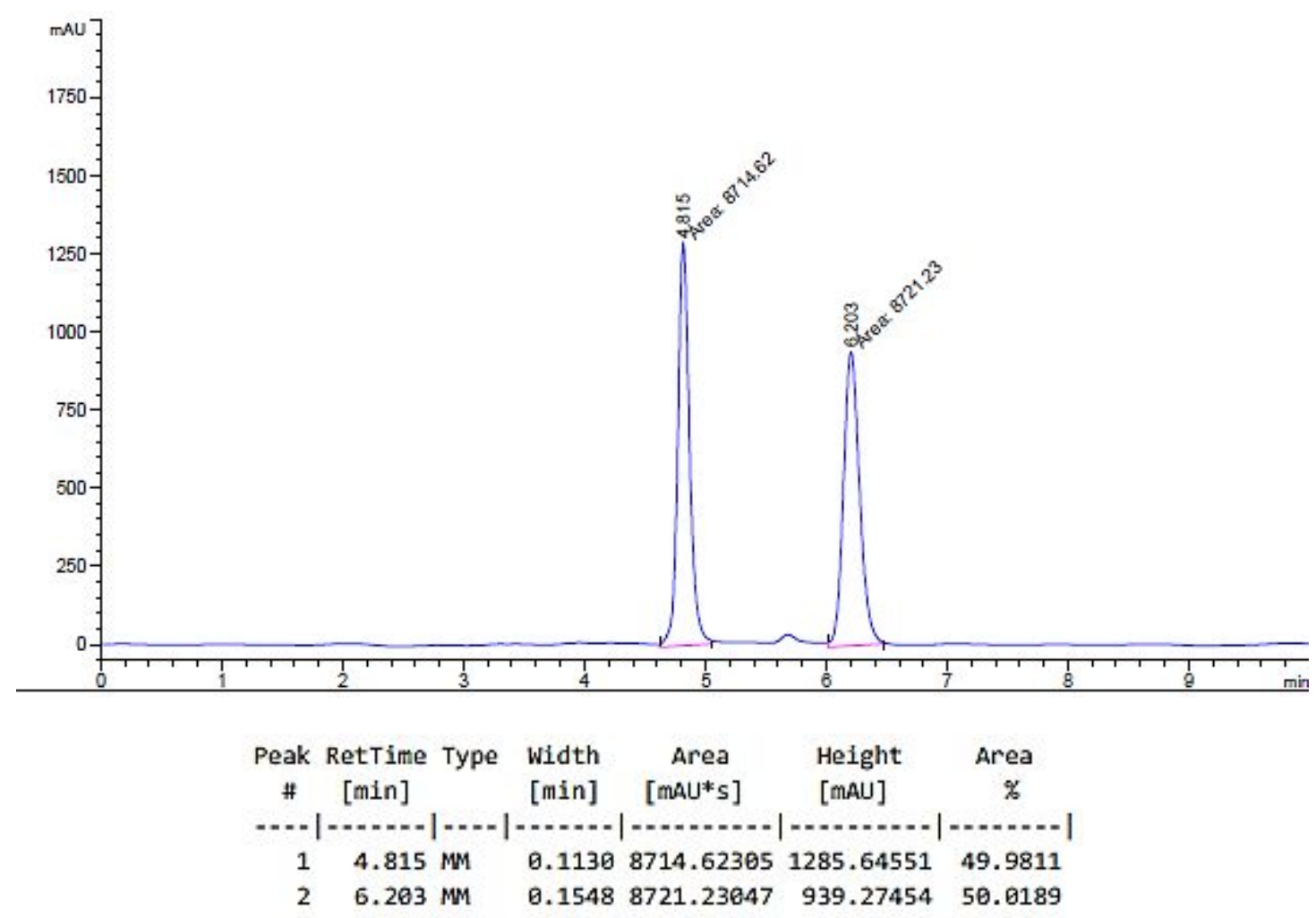

10d-HPLC (91\%) 


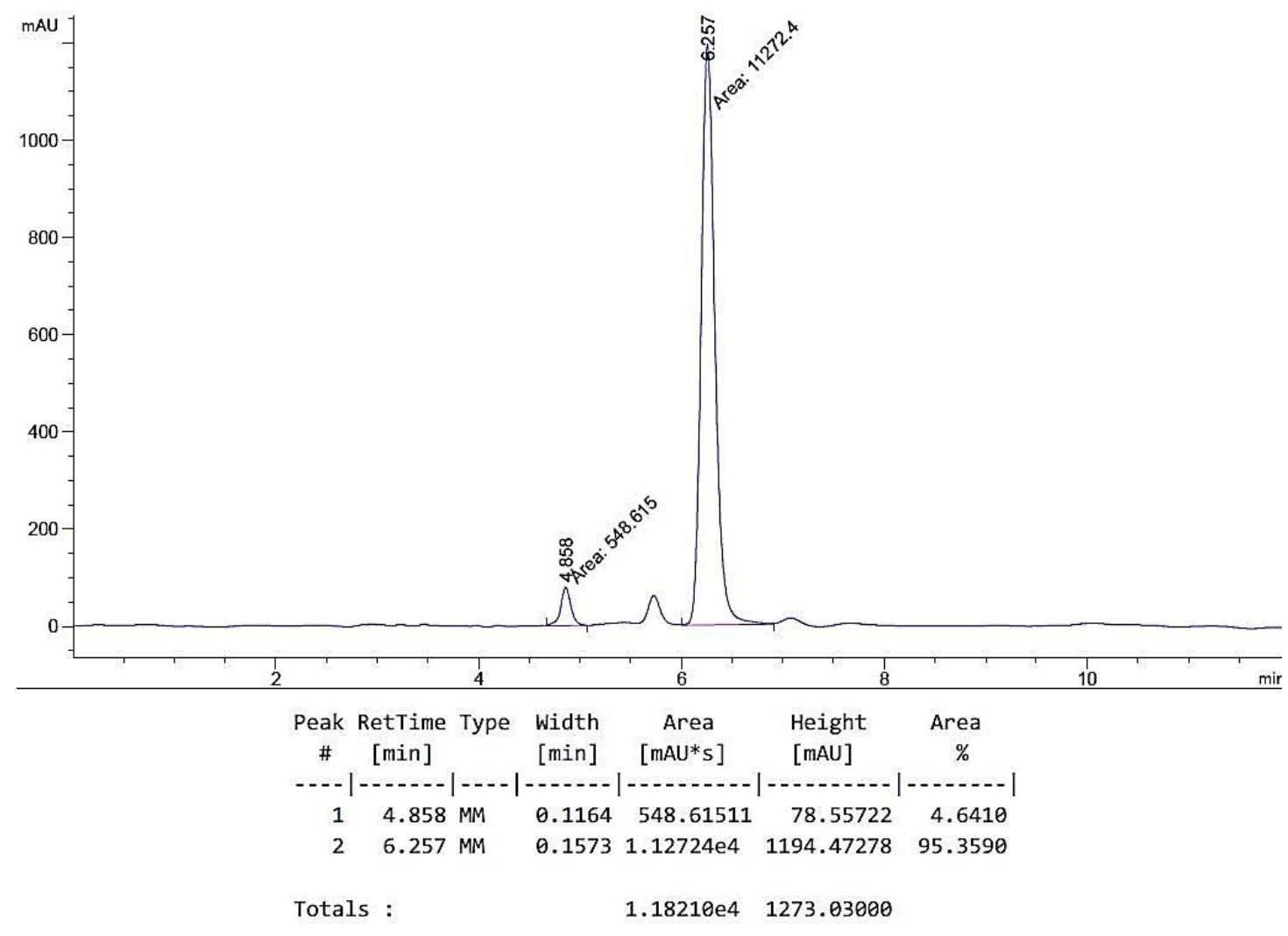

(S,E)-4-(4-methoxyphenyl)-6-methyl-1-phenylhept-4-en-3-ol (11d) - ${ }^{1} \mathrm{H}$ NMR

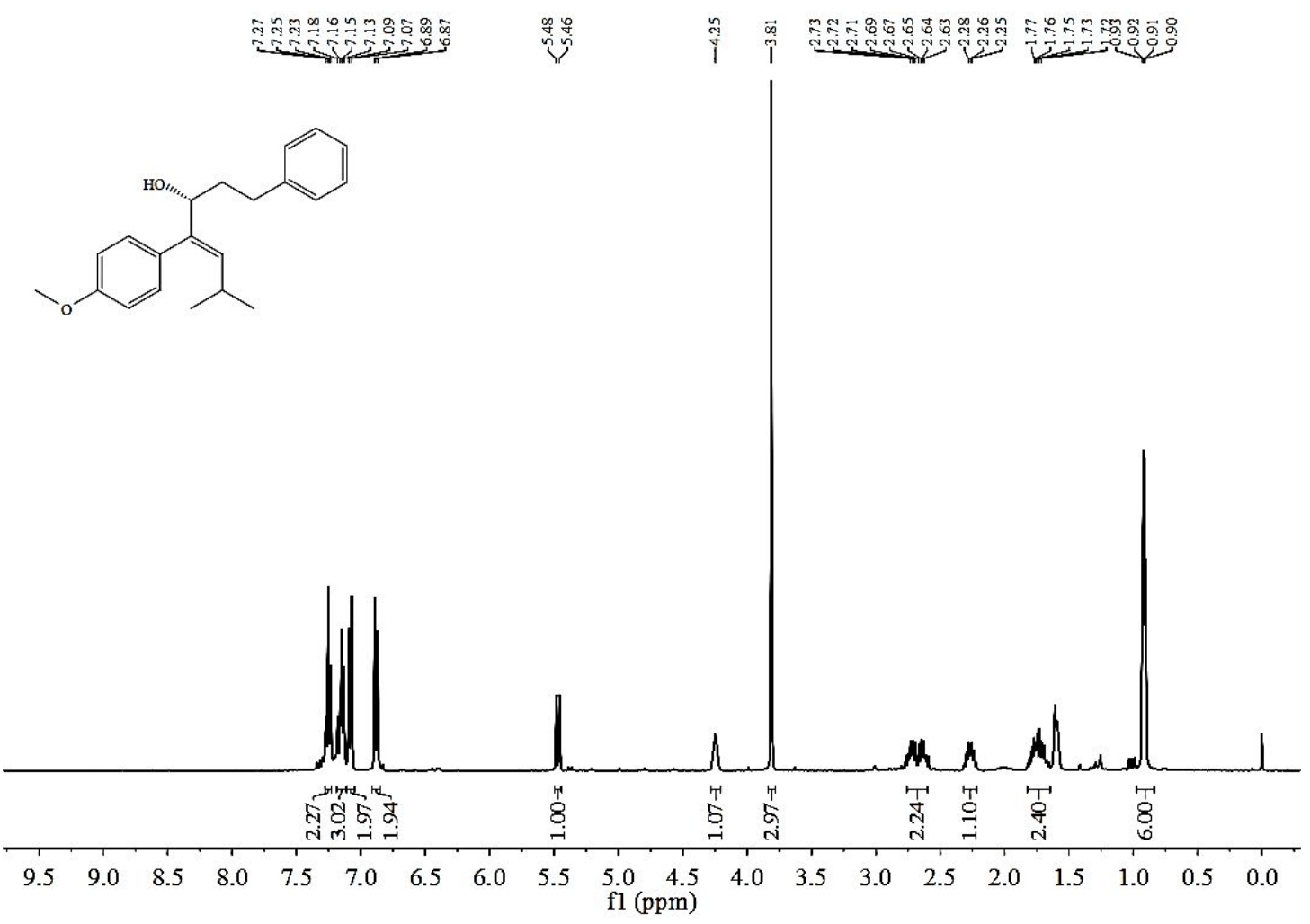

(S,E)-4-(4-methoxyphenyl)-6-methyl-1-phenylhept-4-en-3-ol (11d) - ${ }^{13} \mathrm{C}$ NMR 


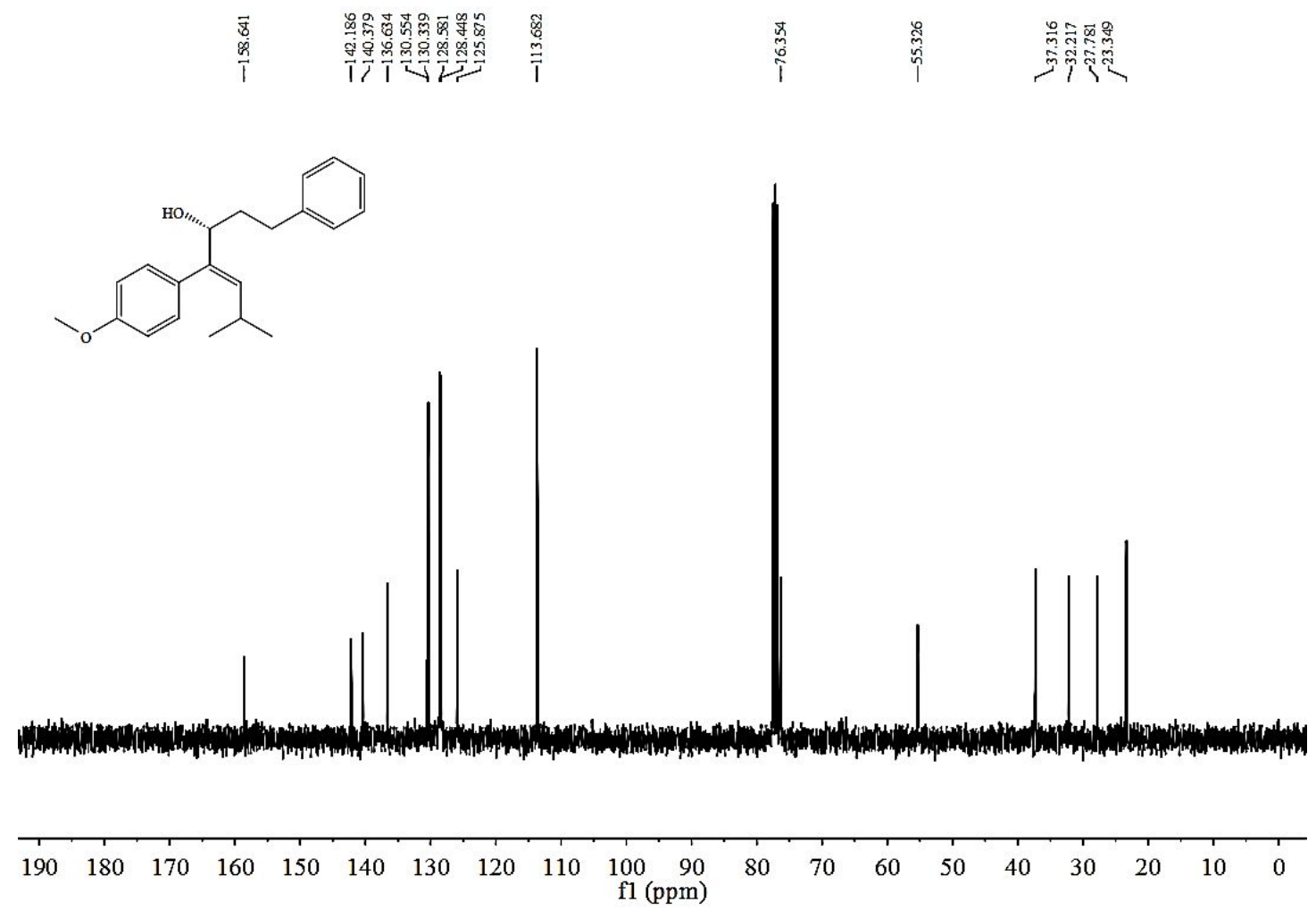

11d-HPLC (racemic)

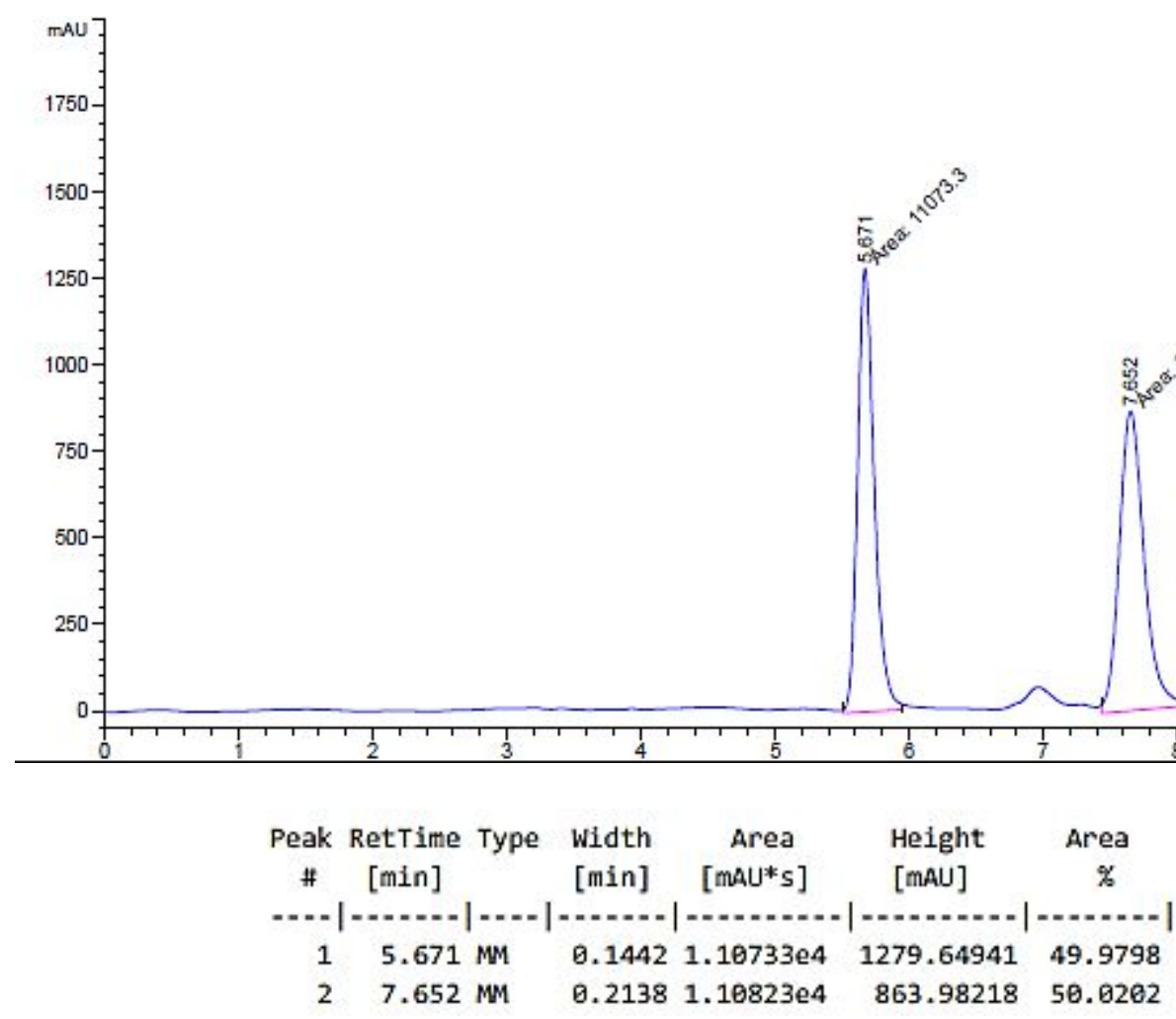

11d-HPLC (92\%) 


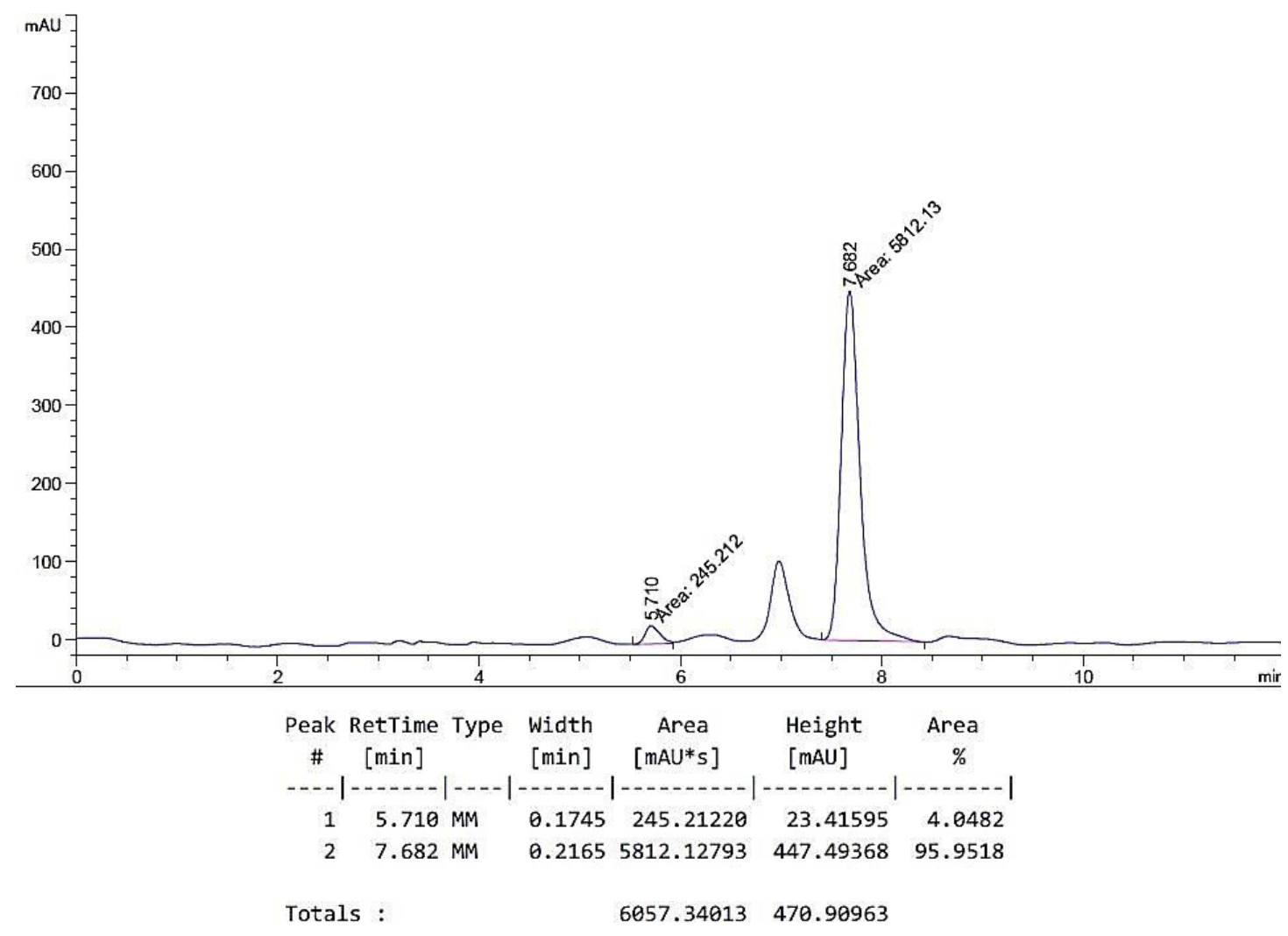

(S,E)-4-(4-(tert-butyl)phenyl)-6-methyl-1-phenylhept-4-en-3-ol (12d) - ${ }^{1} \mathrm{H}$ NMR

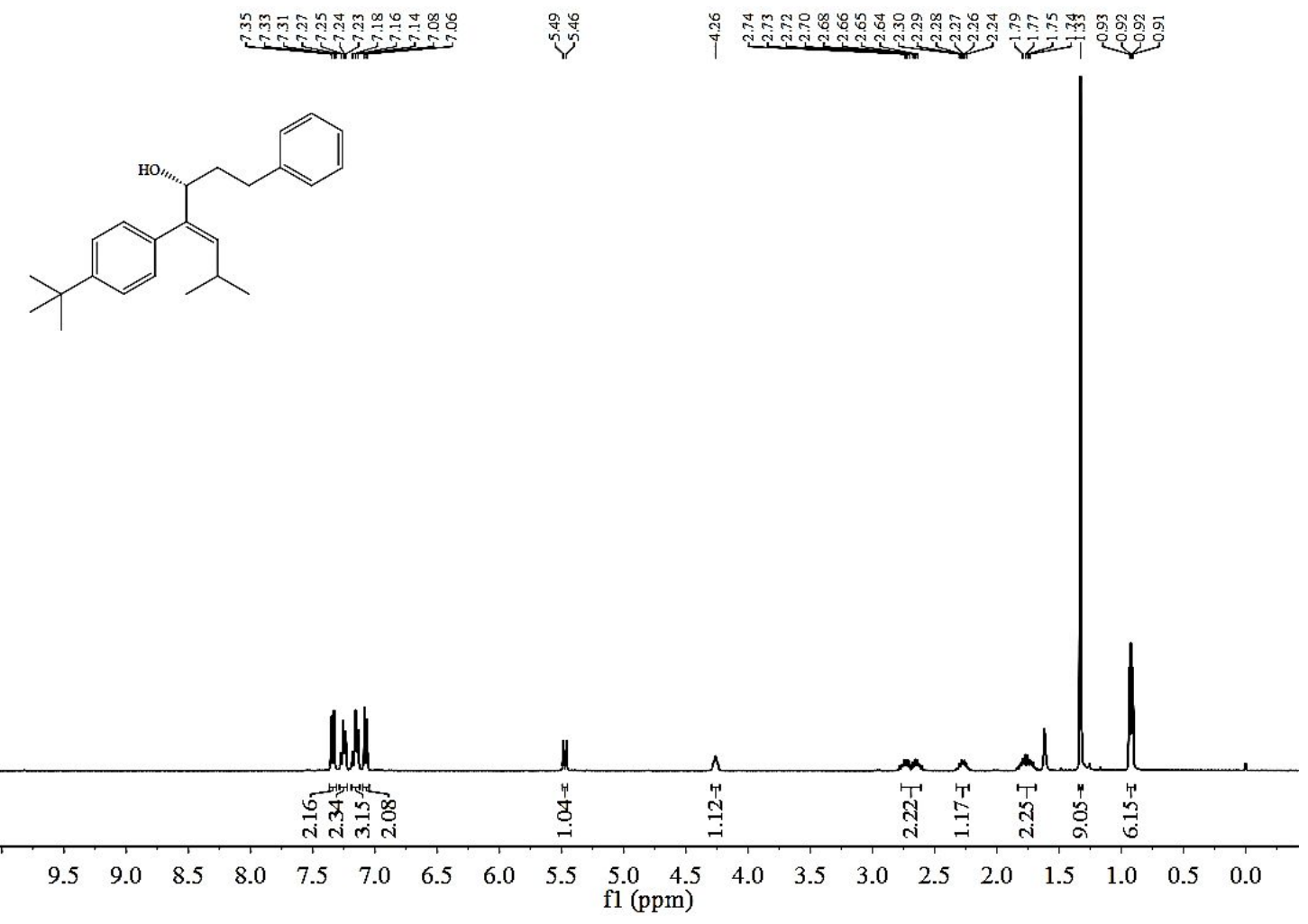

(S,E)-4-(4-(tert-butyl)phenyl)-6-methyl-1-phenylhept-4-en-3-ol (12d) - ${ }^{13} \mathrm{C}$ NMR 

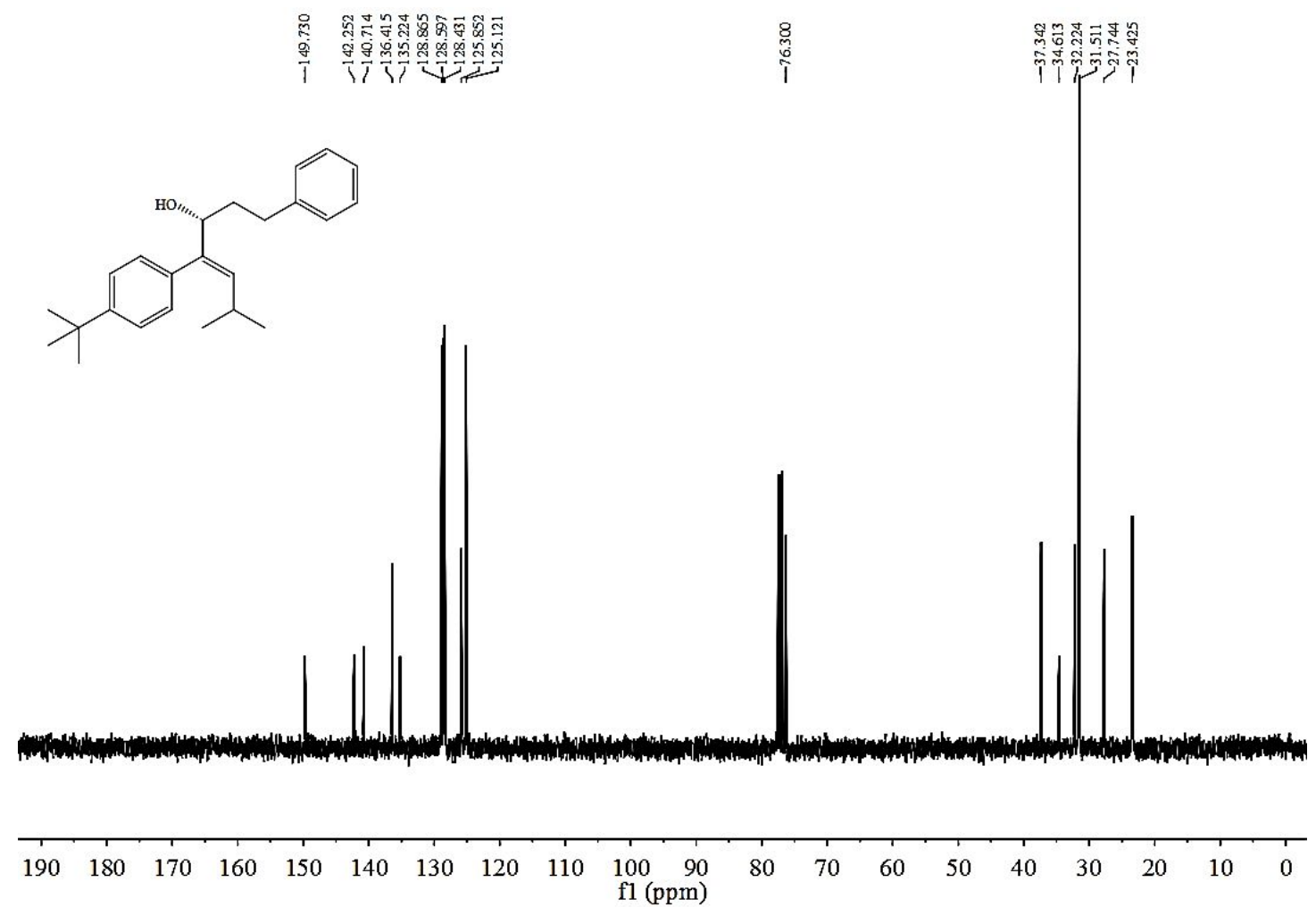

\section{2d-HPLC (racemic)}

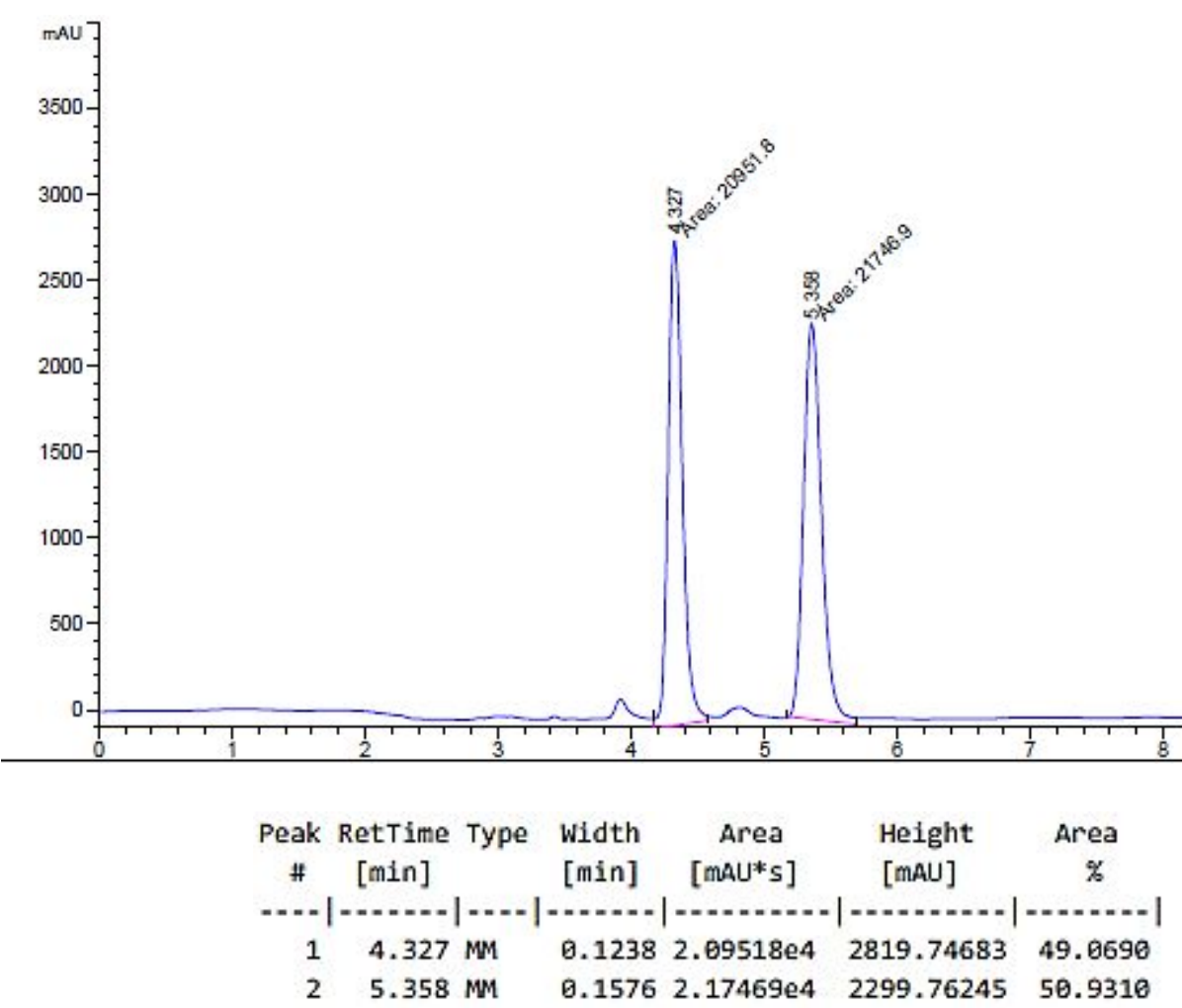

12d-HPLC (91\%) 


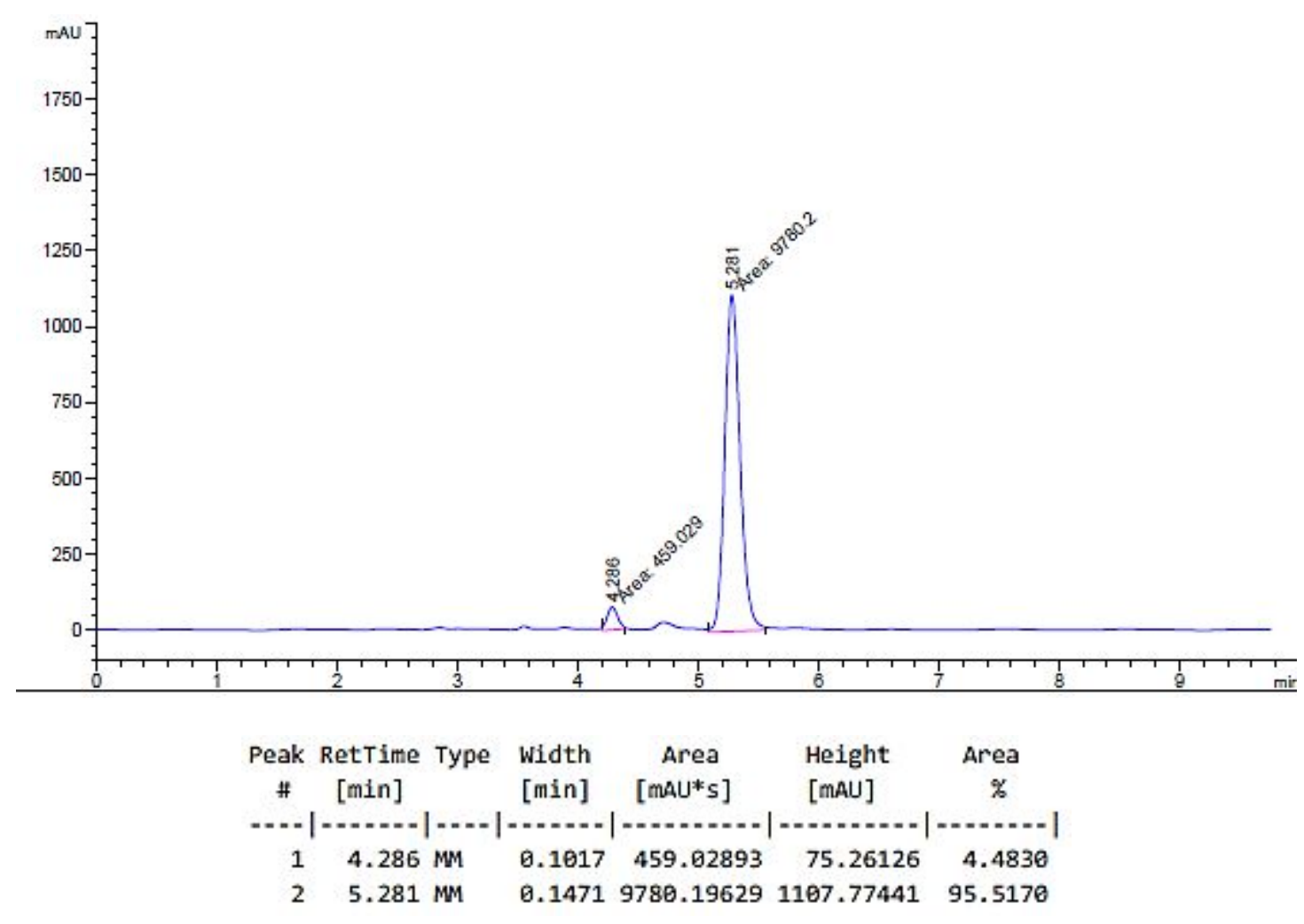

(S,E)-4-(3-chlorophenyl)-6-methyl-1-phenylhept-4-en-3-ol (13d) - ${ }^{1}$ H NMR
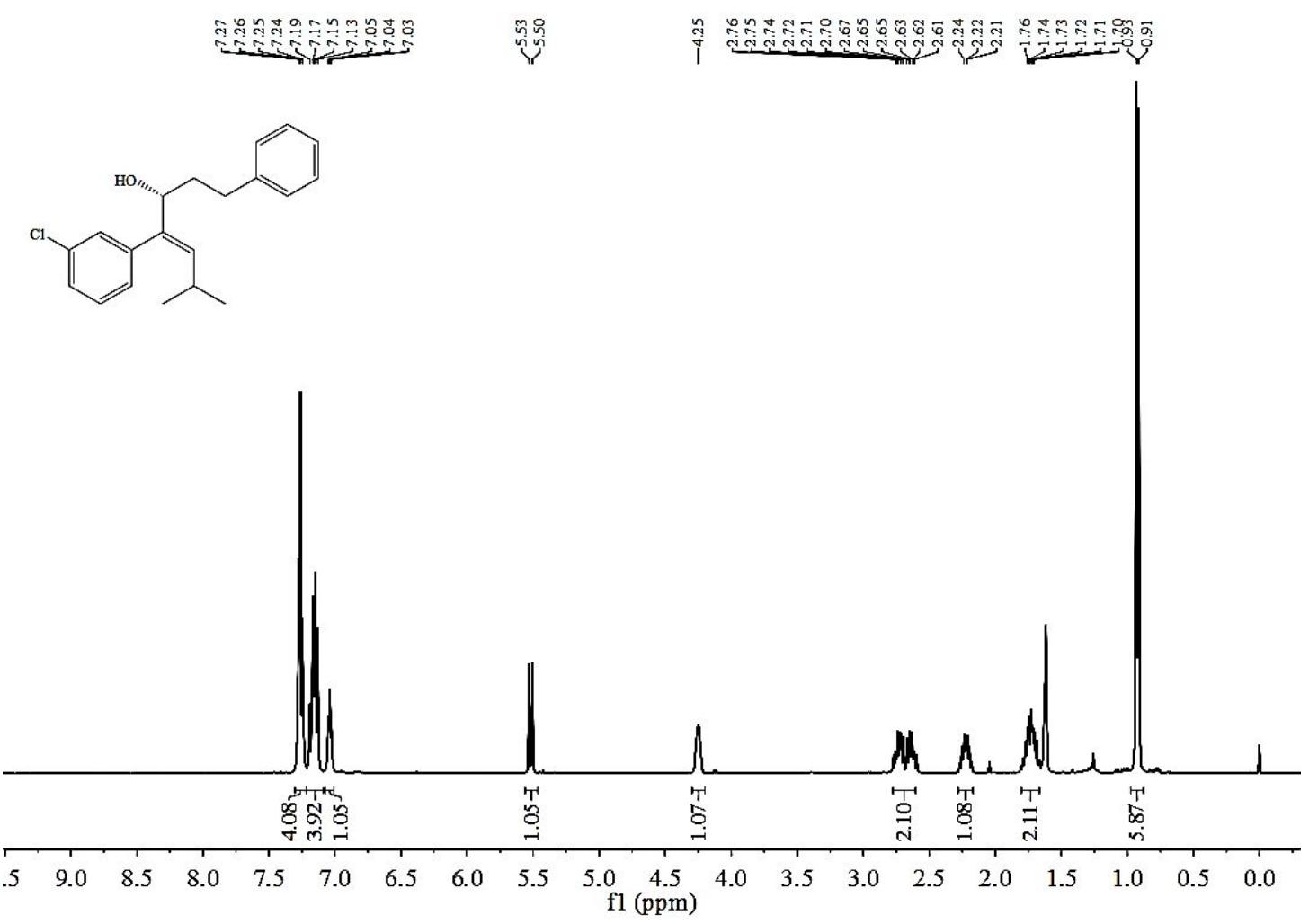

(S,E)-4-(3-chlorophenyl)-6-methyl-1-phenylhept-4-en-3-ol (13d) $-{ }^{-13} \mathrm{C}$ NMR 

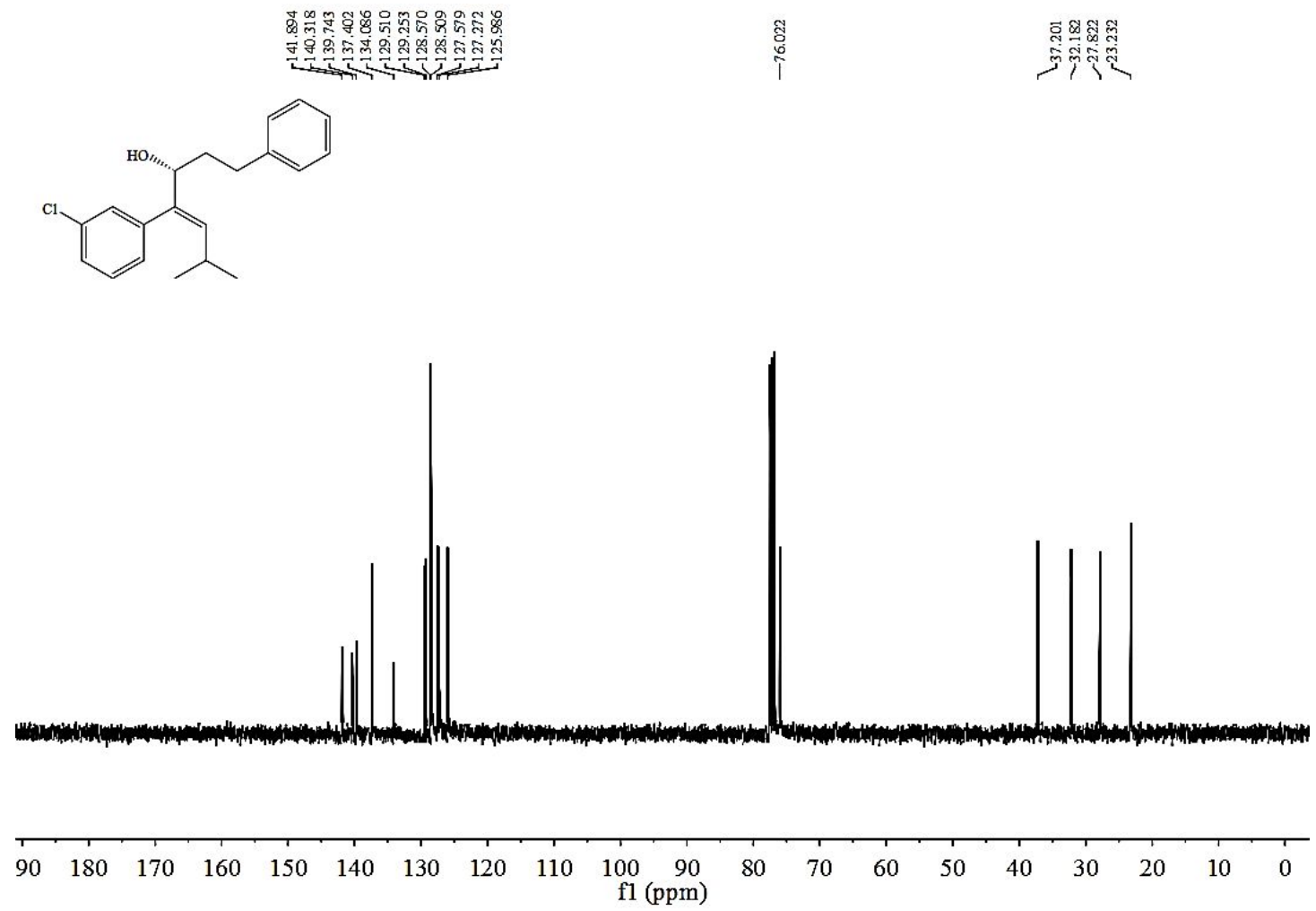

\section{3d-HPLC (racemic)}

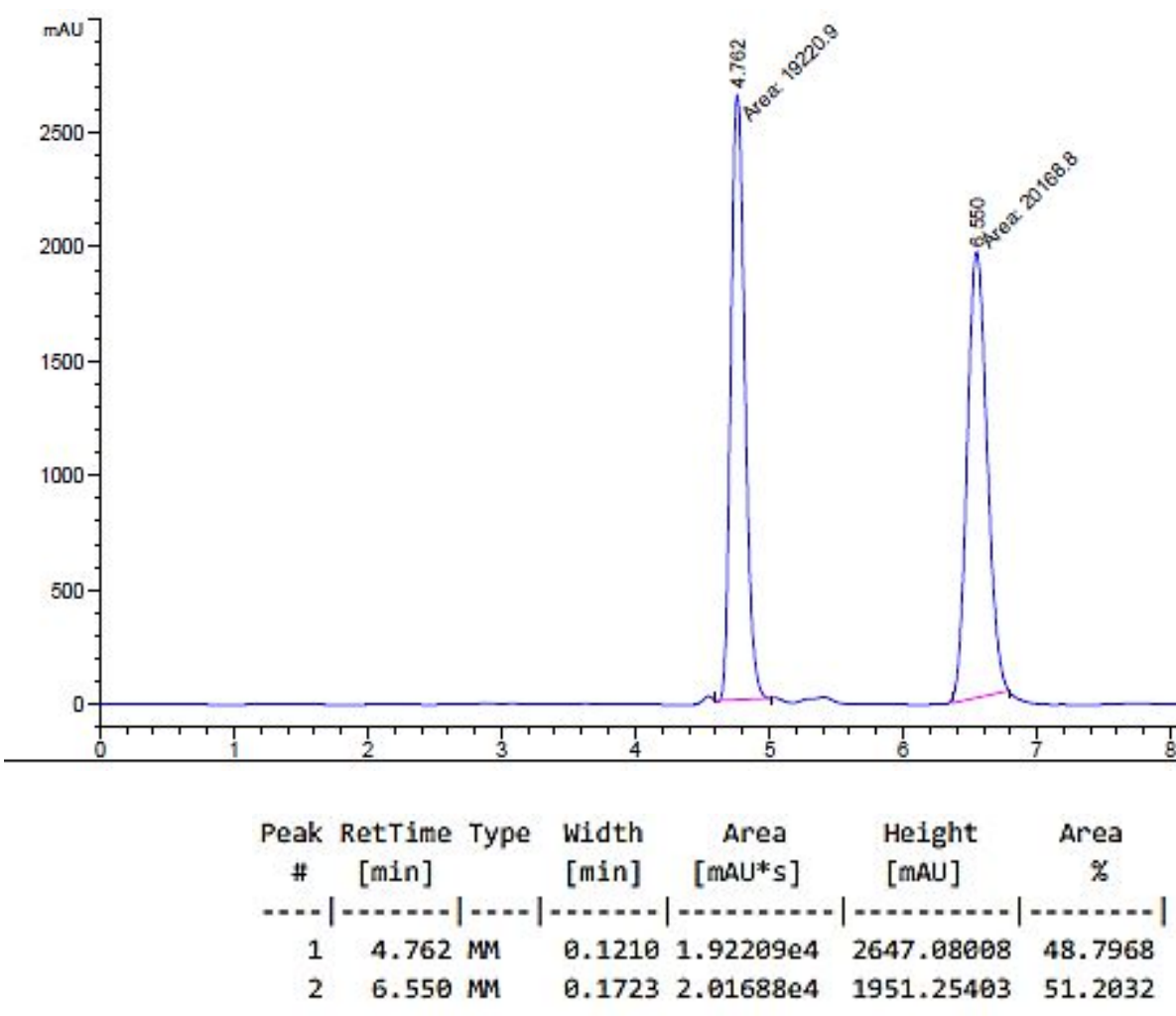

13d-HPLC (95\%) 


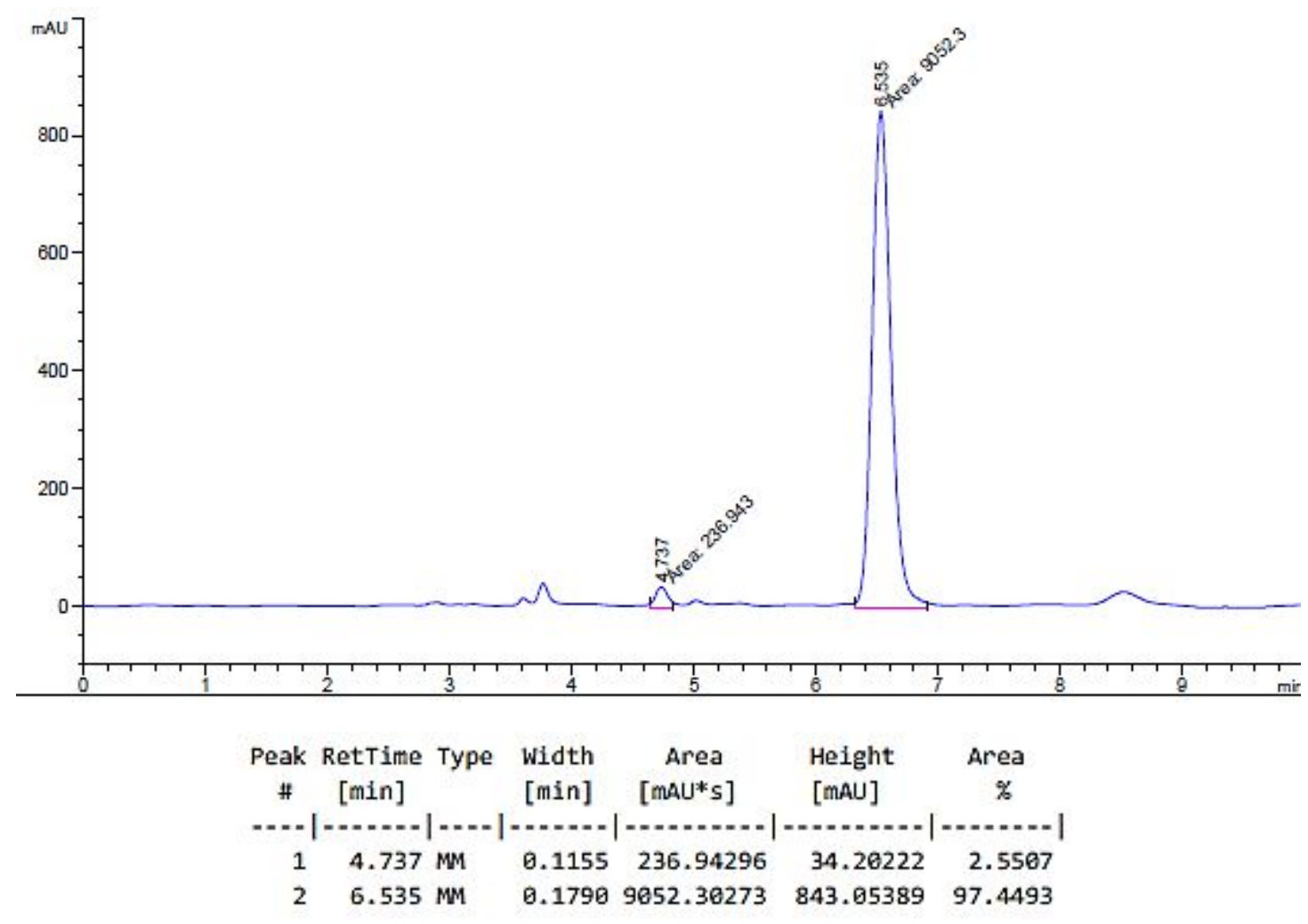

(S,E)-4-(4-bromophenyl)-6-methyl-1-phenylhept-4-en-3-ol (14d) - ${ }^{1} \mathrm{H}$ NMR

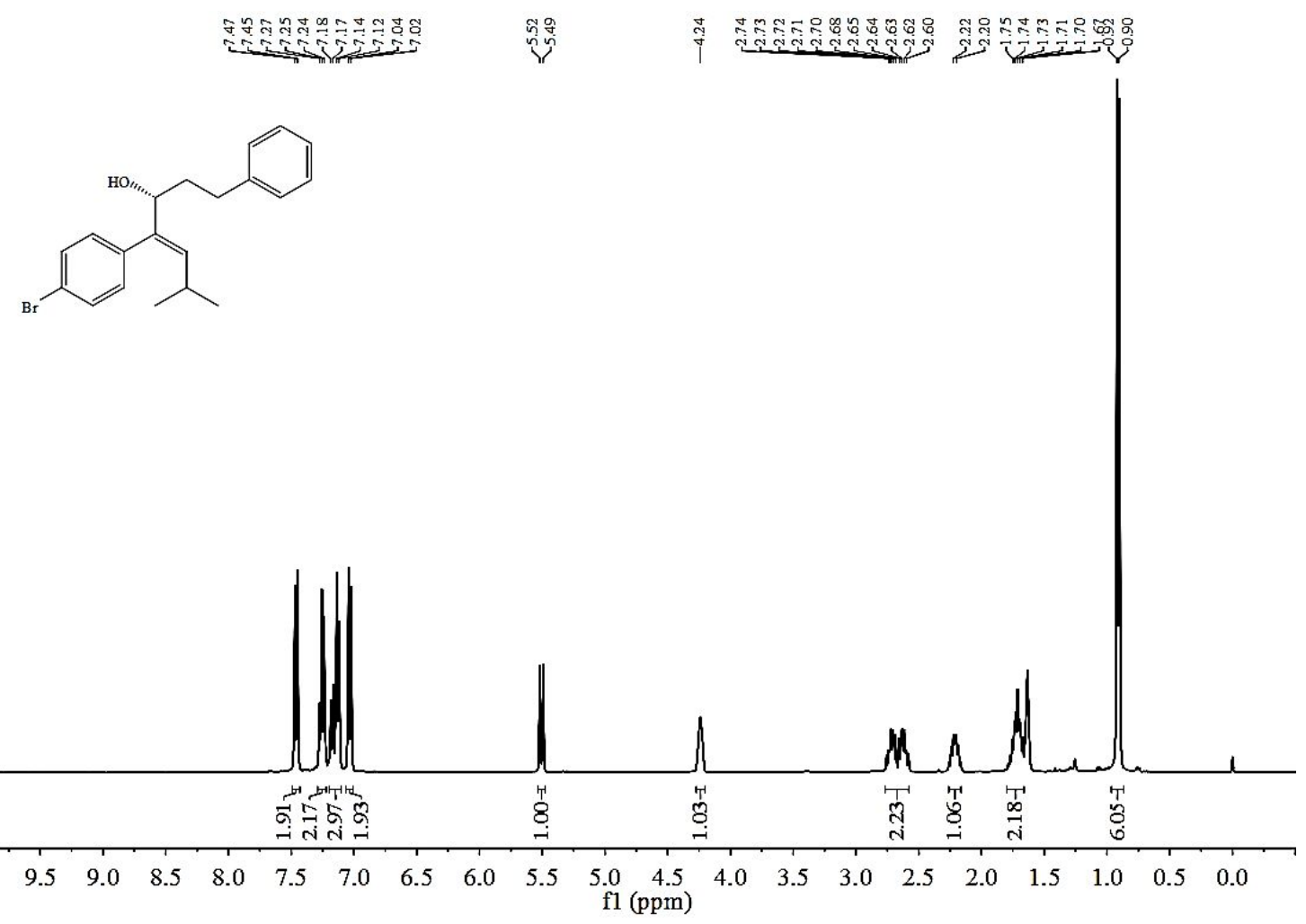

(S,E)-4-(4-bromophenyl)-6-methyl-1-phenylhept-4-en-3-ol (14d) - ${ }^{13} \mathrm{C}$ NMR 

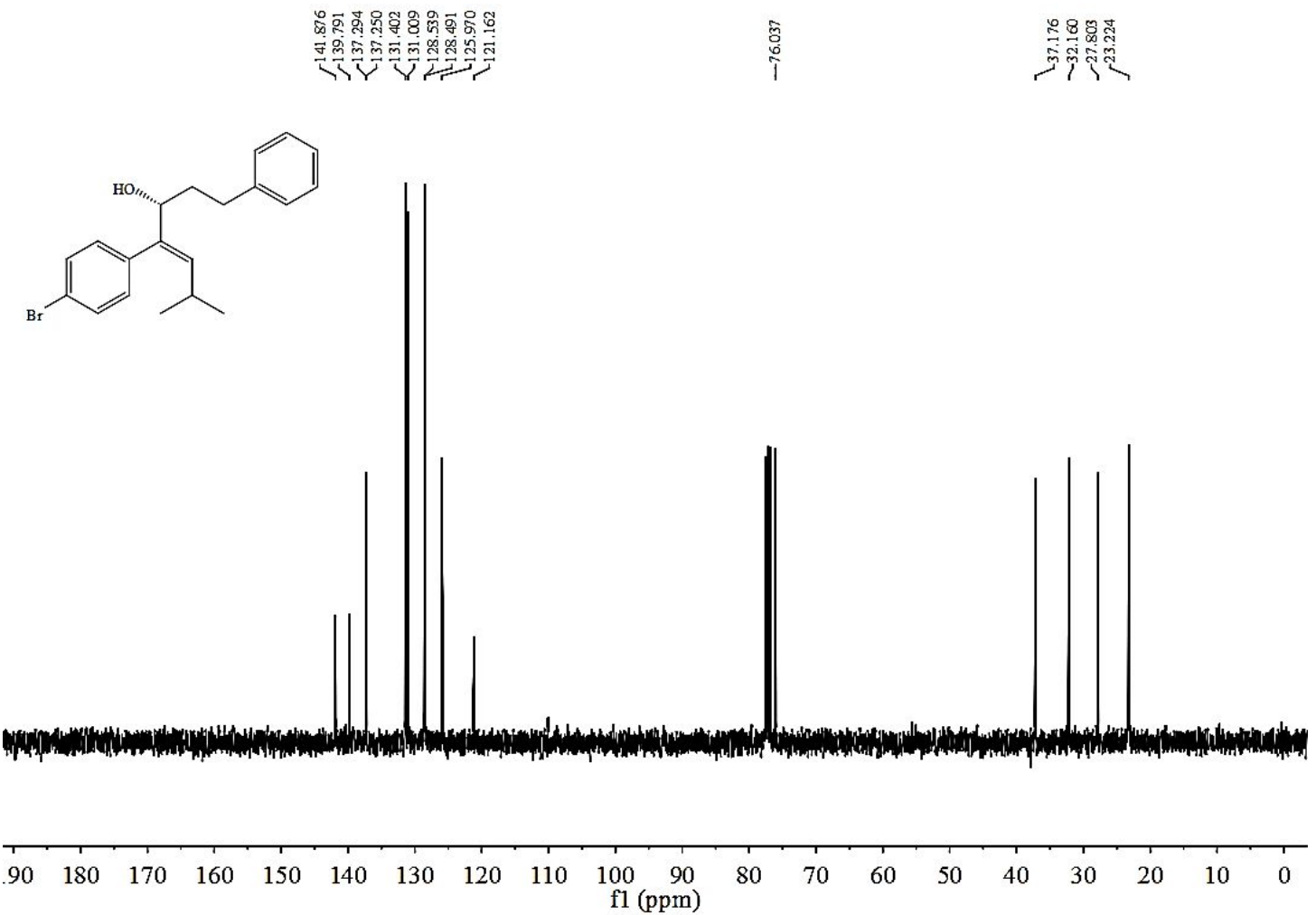

14d-HPLC (racemic)

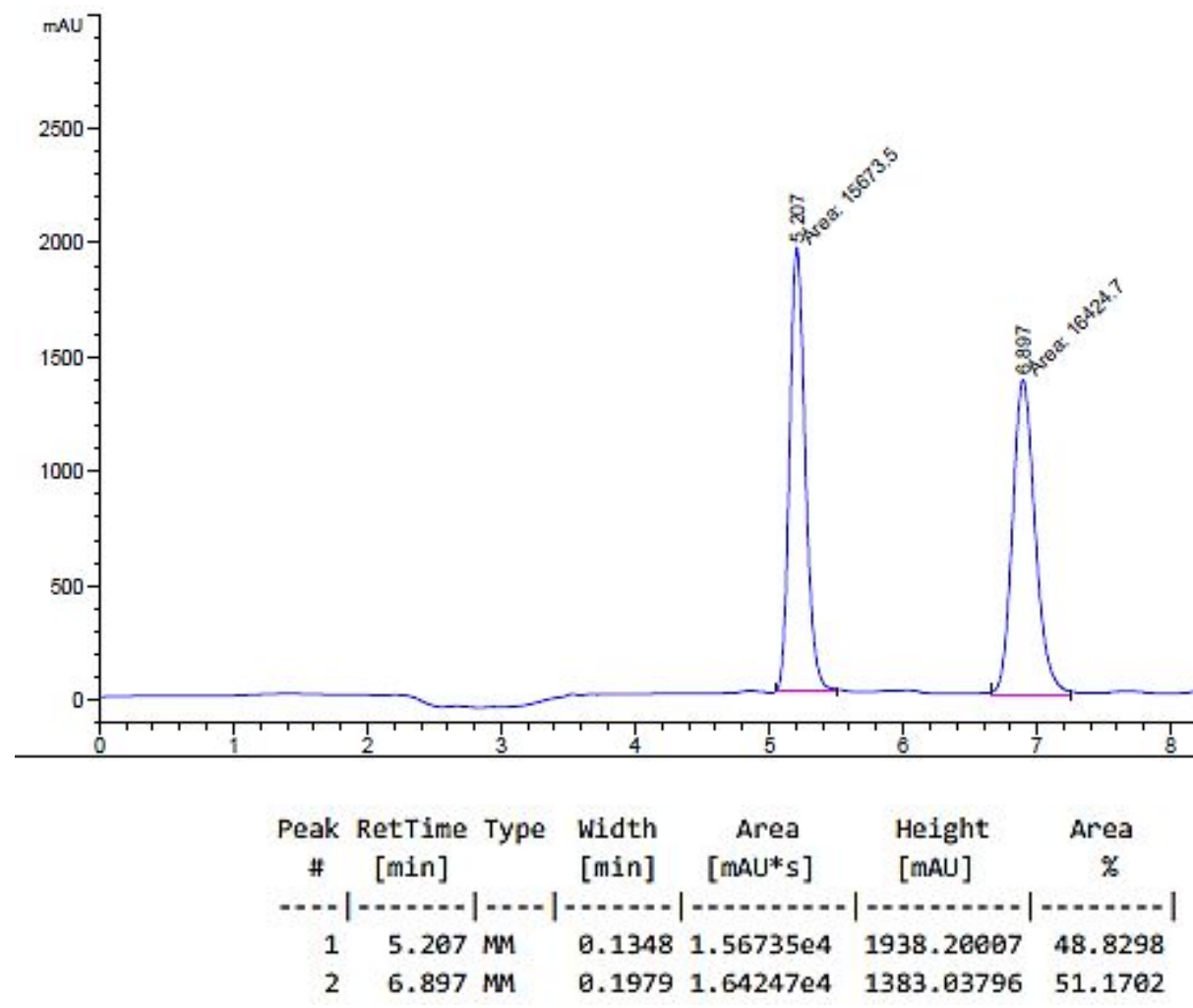

14d-HPLC (90\%) 


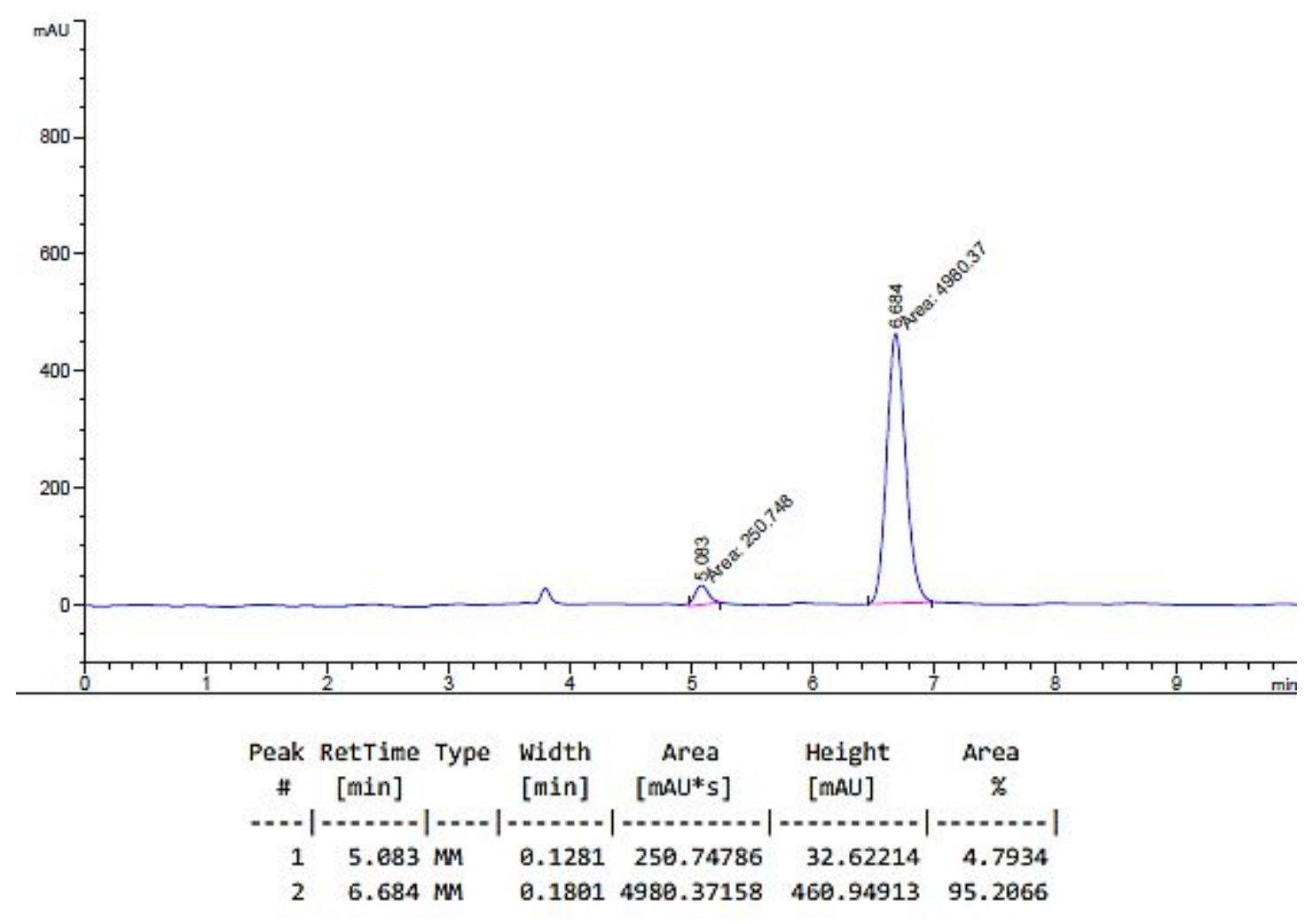

(S,E)-4-(4-fluorophenyl)-6-methyl-1-phenylhept-4-en-3-ol (15d) - ${ }^{1} \mathrm{H}$ NMR

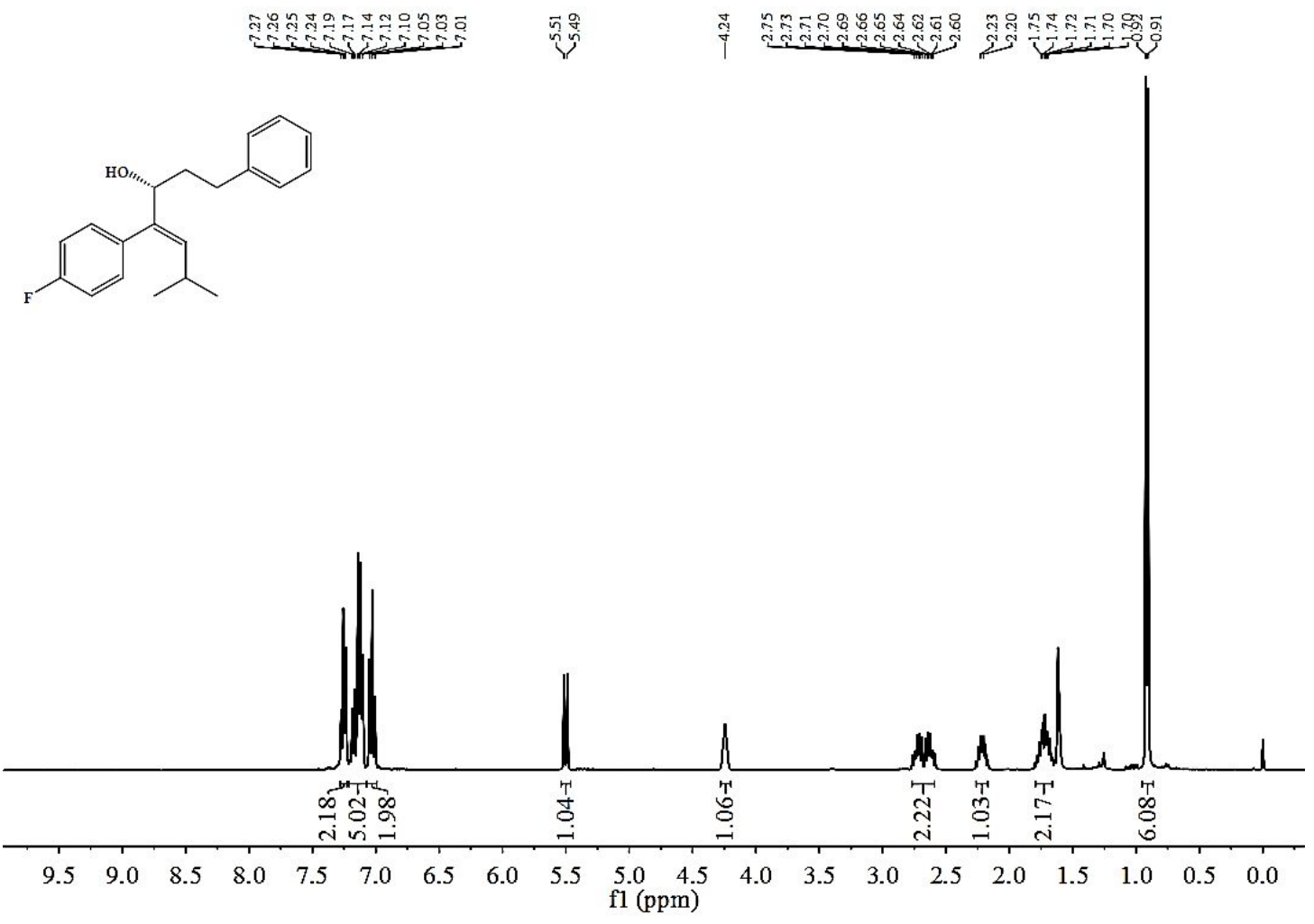

(S,E)-4-(4-fluorophenyl)-6-methyl-1-phenylhept-4-en-3-ol (15d) - ${ }^{13} \mathrm{C}$ NMR 


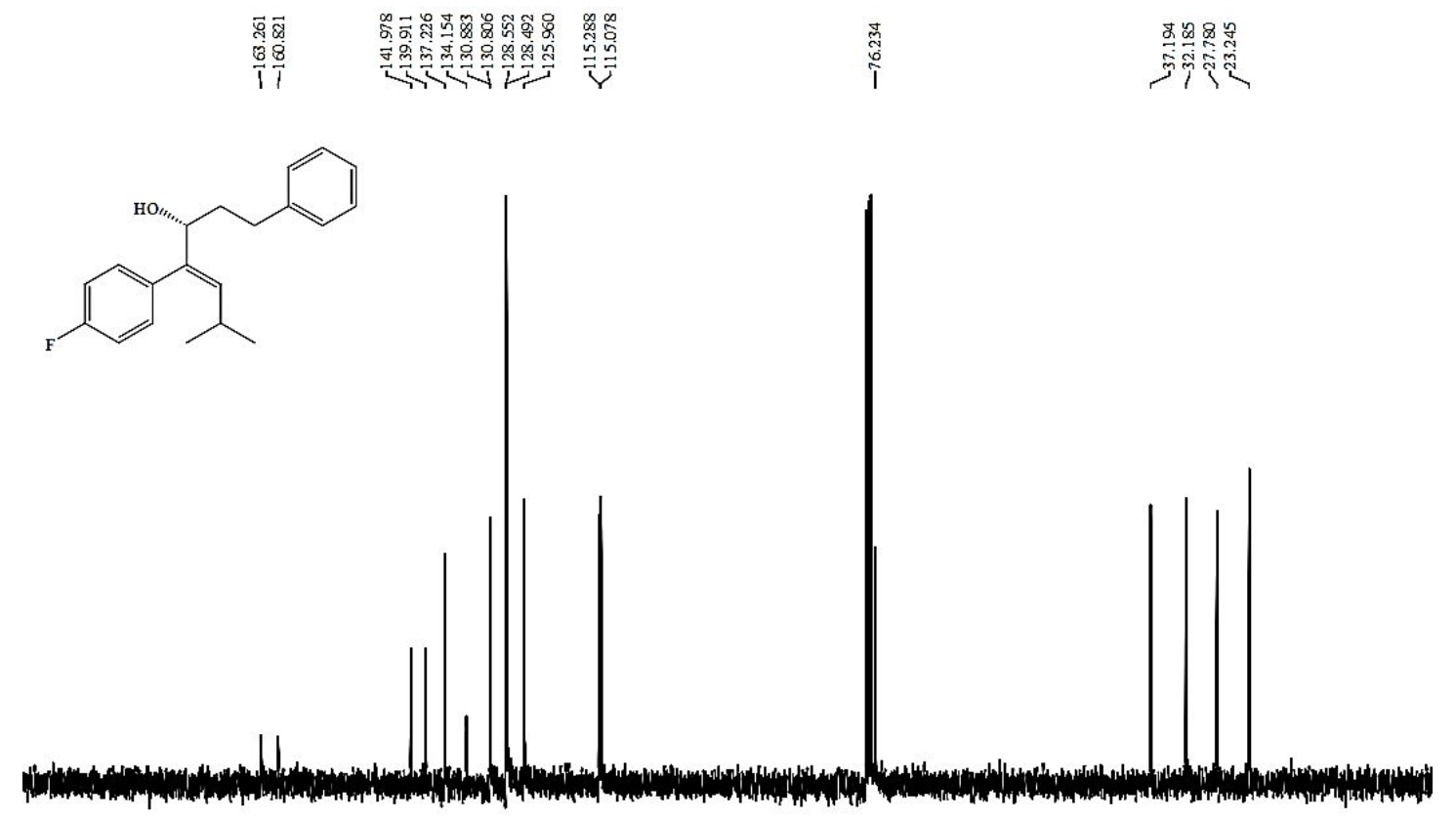

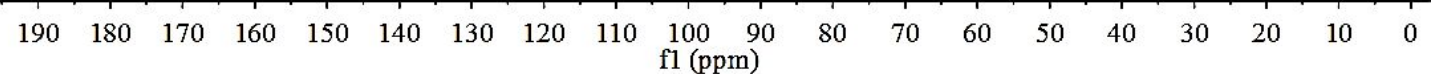

(S,E)-4-(4-fluorophenyl)-6-methyl-1-phenylhept-4-en-3-ol (15d) $-{ }^{19}$ F NMR

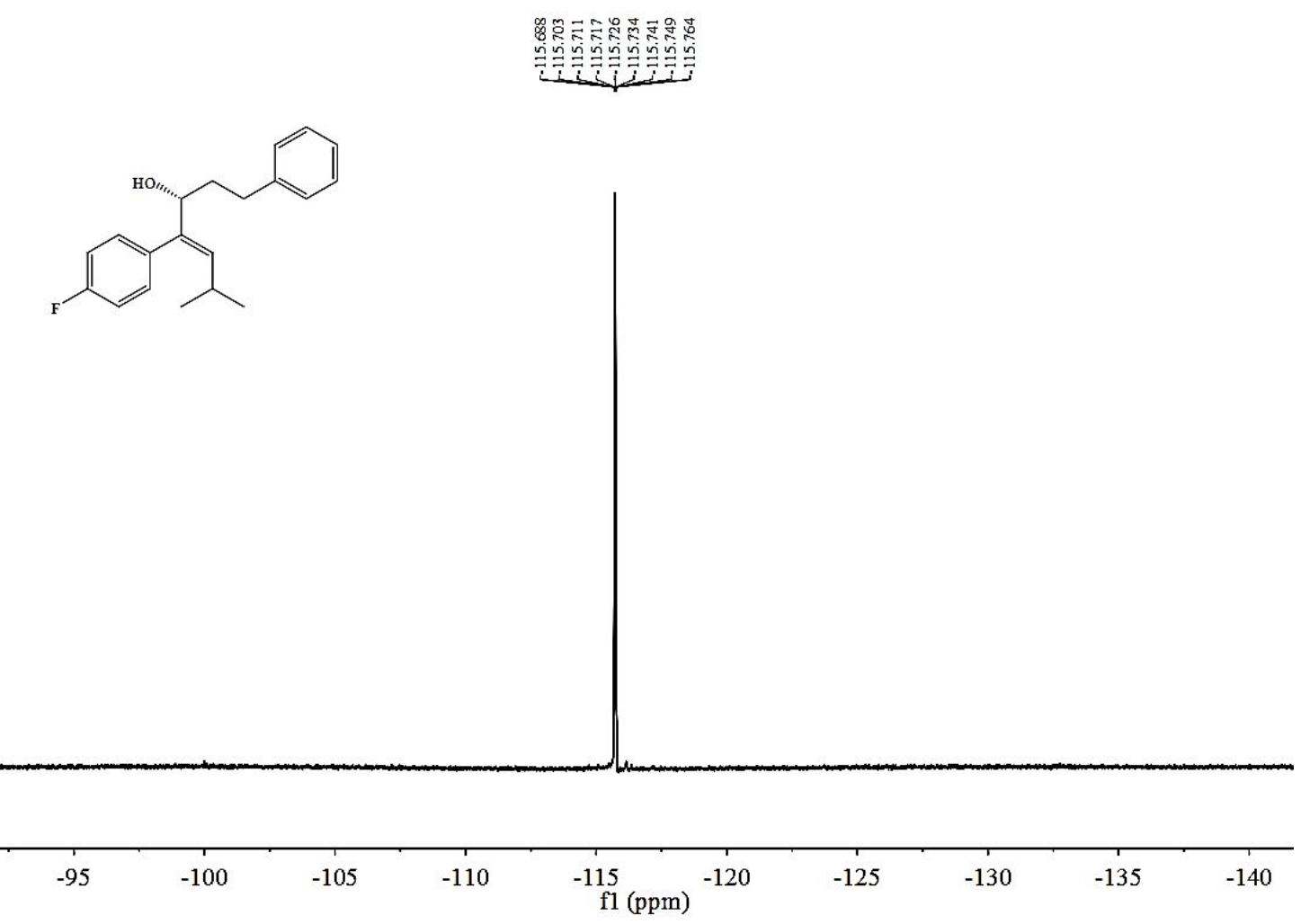

15d-HPLC (racemic) 


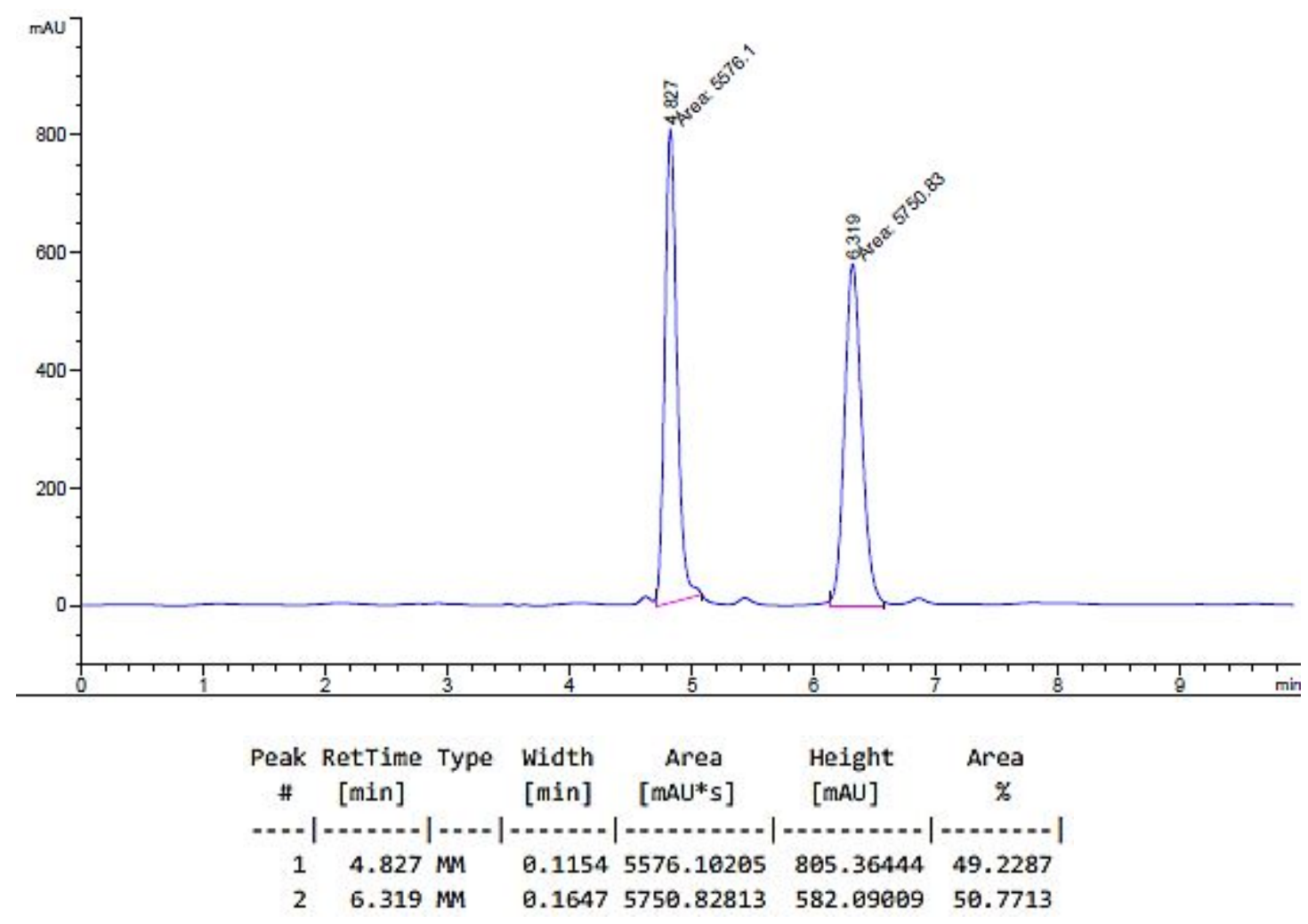

15d-HPLC (96\%)

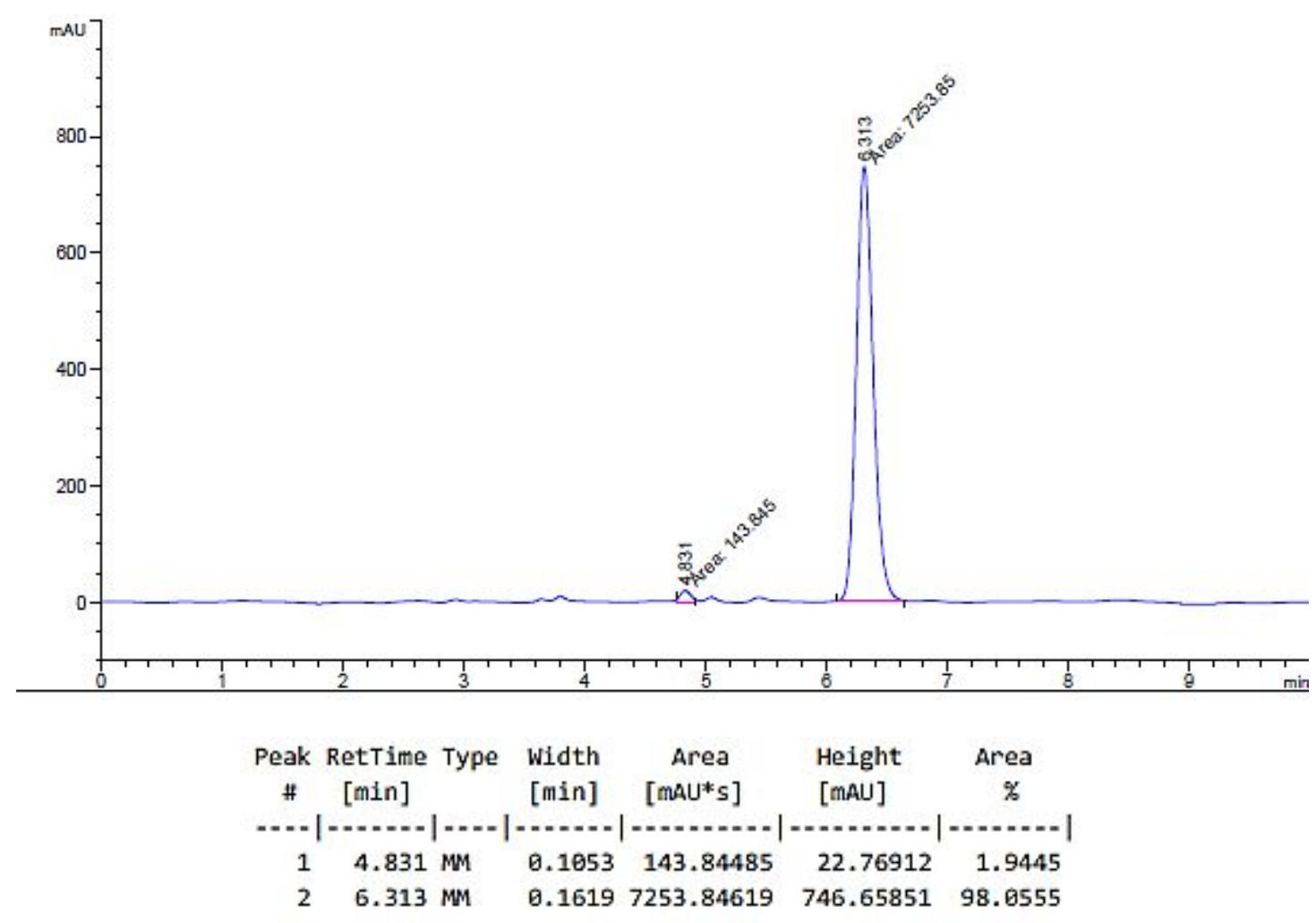

(S,Z)-4-methyl-1-phenyl-2-(thiophen-2-yl)pent-2-en-1-ol (16d) - ${ }^{1} \mathrm{H}$ NMR 

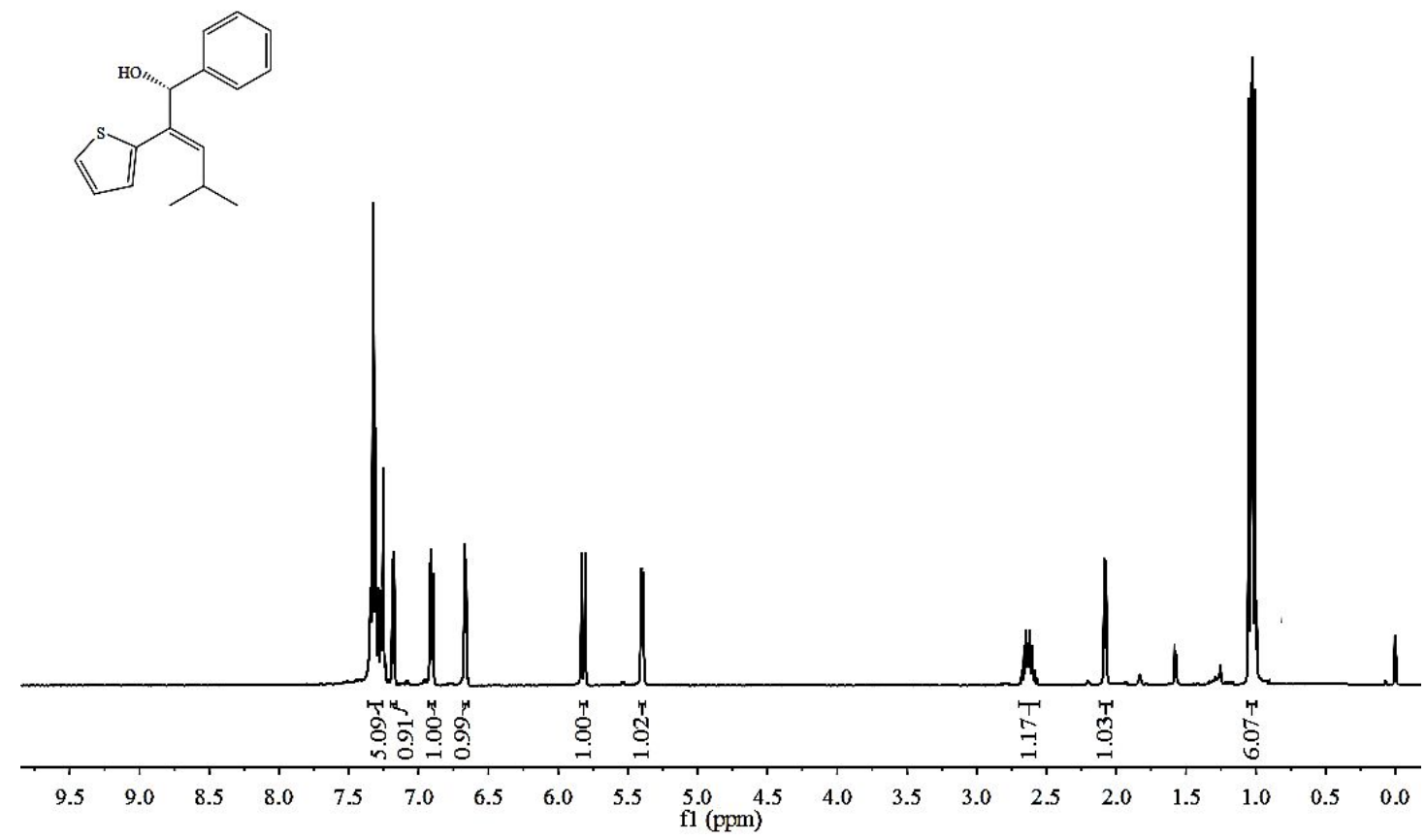

(S,Z)-4-methyl-1-phenyl-2-(thiophen-2-yl)pent-2-en-1-ol (16d) - ${ }^{13} \mathrm{C}$ NMR

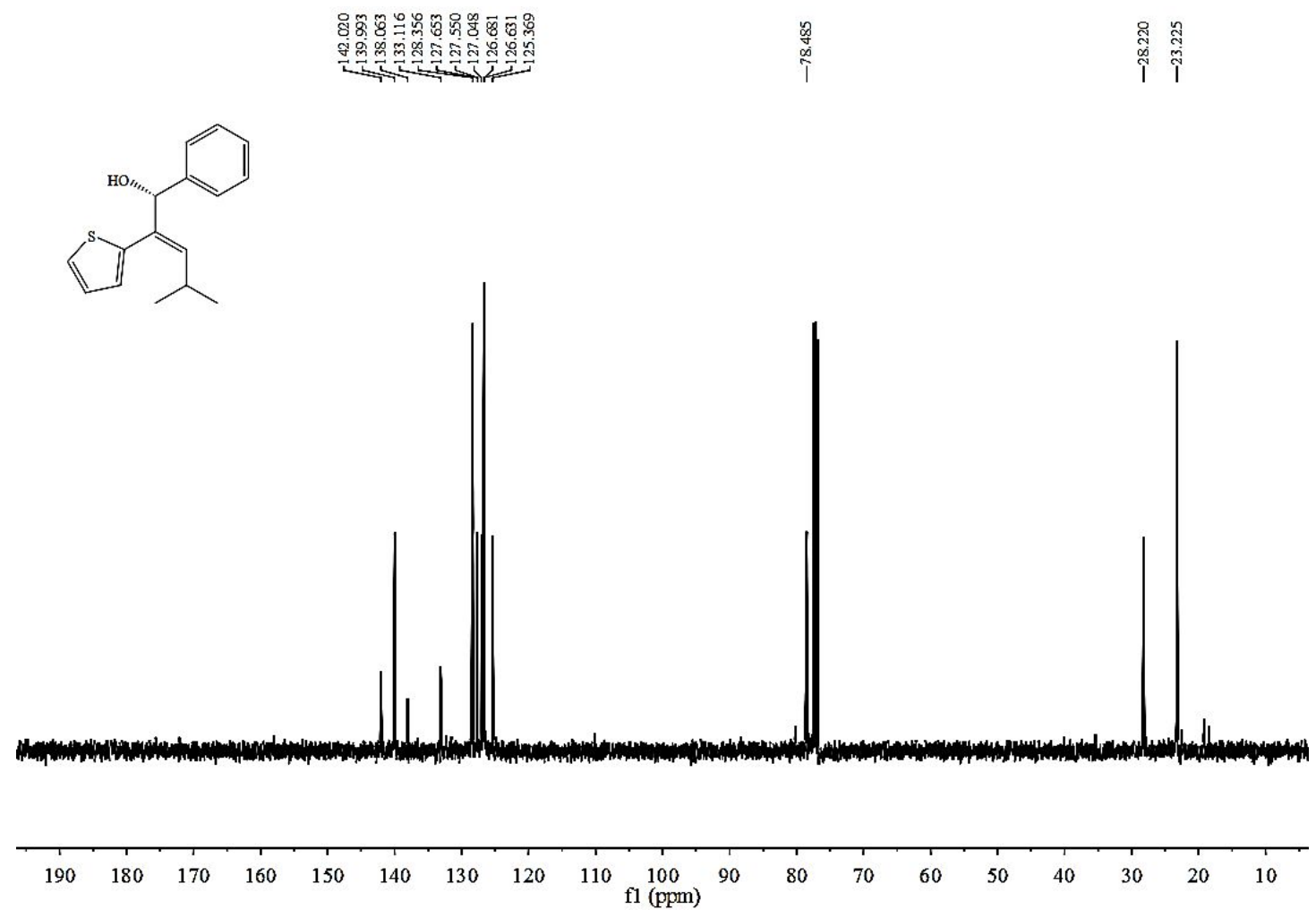

16d-HPLC (racemic) 


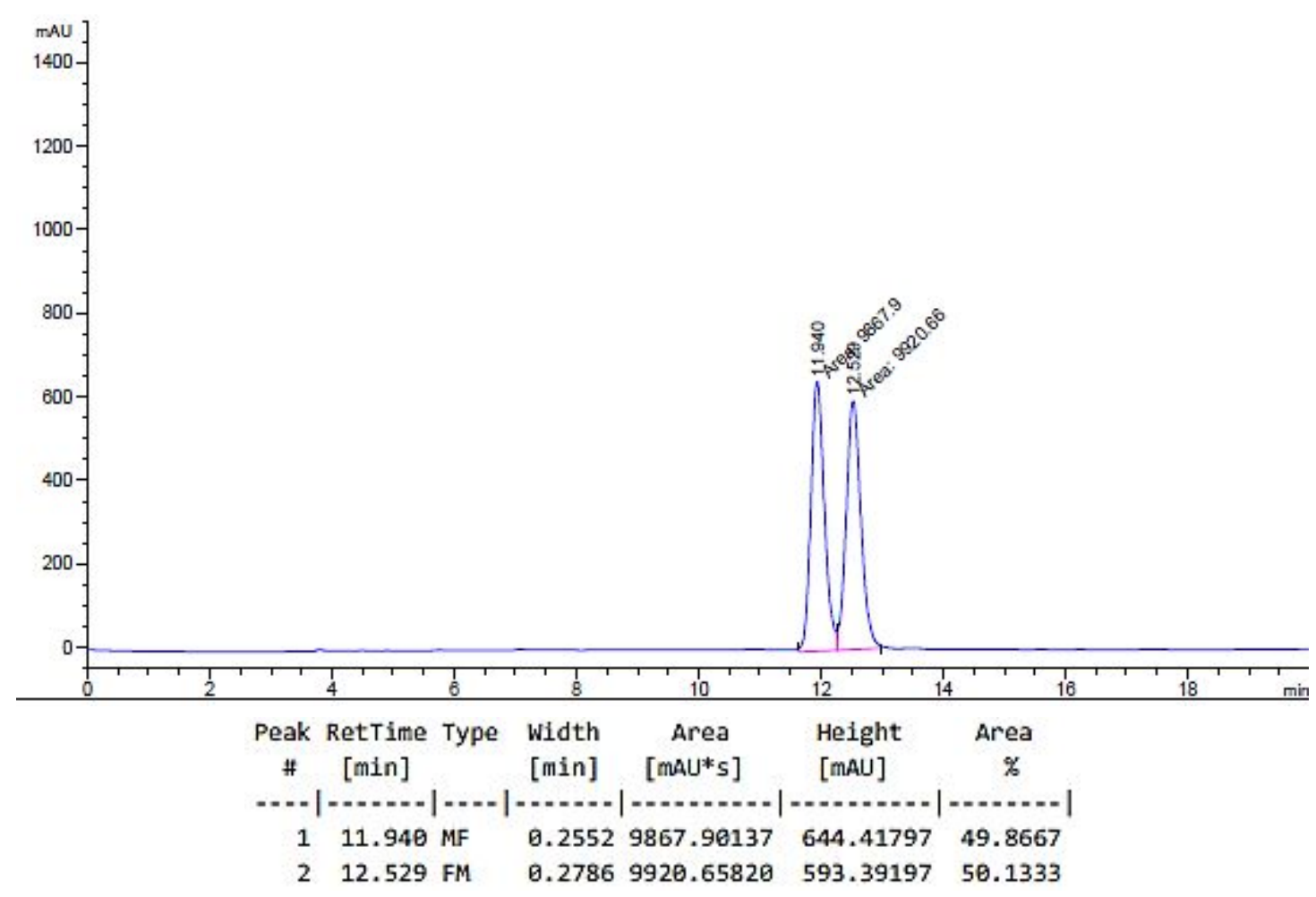

16d-HPLC (90\%)

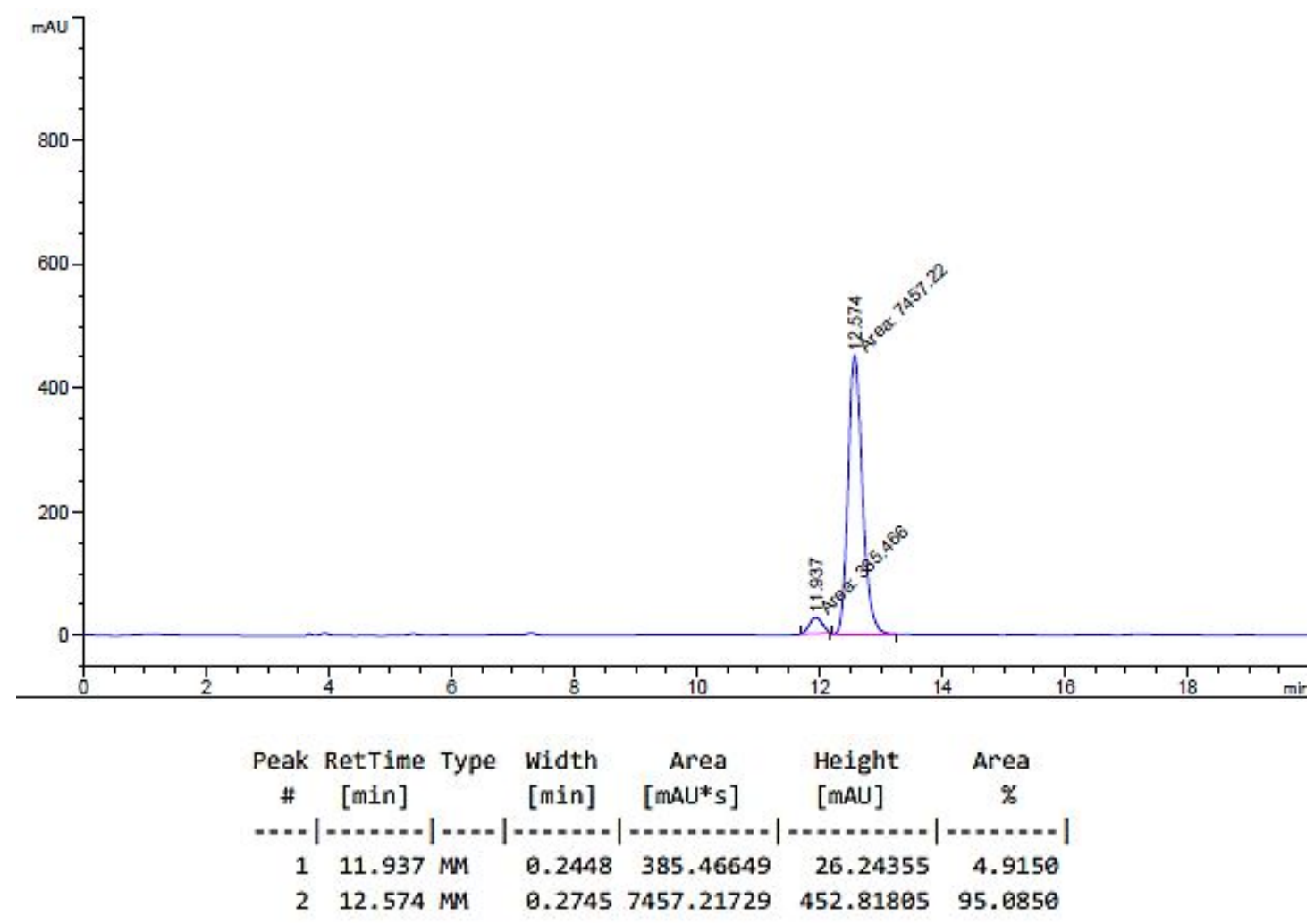

(S,Z)-6-methyl-1-phenyl-4-(trimethylsilyl)hept-4-en-3-ol (17d) - ${ }^{1} \mathrm{H}$ NMR 


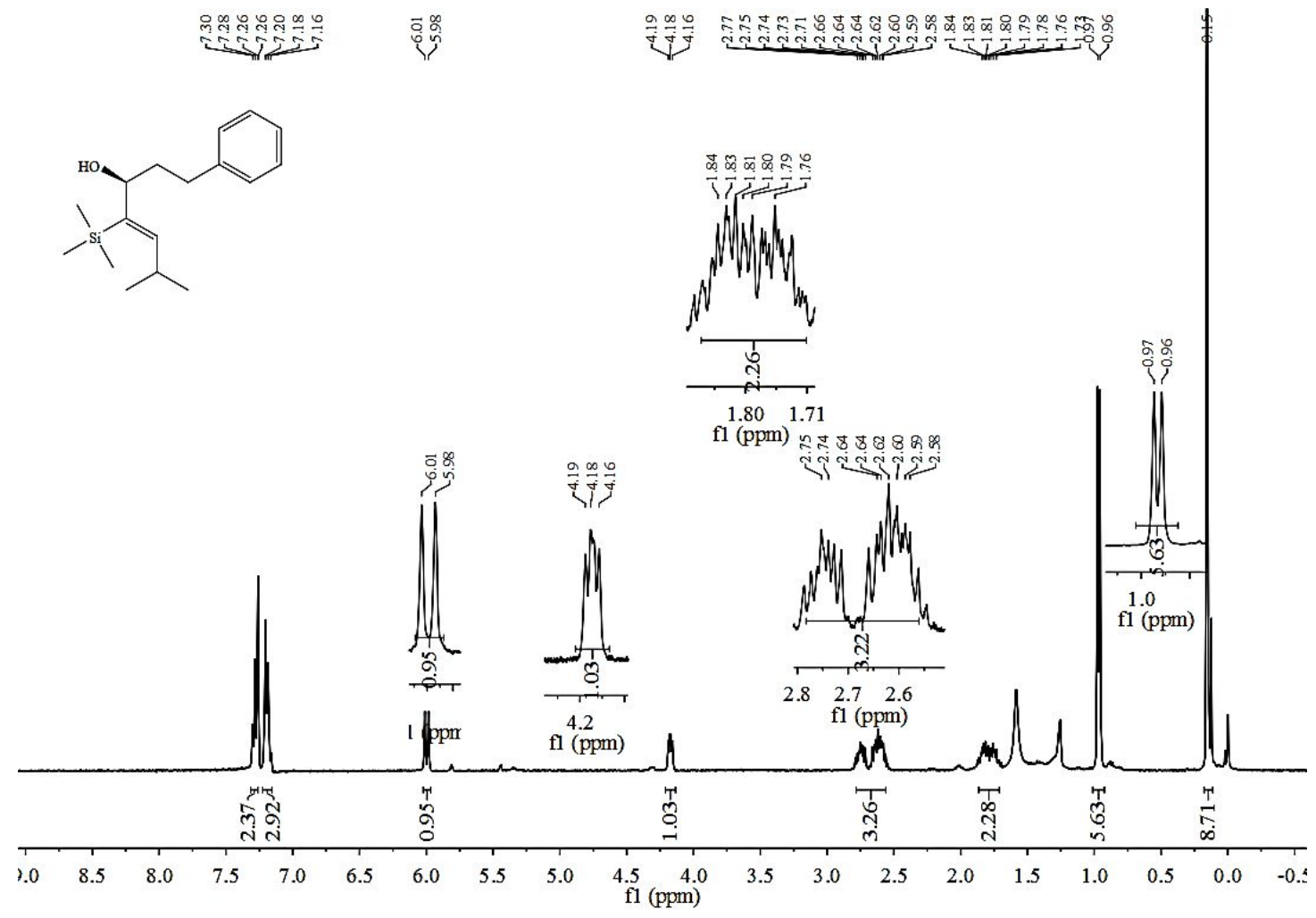

(S,Z)-6-methyl-1-phenyl-4-(trimethylsilyl)hept-4-en-3-ol (17d) - ${ }^{13} \mathrm{C}$ NMR
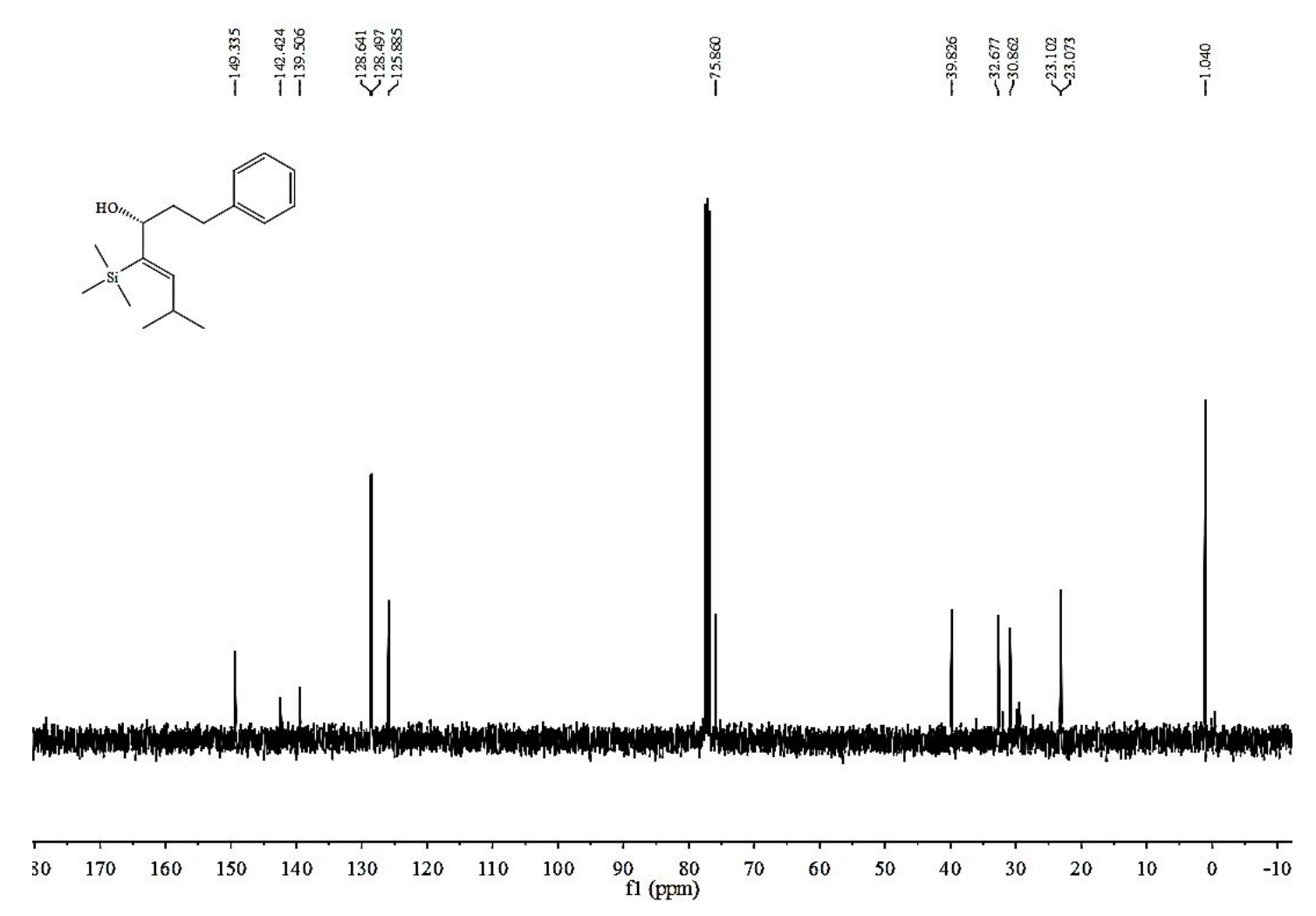

17d-HPLC (racemic) 


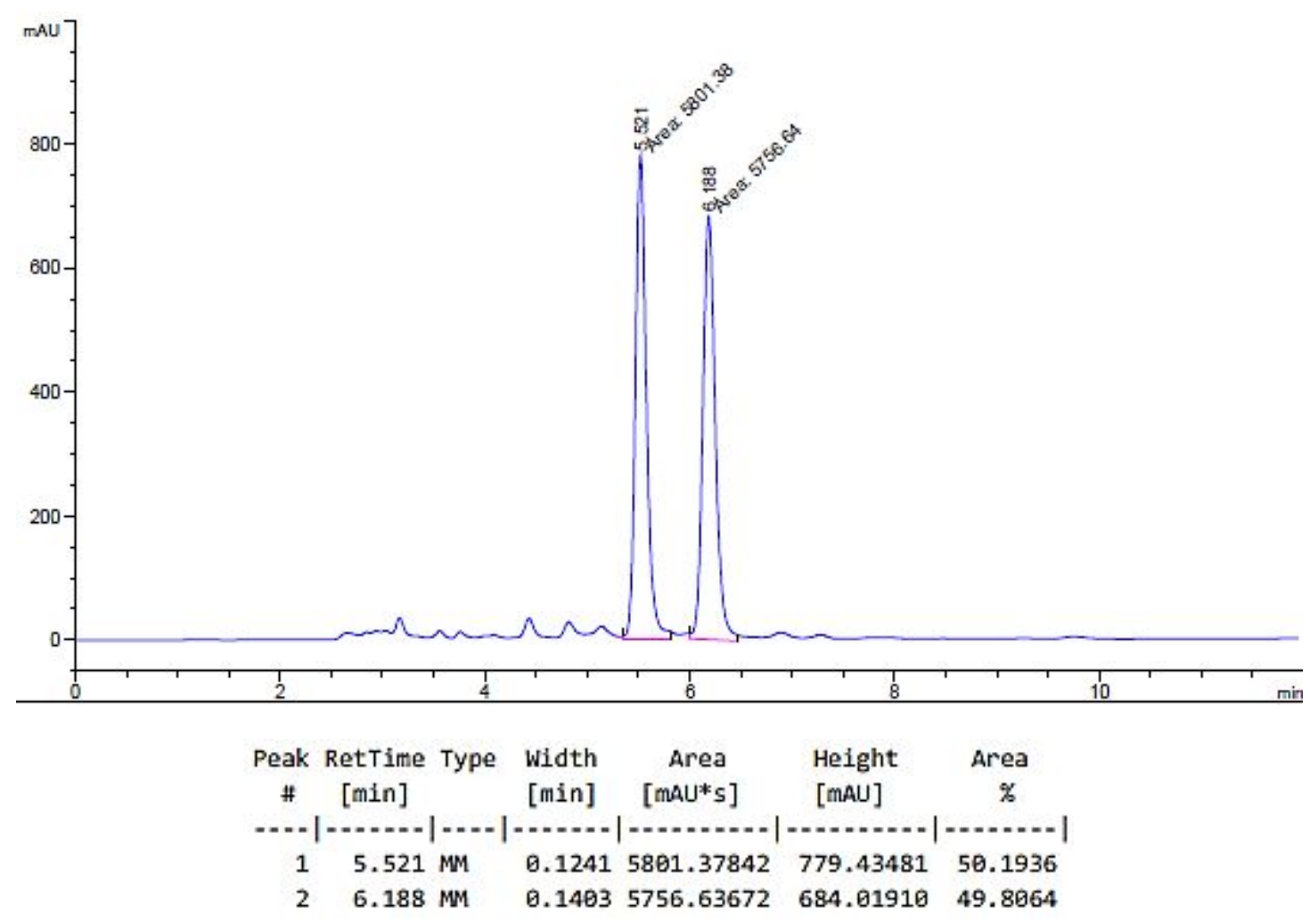

17d-HPLC (94\%)

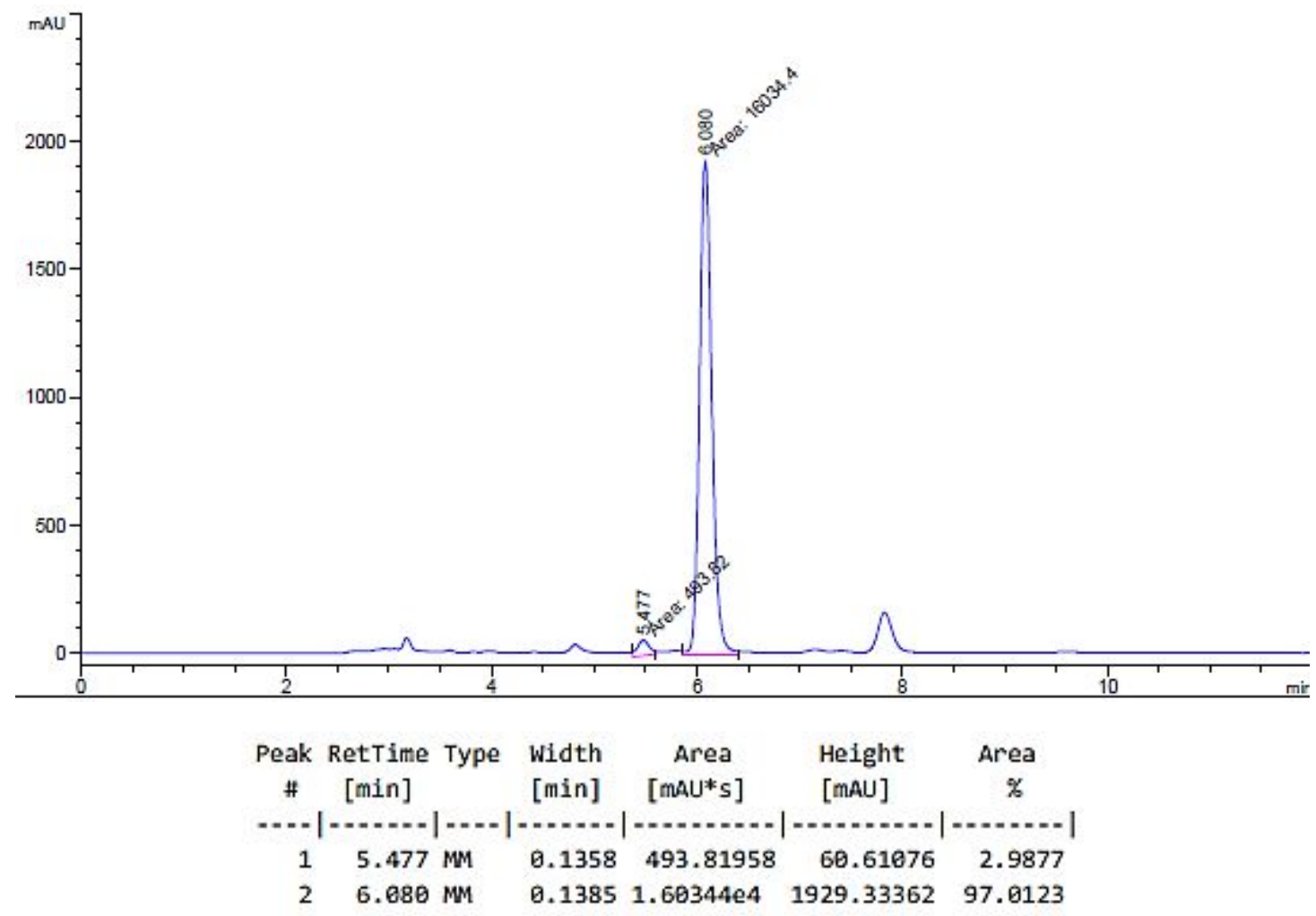

(S,E)-1-cyclohexyl-2,5-diphenylpent-1-en-3-ol (18d) - ${ }^{1} \mathrm{H}$ NMR 


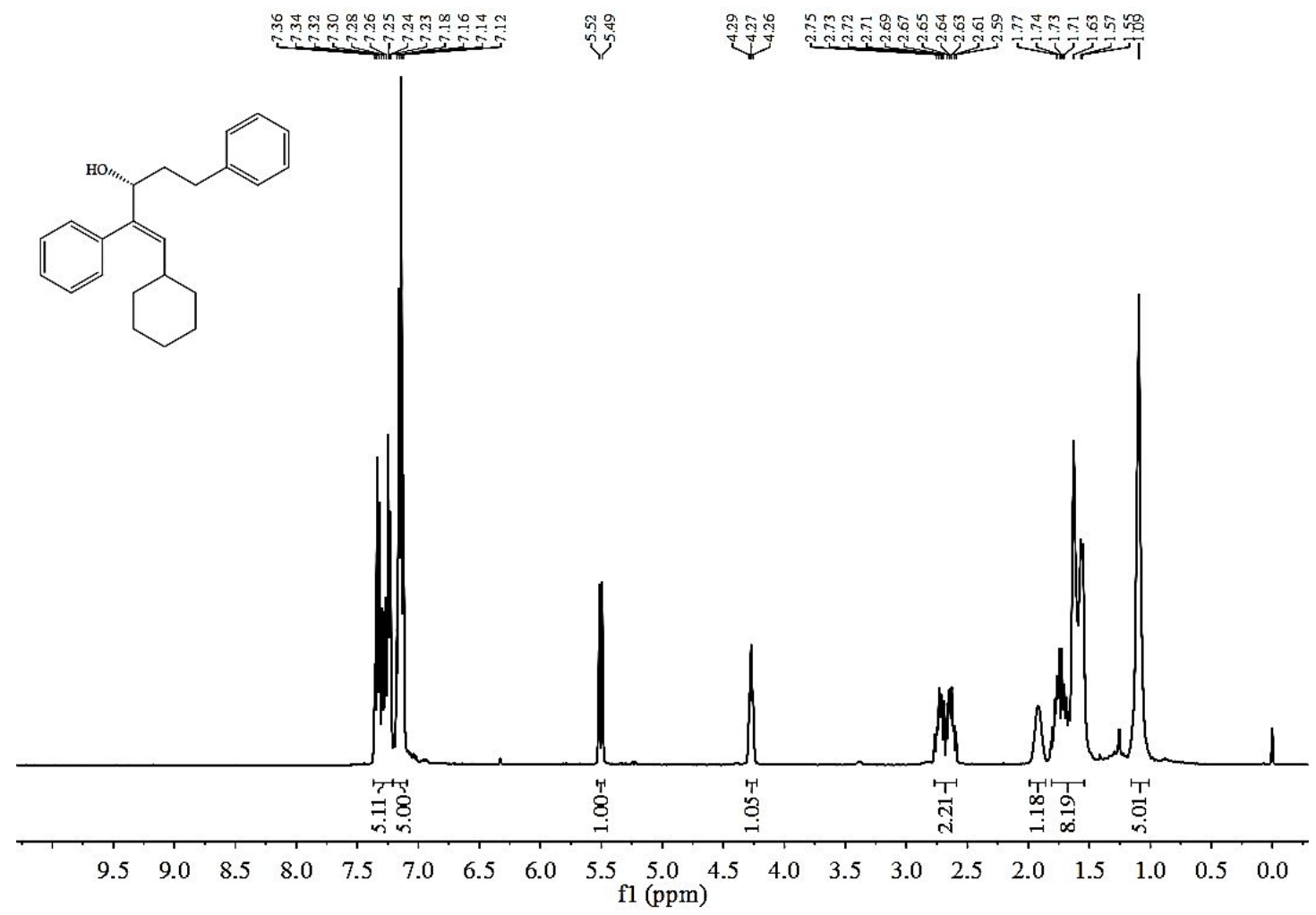

(S,E)-1-cyclohexyl-2,5-diphenylpent-1-en-3-ol (18d) - ${ }^{13}$ C NMR

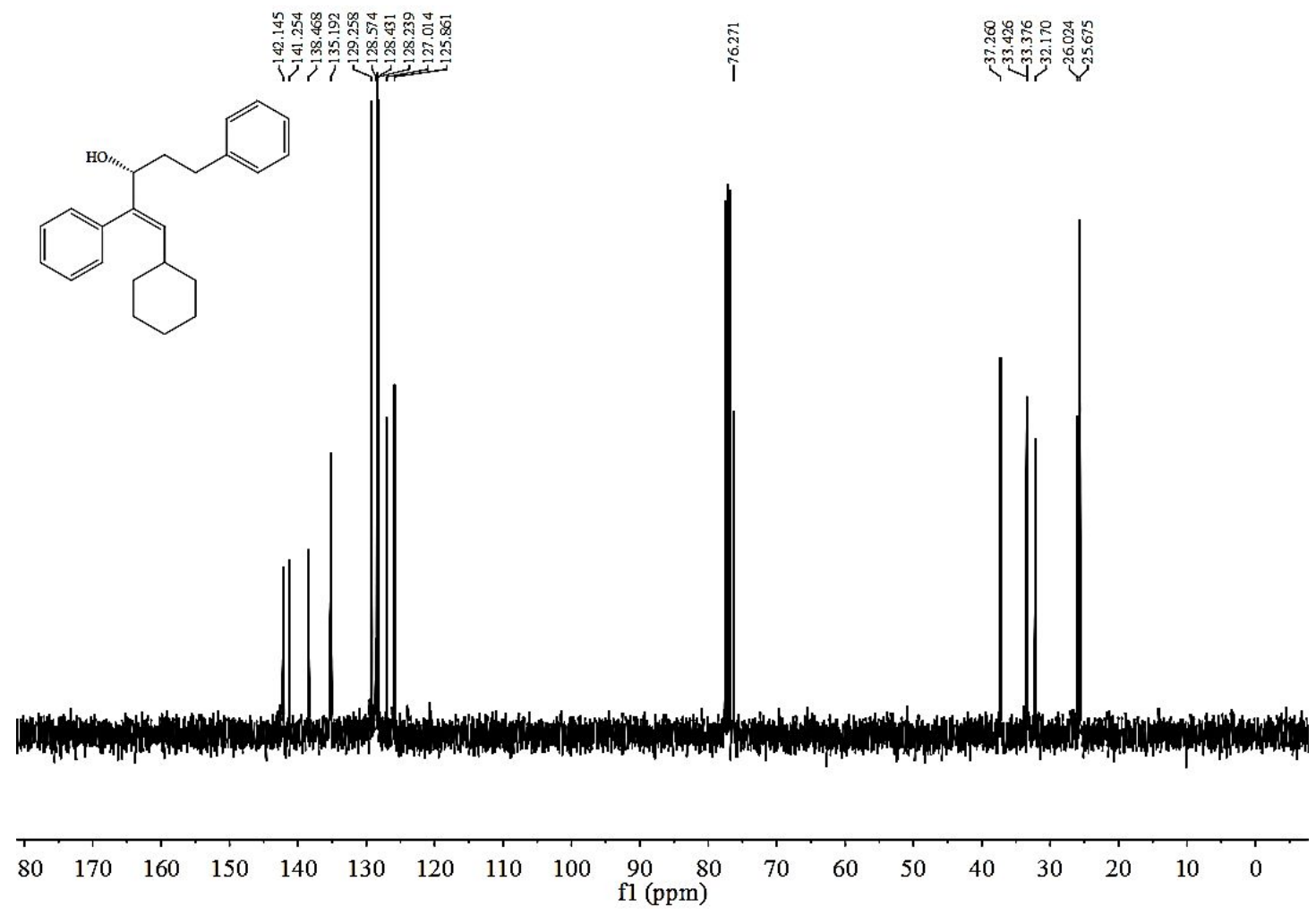

18d-HPLC (racemic) 


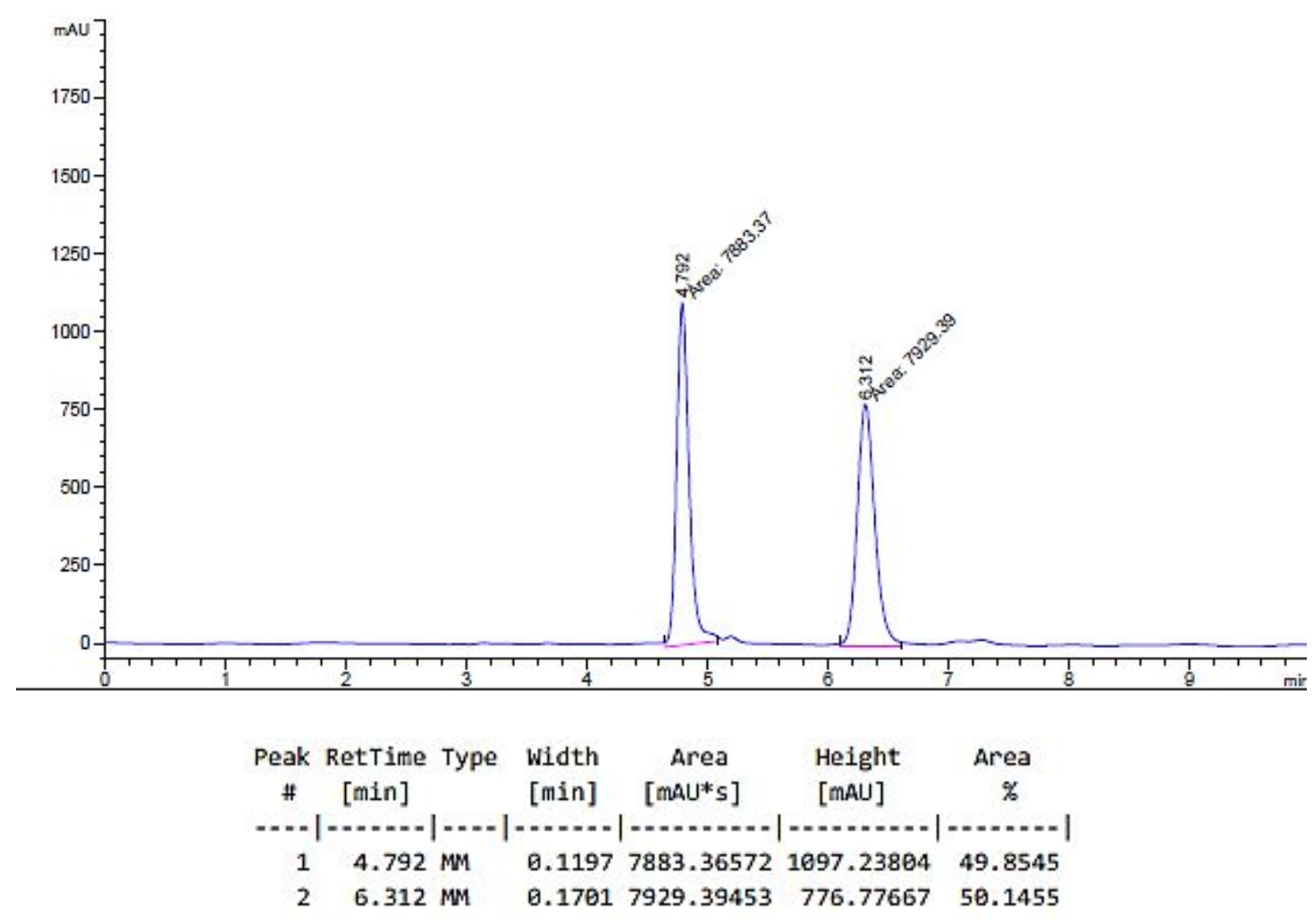

18d-HPLC (95\%)

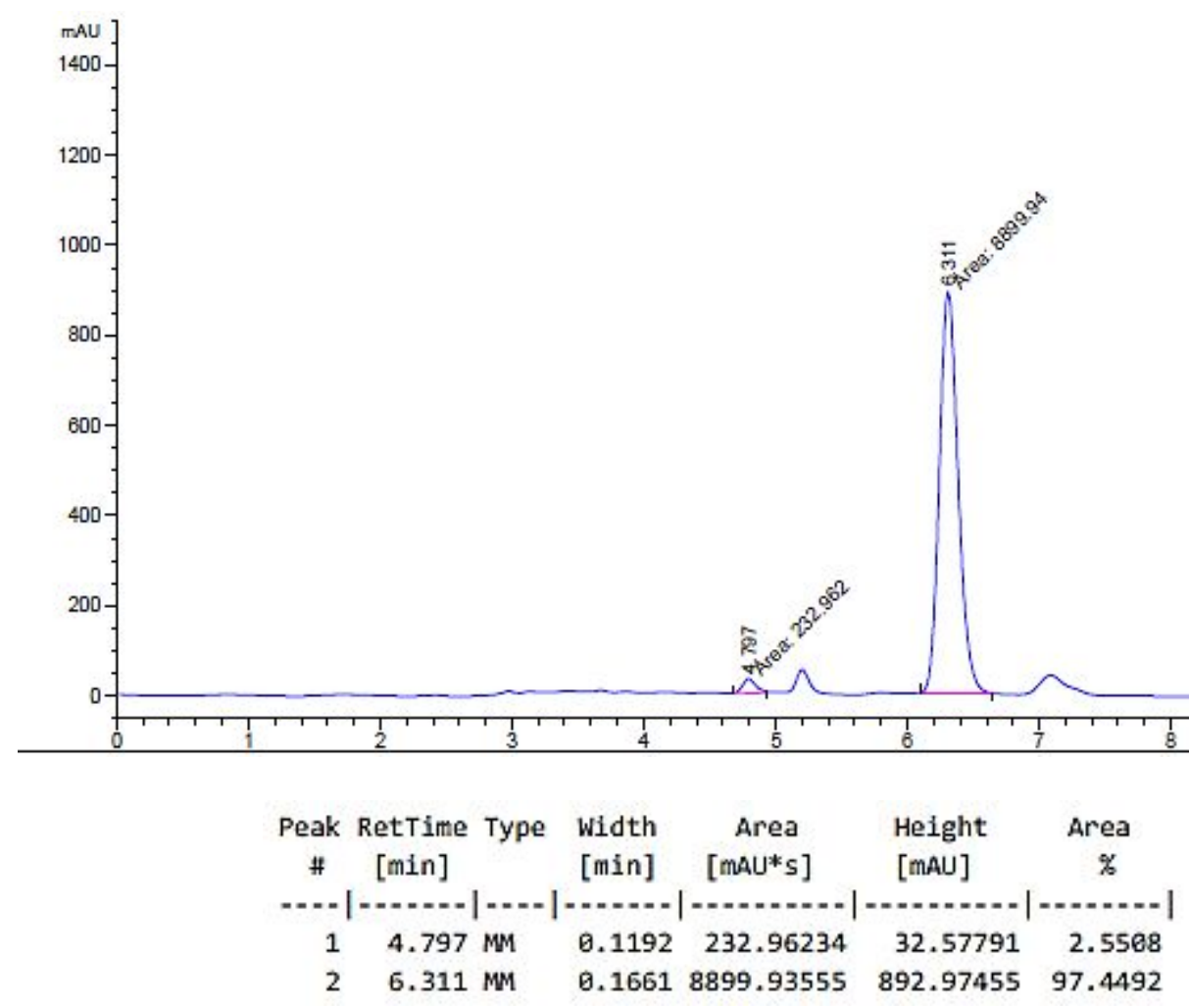

(S,E)-3-cyclohexyl-1,2-diphenylprop-2-en-1-ol (19d) - ${ }^{1} \mathrm{H}$ NMR 


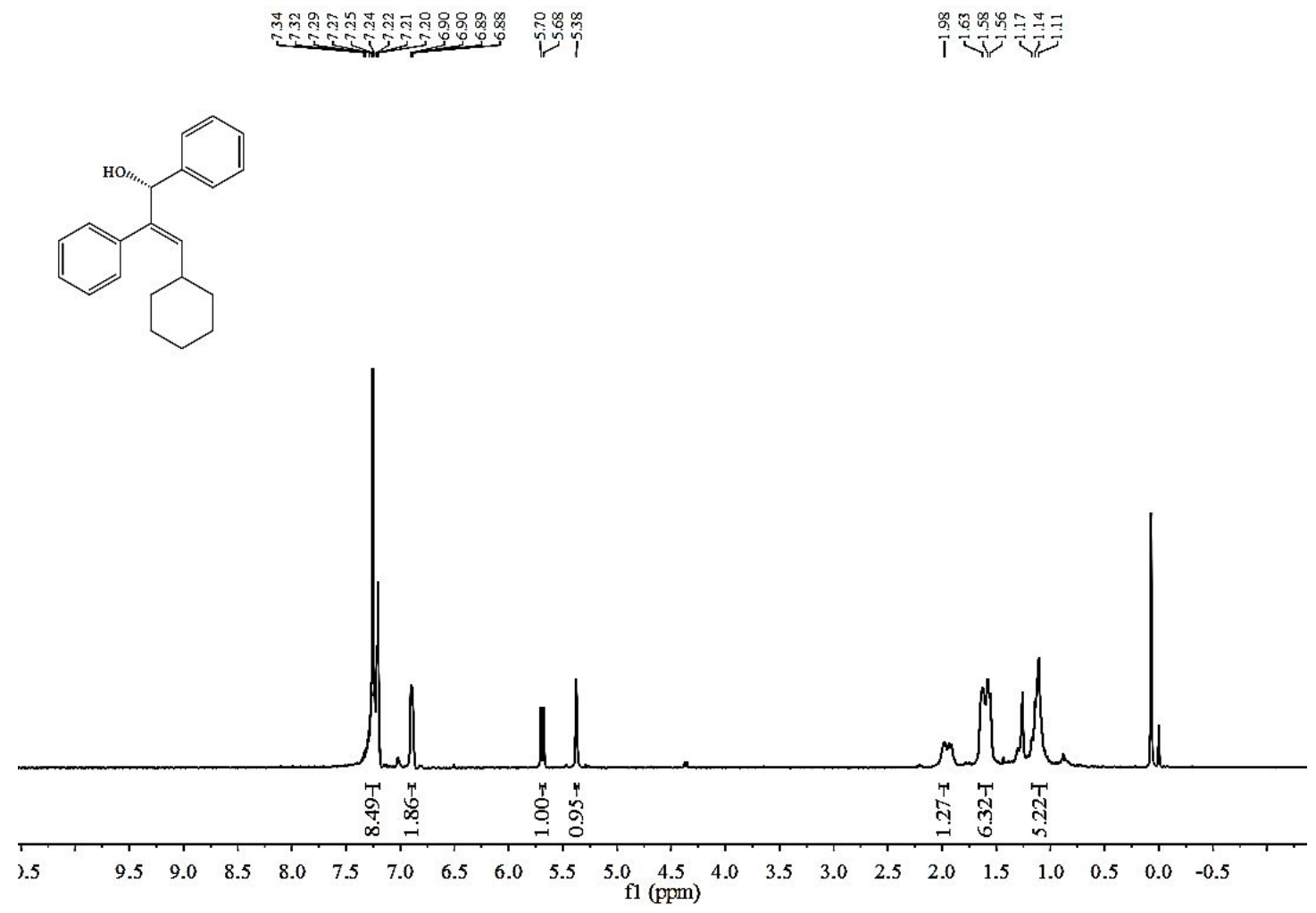

(S,E)-3-cyclohexyl-1,2-diphenylprop-2-en-1-ol (19d) - ${ }^{13}$ C NMR

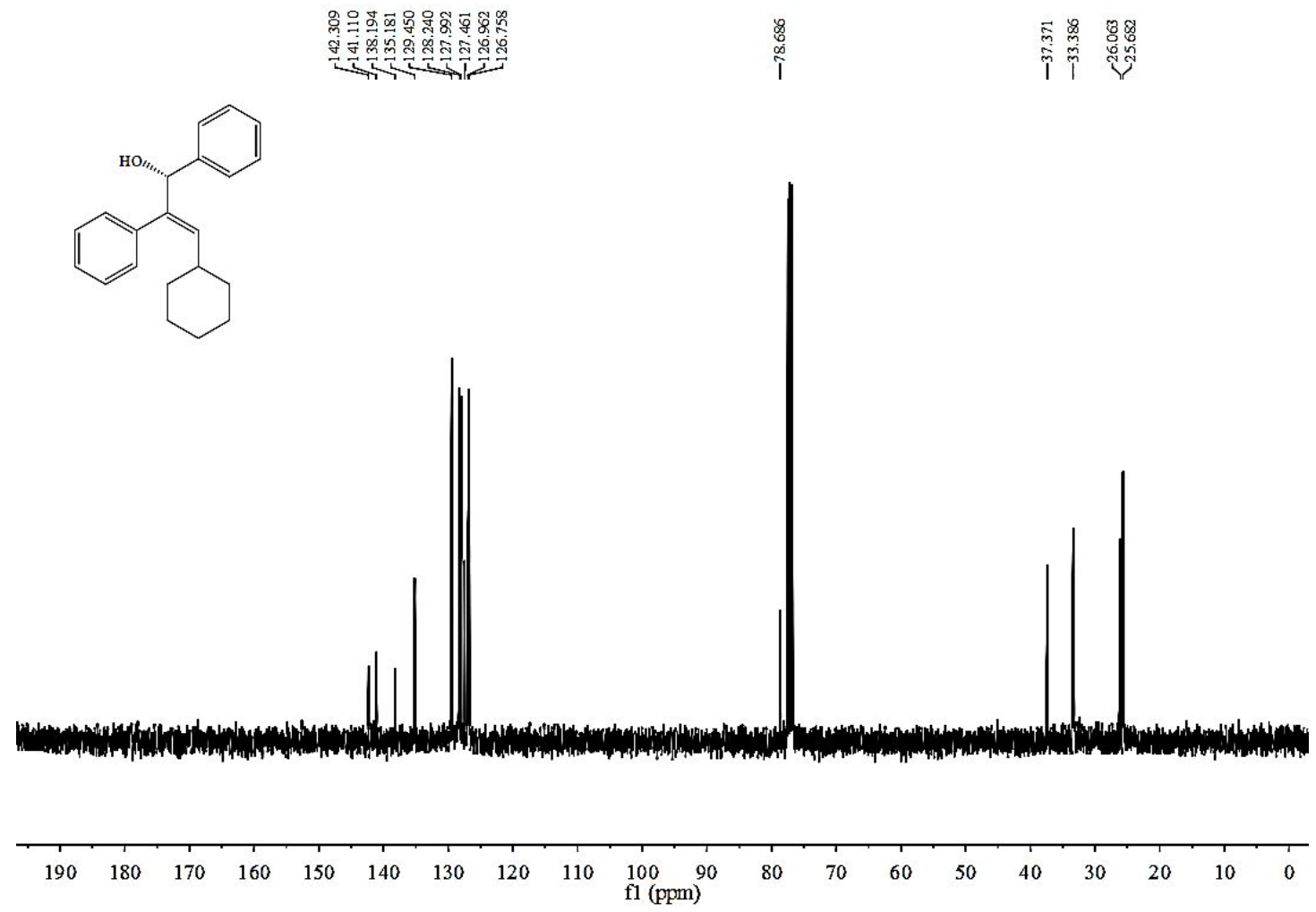

19d-HPLC (racemic) 


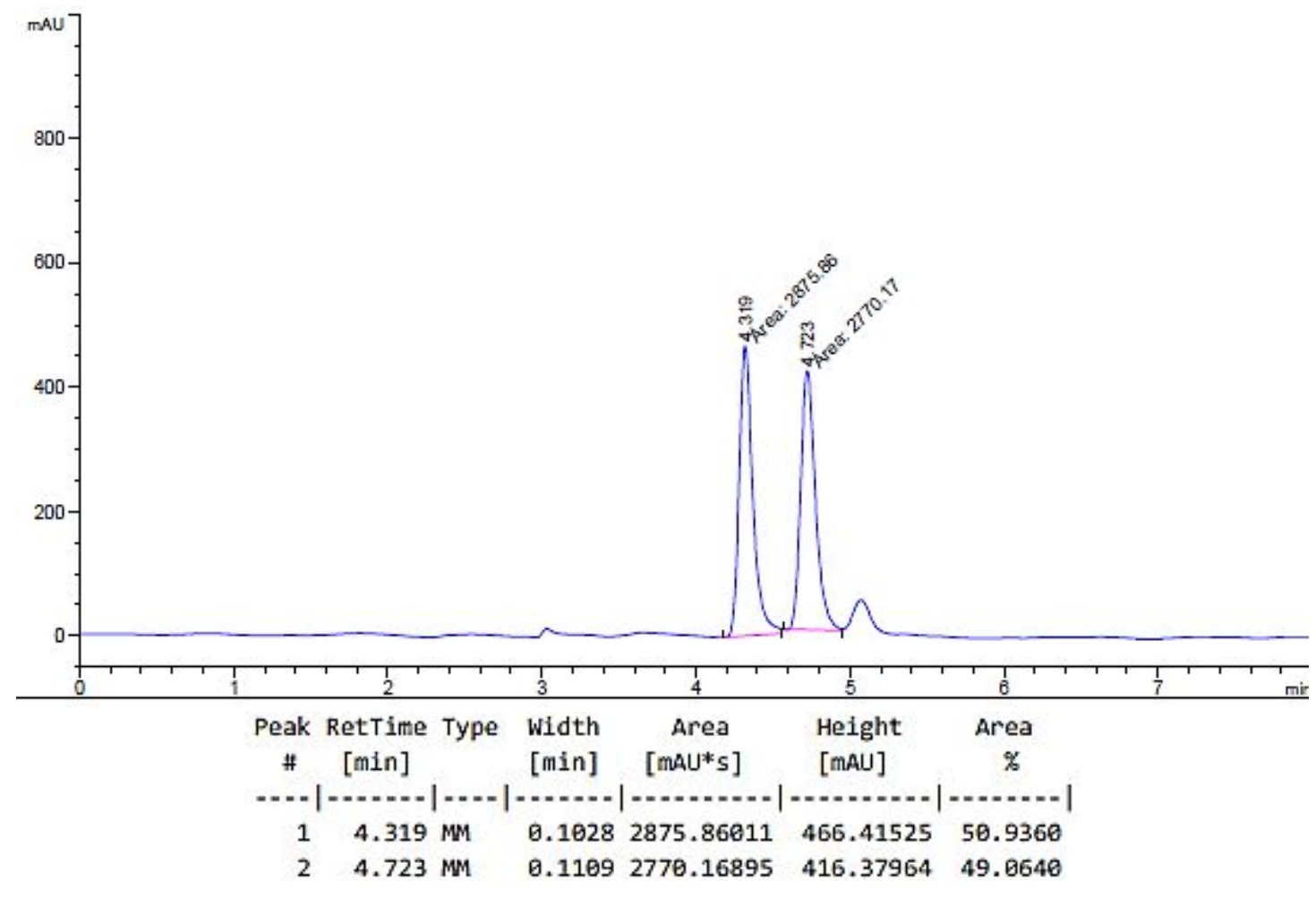

19d-HPLC (96\%)

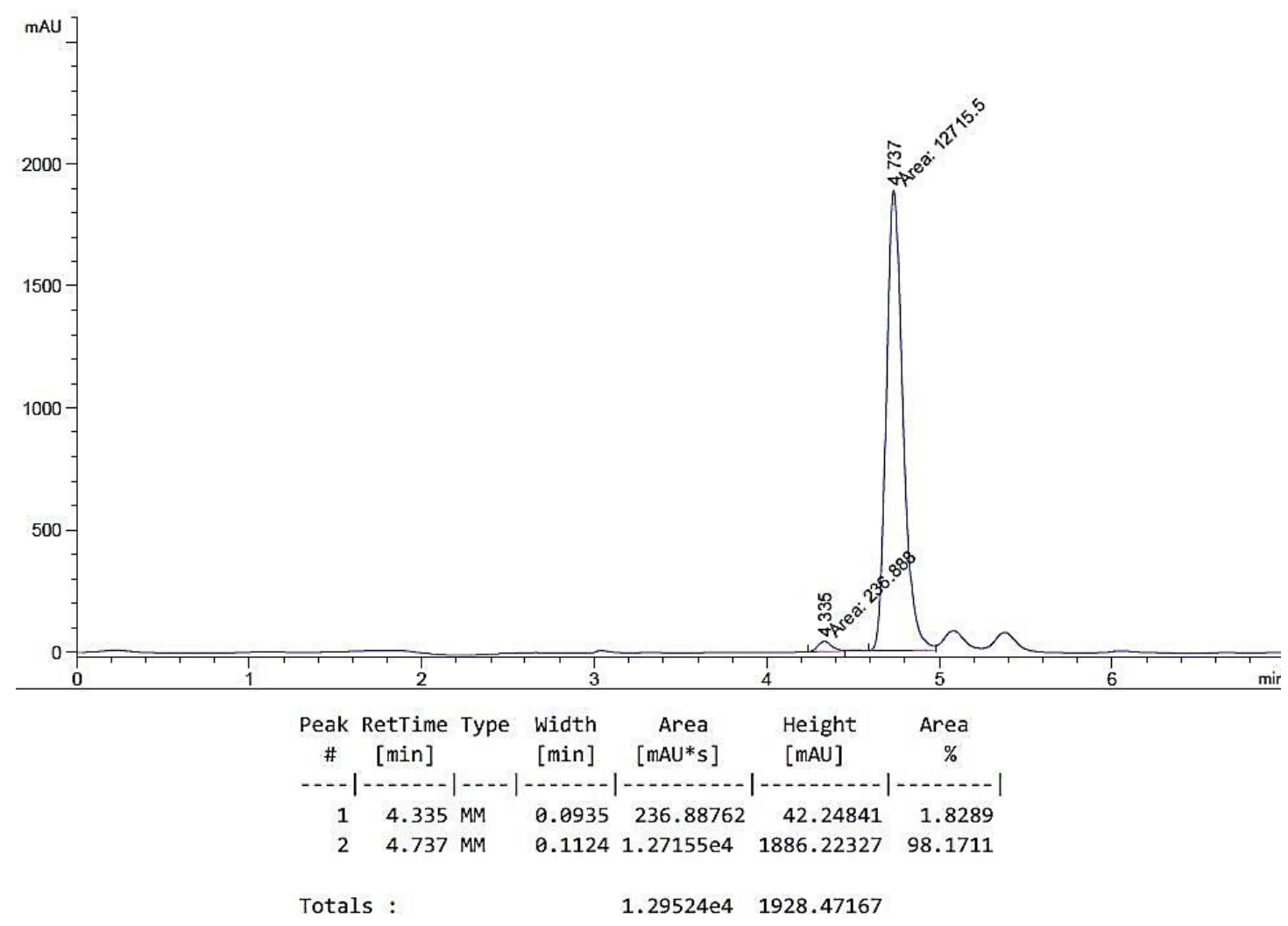

(S,E)-2,5-diphenyl-1-(tetrahydro-2H-pyran-4-yl)pent-1-en-3-ol (20d) - ${ }^{1} \mathrm{H}$ NMR 


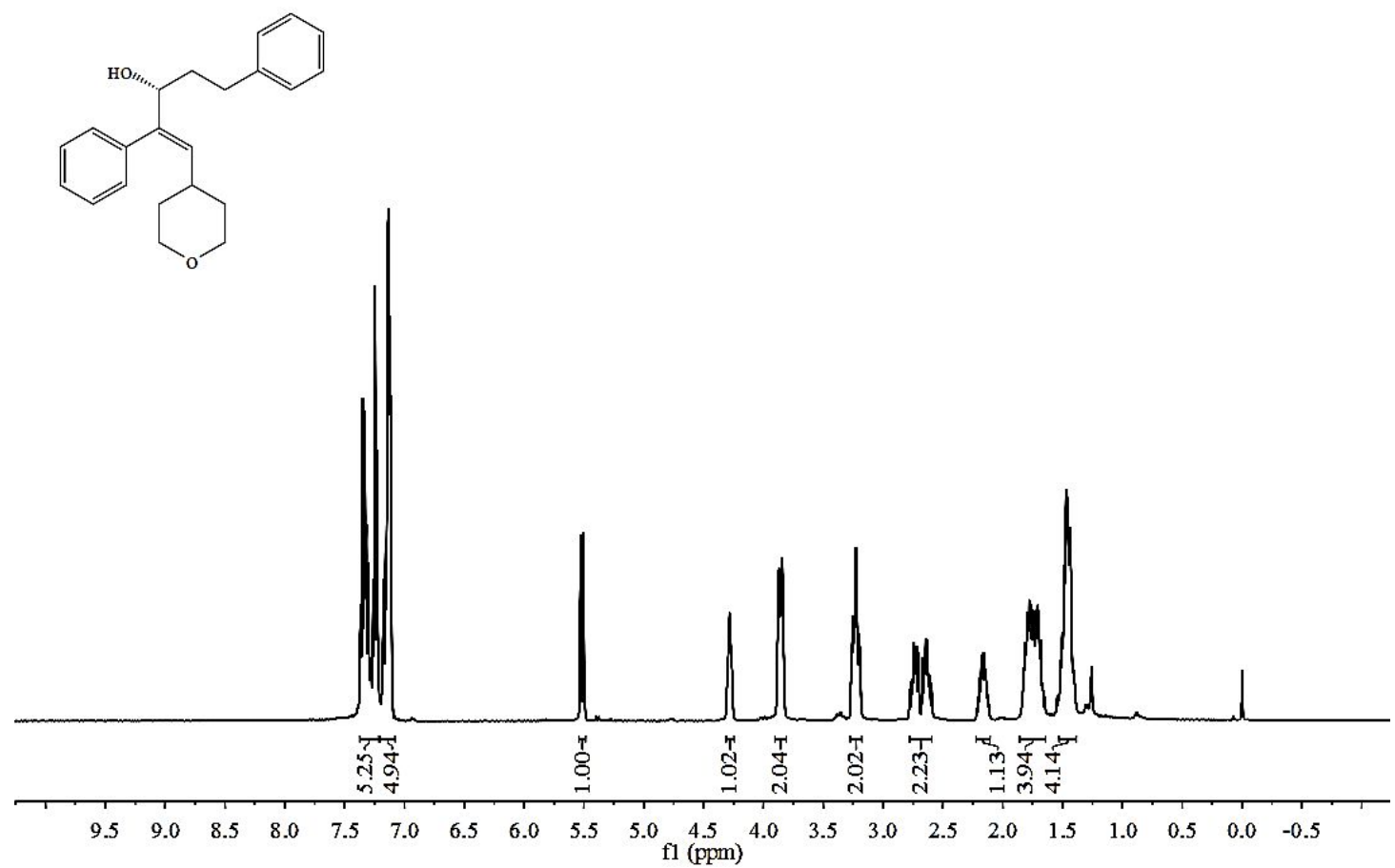

(S,E)-2,5-diphenyl-1-(tetrahydro-2H-pyran-4-yl)pent-1-en-3-ol (20d) $-{ }^{13} \mathrm{C}$ NMR

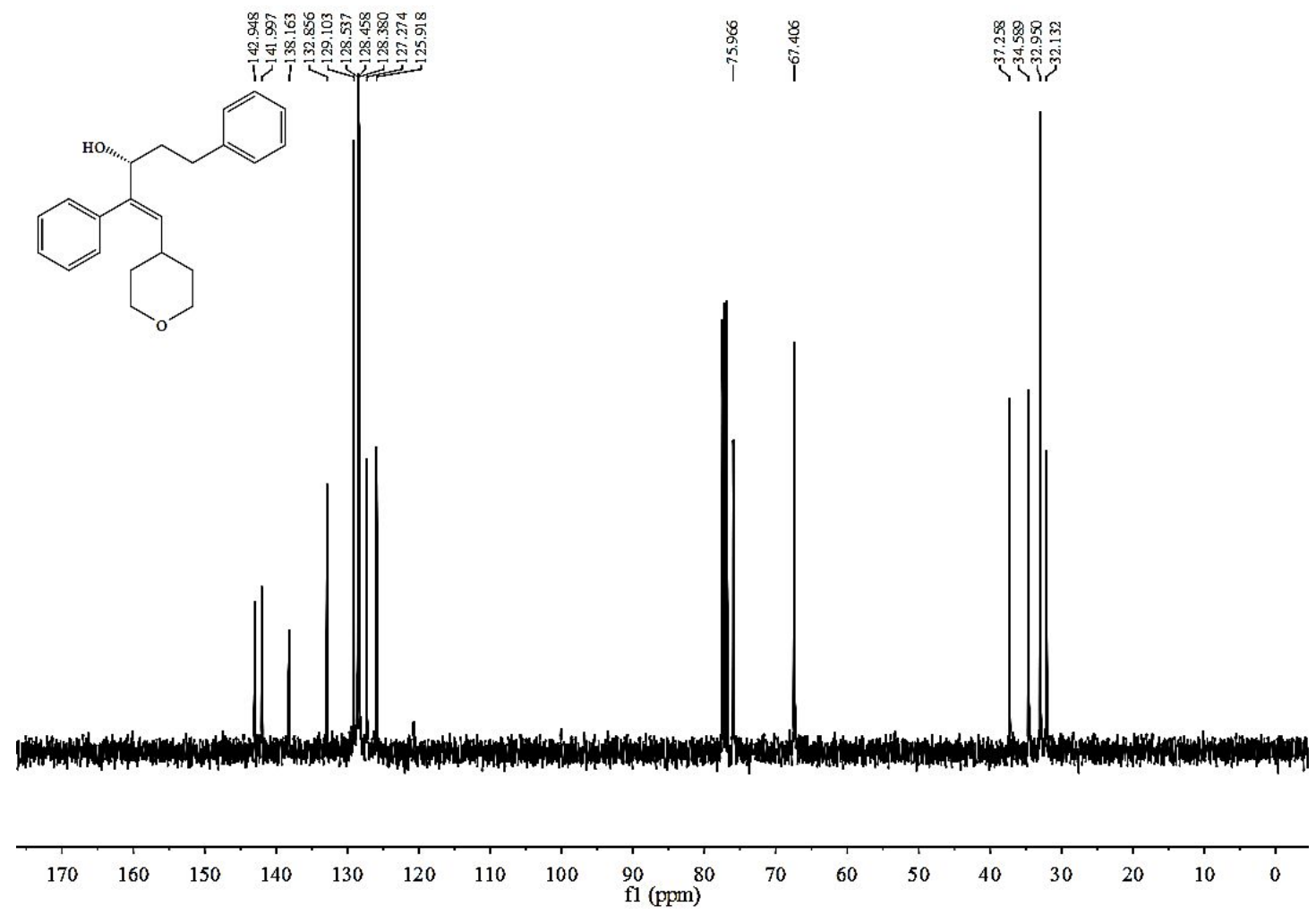

20d-HPLC (racemic) 

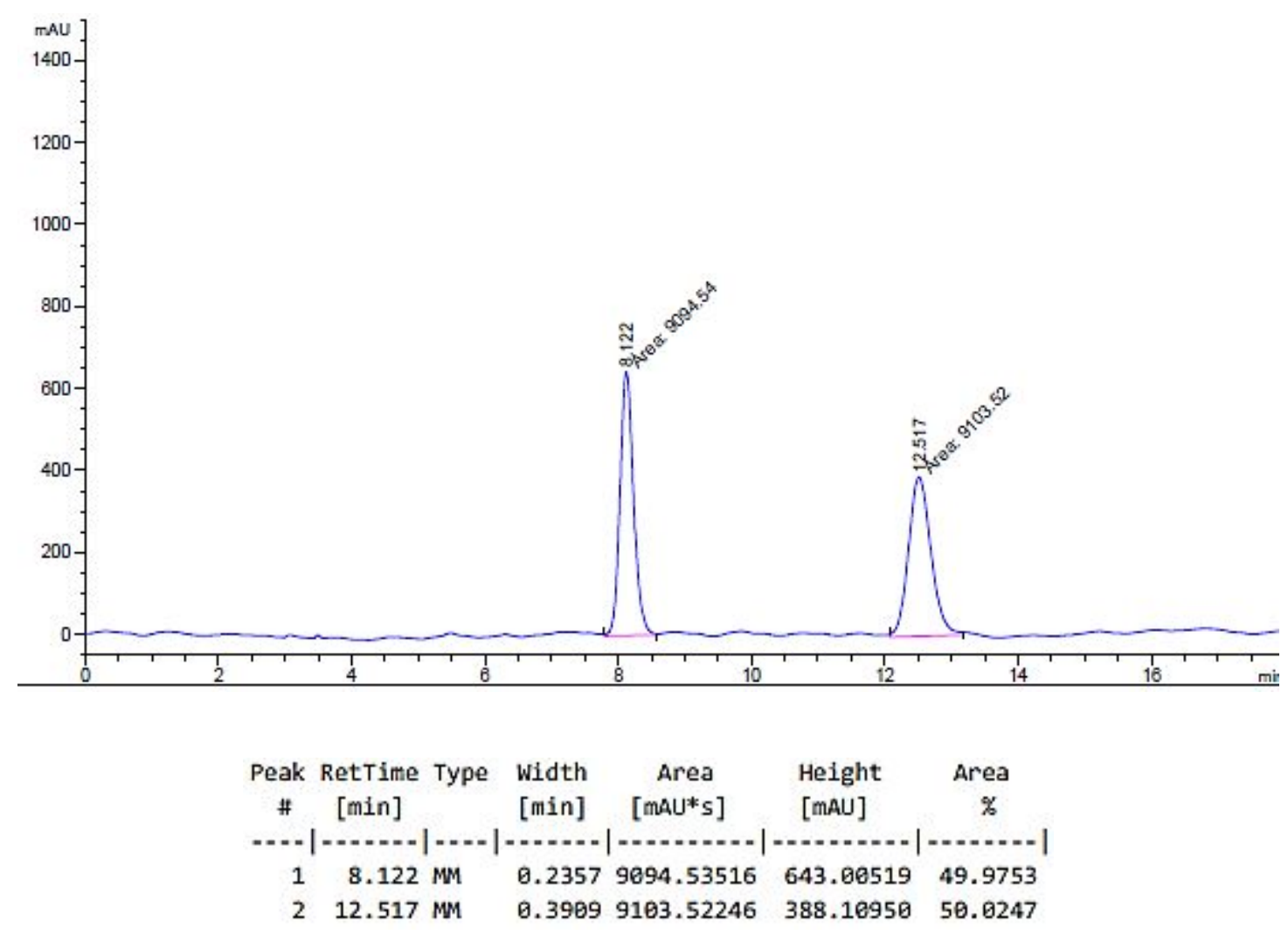

20d-HPLC (93\%)

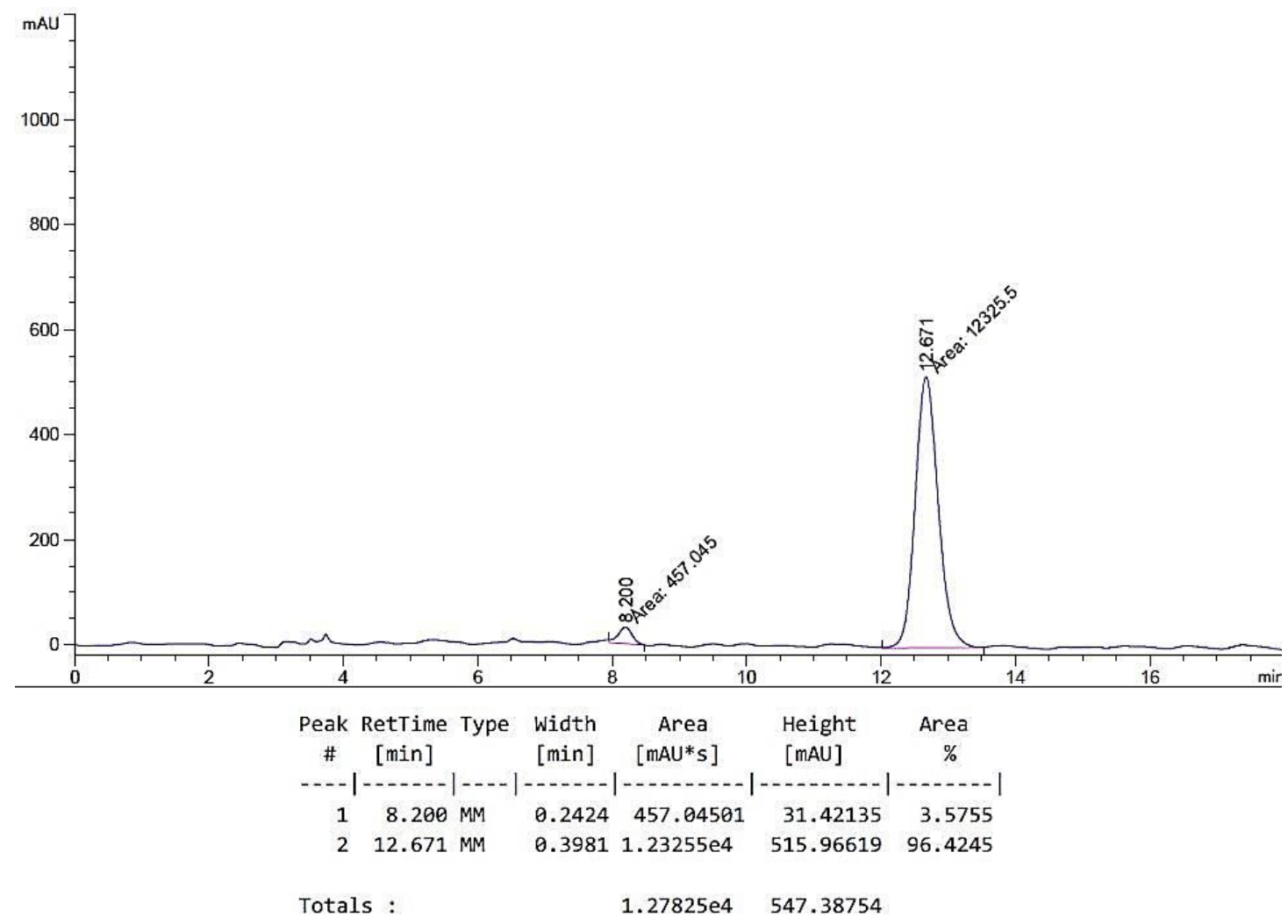

(S,E)-5-((tert-butyldimethylsilyl)oxy)-4,4-difluoro-1,2-diphenylpent-2-en-1-ol (21d) - 


\section{${ }^{1} \mathrm{H}$ NMR}
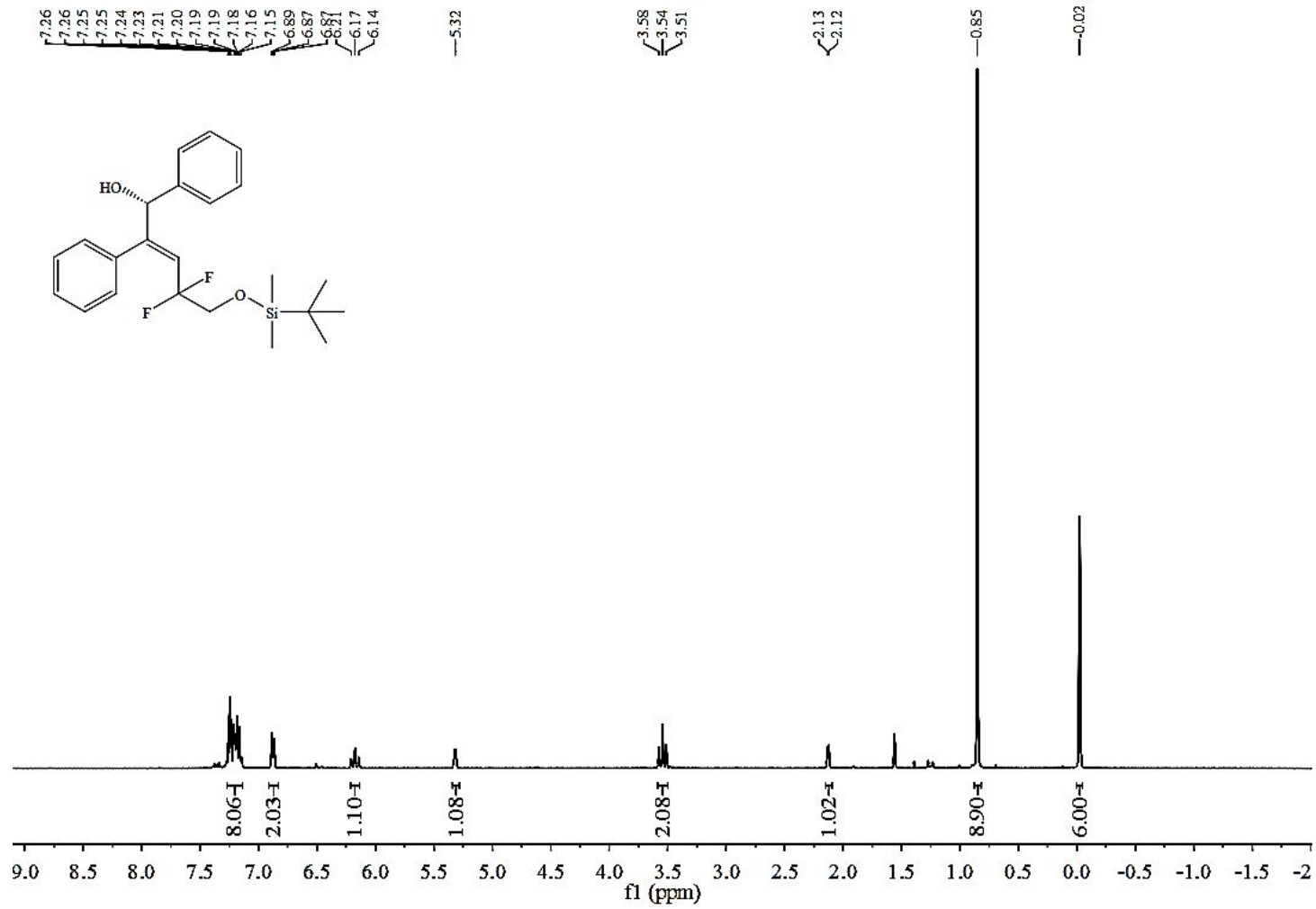

(S,E)-5-((tert-butyldimethylsilyl)oxy)-4,4-difluoro-1,2-diphenylpent-2-en-1-ol (21d) ${ }^{13} \mathrm{C}$ NMR

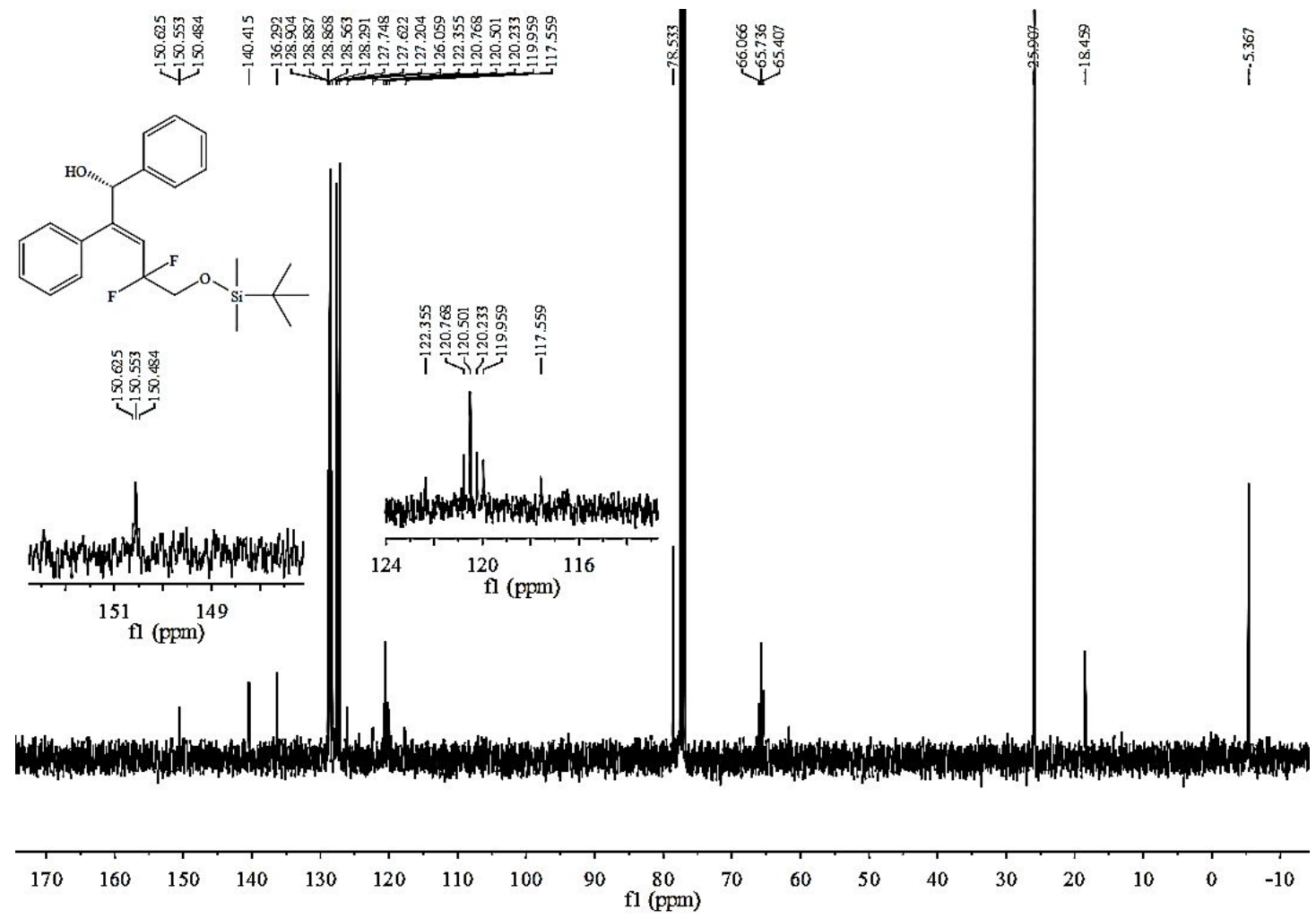

(S,E)-5-((tert-butyldimethylsilyl)oxy)-4,4-difluoro-1,2-diphenylpent-2-en-1-ol (21d) - 


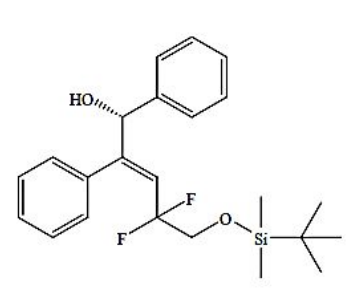

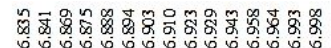

$8 \% 8888808808$

\begin{tabular}{|c|c|c|c|c|c|c|c|c|c|c|}
\hline 80 & 50 & 20 & -10 & -40 & -70 & $\begin{array}{c}-100 \\
\mathrm{fl}(\mathrm{ppm})\end{array}$ & -140 & -180 & -220 & -260 \\
\hline
\end{tabular}

21d-HPLC (racemic)

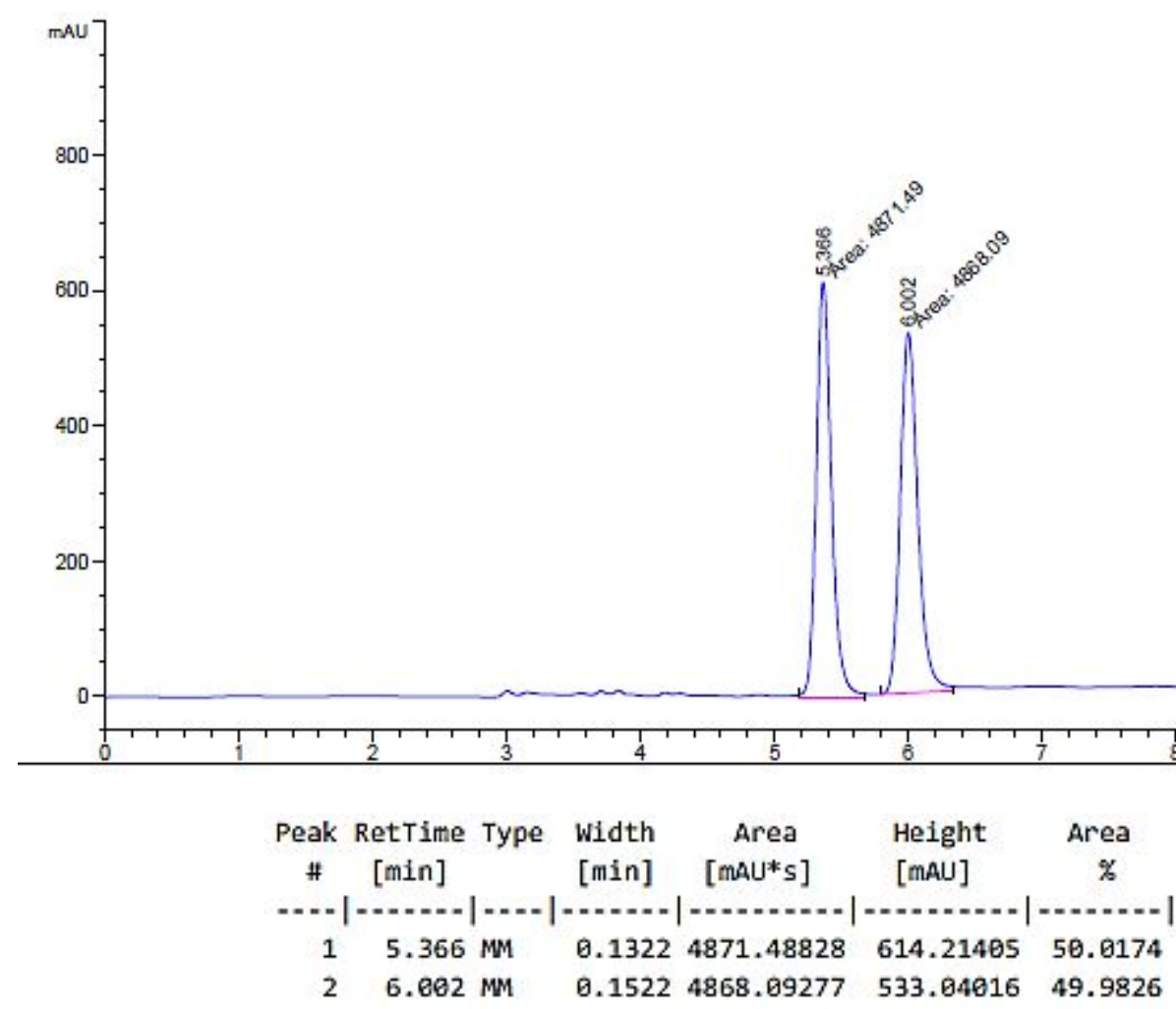

21d-HPLC (86\%) 


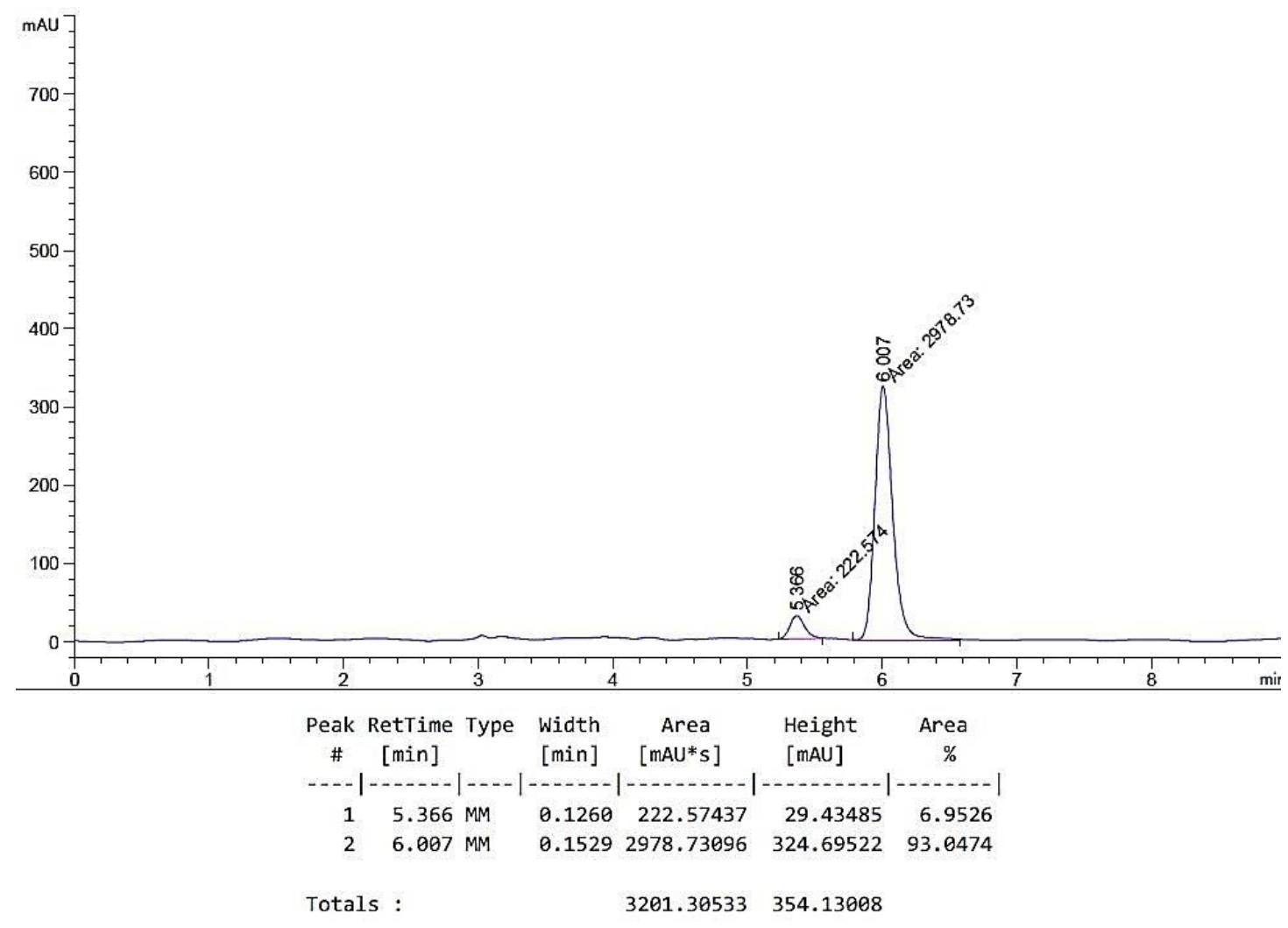

(S,E)-7-((tert-butyldimethylsilyl)oxy)-6,6-difluoro-1,4-diphenylhept-4-en-3-ol (22d) ${ }^{1} \mathrm{H}$ NMR
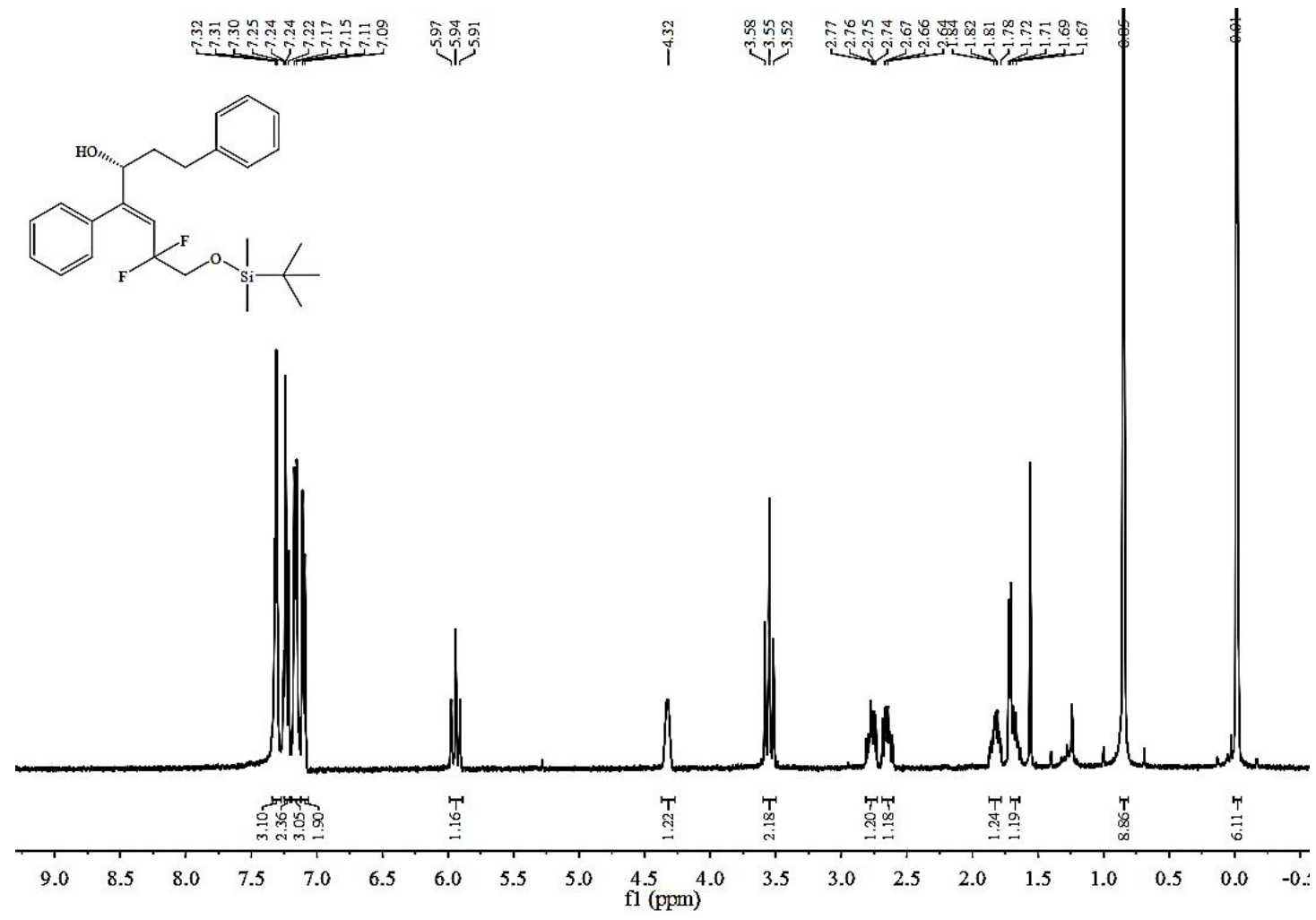

(S,E)-7-((tert-butyldimethylsilyl)oxy)-6,6-difluoro-1,4-diphenylhept-4-en-3-ol (22d) - 
${ }^{13} \mathrm{C}$ NMR
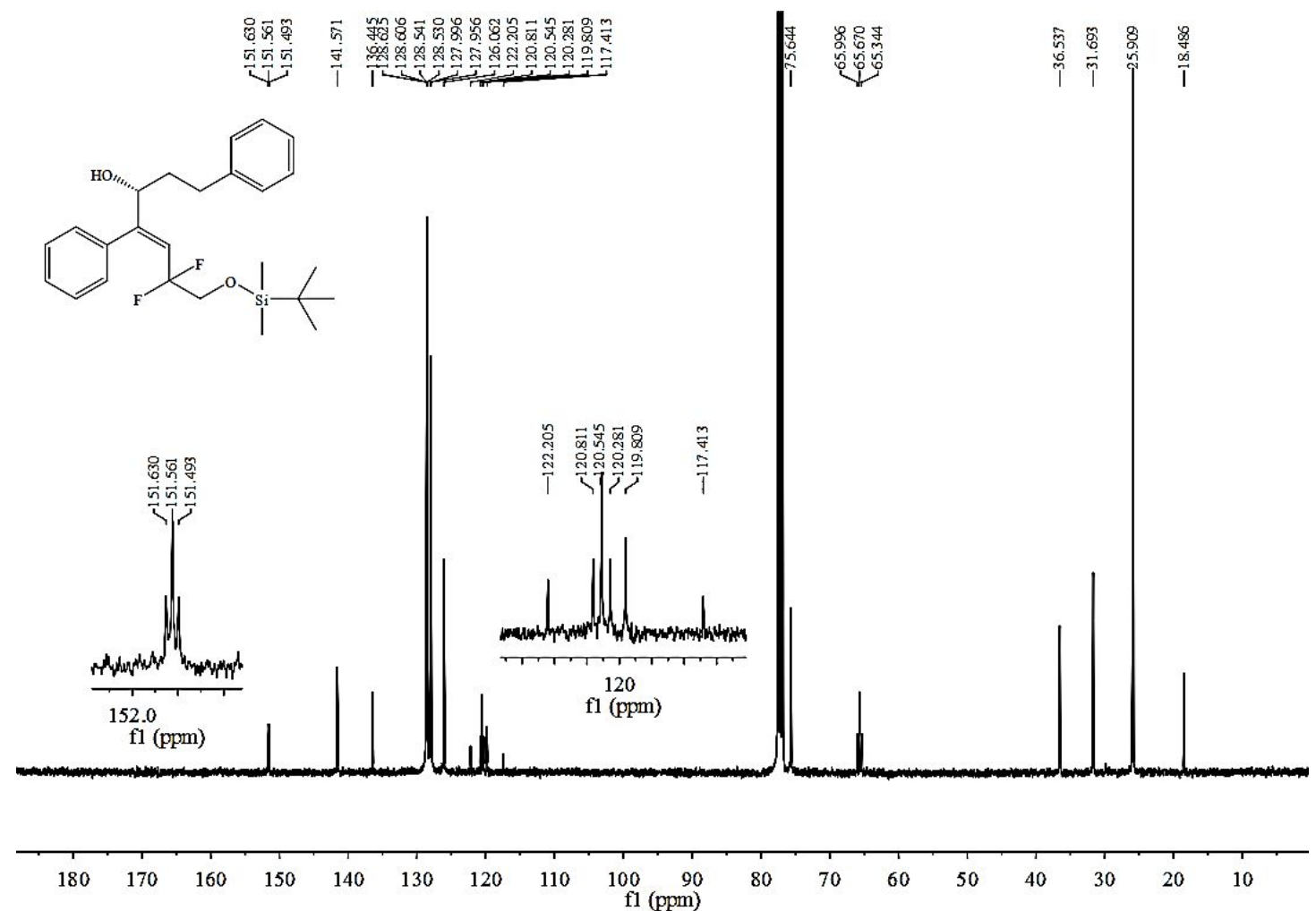

(S,E)-7-((tert-butyldimethylsilyl)oxy)-6,6-difluoro-1,4-diphenylhept-4-en-3-ol (22d) $-{ }^{19} \mathrm{~F}$ NMR

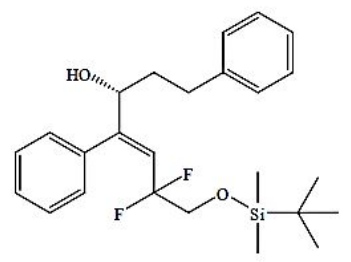

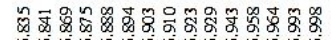

$8.88 \% 8888888 \%$

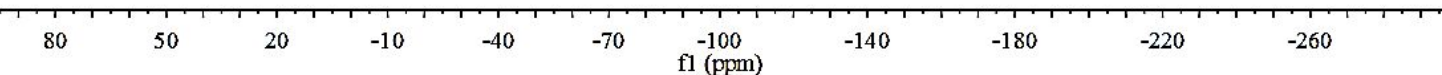

22d-HPLC (racemic) 


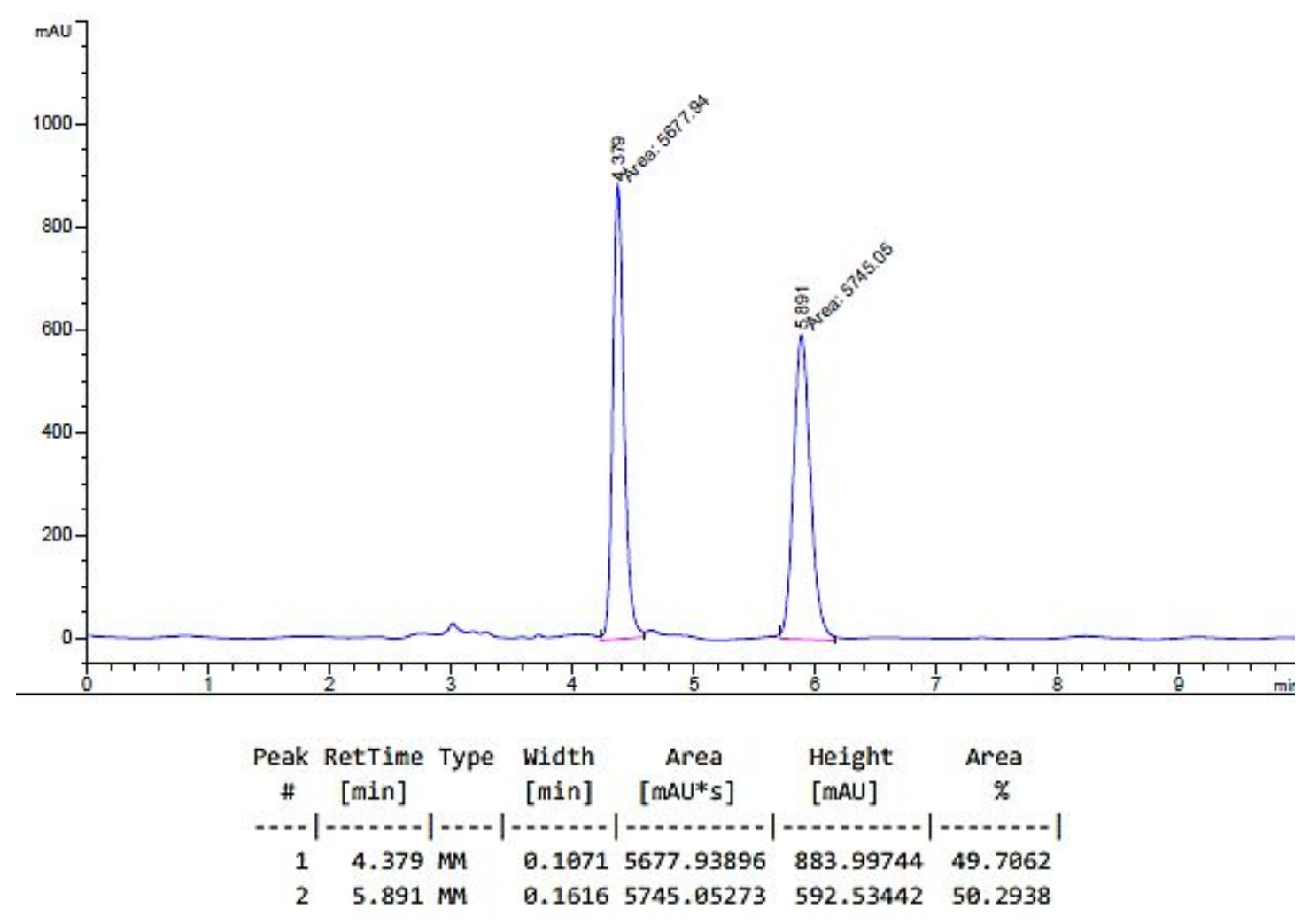

22d-HPLC (95\%)

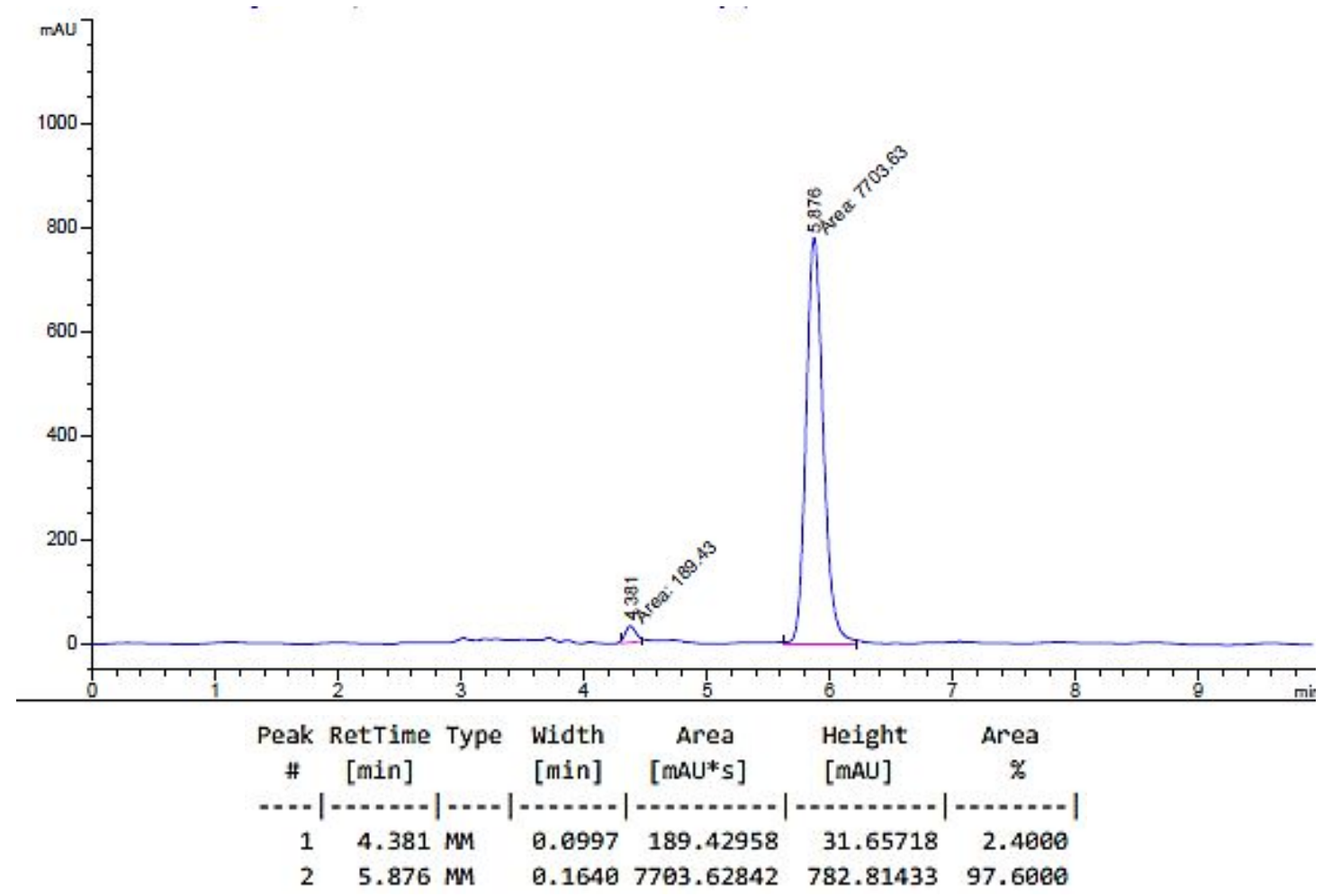

(S,E)-1-((tert-butyldimethylsilyl)oxy)-2,2-difluoro-4-phenyldec-3-en-5-ol (23d) - ${ }^{1} \mathrm{H}$ 


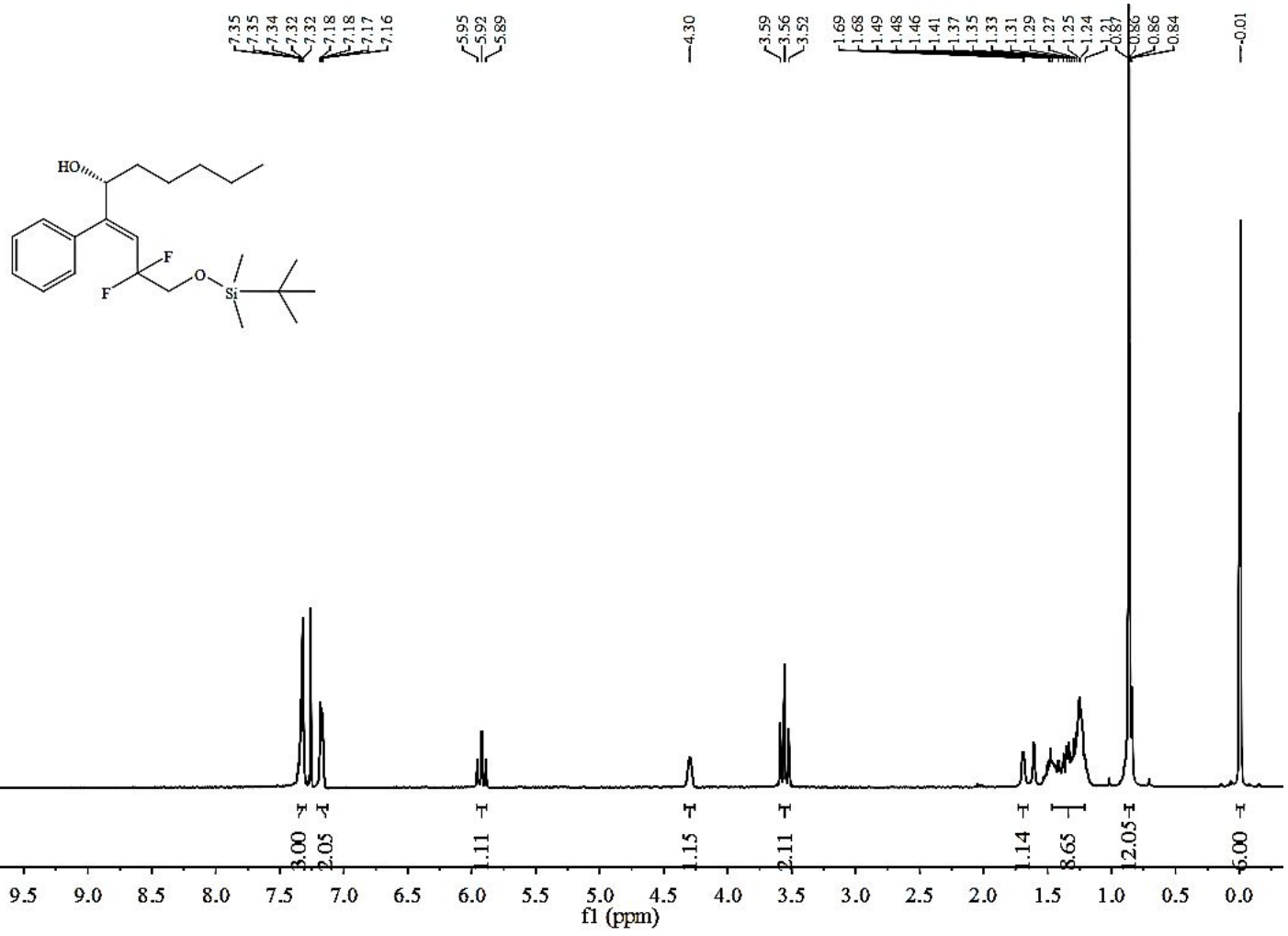

(S,E)-1-((tert-butyldimethylsilyl)oxy)-2,2-difluoro-4-phenyldec-3-en-5-ol (23d) $-{ }^{13} \mathrm{C}$ NMR
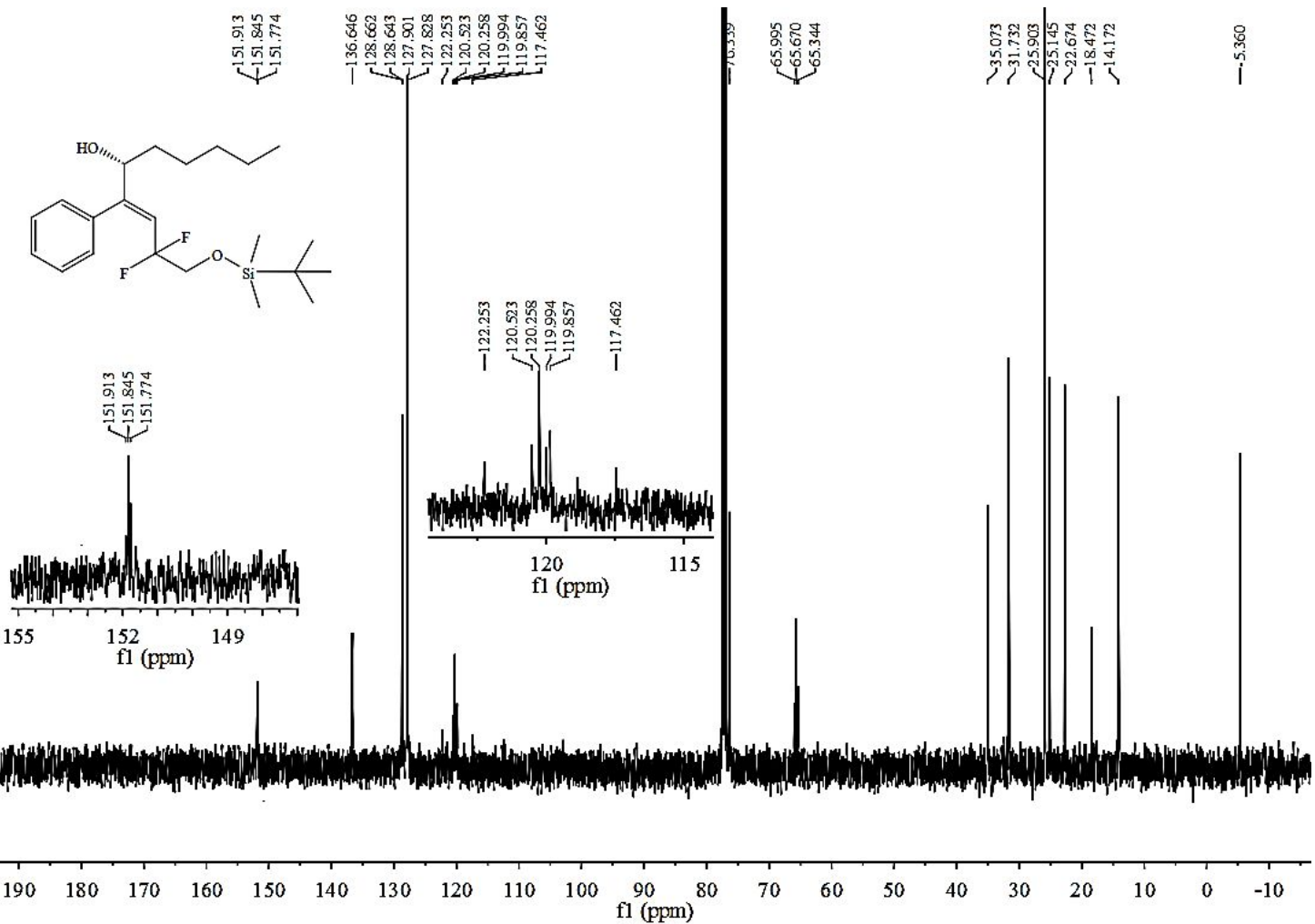

(S,E)-1-((tert-butyldimethylsilyl)oxy)-2,2-difluoro-4-phenyldec-3-en-5-ol (23d) 
$-{ }^{19}$ F NMR
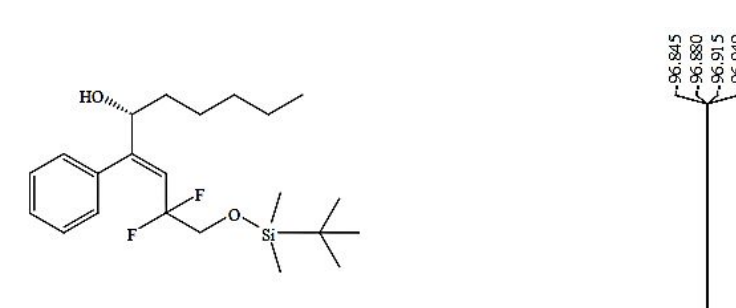

\begin{tabular}{|c|c|c|c|c|c|c|c|c|c|}
\hline 90 & 60 & 30 & 0 & -30 & -70 & f1 (ppm) & -160 & -210 & -260 \\
\hline
\end{tabular}

23d-HPLC (racemic)

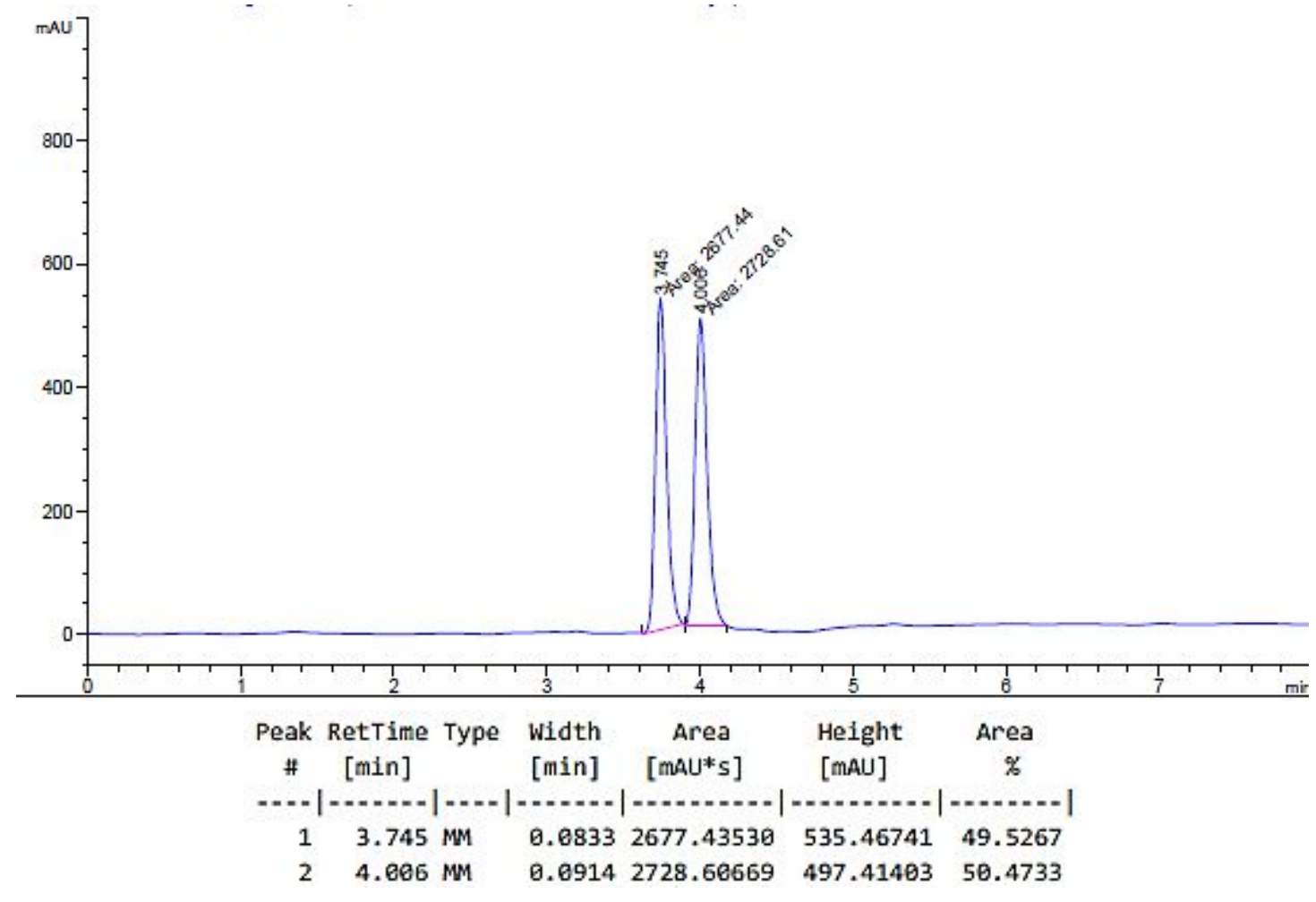

23d-HPLC (96\%) 


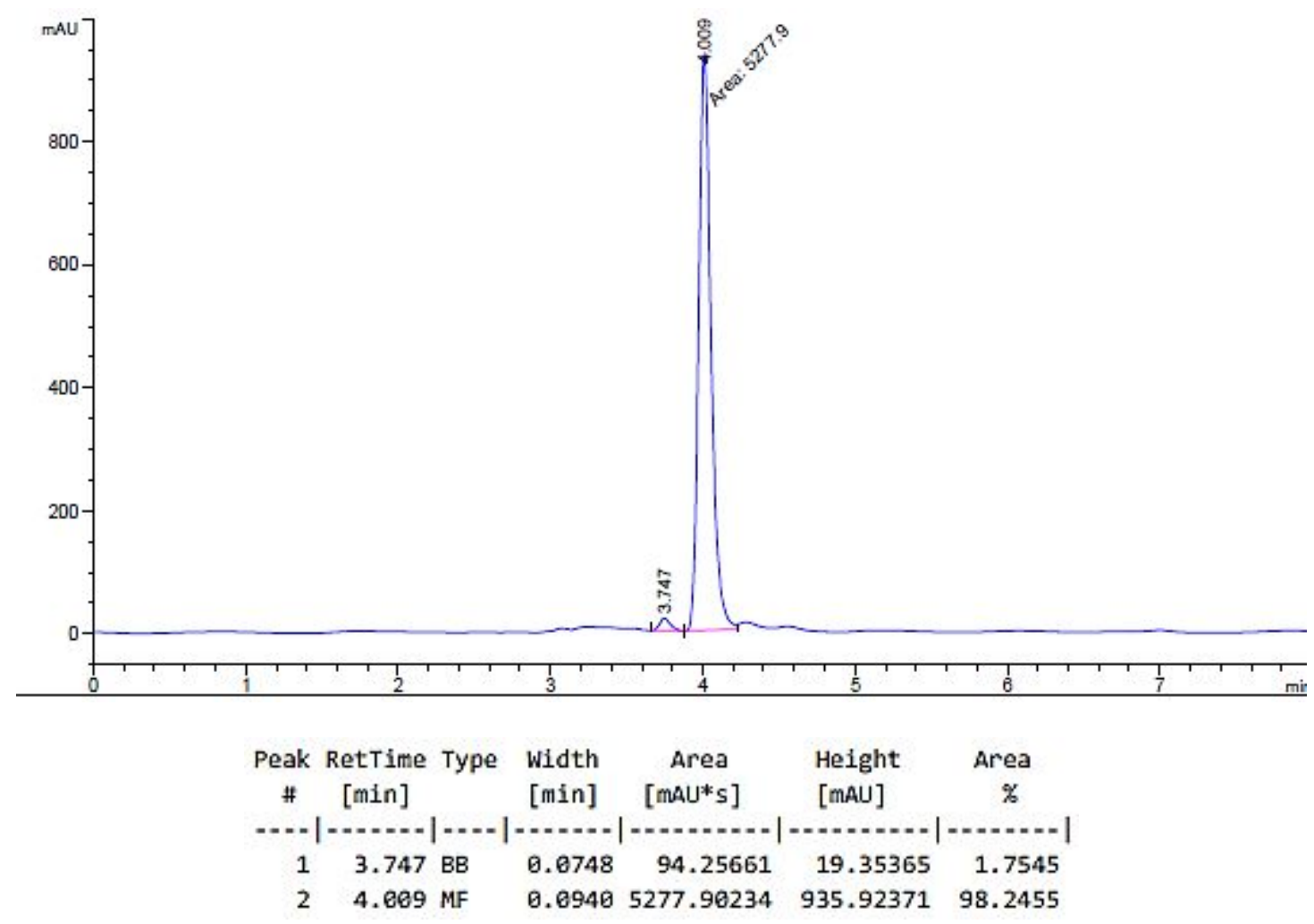

(S,E)-7-(benzyloxy)-6,6-difluoro-1,4-diphenylhept-4-en-3-ol (24d) - ${ }^{1} \mathrm{H}$ NMR

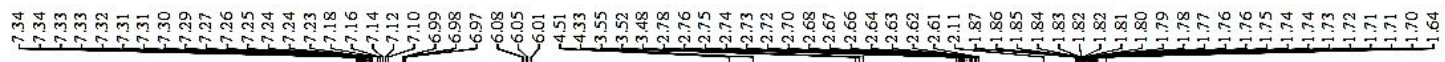

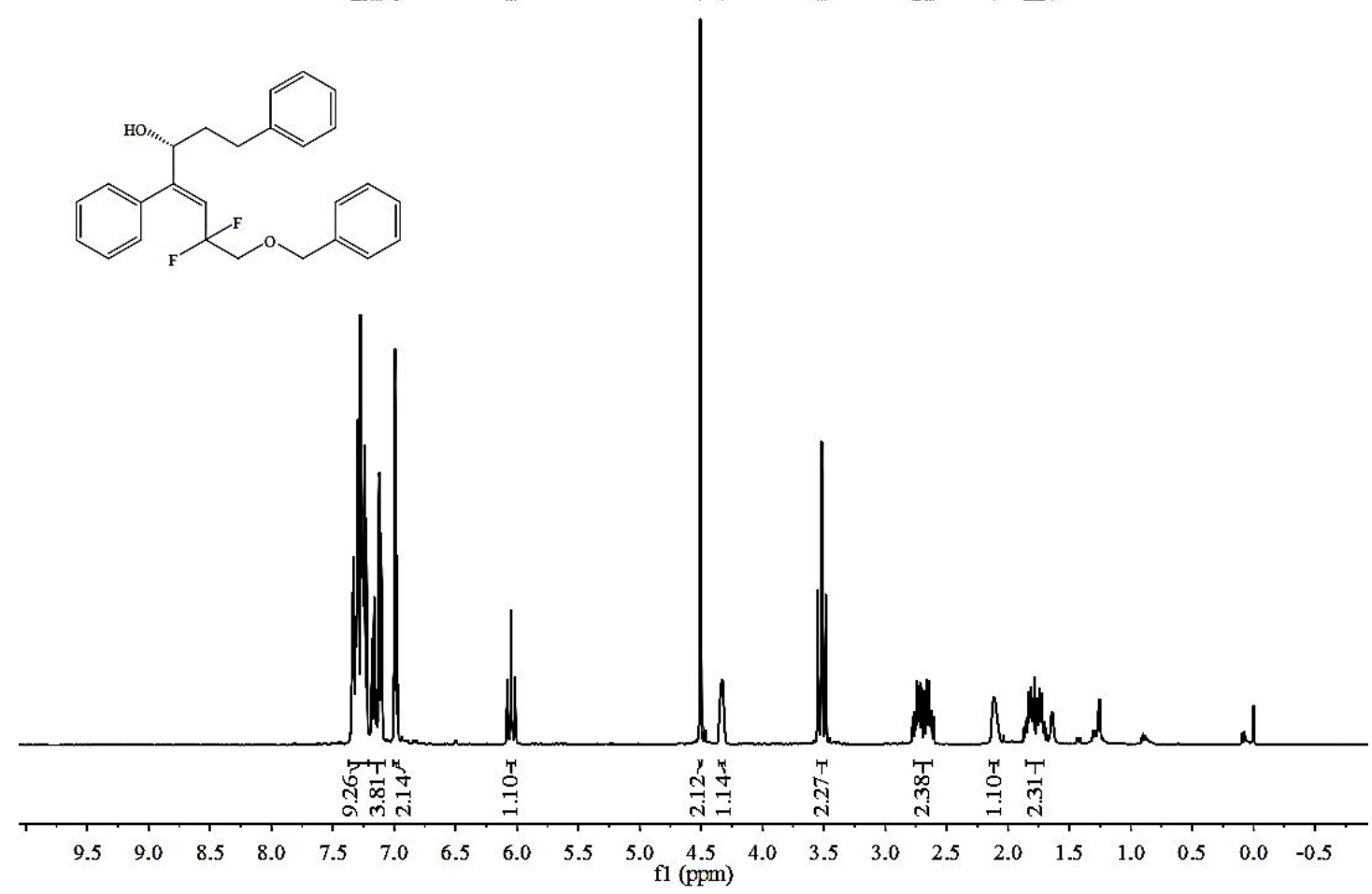

(S,E)-7-(benzyloxy)-6,6-difluoro-1,4-diphenylhept-4-en-3-ol (24d) $-{ }^{13} \mathrm{C}$ NMR 


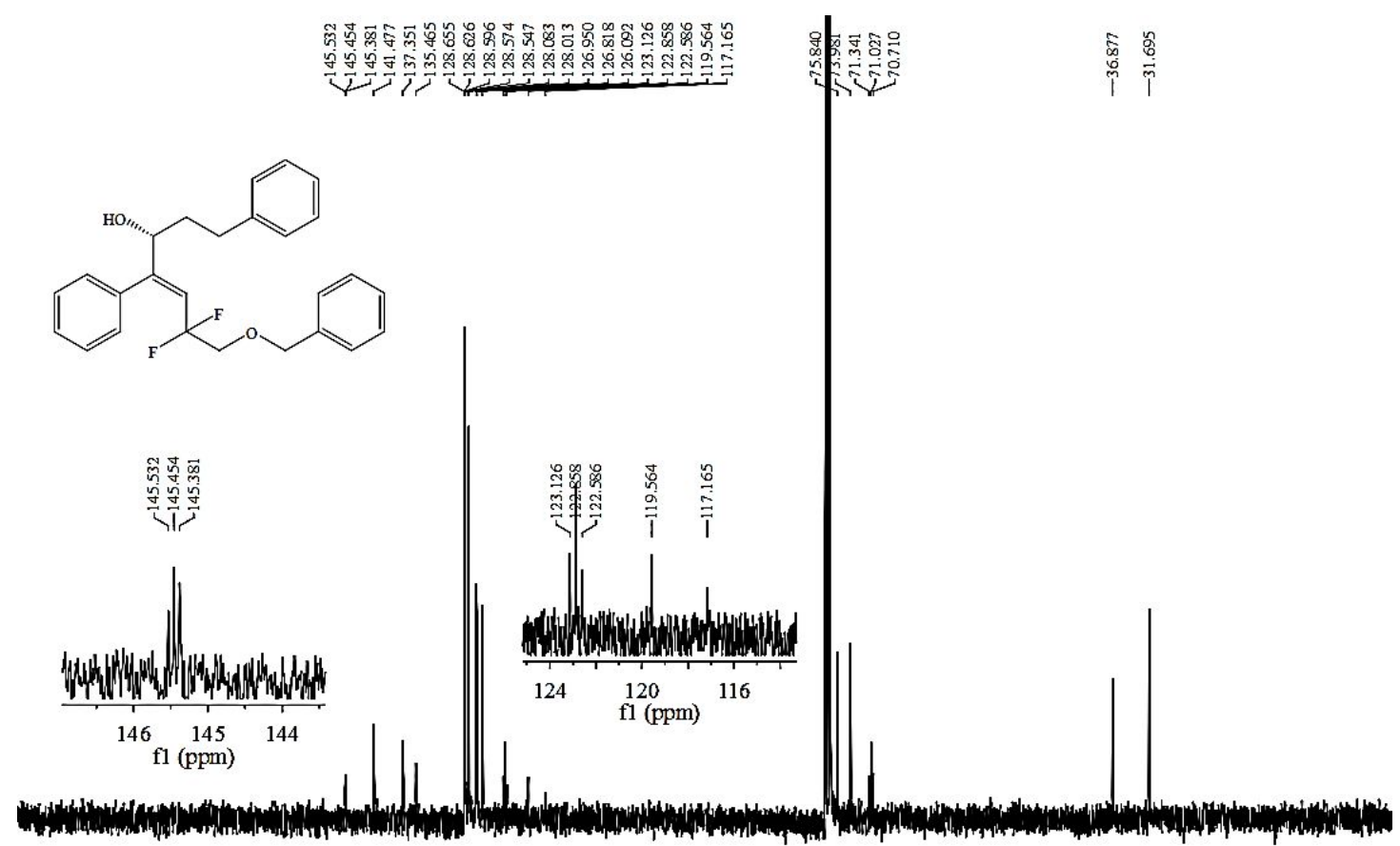

(S,E)-7-(benzyloxy)-6,6-difluoro-1,4-diphenylhept-4-en-3-ol (24d) - ${ }^{19}$ F NMR
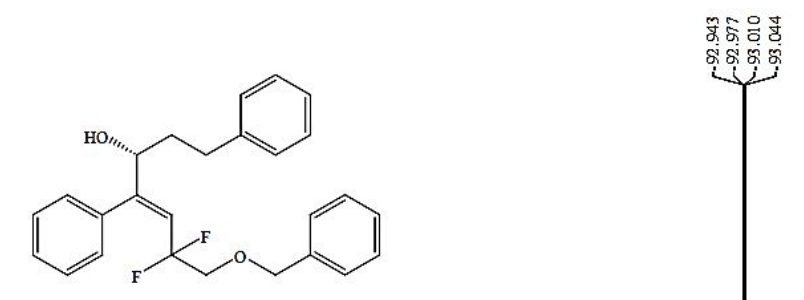

24d-HPLC (racemic) 


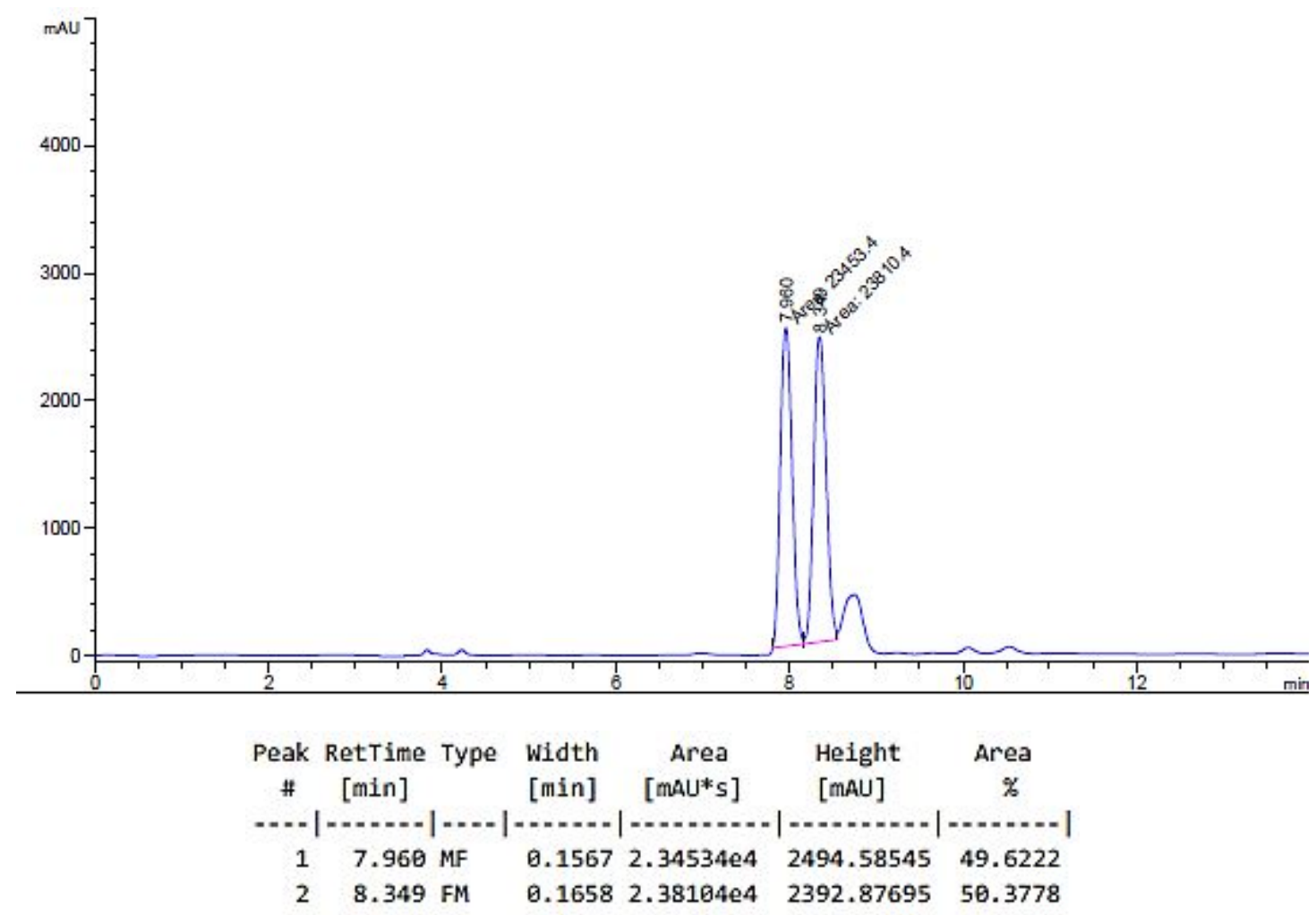

24d-HPLC (94\%)

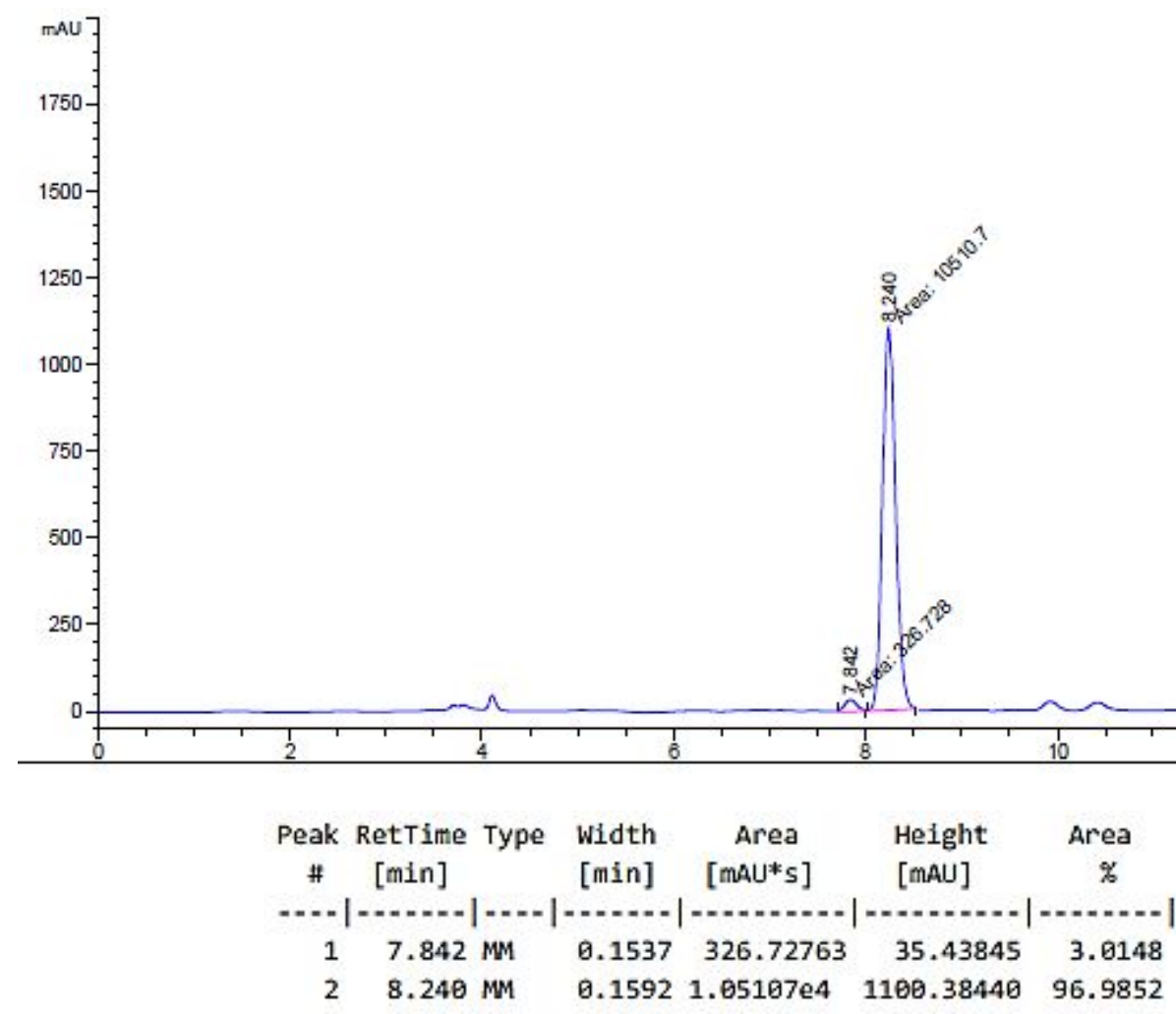

(S,E)-1-(benzyloxy)-2,2-difluoro-4-phenyldec-3-en-5-ol (25d) - ${ }^{1} \mathrm{H}$ NMR 


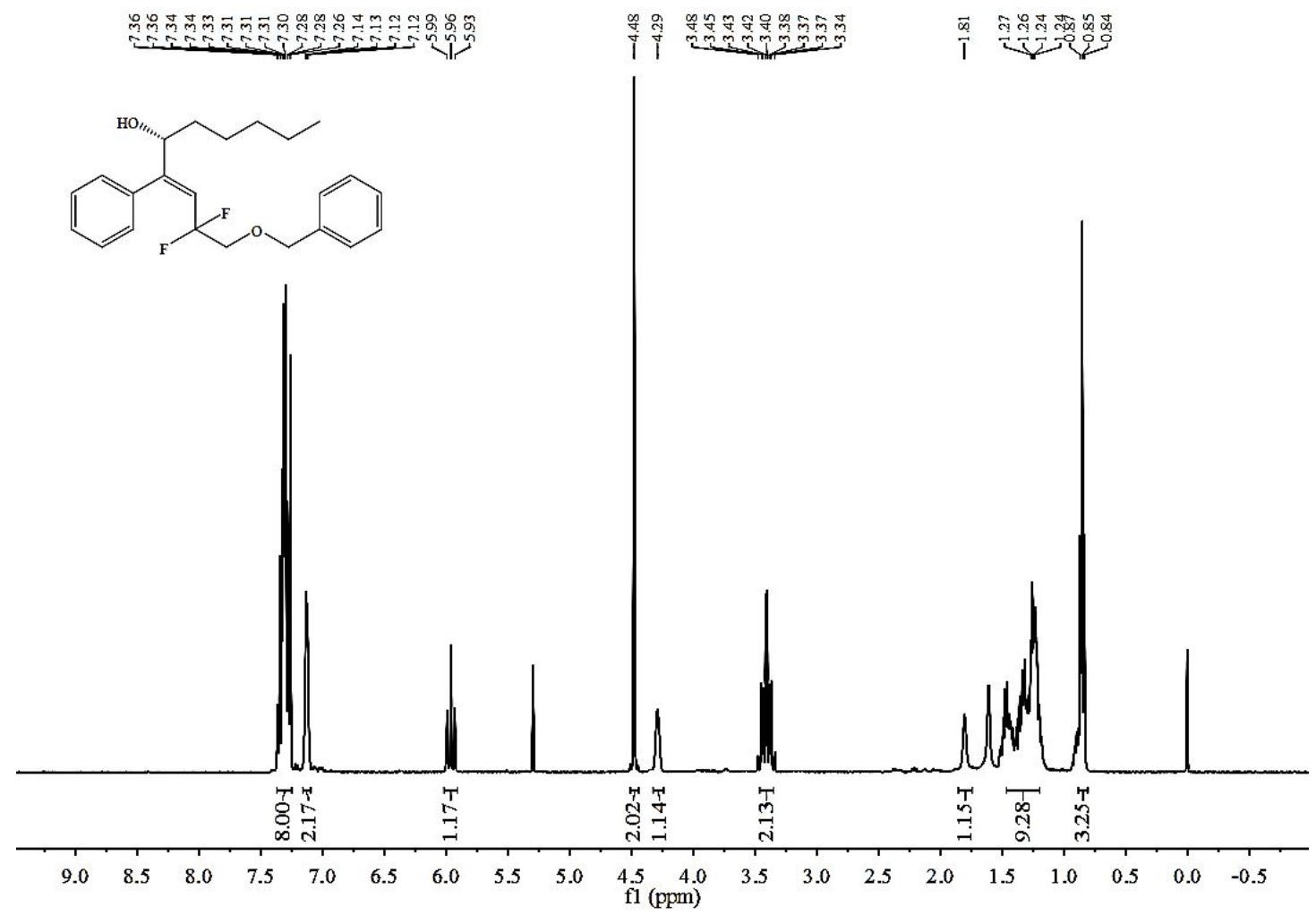

(S,E)-1-(benzyloxy)-2,2-difluoro-4-phenyldec-3-en-5-ol (25d) - ${ }^{13}$ C NMR

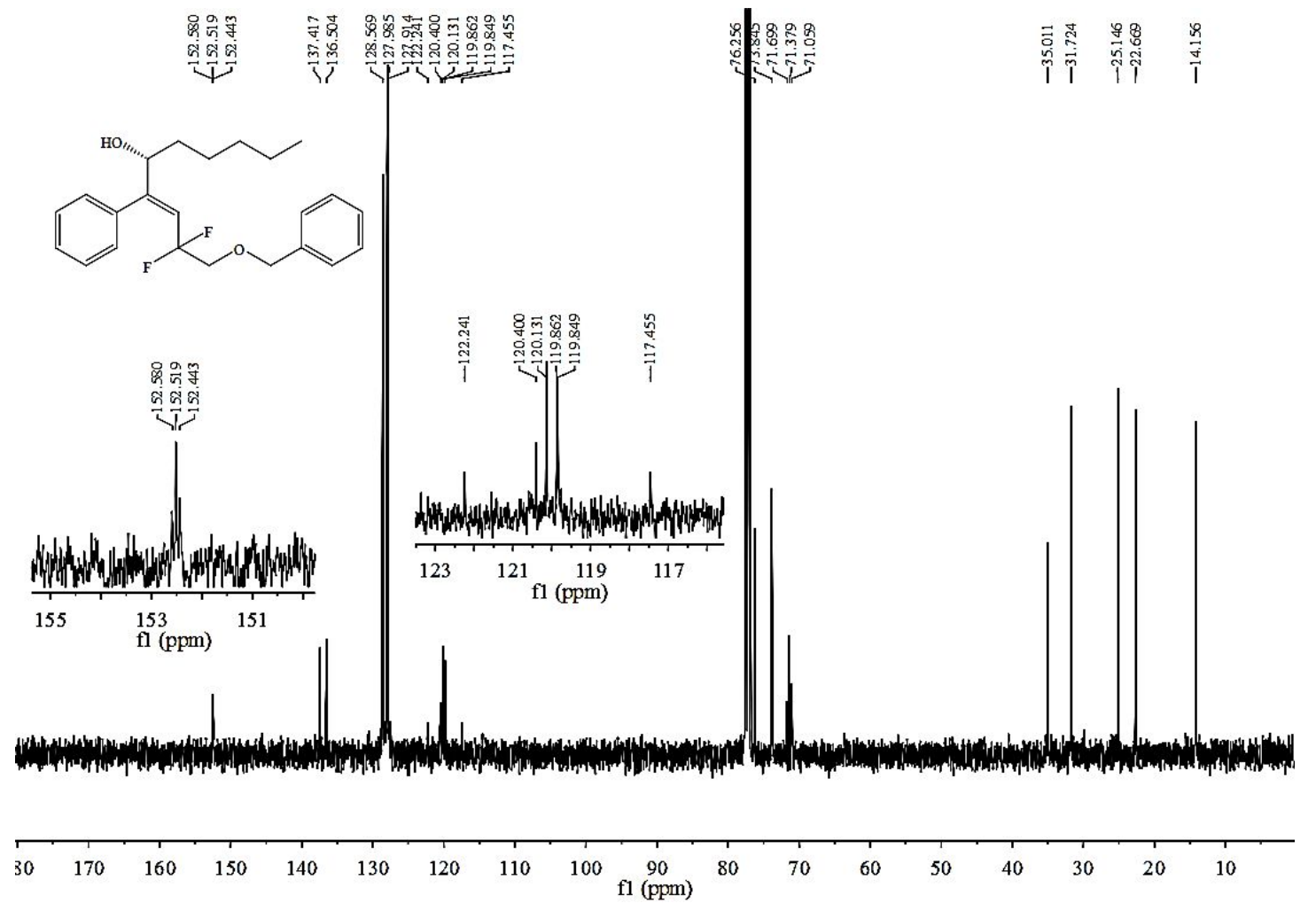

(S,E)-1-(benzyloxy)-2,2-difluoro-4-phenyldec-3-en-5-ol (25d) - ${ }^{19}$ F NMR 


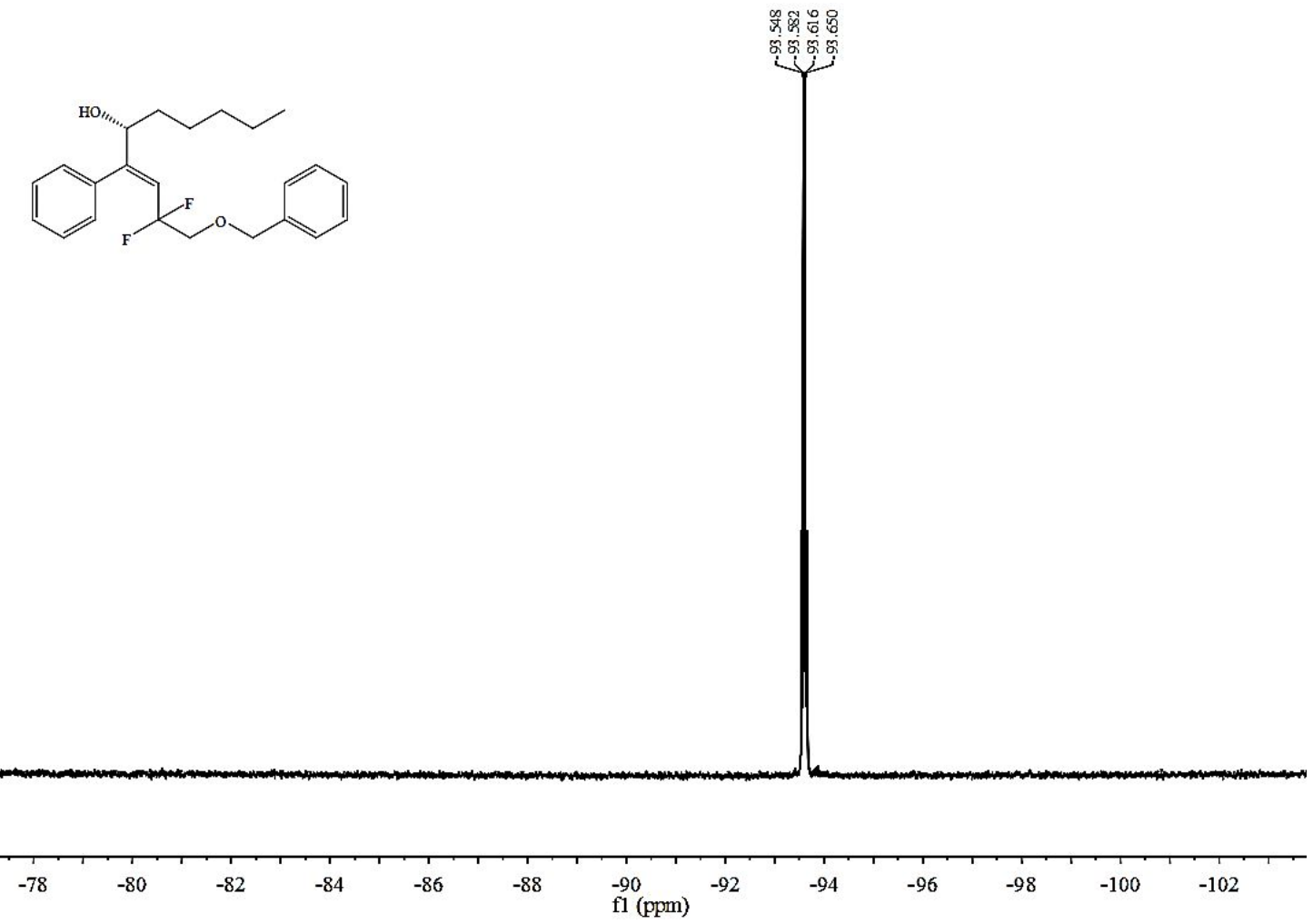

25d-HPLC (racemic)

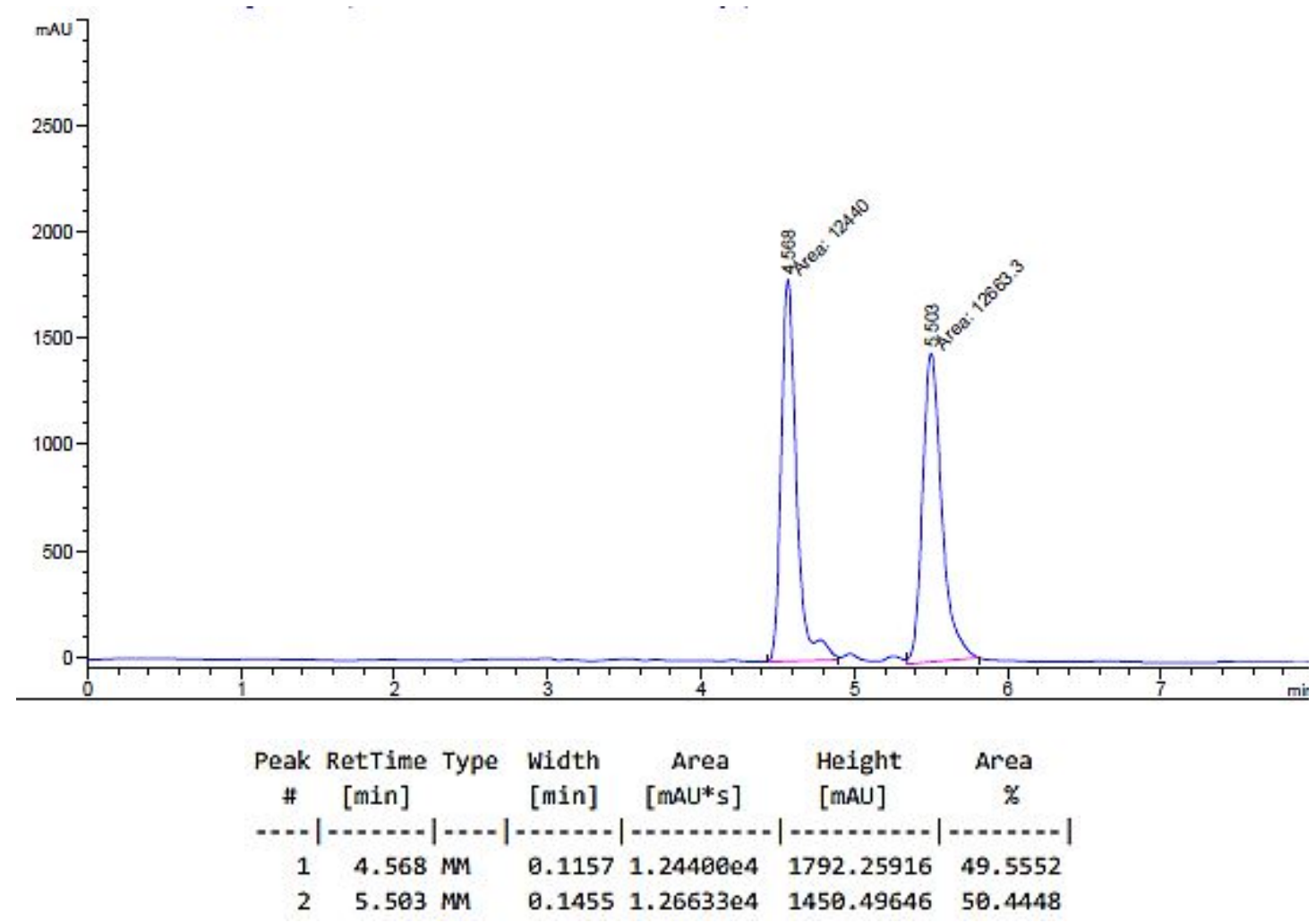

25d-HPLC (95\%) 


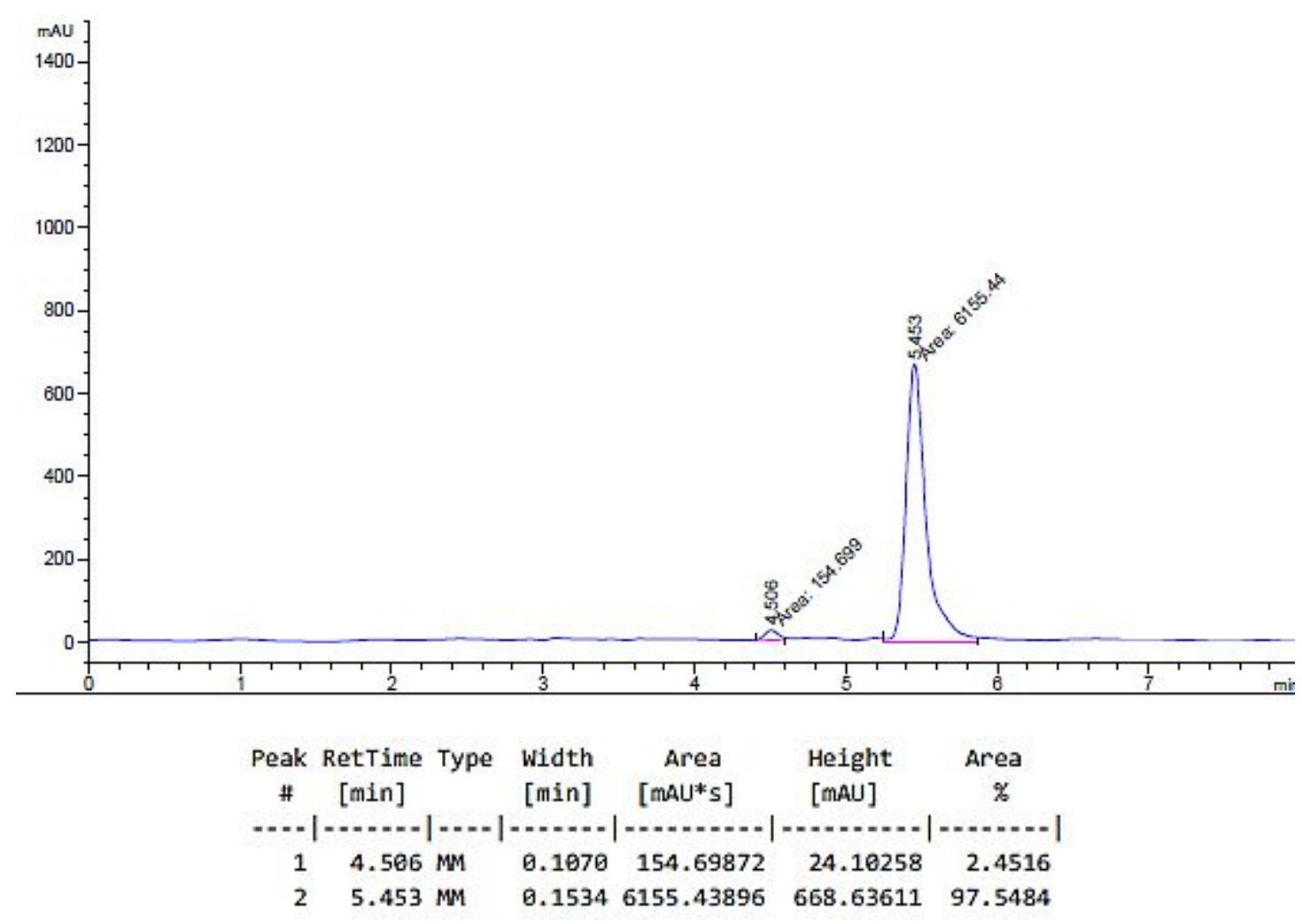

(S,Z)-6-methyl-1-phenyl-4-(thiophen-2-yl)hept-4-en-3-ol (26d) - ${ }^{1} \mathrm{H}$ NMR

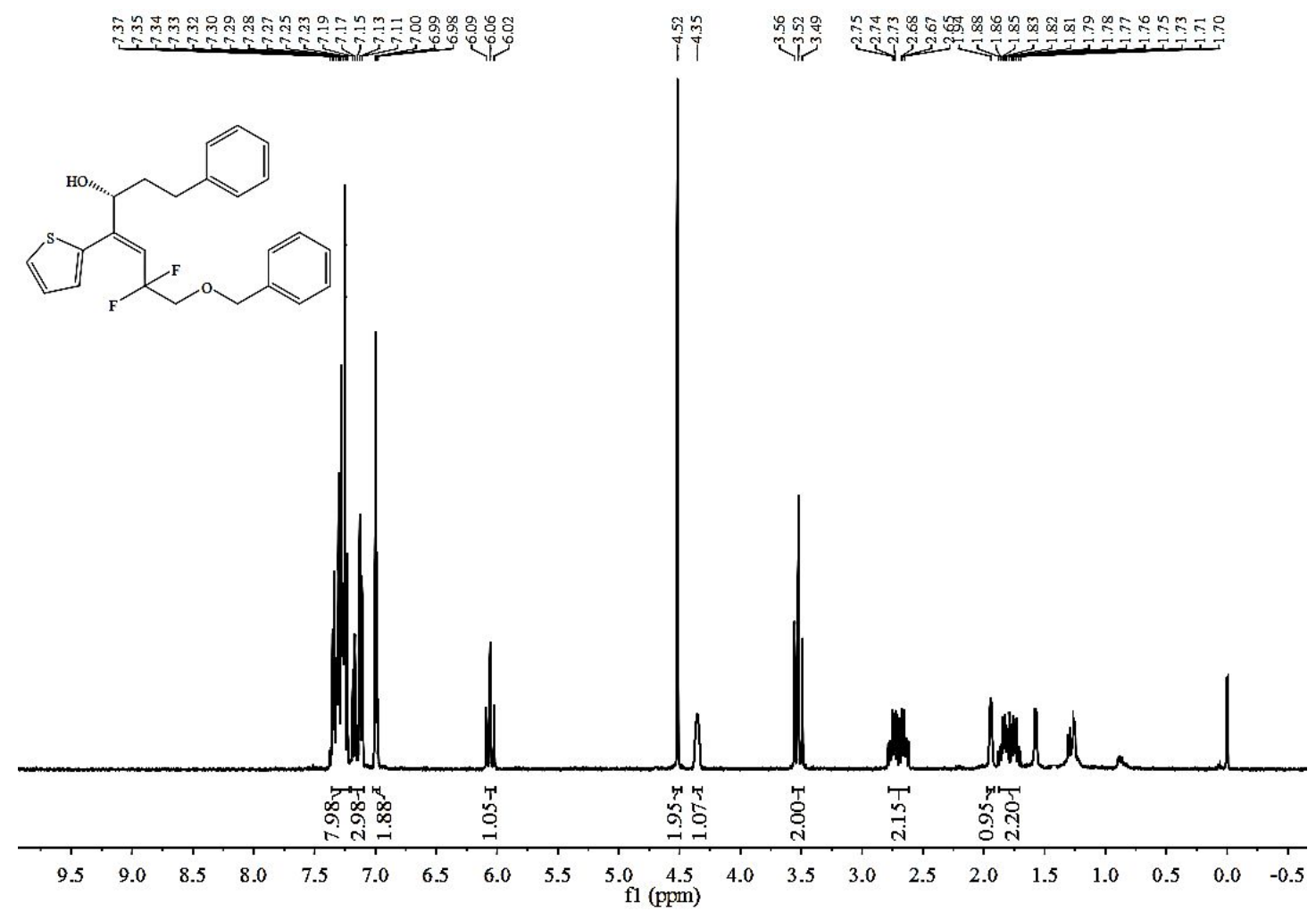

(S,Z)-6-methyl-1-phenyl-4-(thiophen-2-yl)hept-4-en-3-ol (26d) - ${ }^{13} \mathrm{C}$ NMR 


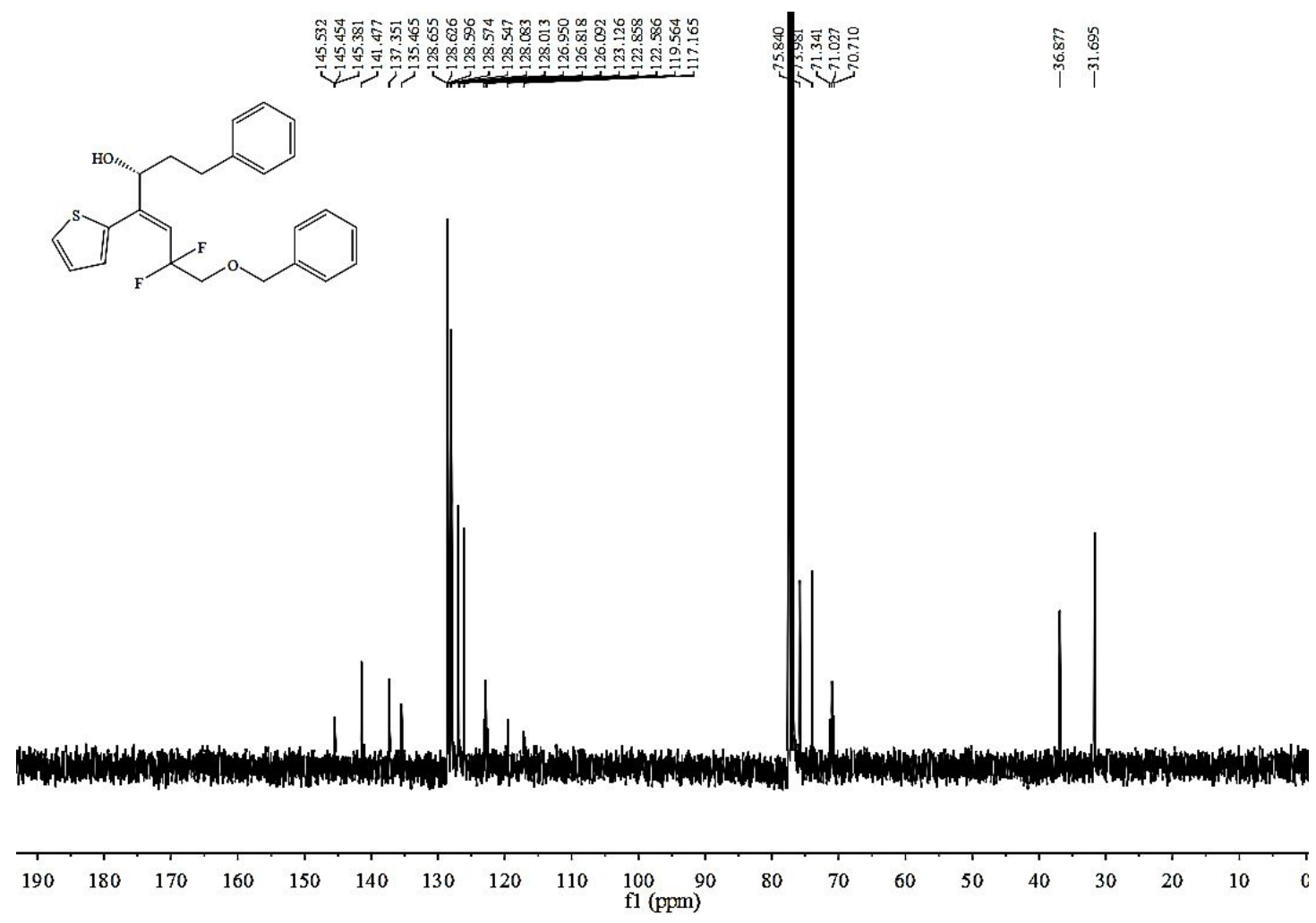

(S,Z)-6-methyl-1-phenyl-4-(thiophen-2-yl)hept-4-en-3-ol (26d) - ${ }^{19}$ F NMR
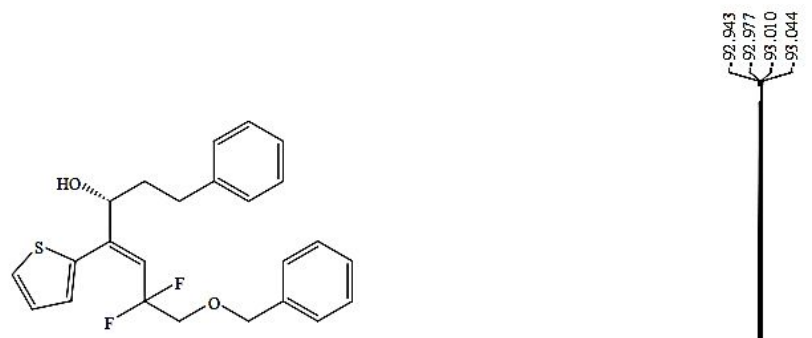

26d-HPLC (racemic) 


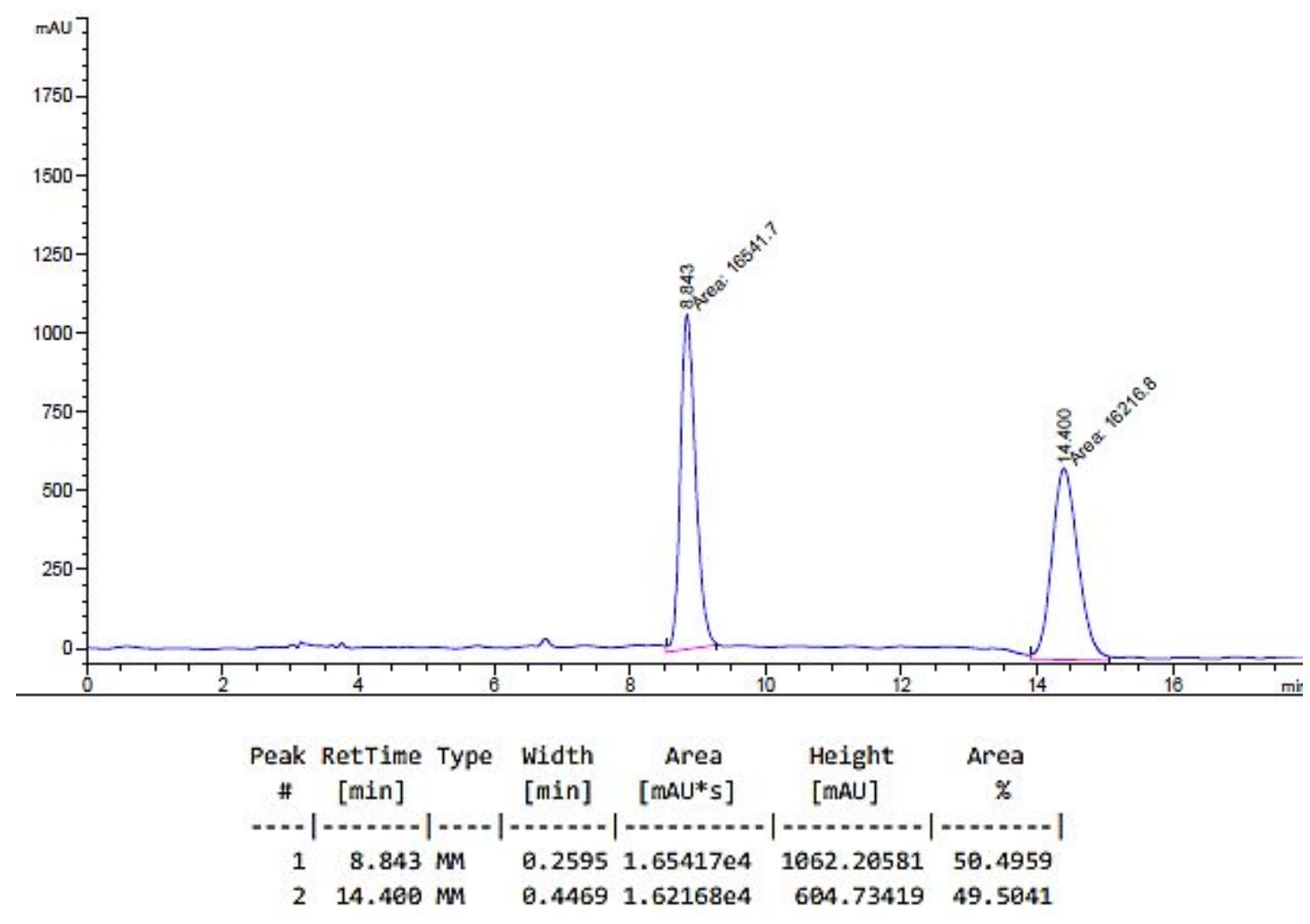

26d-HPLC (95\%)

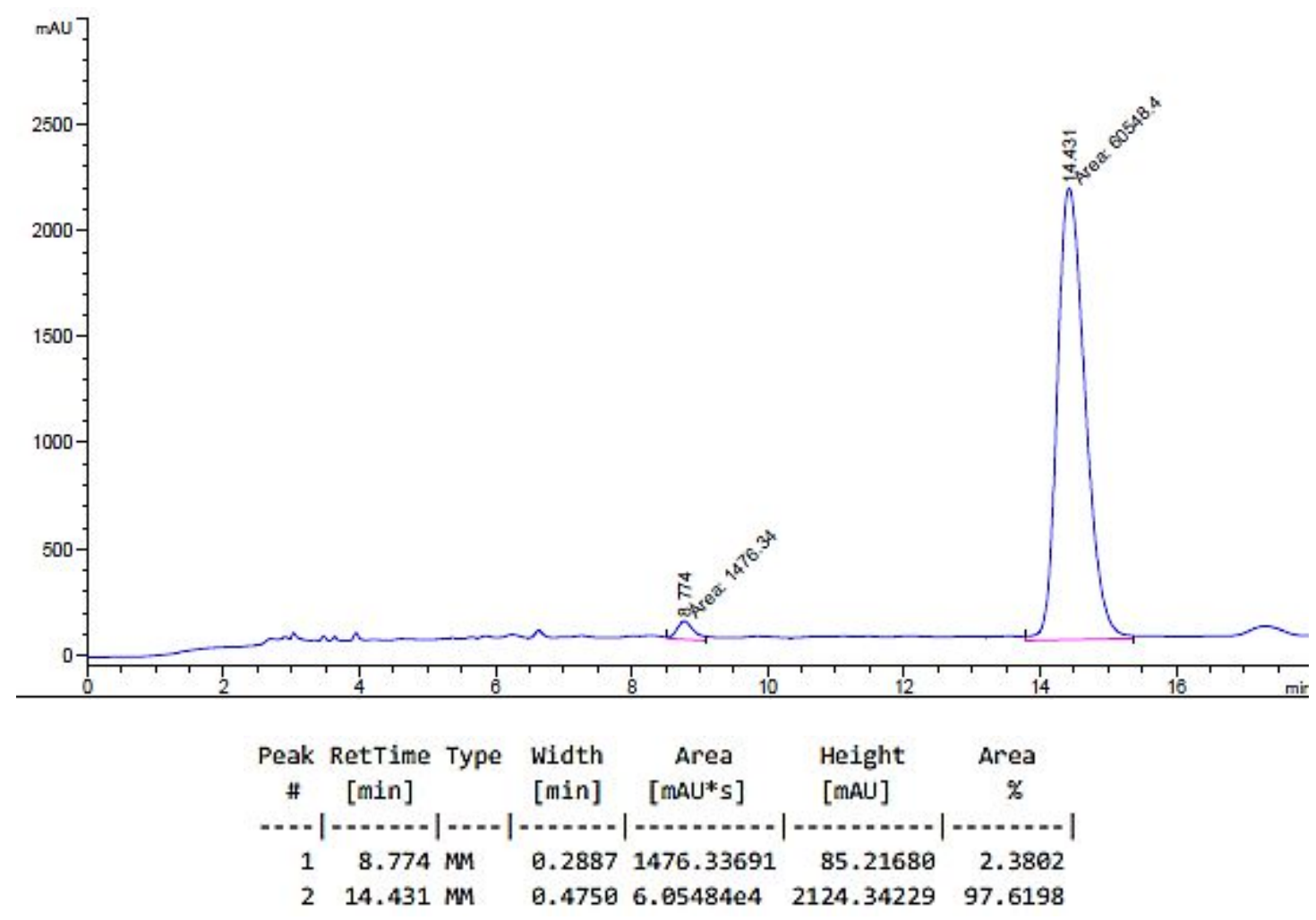

(S,E)-7-(benzyloxy)-6,6-difluoro-1-phenyl-4-(p-tolyl)hept-4-en-3-ol (27d) - ${ }^{1} \mathrm{H}$ NMR 

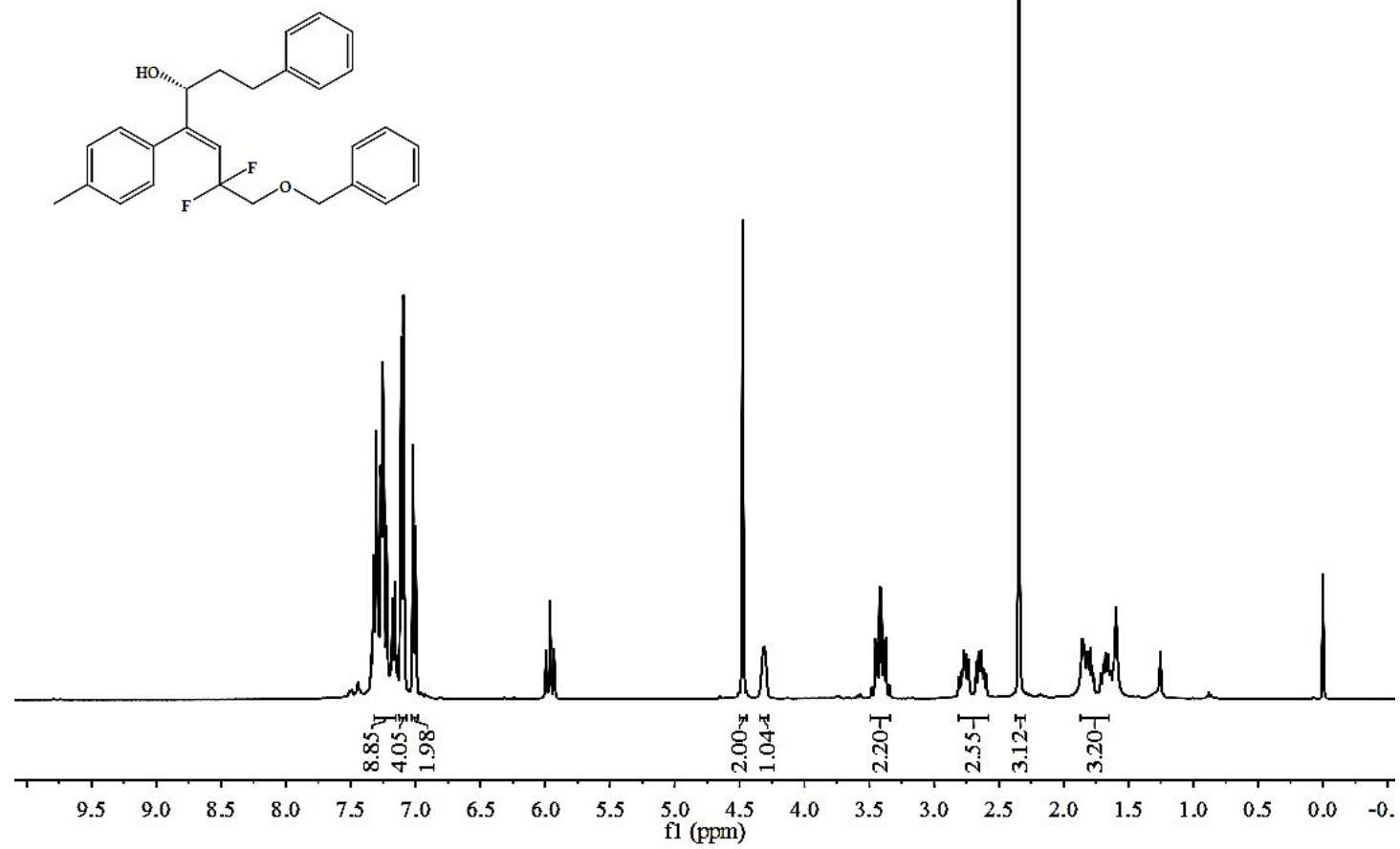

(S,E)-7-(benzyloxy)-6,6-difluoro-1-phenyl-4-(p-tolyl)hept-4-en-3-ol (27d) - ${ }^{13}$ C NMR

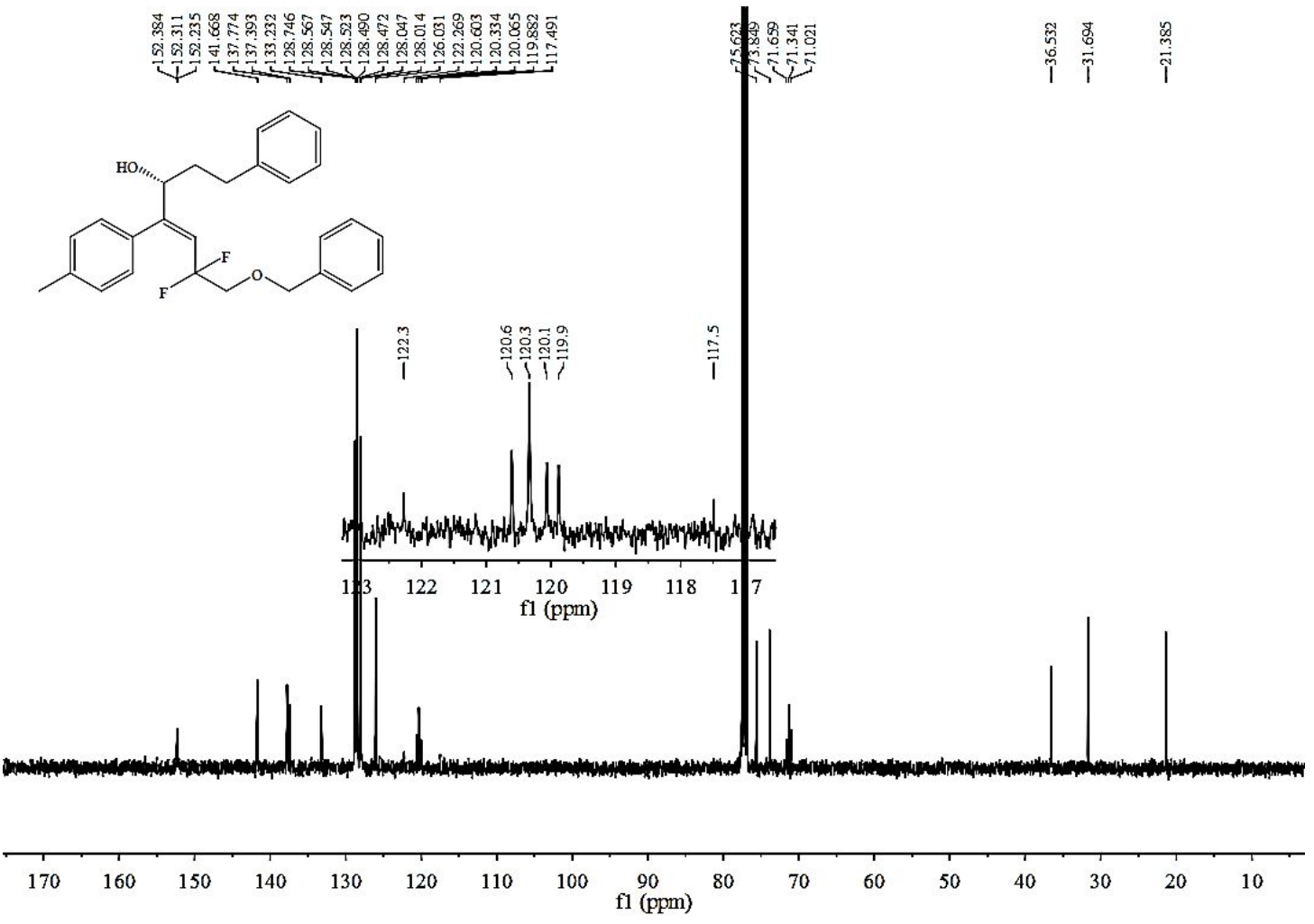

(S,E)-7-(benzyloxy)-6,6-difluoro-1-phenyl-4-(p-tolyl)hept-4-en-3-ol (27d) - ${ }^{19}$ F NMR 


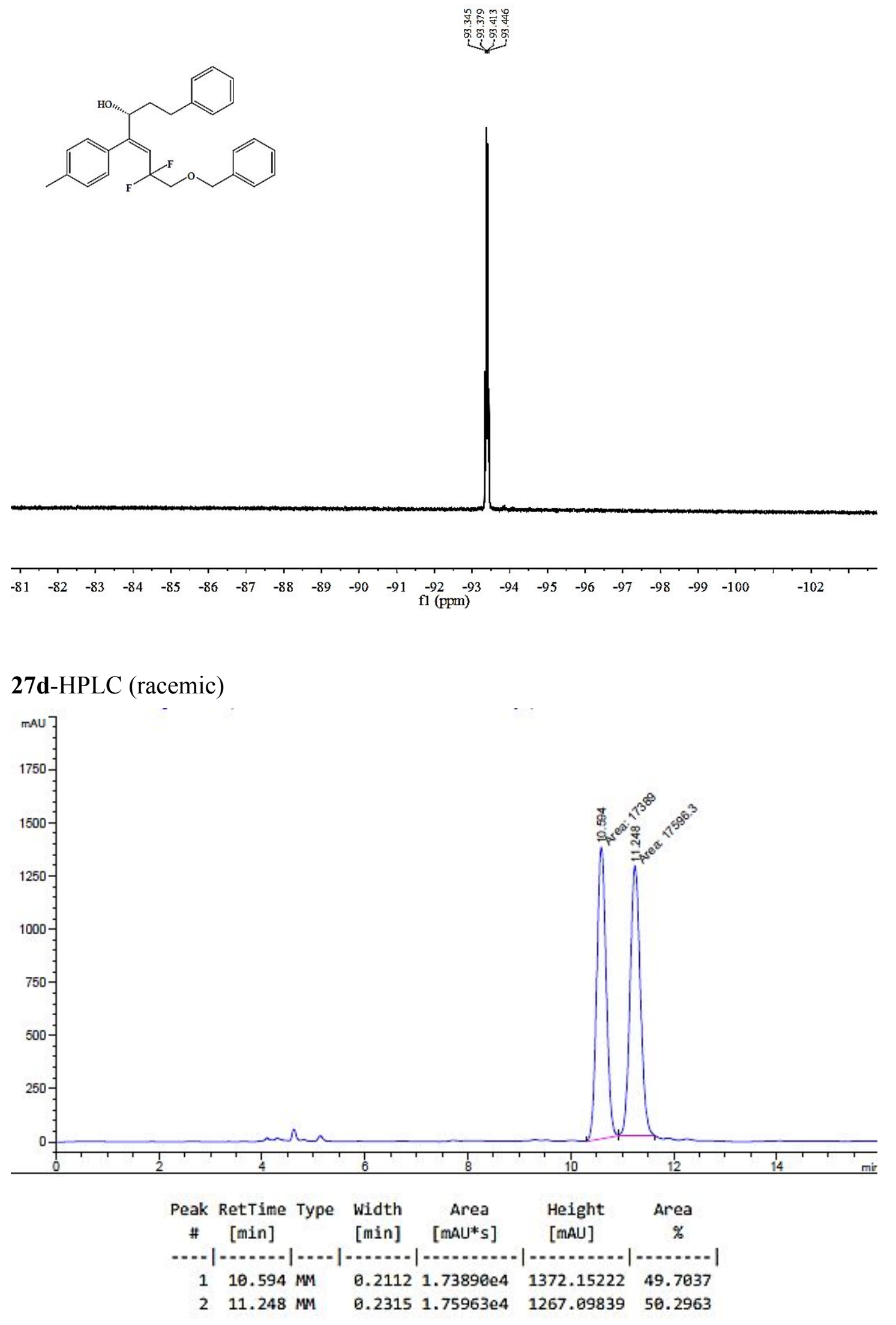

27d-HPLC (94\%) 


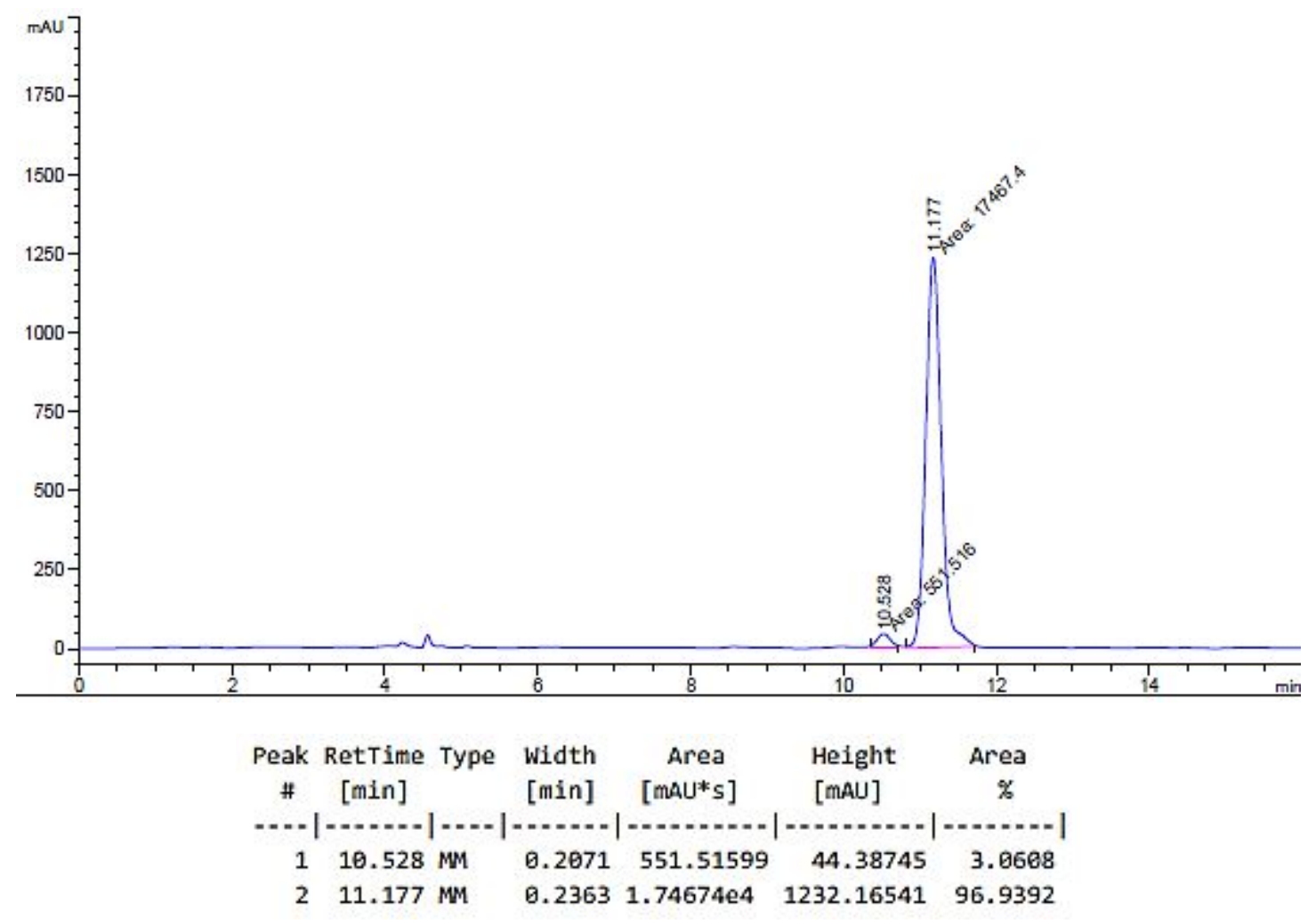

(S,E)-1-(benzyloxy)-9-chloro-2,2-difluoro-4-(p-tolyl)non-3-en-5-ol (28d) - ${ }^{1} \mathrm{H}$ NMR

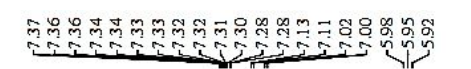

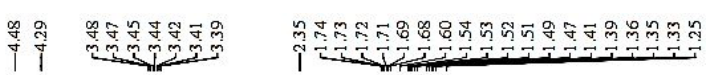

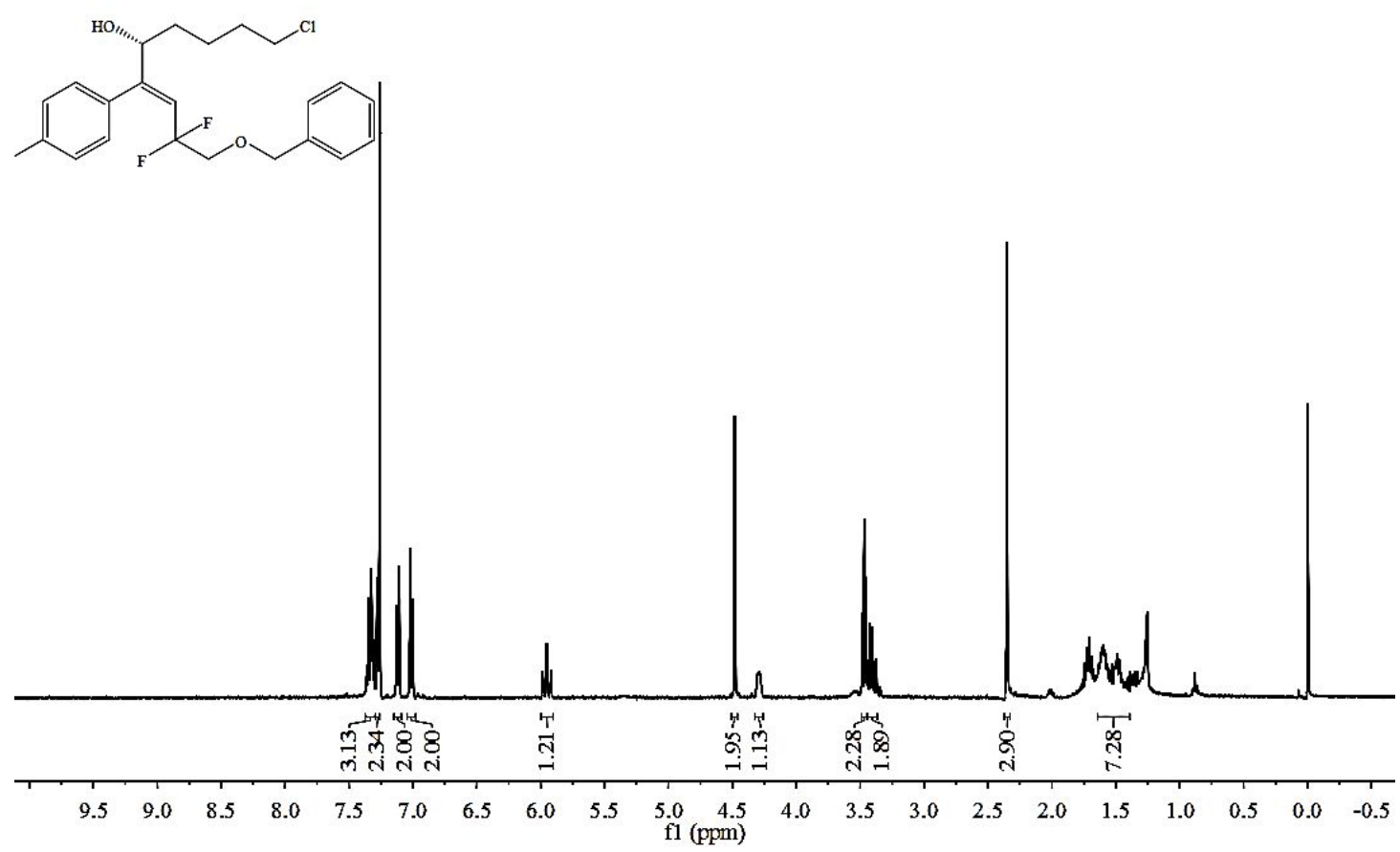

(S,E)-1-(benzyloxy)-9-chloro-2,2-difluoro-4-(p-tolyl)non-3-en-5-ol (28d) - ${ }^{13}$ C NMR 


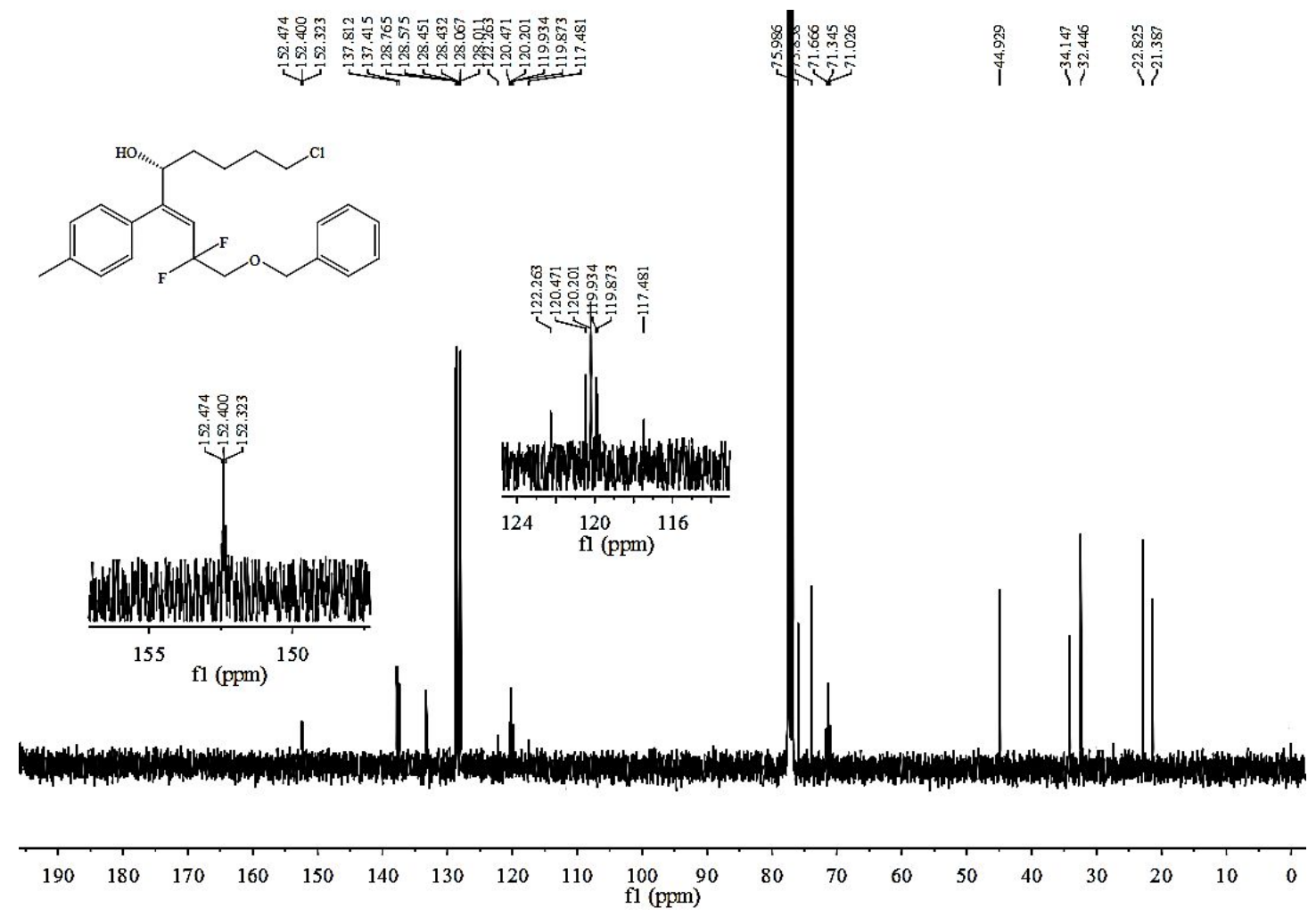

(S,E)-1-(benzyloxy)-9-chloro-2,2-difluoro-4-(p-tolyl)non-3-en-5-ol (28d) - ${ }^{19}$ F NMR

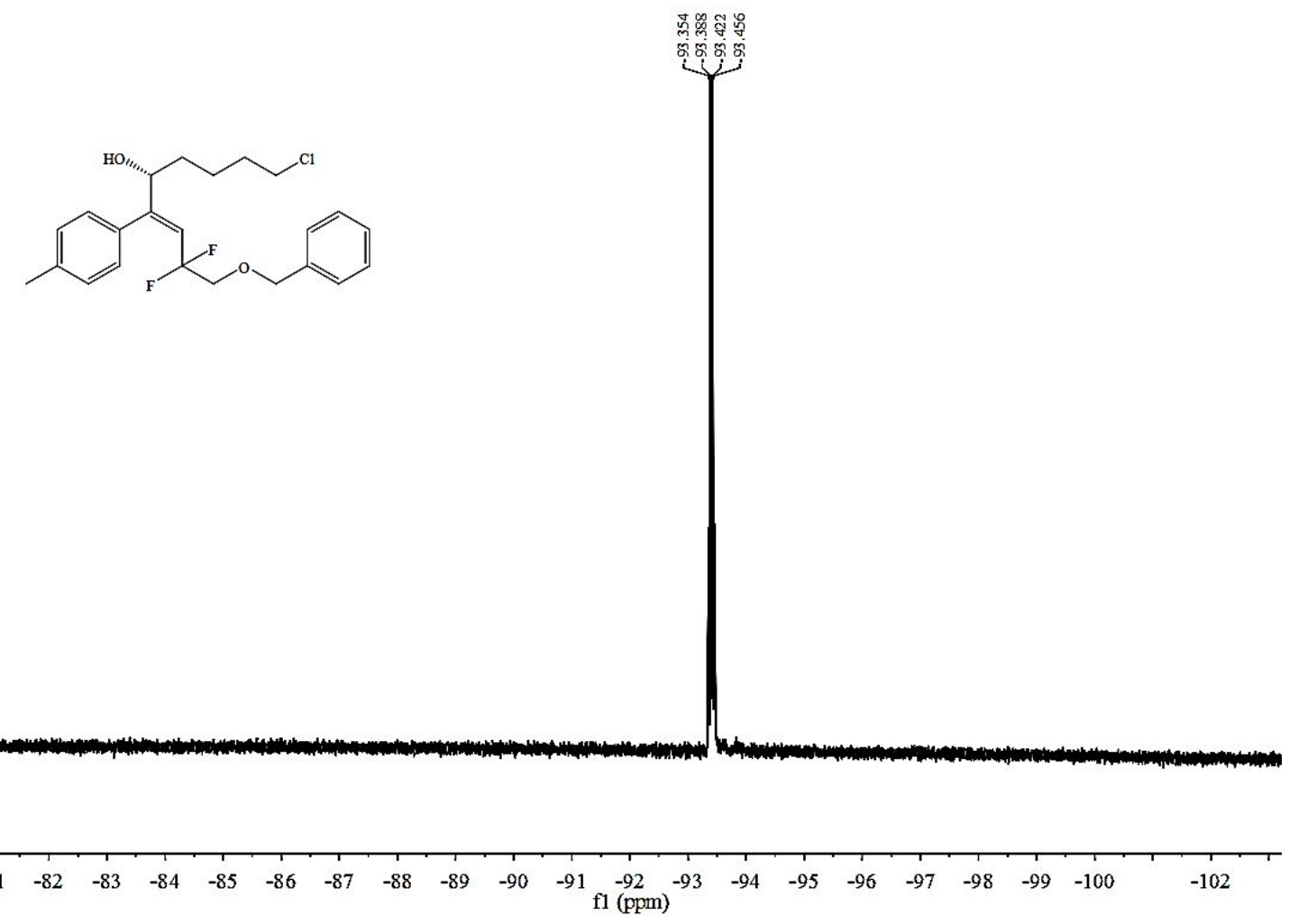

28d-HPLC (racemic) 


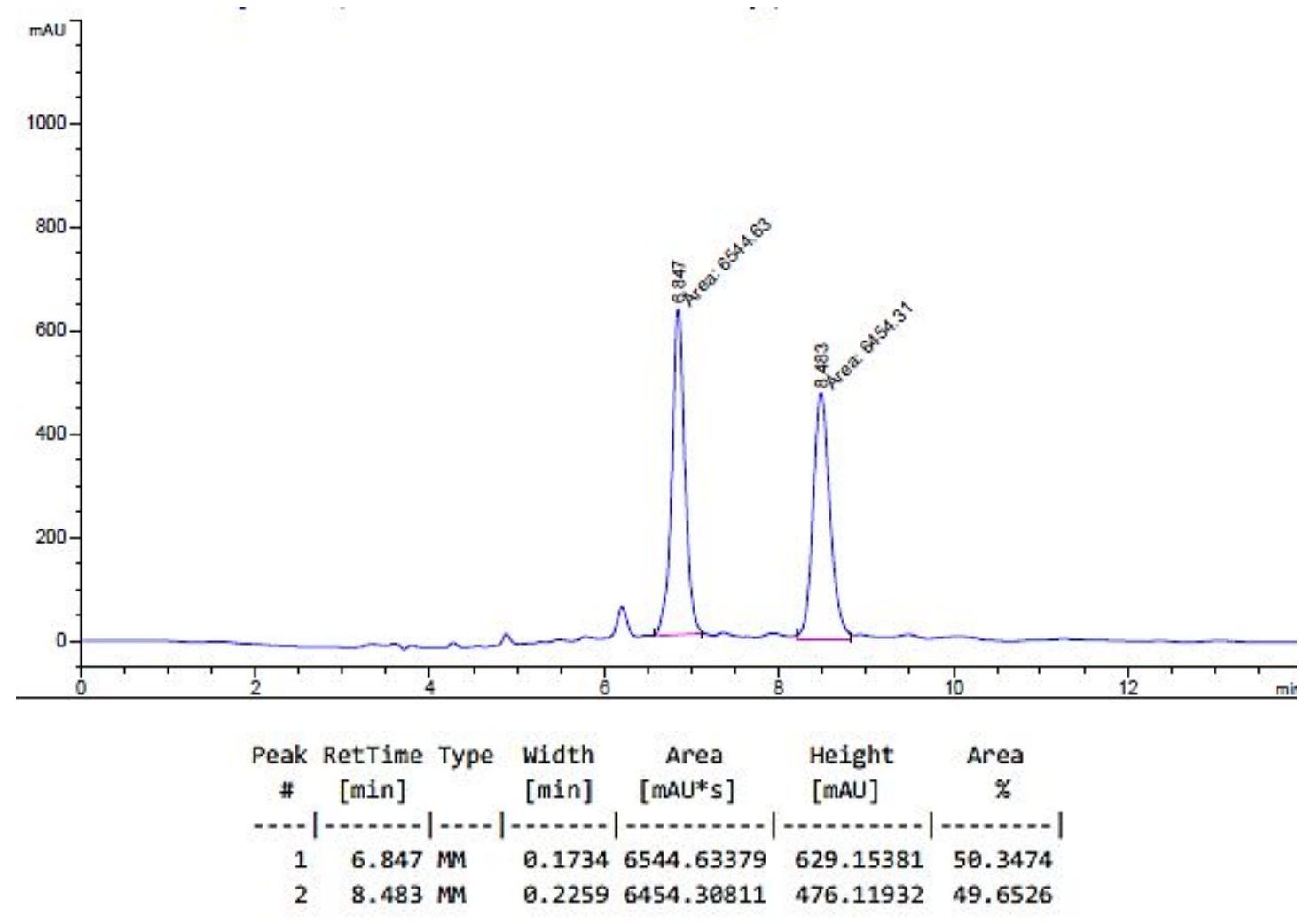

28d-HPLC (90\%)

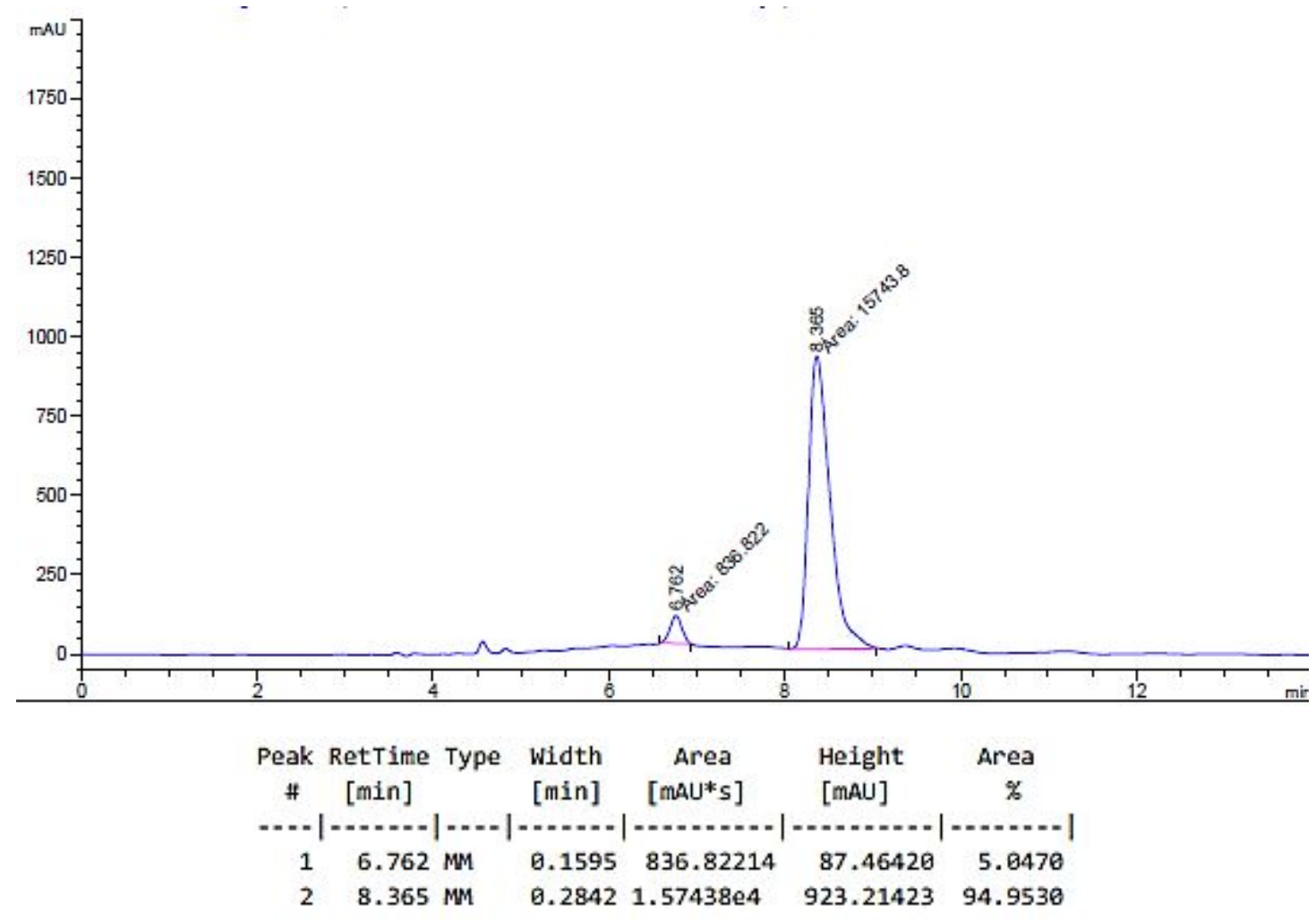

(S,E)-7,7,7-trifluoro-1,4-diphenylhept-4-en-3-ol (29d) - ${ }^{1} \mathrm{H}$ NMR 


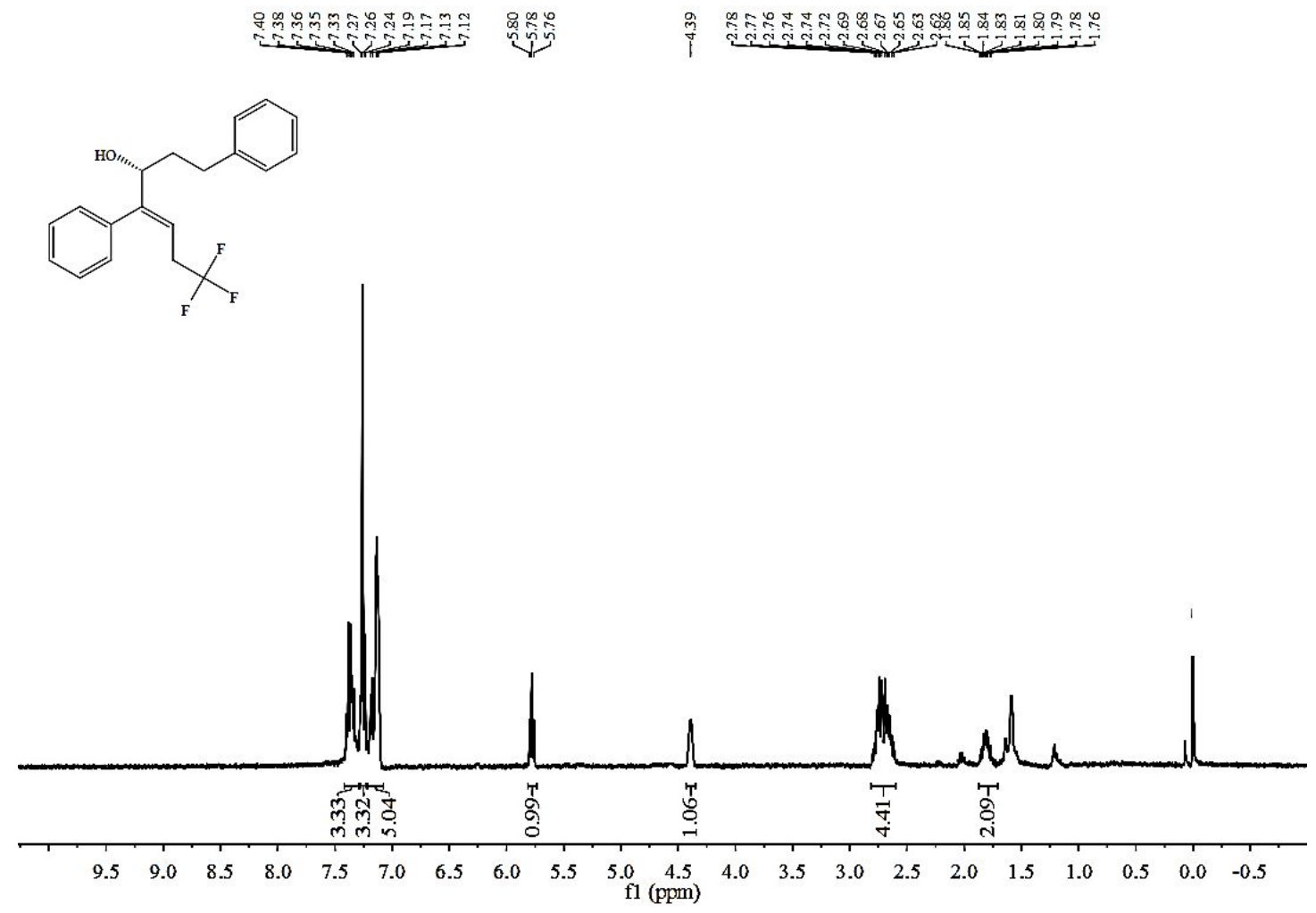

(S,E)-7,7,7-trifluoro-1,4-diphenylhept-4-en-3-ol (29d) - ${ }^{13} \mathrm{C}$ NMR

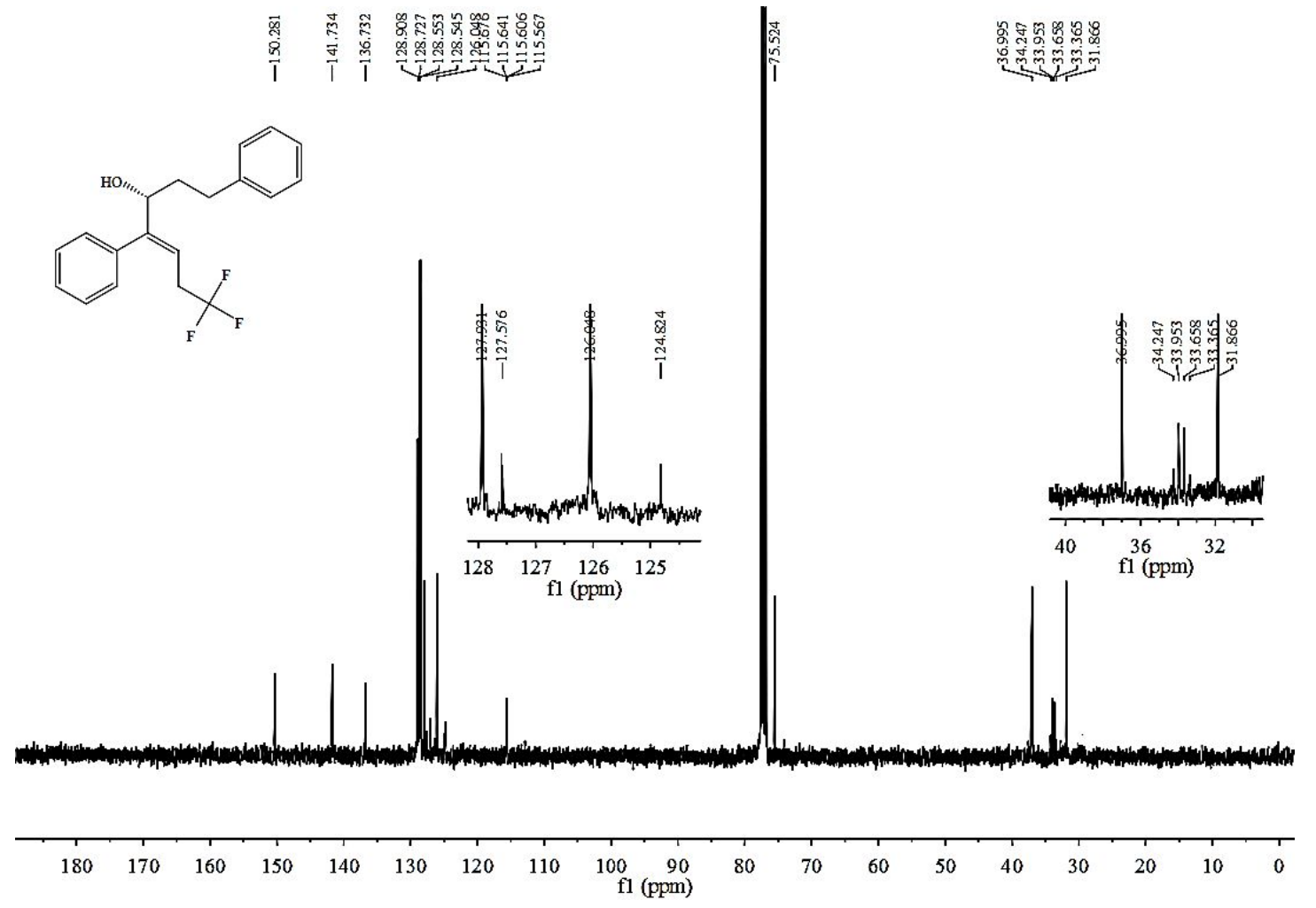

(S,E)-7,7,7-trifluoro-1,4-diphenylhept-4-en-3-ol (29d) - ${ }^{19}$ F NMR 


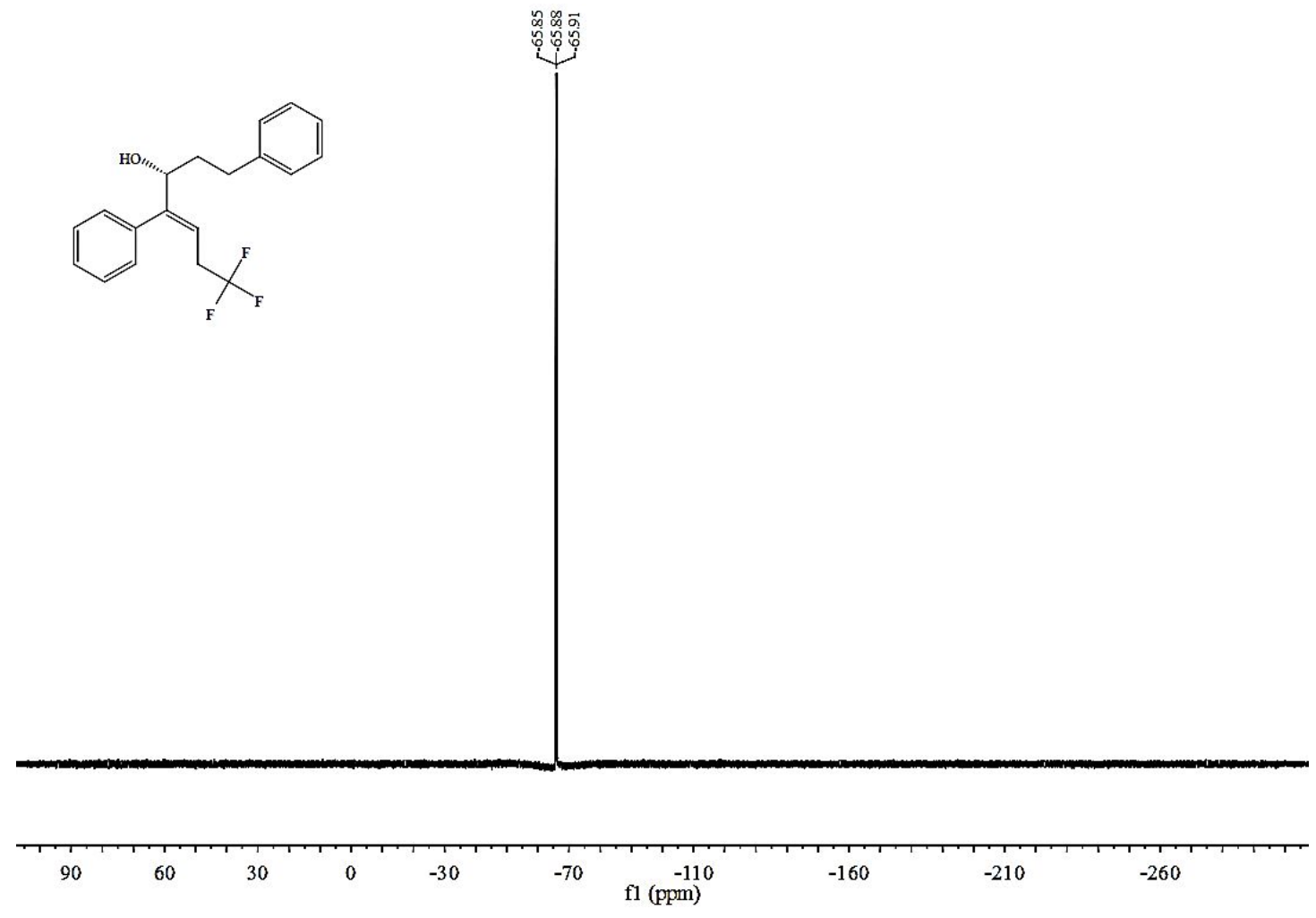

29d-HPLC (racemic)

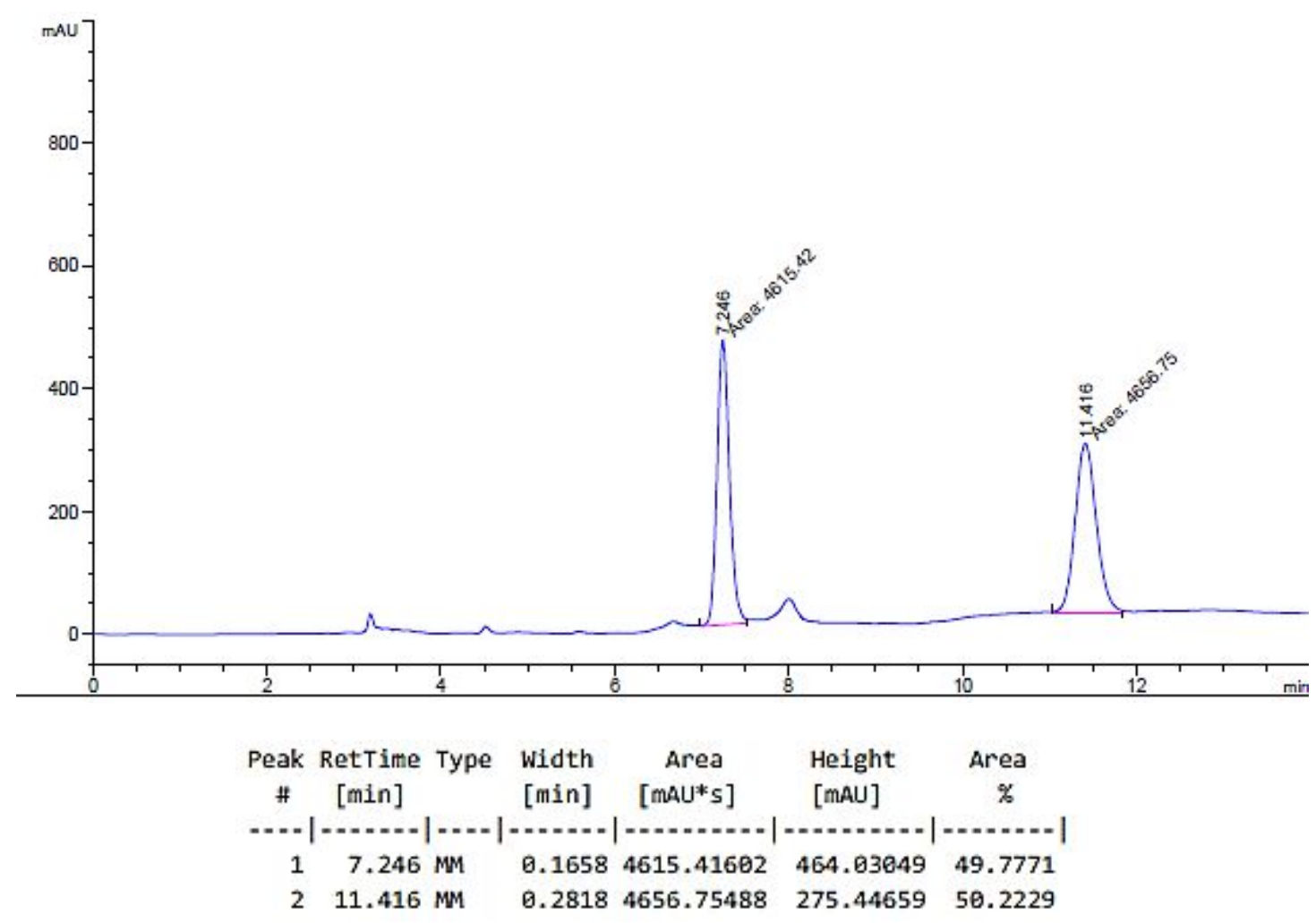

29d-HPLC (92\%) 

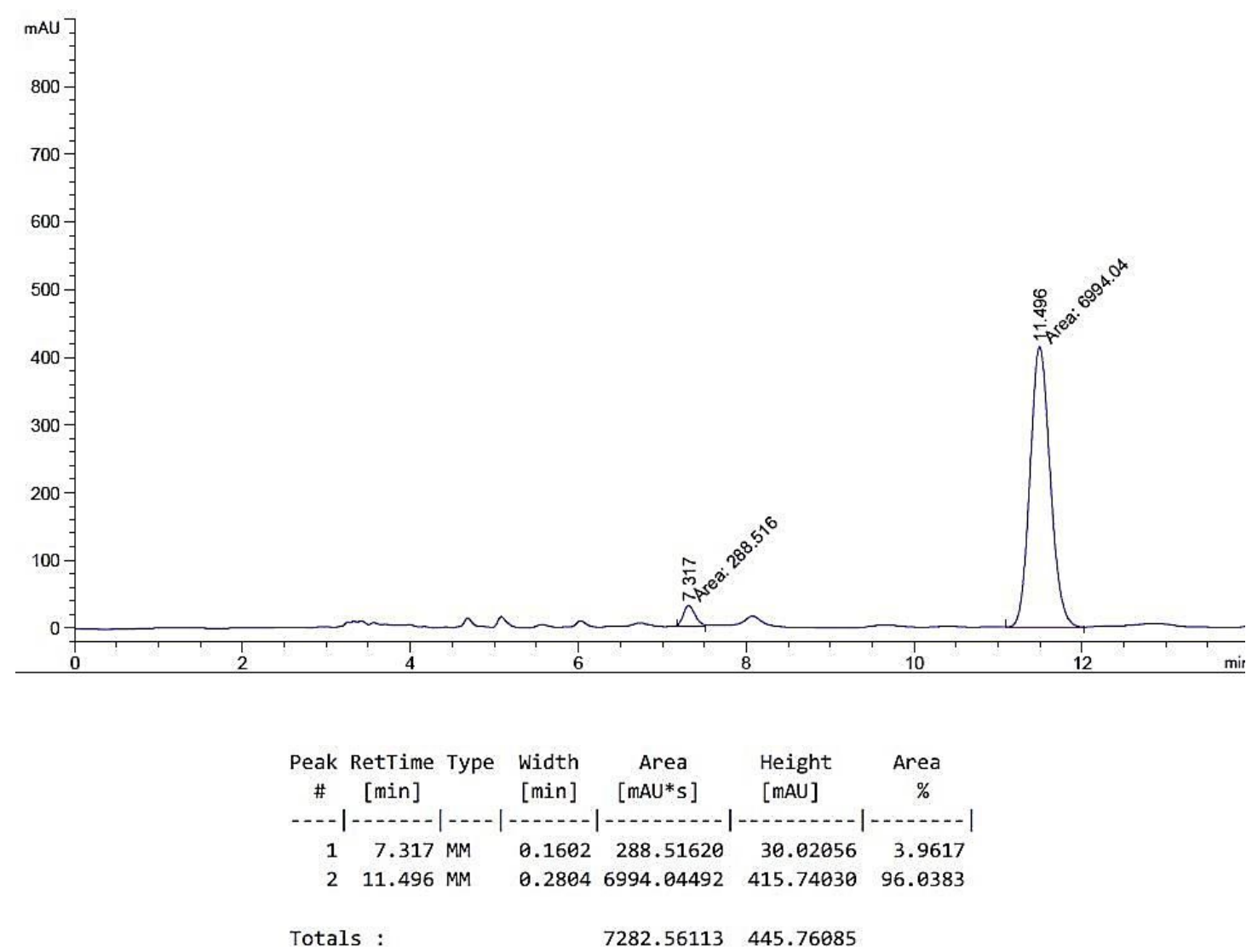

(S,Z)-5,5,5-trifluoro-1-phenyl-2-(thiophen-2-yl)pent-2-en-1-ol (30d) - ${ }^{1} \mathrm{H}$ NMR

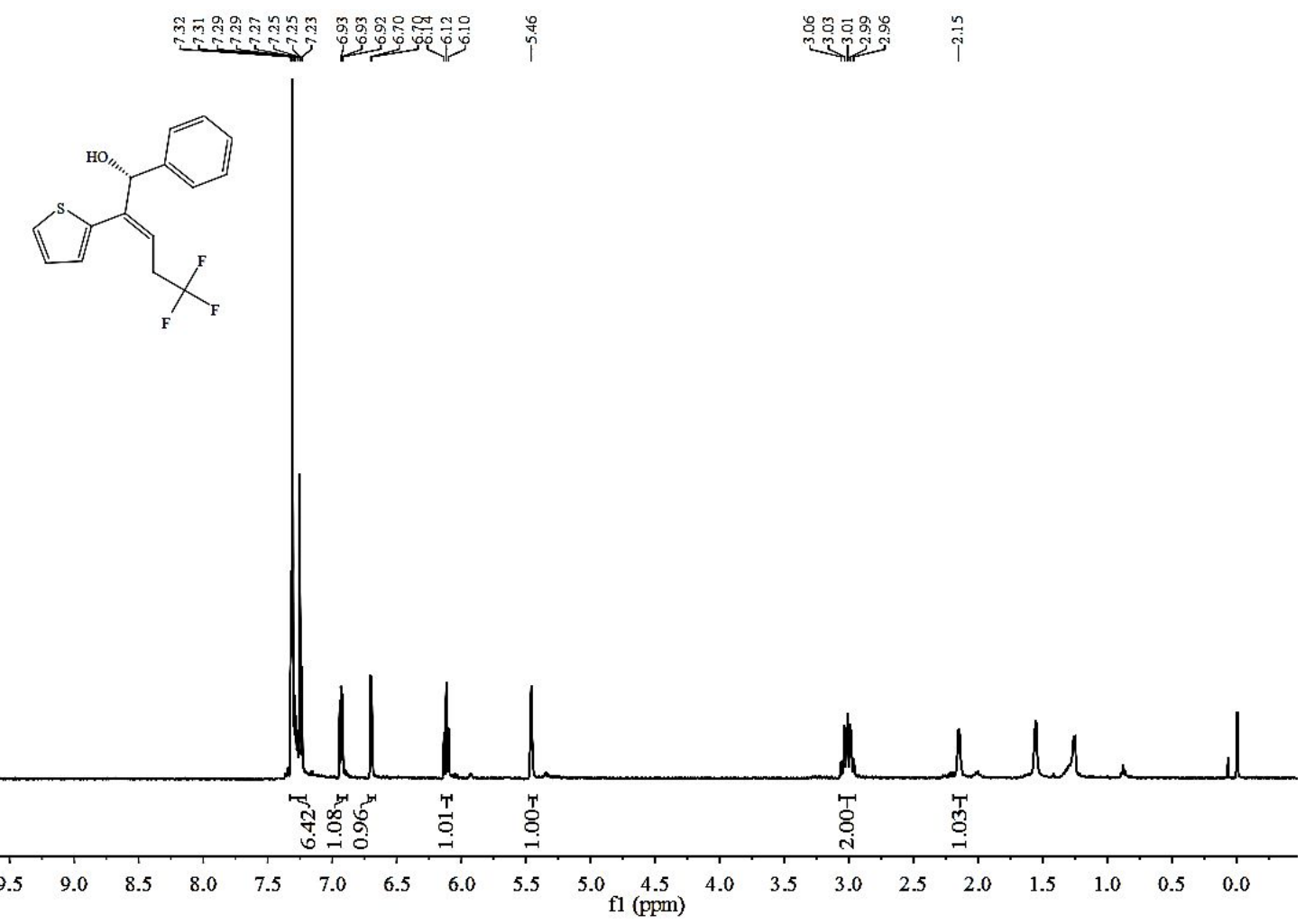

(S,Z)-5,5,5-trifluoro-1-phenyl-2-(thiophen-2-yl)pent-2-en-1-ol (30d) - ${ }^{13} \mathrm{C}$ NMR 


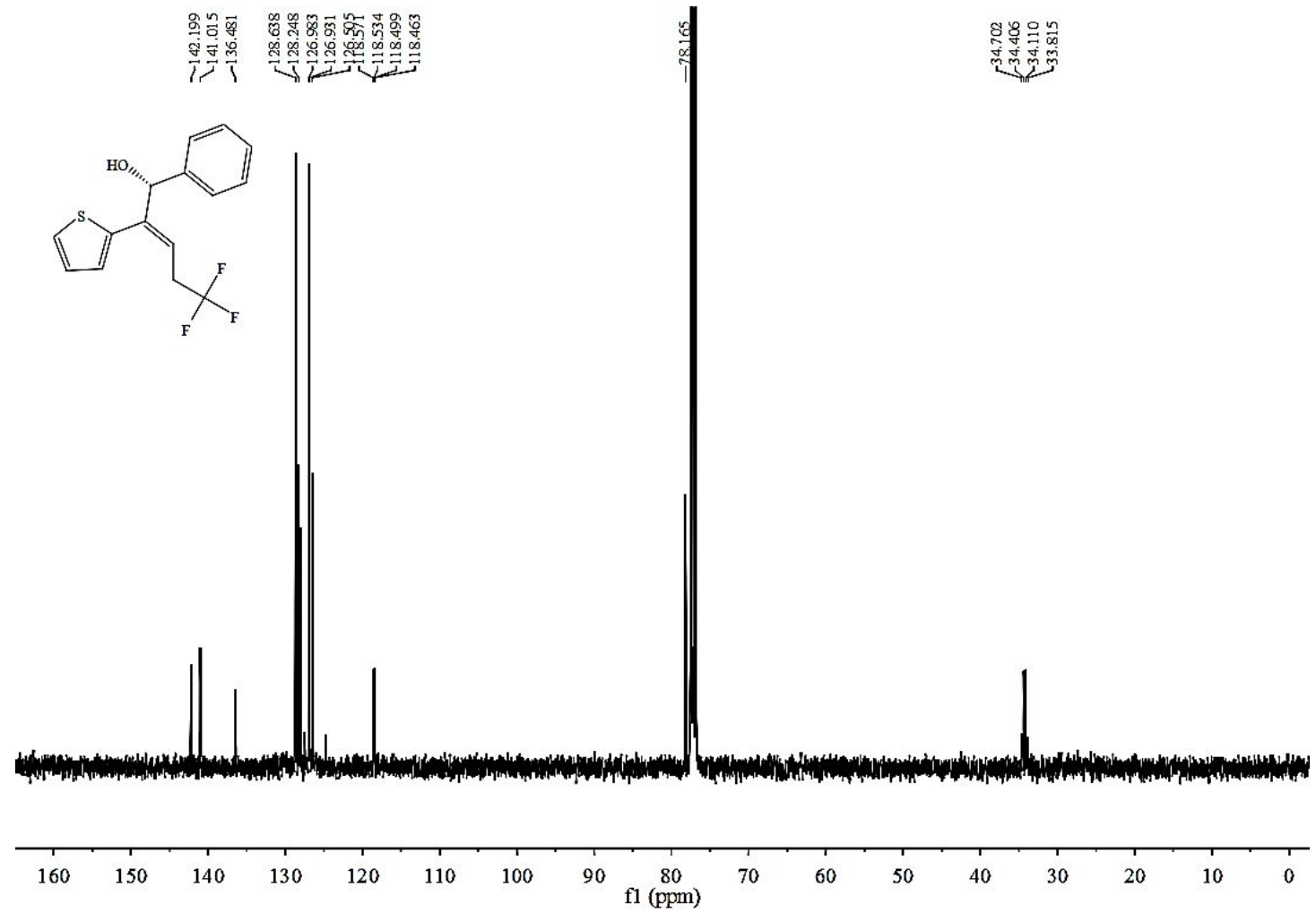

(S,Z)-5,5,5-trifluoro-1-phenyl-2-(thiophen-2-yl)pent-2-en-1-ol (30d) - ${ }^{19}$ F NMR
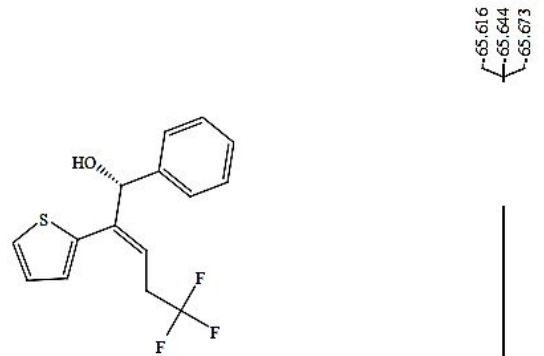

i

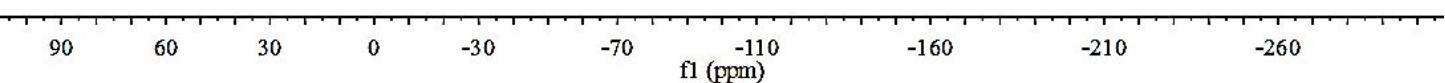

30d-HPLC (racemic) 

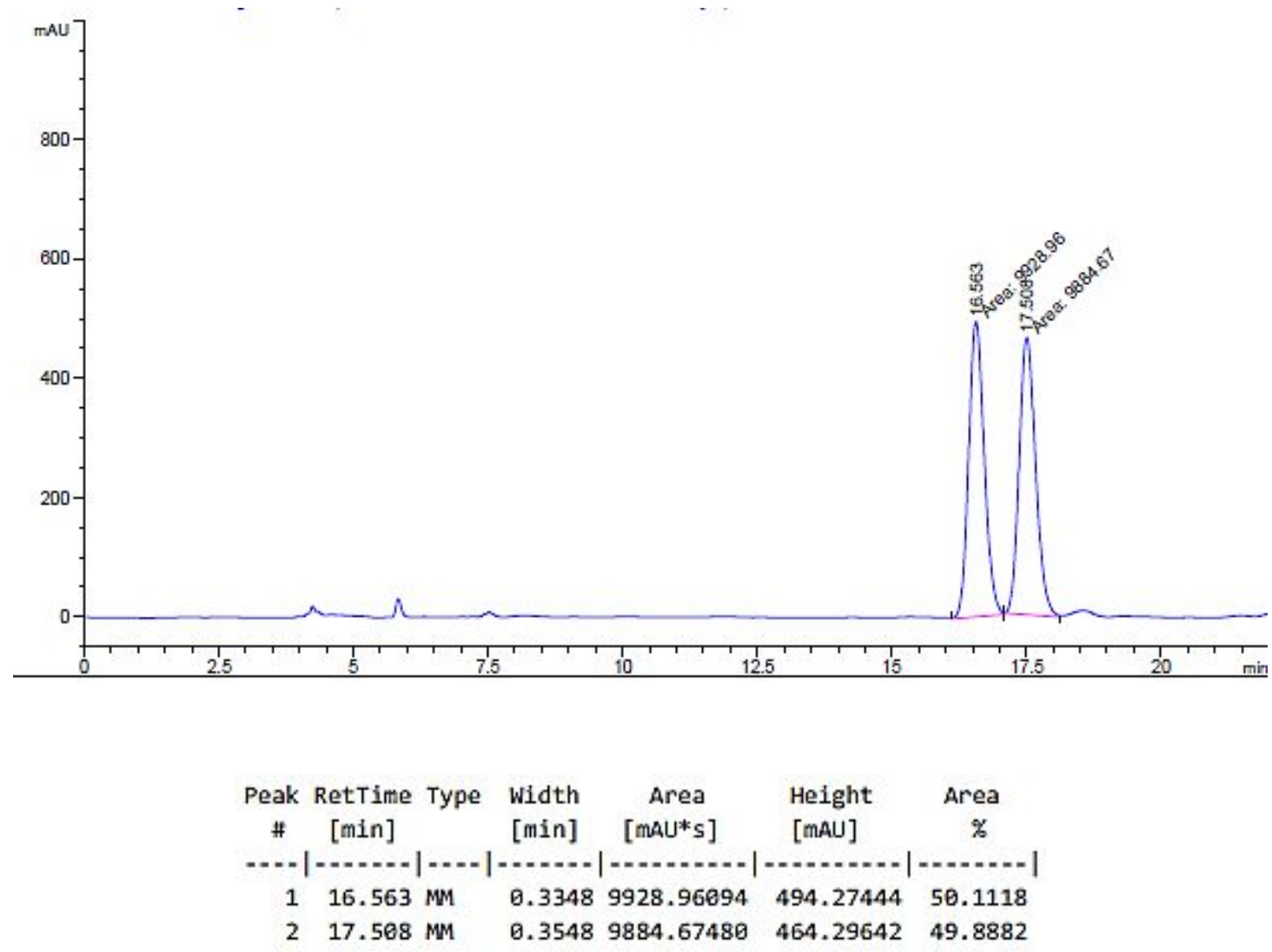

30d-HPLC (racemic)

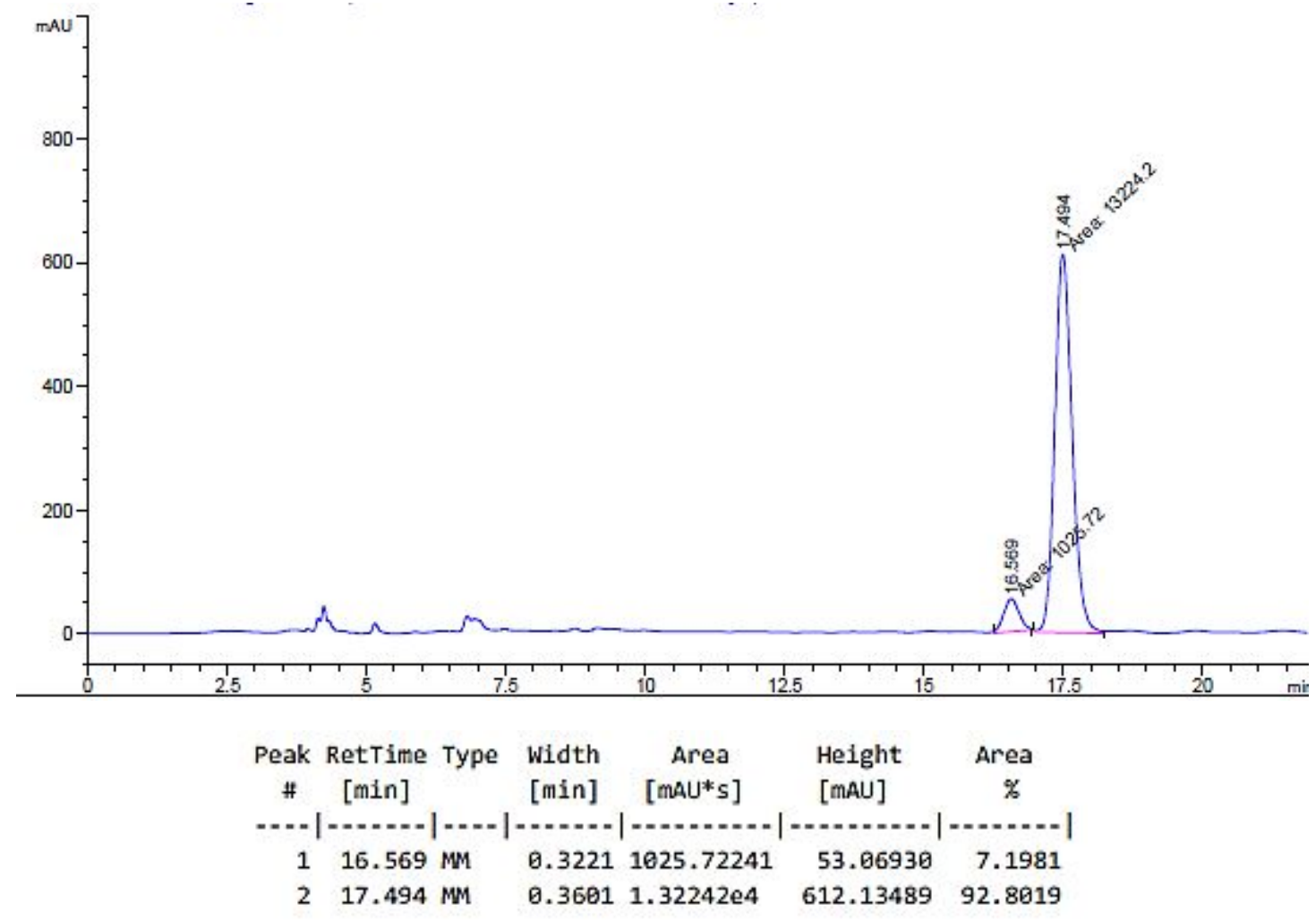

(2). Copies of NOESY Spectra 
NOESY NMR (S,E)-2,2-dimethyl-4-phenyldec-3-en-5-ol (3d)

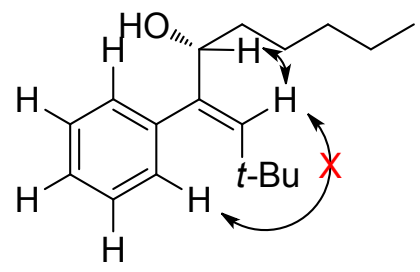

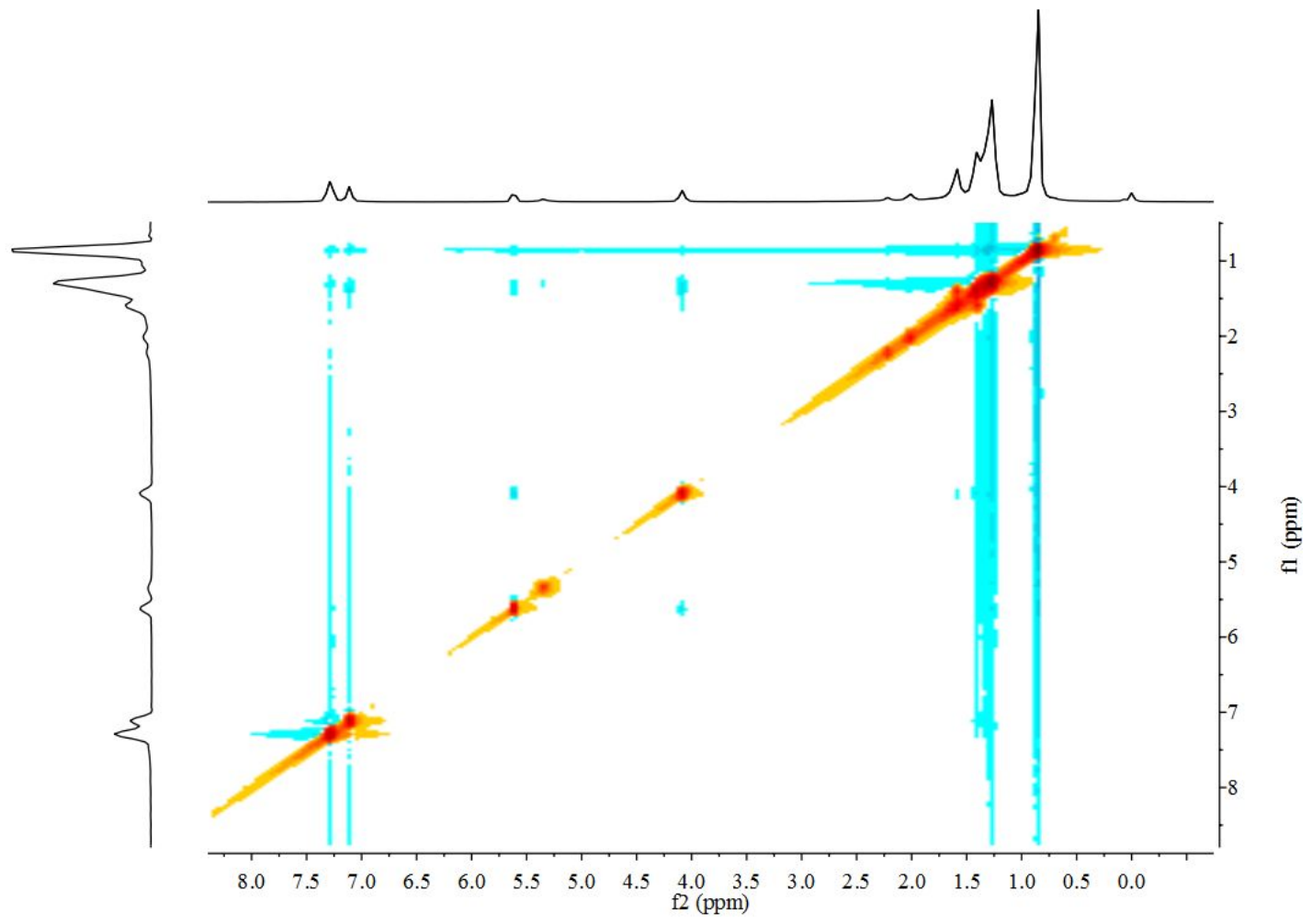

